\title{
Macrophages in colorectal cancer: proof of principle for diagnostic application
}

Citation for published version (APA):

Japink, D. (2015). Macrophages in colorectal cancer: proof of principle for diagnostic application. [Doctoral Thesis, Maastricht University]. Maastricht University. https://doi.org/10.26481/dis.20150424dj

Document status and date:

Published: 01/01/2015

DOI:

10.26481/dis.20150424dj

Document Version:

Publisher's PDF, also known as Version of record

\section{Please check the document version of this publication:}

- A submitted manuscript is the version of the article upon submission and before peer-review. There can be important differences between the submitted version and the official published version of record.

People interested in the research are advised to contact the author for the final version of the publication, or visit the DOI to the publisher's website.

- The final author version and the galley proof are versions of the publication after peer review.

- The final published version features the final layout of the paper including the volume, issue and page numbers.

Link to publication

\footnotetext{
General rights rights.

- You may freely distribute the URL identifying the publication in the public portal. please follow below link for the End User Agreement:

www.umlib.nl/taverne-license

Take down policy

If you believe that this document breaches copyright please contact us at:

repository@maastrichtuniversity.nl

providing details and we will investigate your claim.
}

Copyright and moral rights for the publications made accessible in the public portal are retained by the authors and/or other copyright owners and it is a condition of accessing publications that users recognise and abide by the legal requirements associated with these

- Users may download and print one copy of any publication from the public portal for the purpose of private study or research.

- You may not further distribute the material or use it for any profit-making activity or commercial gain

If the publication is distributed under the terms of Article $25 \mathrm{fa}$ of the Dutch Copyright Act, indicated by the "Taverne" license above, 
Macrophages in Colorectal Cancer:

proof of principle for diagnostic application 
Copyright (c) 2015 by Dennis Japink. All rights reserved.

ISBN/EAN: 978-94-6182-551-3

Cover illustration: Dennis Japink

Thesis illustrations: Simon Desarzens (simondesarzens.blogspot.com)

Thesis layout: Tiny Wouters and Dennis Japink

Thesis photography: Joëlle Suijkerbuijk en Dennis Japink

Printed by: offpage.nl

This author of this thesis has been funded by ZonMw by means of an AGIKO-grant. Furthermore funding was provided by obtaining the Maastro-Atrium grant assigned to the author by Atrium MC Parkstad, Heerlen and the MAASTRO Clinic, Maastricht. Finally the Maatschap Heelkunde Zuid-Limburg (MHZL) of the Atrium MC Parkstad, Heerlen and the Maatschap Algemene Heelkunde of the MUMC Maastricht have been kindly funding the author for a total of three years.

Reproduction of this thesis was kindly and unconditionally supported by gifts of (in alphabetical order):

Atrium Medisch Centrum Parkstad, Beckton \& Dickinson, Boehringer Ingelheim, ChipSoft, Covidien, Maastricht University Medical Center (MUMC ${ }^{+}$), Maatschap Heelkunde ZuidLimburg (MHZL), Nutricia, Pfizer, Terumo, and Uludag Surgery, which is gratefully acknowledged.

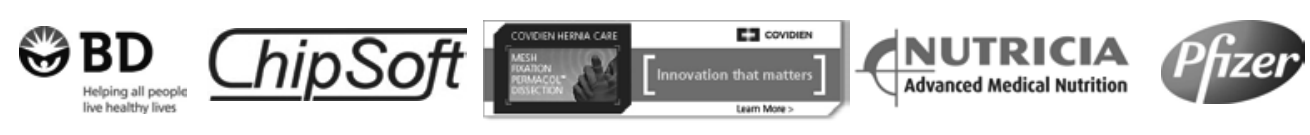




\title{
Macrophages in Colorectal Cancer:
}

\author{
proof of principle for diagnostic application
}

\author{
PROEFSCHRIFT \\ ter verkrijging van de graad van doctor aan de Universiteit Maastricht, \\ op gezag van de Rector Magnificus, Prof dr. L.L.G. Soete, \\ volgens het besluit van het College van Decanen, \\ in het openbaar te verdedigen \\ op vrijdag 24 april 2015 om 10.00 uur
}

door

Dennis Japink

geboren op 20 september 1978

te Ermelo 
Promotor

Prof. dr. M.F. von Meyenfeldt

\section{Copromotores}

dr. ing. M.P.G. Leers, Atrium MC Parkstad, Heerlen

dr. M.N. Sosef, Atrium MC Parkstad, Heerlen

dr. M. Nap, Atrium MC Parkstad, Heerlen

Beoordelingscommissie

Prof. dr. L.P.S. Stassen, voorzitter

Prof. dr. ir. E. Biessen

Prof. dr. A. Masclee

Prof. dr. F. Tacke, Universitätsklinikum Aachen, Duitsland

Prof. dr. L. Ziegler-Heitbrock, Helmholtz Zentrum München, Duitsland 


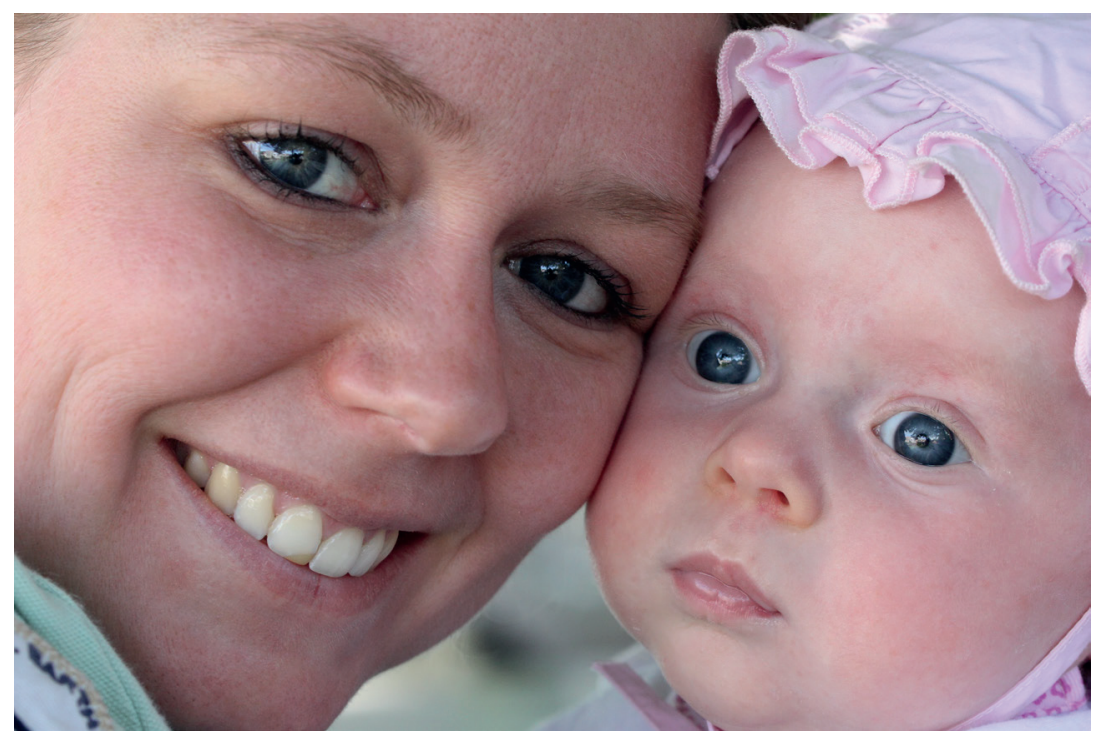

voor mijn meisjes... 



\section{Contents}

Chapter 1 General introduction (review):

Macrophages in colorectal cancer: current diagnostic opportunities $\quad 9$

Chapter 2 Activated macrophages containing tumor marker in

colon carcinoma: immunohistochemical proof of a concept

Chapter 3 Visualising CEA in activated macrophages using electron

microscopy

Chapter $4 \quad$ CEA in activated macrophages. New diagnostic possibilities for tumor markers in early colorectal cancer

Chapter 5 Reproducibility studies for experimental epitope detection in macrophages (EDIM)

Chapter 6 Predicting response using blood biomarkers and PET-CT

Summary

Samenvatting

Valorisation

Dankwoord

Curriculum Vitae

List of publications

Abbreviations 



\section{CHAPTER 1}

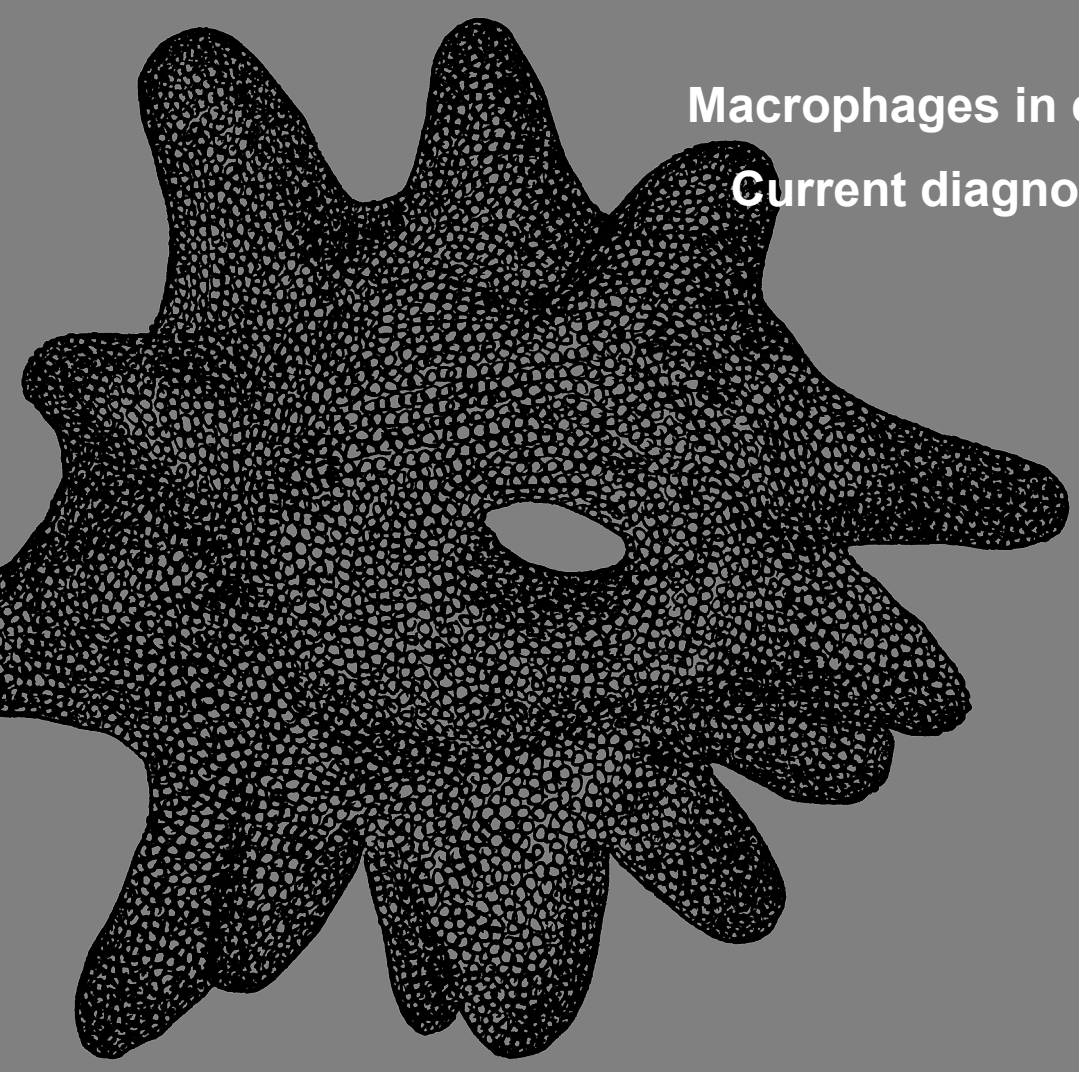

Dennis Japink Maarten F von Meyenfeldt Marius Nap Meindert N Sosef Geerard L Beets Math PG Leers Excerpts have been submitted 


\section{Abstract}

Current cancer diagnostic trajectories consist of clinical evaluation, various imaging techniques, histological assessments, biomarker assays and, if applicable, endoscopic investigations. Biomarker assays are known to focus on serum tumour markers, such as tumour proteins or tumour DNA. In recent literature a focus has emerged on the role of the immune system in cancer origin and development. In the immune system of healthy persons monocytes and macrophages play an important role in managing tissue homeostasis by patrolling, cytokine production and antigen presenting capabilities towards other immune cells. They form the first barrier of defence in the intestinal tract and are able to clear invasive pathogens. In patients with colorectal cancer (CRC) macrophages play an important role in tumour progression due to their plasticity. Subpopulations are formed with their own specific tasks based on tumour cell influences. Methods to diagnose or monitor CRC utilising the immune cell populations are new and challenging. CRC is the fourth most common form of cancer in the Netherlands after prostate cancer, breast cancer and melanoma. Key in diagnosis and treatment is the ability to detect primary and recurrent disease in time for curation. In this review the origin and role of macrophages in cancer is described, with a focus on the non-hereditary form of CRC. The available screening, diagnostic and monitoring methods for CRC are described to provide context for experimental diagnostic and monitoring methods including macrophage-assays. 


\section{Macrophages in (colorectal) cancer}

\subsection{The monocyte phagocyte system}

Monocytes, macrophages and dendritic cells (DCs) are part of the mononuclear phagocyte system. Their progenitors (such as haematopoietic stem cells (HSC) and common myeloid progenitors (CMP)) have their origin in bone marrow ${ }^{1}$. Macrophages and DCs develop from monocytes as their direct precursor, both having their own specific functions within the immune system. Originally identified by the Russian immunologist Metchnikoff during the 1880's, macrophages (called phagocytes at that time) are ancient phagocytic cells, being part of the innate immune system. "Macrophage" literally means "large eating cell", one of its prime functions and a description of its morphology ${ }^{2}$ Macrophages are designated self and non-self identifiers. This enables them to not only discern host from foreign substances, but also to recognise host elements that have become "foreign", such as damaged or dying cells ${ }^{2}$. These dying cells can be part of stromal tissue consisting of tumour and immune cells ${ }^{3}$.

\subsection{Monocyte and macrophage activation}

Until a few years ago, two distinct pathways for macrophage activation were described in literature, the classic (M1) and alternative (M2) activation ${ }^{4,5}$. In cancer, activation status was thought to depend on the stage of tumour development ${ }^{6,7}$. M1-macrophages were considered pro-inflammatory, activated to react on infiltration or tissue damage by non-self entities. Sometimes M1-type reactions would be excessive, and therefore lead to prolonged inflammation. This results in (chronic) tissue-damage as found in diseases such as Crohn's disease or ulcerative colitis. The widespread inflammation in turn would lead to extensive DNA-repair and inherent mutations, prone to become carcinogenic. The M2-macrophages were found to exhibit an immunosuppressive profile (T-cell inhibition and tumour growth promotion ${ }^{8}$ ). This has been proven present in human tumours such as ovarian carcinoma and glioma ${ }^{9,10}$. Recent literature describes more of an activation continuum where M1 and M2 are each the extremes. This continuum consists of macrophages flexibly categorised in classically activated, wound-healing and regulatory macrophages, dependent on their cell surface marker expression, cell function/secretions and signalling specifications ${ }^{11}$. Macrophage plasticity and diversity is currently investigated in 
relation to inflammatory (colorectal) diseases and their proposed subsequent malignant evolution. It is thought that when monocytes are recruited locally they must undergo a rapid local education process to support homeostasis. Otherwise alternative fates are adopted, possibly promoting inflammation ${ }^{12}$.

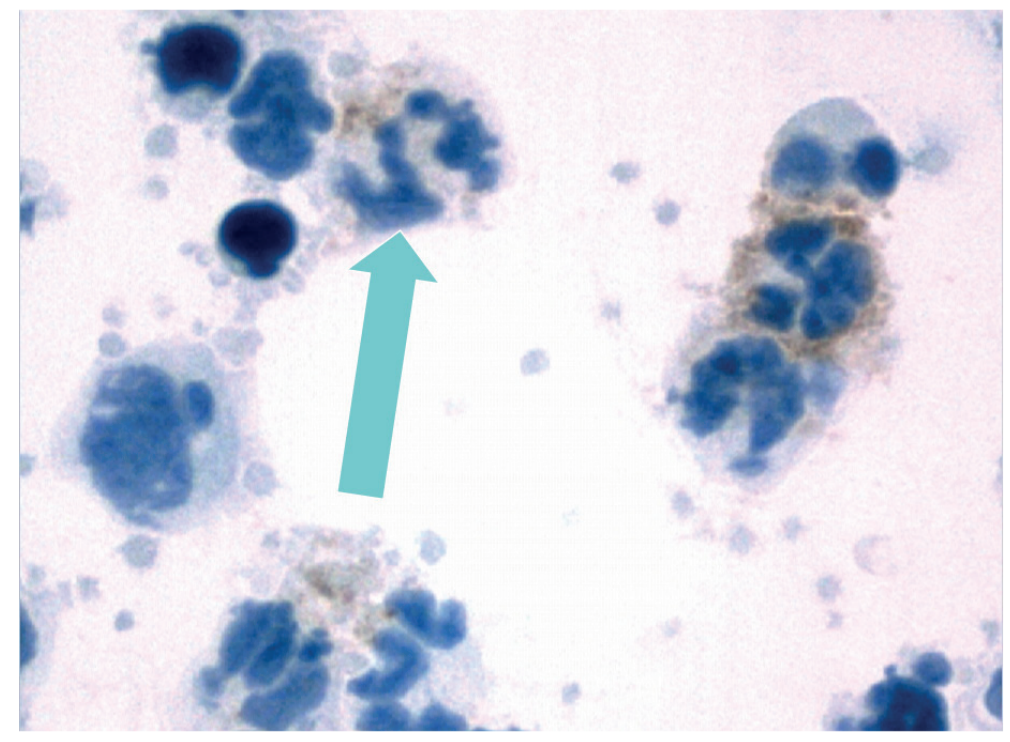

Figure 1.1 Macrophage with bluish core staining, showing a brownish prostate specific antigen (PSA) staining intracellularly (Leers et al 2008). The large irregular cell with multilobulated core shows the distinct morphology of the macrophage.

\subsection{Monocyte heterogeneity; three distinct subtypes}

In humans, heterogeneity of monocytes can be immunophenotypical shown by the expression of CD14 (LPS-binding protein complex) and CD16 (Fcy-receptor type III). CD16 is a marker of monocyte activation. The simultaneous expression of CD14 with CD16 enables detection of differentiated activated monocytes resembling circulating and tissue macrophages ${ }^{13-15}$. It is known that CD16+ monocyte levels are elevated in metastatic gastric, colon and rectal cancer patients as well as in sepsis patients ${ }^{16}$. CD $16+$ monocytes account for $46 \% \pm 22 \%$ in cancer patients versus $5 \% \pm 3 \%$ for controls. It has been proposed that the 
CD16+ population would offer a readily accessible, quantifiable means by which to identify and follow a host's response to treatment ${ }^{17}$. The majority of monocytes are CD14++CD16- (classic monocytes, $90 \%$ of the total monocyte population). The CD16+ monocytes can be subdivided into CD14+C16++ (nonclassical) monocytes and CD14++CD16+ (intermediate) monocytes. The latter mentioned subset (including atypical macrophages and dendritic cells) ${ }^{18}$ has an intermediate phenotype between the classical and non-classical monocytes with a higher antigen presenting activity and pro-angiogenic capacity (Figure 1.2) ${ }^{19,20}$. These intermediate cells show CD163 positivity concurrent with the classical monocytes, which differentiates them from the non-classical subtype. A low level of CD62L (L-selectin) is observed in non-classical monocytes combined with a low level of chemokine receptor 2 (CCR2), which is not seen in the classical and intermediate subtypes. This last finding has also been reproduced in mice, rats and pigs, suggesting a homology across species ${ }^{19}$.

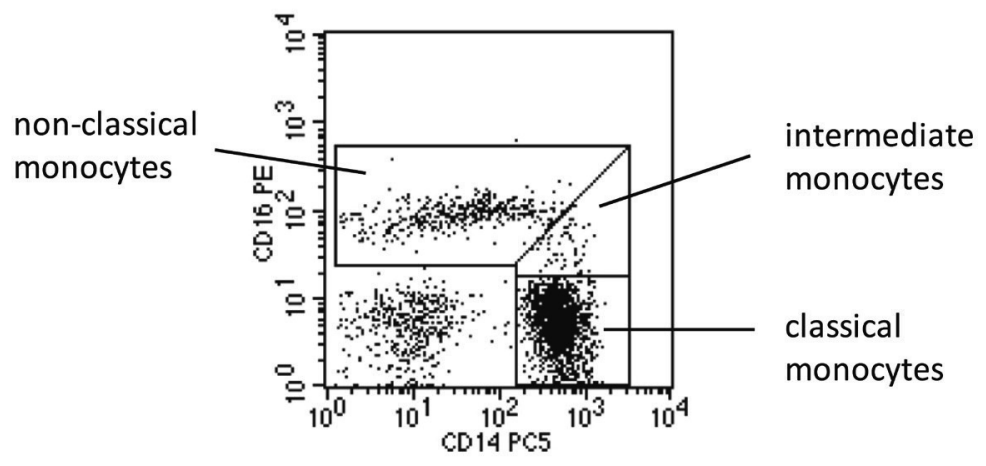

Figure 1.2 Example of monocyte subsets gating strategy in humans as defined by flow cytometry (Ziegler-Heitbrock, 2014)

Ziegler-Heitbrock et al. described in 1993 that CD14+CD16+ monocytes exhibit a higher level of class II antigen and a lower level of CD11b and CD33 antigens compared to the CD14++ monocytes. The CD14+CD16+ monocytes are therefore known as a more mature monocyte type. The consistency with tissue macrophages has been investigated by stimulation of peripheral blood mononuclear cells. This resulted in a higher class II antigen and lower CD11b/CD33 antigen expression confirming acquisition of features in common with mature tissue macrophages ${ }^{13}$. 
In mice, macrophage cell surface expressions of CD11c and CD11b in combination with $\mathrm{F} 4 / 80$ antigen are characteristic for monocyte-derived macrophages residing in the lamina propria of the colon. Besides that, they express $\mathrm{CD} 64$ and $\mathrm{CX}_{3} \mathrm{CR} 1^{12}$. The latter enables (flow cytometrical) macrophage detection in mice-experiments using GFP-reporter gene insertion ${ }^{21}$.

\subsection{Monocyte and macrophage trafficking}

Investigation of macrophages using a murine model enables visualisation of macrophage movement, also known as trafficking. By means of GFP-labelling, trafficking has been shown in vivo in mice ${ }^{22,23}$. Patrolling monocytes were found to roll over the intraluminal vessel wall and subsequently extravasate. Monocytes, which extravasated into the gut, are shown to be able to retain their CD14/CD16 monocyte state and migrate further after internalising substances in the tissue. Migration towards lymph nodes has been reported ${ }^{24}$. In other situations, such as in atherosclerosis ${ }^{25}$ and clearance of photoreceptor debris following retinal injury ${ }^{26,27}$, it has been shown that macrophages are able to migrate out of the tissue they resided in, back into the blood stream. A postulated hypothesis is that monocytes indeed extravasate, possibly differentiate into macrophages and perform local phagocytosis at the edges of (invasive) tumour growth. Subsequent migration back into the blood stream or to lymph nodes and beyond has been investigated ${ }^{28,29}$ and was confirmed by our research group ${ }^{30}$.

\subsection{The origin of the tissue-macrophage}

In combination with other mononuclear phagocytes, macrophages maintain tissue integrity during the development, initiation and resolution of innate and adaptive immunity. The continuity of the population of macrophages was historically thought to be mainly dependent on replenishment out of the blood stream by differentiating activated monocytes. Recently, organ-resident macrophages were discovered to derive from fetal progenitors developing out of the yolk sac during embryogenesis in specific organs ${ }^{31}$. Under steady state they exhibit the capacity for local self-maintenance without significant input of circulating monocytes, as was found for liver Kupffer cells (KC), lung alveolar macrophages, peritoneal macrophages, epidermal Langerhans cells and brain microglia $^{32-36}$. On the other hand, experiments on mouse-gut showed that macrophages were found to repopulate mainly out of HSC-derived (activated) Ly6 $\mathrm{C}^{+}$-monocytes. After extravasation from the blood stream they complement 
the resident macrophage population. As a result, this highly versatile macrophage compartment of the total immune cell population in the lamina propria is able to rapidly adapt to the unique and dynamic intestinal environment it encounters ${ }^{12}$.

\subsection{Tumour-associated macrophages (TAM)}

Macrophages present in malignant tumours are called tumour-associated macrophages (TAM) ${ }^{8}$. TAM, apart from other myeloid cells including neutrophils, eosinophils and dendritic cells, can represent up to $50 \%$ of the total tumour mass. This infiltration grade was thought to be an indication of anti-tumour immunity, by now it is clear that this function is suppressed in the tumour environment. Macrophage-mediated cancer cell phagocytosis can be reinvigorated by a therapeutic intervention blocking CD47, a "don't eat me" signal over-expressed in most cancer cells ${ }^{8,37}$. In breast, gastric, urogenital, glioma, cholangiocarcinoma, Hodgkin lymphoma and glioma high density TAM infiltration is associated with worse overall survival, enhanced tumour growth and metastases. Interestingly though, in CRC high density TAM infiltration is associated with better overall survival ${ }^{38}$. It is suggested that TAM ontogeny, phenotype, their products and activation state, are more informative for patient prognosis than their absolute number in and around tumours ${ }^{8,39}$

\subsection{TAM in stromal tissue}

The stromal border around CRC consists of different macrophage-phenotypes, among other cell types such as T-cells, fibroblasts, dendritic cells and infiltrating leukocytes. In literature, the tumour cell to immune cell interactions have been described as cancer immunoediting. This phenomenon has a much broader basis than macrophages alone $e^{40-42}$. This review will not further dwell on the origins, functions and interactions of the other mentioned cells in the stromal border or their role in cancer development in general ${ }^{40}$. Macrophages contribute to a tumour microenvironment suitable for either tumour progression or tumour regression, where the outcome is based on an environmental balance shift either way ${ }^{11,38}$. TAM were thought to predominantly lean towards a M2-like phenotype ${ }^{43-45}$, but recent research shows that a dynamic activation switch occurs among TAM as the tumour develops, shifting from M1-state through different sub-states towards M2. This finding may help explain the mixed activation state of TAM populations found in different murine and human tumours $^{44,46,47}$. In chronic unresolved inflammation sites, macrophages in $\mathrm{M} 1$ 
status are considered more carcinogenic due to their ability to maintain and aggravate the inflammatory reaction. M2 macrophages (low IL-12 expression, high IL-10 expression and low tumouricidal activity) show more tumour promoting capabilities. These are based on trophic and wound healing properties, which promote angiogenesis and tissue remodelling ${ }^{8,48}$. Mouse studies show tumour progression to be associated with phenotypical switches from M1 to M2 macrophages ${ }^{49}$. Under tumour cell influence, macrophageinduced angiogenesis is started when macrophages differentiate towards M2phenotype, the so-called angiogenic switch ${ }^{50}$. In hypoxic (tumour) areas, angiogenesis is promoted under influence of vascular endothelial growth factor (VEGF) produced by either CCL2- or HIF1 $\alpha$ - recruited macrophages ${ }^{39,51}$. Tissue remodelling occurs under influence of matrix metalloproteinases (MMPs). These are produced by TAM under influence of signals from surrounding cells, including tumour cells, (e.g. MMP2 and MMP9).

\subsection{Cancer metastases, the role of the macrophage}

Tumours consist of several different ecosystems, such as cancer nests, peritumoural stroma, perivascular regions and hypoxic regions. This results in a complex, developing organoid structure. The macrophage phenotype differs within these different areas, displaying distinct functions ${ }^{8}$. For proteolytic destruction of the matrix macrophages produce MMPs, (serine) proteases and cathepsins. Macrophages facilitate tumour cells to migrate through the dense stroma and to ultimately escape the basement membrane confinement (48). Metastases in breast cancer are attributed to perivascular macrophages producing endothelial growth factor (EGF) attracting macrophage-colony stimulating factor-producing cancer cells. This results in a coordinated migration and intravasation of cancer cells at macrophage dense sites. This migratory nature of perivascular macrophages is seen in vascular dense areas as opposed to limited TAM migration in avascular regions ${ }^{52,53}$. This combination of tumour cells and vessel-associated macrophages forming metastasis-favourable sites based on attracting cancer cells has been directly visualised in a murine model. It is hypothesised that these (vessel associated) TAM are able to promote migration and intravasation by chemotactically attracting tumour cells, and enabling them to enter the bloodstream ${ }^{52,53}$. The colony-stimulating factor 1 (CSF-1)/EGF paracrine loop, existing between tumour cells and macrophages, facilitates tumour cell migration along fibrous strands (fibrillar collagen I) in the stromal tissue towards blood vessels. Once the tumour cell arrives, the vascular 
macrophages facilitate further migration into the bloodstream ${ }^{54,55}$. In mice bearing mammary tumours, EGF or CSF-1 inhibition and genetic TAM-reduction effectively reduces circulating tumour cells ${ }^{48}$. In CRC mice models a collective invasion of sheets of cells are found instead of single cell invasion as in breast cancer. In CRC an immature myeloid cell population surrounding the invasive front is described. These immature myeloid cells produce MMP type 9 (MMP9) and type 2 (MMP2), which are required for tissue remodelling, and subsequent tumour cell invasion possibly resulting in metastase ${ }^{56}$. This macrophage-MMP9 production also reduces DCs ability to migrate and release anti-tumour activity ${ }^{57}$ in combination with the hypoxic environment. Upregulation of macrophageVEGF and basic fibroblast growth factor (bFGF) keeps T-cells from invading tumour environments by inhibiting adhesion molecule expression on endothelial cells $^{58}$. These are only a few examples of the total arsenal of the immunosuppressive repertoire regulated by tumours, orchestrating TAM to perform as is wanted. They use macrophage-capabilities, along with capabilities of other immune cells, to disrupt a healthy immune response. This review further confines itself to considering the role of the monocyte-macrophage continuum in cancer metastasis.

\subsection{Hybrids, the macrophage-cancer cell connection}

Another hypothesis states that macrophages are able to become malignantly invasive by integration of malignant DNA into the macrophage-host DNA. This process is called in-vivo fusion, which permanently transduces and transcribes macrophage-foreign DNA into the host cell ${ }^{59-61}$. Macrophages are also thought to either fuse with tumour cells, or to transfer migrational abilities onto them. The resulting hybrid cells exhibit metastatic abilities ${ }^{62-64}$. The specific macrophage traits such as aerobic glycolysis (the Warburg effect ${ }^{65-70}$ ), migratory and secretory abilities are retained after tumour cell macrophage fusion ${ }^{71}$. The hybrids use an autophagy-lysosomal pathway for sequestration and digestion of external food sources that are internalized via phagocytosis or other endocytic processes. These hybrids escape from regular immuno-surveillance by following this fusion/adoption pathway. Finally, a theory of tumour budding has been described in relation to metastases. Tumour budding is defined as single cells, or small clusters of up to 5 cells that are present in the peritumoural stromal tissue, (migrated) ahead of the invasive tumour front ${ }^{72,73}$. The buds show upregulation of metalloproteinases, urokinase receptors and cathepsin, which may contribute to increased migratory capacity and stromal invasion. 
Furthermore, they exhibit a low proliferative activity, which is in line with their migratory phenotype. Their production of MMP2, MMP7 and MMP9 are indicative of their matrix remodelling capabilities ${ }^{74}$.

\subsection{Macrophage diagnostics?}

The tumour budding observation could lead to metastases screening being based on an evaluation of the budding cells in the tumour biopsy or surgical resection specimen. Defining whether these cells are in fact hybrids or cells with incorporated tumour DNA could have prognostic value. For example, Kinouchi et al described the favourable disease free survival (DFS) in patients showing CD14+ macrophages at the invasive front. Better overall survival was found for the CD14high group opposed to the CD14Low group ${ }^{75}$. They also described dense infiltration with CD40+ TAM to indicate a favourable prognosis ${ }^{76}$. The Immunoscore for CRC by Galon ${ }^{77-79}$ investigates specific T-cell densities that has been claimed to have prognostic value. Including more cell types is thought to increase the performance of the score. The Klintrup-score ${ }^{80-82}$ focuses on the influx of more immune cells (such as neutrophils, granulocytes, lymphoid cells and macrophages) in the central tumour and its invasive border in postoperative specimens. Both the Immuno-score and Klintrup-score are based on pathological specimen as is the tumour budding evaluation. Although all of the above scores can inform clinicians about prognosis, the scores have no contribution to screening or follow-up settings because of the need for tissue investigation. Screening, monitoring or follow-up could become feasible if such an assay would require only minor invasive sampling of other materials, such as blood or excrements. To deliver additional value, any form of screening needs to be as little invasive as possible. When investigating (additional) discriminative power of a diagnostic technique, calculating the area under the curve (AUC) based on receiver operating characteristics analysis is an accepted instrument ${ }^{83}$. An example of a low-invasive diagnostic technique is the investigation of cytokine footprints. This technique uses a panel of cytokines to detect different inflammatory and neoplastic diseases. Kantola et al, investigated CRC patient discrimination from healthy individuals, resulting in an AUC of 0.890 based on these cytokine footprints. Regrettably, this trial omitted the comparison with groups consisting of patients with inflammatory or infectious diseases ${ }^{84}$. To use macrophages in a diagnostic setting, a peripheral blood based assay that covers a wide spectrum of colorectal diseases is needed. Subsequent introduction in the clinical setting is only possible after thorough validation. 


\section{Colorectal cancer}

\subsection{Epidemiology and risk factors}

$\mathrm{CRC}$ is currently the third most prevalent cancer worldwide. Combining new cases in men and women, CRC is even the second most common form of cancer after lung cancer ${ }^{85}$. In 2012, 13408 new cases of CRC were diagnosed in the Netherlands ${ }^{86,87}, 447.000$ in Europe ${ }^{88}$ and 1.4 million worldwide ${ }^{86,89}$. The expectation is that the incidence will rise to 2.4 million cases worldwide in 2035 ${ }^{88,89}$. Annual mortality in the Netherlands has continued to rise from around 4200 in 2002 to over 5000 patients in $2012^{86}$. Men are slightly more prone to develop $\mathrm{CRC}$ than women and most cases present themselves after the age of 55 years ${ }^{87}$. Age is the number one risk factor for development of sporadic CRC and over $90 \%$ of all CRC-cases develops after the first 50 years of life. Family history, inflammatory bowel disease, obesity, adult attained height, tobacco and alcohol abuse, high stress and western diet have also proven to be risk factors ${ }^{88-}$ 95. Physical activity, fruit and vegetable consumption and food high in dietary fibre are considered protective against developing $C R C^{94,96,97}$. One in every three CRC-cases in the Netherlands concerns rectal cancer $^{87}$. The lifetime risk in the Netherlands to be diagnosed with rectal cancer for a 35-year old male with a life expectancy of 85 years is 2.20 percent and for a female this percentage is 1.36 percent $^{86}$.

\subsection{Patient populations and pathogenesis}

CRC consists of hereditary (familial adenomatous polyposis (FAP), hereditary non polyposis CRC (HNPCC or Lynch's syndrome), MYH-associated polyposis (MAP), Peutz-Jegher's syndrome, juvenile coli (JPS)) and the non hereditary form, the sporadic CRC, which is the most common CRC type ${ }^{98}$. This review focuses on this last form of CRC. The sporadic cancer cases find their origin in evolving polyps from adenoma via dysplasia to carcinoma. The differentiation from adenoma to carcinoma takes about 10 years on average and is most often caused by multiple somatic mutations and genetic instability (such as MSI and various oncogene mutations) ${ }^{90,99}$. Epigenetic evaluation may lead to distinction between CRC types to identify favourable genetic profiles for certain treatment options. Currently only a few genomic markers are useful for prognosis. These include APC, MLH1 and MSH2, the latter two being known as mismatch repair (MMR) proteins. All three markers indicate a very high risk of CRC and guide surveillance frequency and prophylactic surgery recommendations ${ }^{100}$. In general, 
sporadic CRC with defective mismatch repair (dMMR) has a favourable prognosis compared to $\mathrm{CRC}$ with a germ line origin. Sporadic cancer cases are also detected in inflammatory bowel disease patients (IBD; Crohn's disease (CD) and ulcerative colitis (UC)). Although the CRC incidence in the IBD group equals 3 cases per 1000 patient years, risk does increase with disease duration and extent. Cumulative risk after 10 years of UC equals $1.6 \%$ and increases to $18.4 \%$ after 30 years of disease. In the IBD-patient group, CRC occurs mostly due to malignant epithelial changes during chronic and continuous reepithelisation processes as a response to repeated mucosal inflammation. In about $50 \%$ of the IBD-patient CRC-cases it concerns an interval-CRC, diagnosed or missed between initial screening and regular follow-up moments ${ }^{99}$. CRC-development in IBD patients may also follow the serrated neoplastic pathway, as an alternative origin of neoplasia ${ }^{101,102}$.

\subsection{Carcinoembryonic antigen in colorectal cancer}

In 1965 Gold and Freedman discovered the protein carcinoembryonic antigen $(C E A)^{103,104}$, a presumed colorectal-specific tumour marker at that time. First CEA was thought to be present only in fetal tissues in various stages of gestation $^{105}$. In the years following its discovery it became clear that the CEAantigen was found in other epithelial malignancies (e.g. lung, pancreatic, gastric, female reproductive tract ${ }^{105}$ and breast ${ }^{106}$ cancer) as well as in specific parts of normal tissue of the stomach, tongue, oesophagus, cervix, prostate and colon. Antibodies targeted against CEA were developed, but appeared to cross-react with normal antigens and CEA-related antigens. CEA is part of a 29-member family of genes and pseudogenes. Normal antigens consist of members such as CEA gene family members (CGMs) and pregnancy-specific $\beta-1$ glycoprotein (PSGs). CGM2 is closest in relation to CEA because of its presence in epithelial cells and no presence in granulocytes. Crossreacting antigens consist of nonspecific crossreacting antigens (NCAs), biliary glycoproteins (BGP), membraneassociated tissular antigen (MTA) and isomeric carcinoembryonic antigen $(C E A-S)^{107-109}$. Therefore the use of very specific CEA-antibodies, from the epitope groups 1-3 (Gold 1-3), is recommended ${ }^{107}$. Furthermore, elevated CEAlevels can be detected in selected benign situations such as an exacerbation of ulcerative colitis, (premalignant) adenomatous polyps ${ }^{108}$ and people who smoke tobacco. Serum CEA determination should therefore never be considered as a diagnostic assay on its own. Nowadays, it is common knowledge that CEA is a part of the intestinal barrier in the normal colon, with a limited tissue expression 
compared to malignancies ${ }^{110}$. In the colon CEA functions as a homophilic and heterophilic cell adhesion molecule with a heavily glycosylated protein structure containing a number of multi-antennary carbohydrate chains. CEA (and NCA) is presented on top of the columnar epithelial cells and released intra-luminally by the goblet cells in the mucinous layer on top of the glycocalyx. The apical glycocalyx is the microvillus region on top of the mature enterocytes, also called the "fuzzy coat". New glycocalyx is continuously formed at the apical surface of these mature enterocytes. Replacing the microorganism-saturated 'old' glycocalyx is regulated by inflammatory cytokines. Direct interactions of CEA with microorganisms have been described, preventing the microorganisms from reaching down to the microvilli of the epithelial cells to invade them. CEA is able (together with BGP and NCA) to bind and trap these microorganisms, and furthermore is thought to have a signalling function to the CEA-expressing cells below $^{109}$.
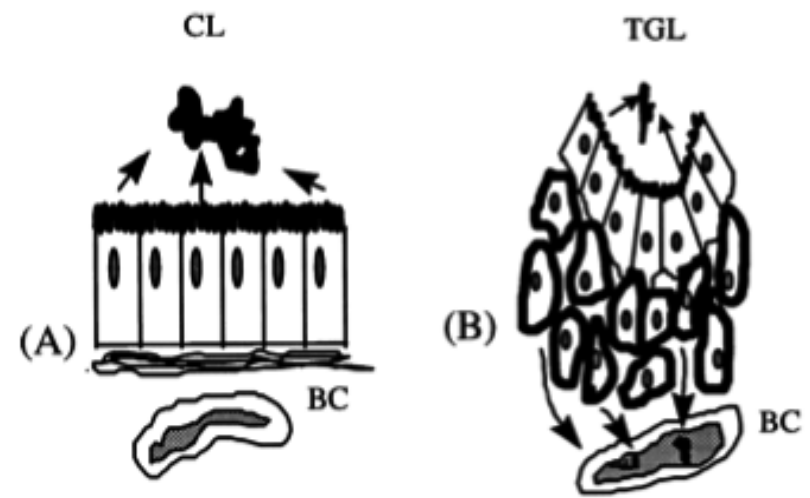

Figure 1.3 CEA-excretion in normal colon and colon cancer. A: CEA is released into the colon lumen $(C L)$ by polarised columnar epithelial cells and not into the blood capillaries (BC). $B$ : CEA is released by non-polarised neoplastic cells into tumour gland lumen (TGL), located deep inside tumour glands. CEA enters the blood stream via access to lymph and blood vessels through intercellular spaces (Hammarström 1999).

The applicability of CEA in a diagnostic or screening setting is limited to an additional role besides the primary assessment of clinical symptoms, imaging techniques and colonoscopy with biopsy ${ }^{111-114}$. Current clinical applications are surveillance for local recurrence or (hepatic) metastasis, prognosis of especially stage II patients after CRC-resection and monitoring of advanced disease ${ }^{114,115}$. 
Other biomarkers for CRC screening, prognosis or response such as TIMP-1 ${ }^{116}$ and Septin $9^{117-119}$, are not commonly implemented. Emerging CRC biomarkers include multi-gene profiles, CA-19.9, stool DNA-profiles and transcription factor AP-2 epsilon (TFAP2E) hypermethylation ${ }^{120}$. The latter is used for chemotherapy response prediction ${ }^{114}$.

\subsection{Currently available screening methods for CRC}

Currently, the gold standard for CRC diagnosis remains colonoscopy combined with biopsy, after which collected tissue is subject to pathology evaluation ${ }^{85}$. Colonoscopy is not without risks (e.g. perforation $(0.07 \%)$, bleeding $(1.34 \%)$ ). Besides this, it is also uncomfortable for patients. Even high quality imaging techniques in current settings are not able to fill in the need for tissue or cell collection. Moreover, (additional) radiation burden remains and costs are relatively high. Detecting aberrant DNA methylation in faecal DNA in CRC or advanced adenomas has a sensitivity of 46 to $77 \%$ for detecting early stage $\mathrm{CRC}$. It was recently added to cancer-screening guidelines of the ACS and is currently under investigation in the Netherlands (see below). This technique is superior to the faecal occult blood test (guaciaFOBT or gFOBT). Faecal Immunochemical Test (FIT or iFOBT) has many advantages over gFOBT, such as better analytical sensitivity and specificity and adjustable cut-off concentration. It has therefore become the preferred faecal test ${ }^{85,114}$. Positive outcomes in all FOBT-test types need diagnosis confirmation by colonoscopy. In screening, colonoscopy is the gold standard, although this has never been evaluated in a large randomized controlled trial ${ }^{85}$. Currently a large populationbased screening trial has started in the Netherlands. It is planned to include 891.203 persons above 50 years old in 2013-2014. The screening program is aimed at diagnosing $\mathrm{CRC}$ and advanced adenomas (AAD). Eligible persons are invited for iFOBT-screening and in case of a positive result (iFOBT result above cut-off value), a routine colonoscopy is offered. In this trial so far $n=11.430$ colonoscopies have been performed. Preliminary results show a CRC/AAD detection ratio of 35.5 per 1000 (equals 1 in 28) in 129.395 screened participants until now ${ }^{121}$. iFOBT showed a $59.8 \%$ false-positive rate (meaning no CRC or AAD) at colonoscopy. In $31.9 \%$ of the persons with positive iFOBT-tests colonoscopy did not reveal any adenomas at all. In $60.1 \%$ of the persons who underwent colonoscopy a form of adenoma was discovered, some of which were eligible for surveillance colonoscopy programmes. 
Molecular tumour features of the serrated pathway might be involved in CRC development within 5 years after colonoscopy ${ }^{122}$. Drawbacks are that colonoscopy does not prevent deaths in general and has a 1 in 3 chance of false-positive outcomes. Besides the high costs, risks of screening are overdiagnosis, negative psychosocial consequences, somatic complications, the use of invasive follow-up procedures and surveillance withdrawal because of adverse effects. Maybe most relevant when considering screening, is that is offered to mostly healthy individuals without a wish to solve a health problem. The interventions can cause serious harm with only modest or no reduction in cancer related mortality. Therefore a benefit for some will come at the expense of harm to others ${ }^{123}$.

\subsection{New diagnostic techniques}

Recently new minimal invasive techniques such as liquid biopsy and detecting tumour related material in macrophages were investigated. The first technique, liquid biopsy, is a method to detect circulating tumour DNA (ctDNA) and circulating tumour cells (CTC) in peripheral blood ${ }^{124}$. Both are shedded from the tumour into the blood stream. Regular tissue biopsies are not reflective of the whole spectrum of aberrant cells in for instance breast cancer; the assessment of tumour heterogeneity is proposed to be more accurate using liquid biopsies $^{125}$. These techniques show it is possible to monitor genes and changes thereof, associated with certain treatment sensitivity, such as anti-EGFRtreatment. Changes in gene-expression (such as KRAS, BRAF, PIK3CA and EGFR) indicate possible treatment failure and enable treatment alteration ${ }^{126-128}$. The challenge with ctDNA and CTC analyses is that it is mostly investigated in metastatic disease cases, where the load of CTCs and ctDNA is the highest $^{129,130}$. In early disease stages this is not the case. Detection of tumour related material in macrophages has been investigated in prostate cancer, using commonly known tumour markers (such as prostate specific antigen (PSA)) in a new way, showing possibilities in detecting prostate cancer already in early stages $^{30,131}$. Both described techniques are currently under further investigation and the latter is being investigated in relation to $C R C^{132}$.

\subsection{Validation of new diagnostic techniques}

Investigating diagnostic methods also implies validating them. Besides validity, steps necessary to ensure reliability and efficacy of tumour marker data in the overall care and management of patients need to be investigated. Laboratory 
assay validation consists of a pre-analytic, analytic and post-analytic phase ${ }^{133,134}$. During the pre-analytic phase test ordering, sample collection, transport and storage conditions, laboratory sample delivery and preparation and handling before analysis are assessed. The analytic phase consists of choice of method, standardisation, calibration and precision assessment. Postanalytical phase assessment includes result presentation, result interpretation and comparison to other clinical and laboratory data and finally using the collected data in disease management. If the assessed items show good reproducibility, a test can be considered properly validated ${ }^{134}$. Biological variation within and between patients is an item not automatically investigated using the above definition of validity analysis. This type of variation is important, although not always part of regular investigations in experimental (tumour marker) assays. For example, total variation up to $35 \%$ (biological and analytical) within a patient in sequential leucocyte analyses is not uncommon ${ }^{135}$. Normally, such a variation is clinically investigated to exclude serious underlying pathology. Variability in tumour marker assays is common as well. Therefore, in serum tumour marker analysis a baseline level increase of $25-30 \%$ occurring twice in a row is considered clinically relevant ${ }^{111,136}$. Additional diagnostic investigation for detection of recurrent or metastatic disease is warranted when such an increase is discovered.

\section{Clinical application of macrophage-diagnostics in colorectal cancer}

\subsection{Screening and diagnostic application in colorectal cancer}

Based on the work of Herwig et al. ${ }^{28,29,137}$ and Leers et al. ${ }^{30}$ on PSA detection in macrophages in prostate cancer patients, the concept of the method was adapted to CRC in 2008 using CEA as tumour marker-directed primary antibody. In a pilot study CRC patients were investigated for presence of monocytes/ macrophages exhibiting intracellular (tumour-related) debris, such as CEA on the debris. The pilot study reported that it was possible to intracellularly detect CEA in activated macrophages. It further showed a discriminating potential for distinguishing CRC patients with AJCC stages I-IV from healthy individuals and patients with benign inflammatory disease ${ }^{132}$. This technique is potentially of value in different settings for diagnostic or screening purposes, in follow-up after CRC surgery and to monitor treatment effects. A thorough elementary investigation was started to gain better understanding of the cellular basis ${ }^{138,139}$, 
the technique pit-falls ${ }^{140}$ and possible clinical implications in therapy monitoring (unpublished results).

Screening for CRC is a hot topic in medicine as mentioned in paragraph 1.3. In order to add an experimental method to current standard diagnostic trajectories in CRC a series of steps needs to be taken. Validation of such an assay is key. Kievit et al. ${ }^{112}$ described the additional value of regular CEA-analysis in the follow-up setting. They showed clearly it only has benefit in specific patient groups and only in follow-up setting, not in a diagnostic setting.

\subsection{Monitoring neoadjuvant treatment in rectal cancer}

Monitoring inflammation based on monocyte levels has been done earlier in different settings such as erysipelas and pneumonia ${ }^{141,142}$. Applying inflammatory monitoring in rectal cancer should be based on the inflammatory component in the tumour and its surroundings. Elevation of the level of inflammatory cells in the peripheral blood could reflect the presence of a local immune reaction. Using a macrophage assay in a neoadjuvant treatment monitoring setting could facilitate optimisation of treatment by treatment alteration. At this moment rectal cancer patients are only monitored for this purpose in trial setting, mainly focussing on imaging techniques for this purpose, such as PET-CT and MRI. Both are used for staging and restaging purposes, important for preoperative assessment of tumour regression after neoadjuvant radiochemotherapy ${ }^{143}$. Monitoring treatment efficacy using blood biomarkers, such as CEA and IL8, complimentary to imaging techniques was recently published by Buijsen et al. ${ }^{144}$. They showed response predicting capabilities for serum-based biomarkers in relation to pathological complete response ( $p C R)$ as the outcome measure. 


\section{Aim of this thesis}

This body of knowledge and the results of a pilot study, investigating the feasibility of detection of CEA in activated macrophages, evolved into a PhDtrajectory that has finally resulted in this thesis. The aim of this $\mathrm{PhD}$ trajectory consisted of multiple parts that are described in the following paragraphs. The role of macrophages in CRC, in cancer in general and their possible diagnostic applications has been investigated.

\subsection{Macrophages in CRC}

To investigate if phagocytes could internalise tumour-related material by immunophenotypical detection of it, we conducted two studies using the CRCmodel as our investigational platform. A wider arsenal of tumour-related material was investigated beside CEA, including TKTL1, M30 and CK. Confirmation of the presence of the above-mentioned tumour-related material and the possible location inside the phagocyte has been described in chapter 2 and 3 using light microscopy and electron microscopy respectively.

\subsection{Proof of principle}

The internalisation of tumour related material by macrophages causing a form of concentration of this material inside the macrophage was translated from the prostate cancer method $29,30,131,137,145$ into a CRC method. CEA, the most common tumour-marker for CRC, was investigated using this experimental method. The detailed results of this proof of principle investigation are described in chapter 4.

\subsection{Validation of diagnostic test}

Validation of tumour related material detection in macrophages is a major portion of this thesis. It has been referred to as epitope detection, because a wider arsenal of tumour related material (TKTL1, M30, CK and CEA) has been investigated to prove that its reproducibility isn't limited to CEA. Without a proper validation trajectory, clinical application of a new (macrophage) assay in any form is not possible ${ }^{111,133,136,146}$. The validation trajectory has proven that a macrophage assay for diagnostic purposes is technically feasible ${ }^{140}$, as has been described in chapter 5 . 


\subsection{Early clinical application of an experimental diagnostic test}

To investigate possible clinical application a population of rectal cancer patients was chosen for three reasons. Firstly, in our institution this population is carefully monitored during pre-operative radiochemotherapy using established modalities such as blood biomarkers, PET-CT and MRI ${ }^{144,147-151}$. This created the opportunity to investigate the performance of a newly developed test in this carefully controlled disease-environment. Secondly, it was possible to evaluate the new test's performance in comparison to the above-mentioned modalities and to investigate possible additional value to response prediction models. Thirdly, the population was chosen due to the expectation that neoadjuvant treatment causes the highest possible yield of shedding of tumour related material. This means that the new diagnostic tool should be able to detect baseline levels and baseline level changes during treatment. This could then be evaluated in relation to different relevant definitions of outcome in this population. The results of this early diagnostic application have been described in chapter 6.

\subsection{Discussion, future developments, challenges and recommendations}

In the discussion all results are discussed using literature describing possible explanations for the results and their value. Also additional (non published) findings will be discussed. Finally, future developments, challenges and recommendations for future research are postulated. 


\section{References}

1. Chow A, Brown BD, Merad M. Studying the mononuclear phagocyte system in the molecular age. Nat Rev Immunol 2011;11:788-98.

2. Tauber Al. Metchnikoff and the phagocytosis theory. Nat Rev Mol Cell Biol 2003;4:897-901.

3. Ohtani H, Naito Y, Saito K, Nagura H. Expression of costimulatory molecules B7-1 and B7-2 by macrophages along invasive margin of colon cancer: A possible antitumor immunity? Lab Invest 1997;77:231-41.

4. Jenkins SJ, Ruckerl D, Cook PC, Jones LH, Finkelman FD, Rooijen NV, et al. Local macrophage proliferation, rather than recruitment from the blood, is a signature of $\mathrm{TH}_{2}$ inflammation. Science 2011;332:1284-8.

5. Geissmann F, Jung S, Littman DR. Blood monocytes consist of two principal subsets with distinct migratory properties. Immunity 2003;19:71-82.

6. Dalton HJ, Armaiz-Pena GN, Gonzalez-Villasana V, Lopez-Berestein G, Bar-Eli M, Sood AK. Monocyte subpopulations in angiogenesis. Cancer Res 2014;74:1287-93.

7. Gomez Perdiguero E, Geissmann F. Cancer immunology. Identifying the infiltrators. Science 2014;344:801-2.

8. Van Overmeire E, Laoui D, Keirsse J, Van Ginderachter JA, Sarukhan A. Mechanisms driving macrophage diversity and specialization in distinct tumor microenvironments and parallelisms with other tissues. Front Immunol 2014;5:127.

9. Wagner S, Czub S, Greif M, Vince GH, Süss N, Kerkau S, et al. Microglial/macrophage expression of interleukin 10 in human glioblastomas. Int J Cancer 1999;82:12-6.

10. Sica A, Saccani A, Bottazzi B, Polentarutti N, Vecchi A, van Damme J, Mantovani A. Autocrine production of IL-10 mediates defective IL-12 production and nf-kappa B activation in tumorassociated macrophages. J Immunol 2000;164:762-7.

11. Mosser DM, Edwards JP. Exploring the full spectrum of macrophage activation. Nat Rev Immunol 2008;8:958-69.

12. Zigmond $\mathrm{E}$, Jung $\mathrm{S}$. Intestinal macrophages: Well educated exceptions from the rule. Trends Immunol 2013;34:162-8.

13. Ziegler-Heitbrock HW, Fingerle G, Strobel M, Schraut W, Stelter F, Schutt C, et al. The novel subset of CD14+/CD16+ blood monocytes exhibits features of tissue macrophages. Eur J Immunol 1993;23:2053-8.

14. Perussia B, Trinchieri G, Jackson A, Warner NL, Faust J, Rumpold H, et al. The fc receptor for igg on human natural killer cells: Phenotypic, functional, and comparative studies with monoclonal antibodies. J Immunol 1984;133:180-9.

15. Rumpold H, Kraft D, Obexer G, Böck G, Gebhart W. A monoclonal antibody against a surface antigen shared by human large granular lymphocytes and granulocytes. J Immunol 1982; 129:1458-64.

16. Fingerle G, Pforte A, Passlick B, Blumenstein M, Strobel M, Ziegler-Heitbrock HW. The novel subset of CD14+/CD16+ blood monocytes is expanded in sepsis patients. Blood 1993;82:3170-6.

17. Saleh MN, Goldman SJ, LoBuglio AF, Beall AC, Sabio H, McCord MC, et al. CD16+ monocytes in patients with cancer: Spontaneous elevation and pharmacologic induction by recombinant human macrophage colony-stimulating factor. Blood 1995;85:2910-7.

18. Wong KL, Yeap WH, Tai JJ, Ong SM, Dang TM, Wong SC. The three human monocyte subsets: Implications for health and disease. Immunol Res 2012;53:41-57.

19. Ziegler-Heitbrock L. Monocyte subsets in man and other species. Cell Immunol 2014;289: 135-9.

20. Ziegler-Heitbrock L, Ancuta P, Crowe S, Dalod M, Grau V, Hart DN, et al. Nomenclature of monocytes and dendritic cells in blood. Blood 2010;116:e74-80. 
21. Jung S, Aliberti J, Graemmel P, Sunshine MJ, Kreutzberg GW, Sher A, Littman DR. Analysis of fractalkine receptor $\mathrm{CX}(3) \mathrm{CR} 1$ function by targeted deletion and green fluorescent protein reporter gene insertion. Mol Cell Biol 2000;20:4106-14.

22. Auffray C, Fogg D, Garfa M, Elain G, Join-Lambert O, Kayal S, et al. Monitoring of blood vessels and tissues by a population of monocytes with patrolling behavior. Science 2007;317:666-70.

23. Carlin LM, Auffray C, Geissmann F. Measuring intravascular migration of mouse ly6c(low) monocytes in vivo using intravital microscopy. Curr Protoc Immunol 2013;Chapter 14:Unit 14.33.1-16.

24. Jakubzick C, Gautier E, Gibbings S, Sojka D, Schlitzer A, Johnson T, et al. Minimal differentiation of classical monocytes as they survey steady-state tissues and transport antigen to lymph nodes. Immunity 2013;39:599-610.

25. Gerrity RG. The role of the monocyte in atherogenesis: II. Migration of foam cells from atherosclerotic lesions. Am J Pathol 1981;103:191-200.

26. Joly S, Francke M, Ulbricht E, Beck S, Seeliger M, Hirrlinger P, et al. Cooperative phagocytes: Resident microglia and bone marrow immigrants remove dead photoreceptors in retinal lesions. Am J Pathol 2009;174:2310-23.

27. Eter N, Engel DR, Meyer L, Helb HM, Roth F, Maurer J, et al. In vivo visualization of dendritic cells, macrophages, and microglial cells responding to laser-induced damage in the fundus of the eye. Invest Ophthalmol Vis Sci 2008;49:3649-58.

28. Herwig R. Measurement of intracellular versus extracellular prostate-specific antigen levels in peripheral macrophages: A new approach to noninvasive diagnosis of prostate cancer. Clin Prostate Cancer 2004;3:184-8..

29. Herwig R, Horninger W, Rehder P, Klocker H, Ramoner R, Thurnher M, et al. Ability of psapositive circulating macrophages to detect prostate cancer. The Prostate 2005;62:290-8.

30. Leers MPG, Nap M, Herwig R, Delaere K, Nauwelaers F. Circulating psa-containing macrophages as a possible target for the detection of prostate cancer. Am J Clin Pathol 2008;129:649.

31. De Kleer I, Willems F, Lambrecht B, Goriely S. Ontogeny of myeloid cells. Front Immunol 2014;5:423.

32. Ginhoux F, Greter M, Leboeuf M, Nandi S, See P, Gokhan S, et al. Fate mapping analysis reveals that adult microglia derive from primitive macrophages. Science 2010;330:841-5.

33. Hashimoto D, Chow A, Noizat C, Teo P, Beasley MB, Leboeuf M, et al. Tissue-resident macrophages self-maintain locally throughout adult life with minimal contribution from circulating monocytes. Immunity 2013;38:792-804.

34. Yona S, Kim K-W, Wolf $\mathrm{Y}$, Mildner A, Varol D, Breker M, et al. Fate mapping reveals origins and dynamics of monocytes and tissue macrophages under homeostasis. Immunity 2013; 38:79-91.

35. Bain C, Scott L, Uronen-Hansson H, Gudjonsson S, Jansson O, Grip O, et al. Resident and pro-inflammatory macrophages in the colon represent alternative context-dependent fates of the same ly6chi monocyte precursors. Mucosal Immunology 2013;6:498-510.

36. Guilliams M, De Kleer I, Henri S, Post S, Vanhoutte L, De Prijck S, et al. Alveolar macrophages develop from fetal monocytes that differentiate into long-lived cells in the first week of life via GM-CSF. J Exp Med 2013;210:1977-92.

37. Chao MP, Alizadeh AA, Tang C, Myklebust JH, Varghese B, Gill S, et al. Anti-CD47 antibody synergizes with rituximab to promote phagocytosis and eradicate non-hodgkin lymphoma. Cell 2010;142:699-713.

38. Zhang QW, Liu L, Gong $\mathrm{CY}$, Shi HS, Zeng YH, Wang XZ, et al. Prognostic significance of tumor-associated macrophages in solid tumor: A meta-analysis of the literature. PLoS One 2012; 7:e50946.

39. Lin EY, Pollard JW. Tumor-associated macrophages press the angiogenic switch in breast cancer. Cancer Res 2007;67:5064-6. 
40. Schreiber RD, Old LJ, Smyth MJ. Cancer immunoediting: Integrating immunity's roles in cancer suppression and promotion. Science 2011;331:1565-70.

41. Dunn GP, Bruce AT, Ikeda H, Old LJ, Schreiber RD. Cancer immunoediting: From immunosurveillance to tumor escape. Nat Immunol 2002;3:991-8.

42. Vesely MD, Kershaw MH, Schreiber RD, Smyth MJ. Natural innate and adaptive immunity to cancer. Annu Rev Immunol 2011;29:235-71.

43. Saccani A, Schioppa T, Porta C, Biswas SK, Nebuloni M, Vago L, et al. P50 nuclear factorkappab overexpression in tumor-associated macrophages inhibits M1 inflammatory responses and antitumor resistance. Cancer Res 2006;66:11432-40.

44. Biswas SK, Gangi L, Paul S, Schioppa T, Saccani A, Sironi M, et al. A distinct and unique transcriptional program expressed by tumor-associated macrophages (defective nf-kappab and enhanced IRF-3/STAT1 activation). Blood 2006;107:2112-22.

45. Mantovani A, Sozzani S, Locati M, Allavena P, Sica A. Macrophage polarization: Tumorassociated macrophages as a paradigm for polarized M2 mononuclear phagocytes. Trends Immunol 2002;23:549-55.

46. Kawamura K, Komohara Y, Takaishi K, Katabuchi H, Takeya M. Detection of M2 macrophages and colony-stimulating factor 1 expression in serous and mucinous ovarian epithelial tumors. Pathol Int 2009;59:300-5.

47. Sugai $H$, Kono K, Takahashi A, Ichihara F, Kawaida H, Fuji H, Matsumoto $Y$. Characteristic alteration of monocytes with increased intracellular IL-10 and IL-12 in patients with advancedstage gastric cancer. J Surg Res 2004;116:277-87.

48. Qian BZ, Pollard JW. Macrophage diversity enhances tumor progression and metastasis. Cell 2010;141:39-51.

49. Zaynagetdinov R, Sherrill TP, Polosukhin VV, Han W, Ausborn JA, McLoed AG, et al. A critical role for macrophages in promotion of urethane-induced lung carcinogenesis. J Immunol 2011;187:5703-11.

50. Hanahan D, Folkman J. Patterns and emerging mechanisms of the angiogenic switch during tumorigenesis. Cell 1996;86:353-64.

51. Lin EY, Li JF, Gnatovskiy L, Deng Y, Zhu L, Grzesik DA, et al. Macrophages regulate the angiogenic switch in a mouse model of breast cancer. Cancer Res 2006;66:11238-46.

52. Lewis CE, Pollard JW. Distinct role of macrophages in different tumor microenvironments. Cancer Res 2006;66:605-12.

53. Wyckoff JB, Wang Y, Lin EY, Li JF, Goswami S, Stanley ER, et al. Direct visualization of macrophage-assisted tumor cell intravasation in mammary tumors. Cancer Res 2007;67: 2649-56.

54. Condeelis J, Segall JE. Intravital imaging of cell movement in tumours. Nat Rev Cancer 2003;3:921-30.

55. Wang W, Goswami S, Sahai E, Wyckoff JB, Segall JE, Condeelis JS. Tumor cells caught in the act of invading: Their strategy for enhanced cell motility. Trends Cell Biol 2005;15:138-45.

56. Kitamura $T$, Kometani $K$, Hashida $H$, Matsunaga A, Miyoshi $H$, Hosogi $H$, et al. SMAD4deficient intestinal tumors recruit CCR1+ myeloid cells that promote invasion. Nat Genet 2007;39:467-75.

57. Zhao $P$, Li XG, Yang M, Shao Q, Wang D, Liu S, et al. Hypoxia suppresses the production of MMP-9 by human monocyte-derived dendritic cells and requires activation of adenosine receptor a2b via camp/PKA signaling pathway. Mol Immunol 2008;45:2187-95.

58. Motz GT, Coukos G. Deciphering and reversing tumor immune suppression. Immunity 2013; 39:61-73.

59. Goldenberg DM. Horizontal transmission of malignancy by cell-cell fusion. Expert Opin Biol Ther 2012;12 Suppl 1:S133-9.

60. Goldenberg DM, Gold DV, Loo M, Liu D, Chang CH, Jaffe ES. Horizontal transmission of malignancy: In-vivo fusion of human lymphomas with hamster stroma produces tumors retaining human genes and lymphoid pathology. PLoS One 2013;8:e55324. 
61. Goldenberg DM, Rooney RJ, Loo M, Liu D, Chang $\mathrm{CH}$. In-vivo fusion of human cancer and hamster stromal cells permanently transduces and transcribes human DNA. PLoS One 2014; 9:e107927.

62. Pawelek JM, Chakraborty AK. The cancer cell--leukocyte fusion theory of metastasis. Adv Cancer Res 2008;101:397-444.

63. Pawelek JM, Chakraborty AK. Fusion of tumour cells with bone marrow-derived cells: A unifying explanation for metastasis. Nat Rev Cancer 2008;8:377-86.

64. Pawelek JM. Fusion of bone marrow-derived cells with cancer cells: Metastasis as a secondary disease in cancer. Chin J Cancer 2014;33:133-9.

65. Grimm M, Schmitt S, Teriete P, Biegner T, Stenzl A, Hennenlotter J, et al. A biomarker based detection and characterization of carcinomas exploiting two fundamental biophysical mechanisms in mammalian cells. BMC Cancer 2013;13:569.

66. Xu X, Zur Hausen A, Coy JF, Löchelt M. Transketolase-like protein 1 (TKTL1) is required for rapid cell growth and full viability of human tumor cells. Int J Cancer 2009;124:1330-7.

67. Demetrius LA, Coy JF, Tuszynski JA. Cancer proliferation and therapy: The warburg effect and quantum metabolism. Theor Biol Med Model 2010;7:2.

68. Langbein S, Zerilli M, Zur Hausen A, Staiger W, Rensch-Boschert K, Lukan N, et al. Expression of transketolase TKTL1 predicts colon and urothelial cancer patient survival: Warburg effect reinterpreted. Br J Cancer 2006;94:578-85.

69. Wittig R, Coy JF. The role of glucose metabolism and glucose-associated signalling in cancer. Perspect Medicin Chem 2008;1:64-82.

70. Fritz P, Coy JF, Mürdter TE, Ott G, Alscher MD, Friedel G. TKTL-1 expression in lung cancer. Pathol Res Pract 2012;208:203-9.

71. Lazova R, Chakraborty A, Pawelek JM. Leukocyte-cancer cell fusion: Initiator of the warburg effect in malignancy? In: Adv Exp Med Biol. United States: 2011:151-72.

72. Koelzer VH, Langer R, Zlobec I, Lugli A. Tumor budding in upper gastrointestinal carcinomas. Front Oncol 2014;4:216.

73. Satoh K, Nimura S, Hamasaki M, Aoki M, Koga K, Iwasaki H, et al. Tumor budding in colorectal carcinoma assessed by cytokeratin immunostaning and budding areas: Possible involvement of c-met. Cancer Sci 2014;105:1487-95.

74. Hostettler L, Zlobec I, Terracciano L, Lugli A. ABCG5-positivity in tumor buds is an indicator of poor prognosis in node-negative colorectal cancer patients. World J Gastroenterol 2010;16: 732-9.

75. Kinouchi M, Miura K, Mizoi T, Ishida K, Fujibuchi W, Ando T, et al. Infiltration of cd14-positive macrophages at the invasive front indicates a favorable prognosis in colorectal cancer patients with lymph node metastasis. Hepatogastroenterology 2011;58:352-8.

76. Kinouchi M, Miura K, Mizoi T, Ishida K, Fujibuchi W, Sasaki H, et al. Infiltration of cd40-positive tumor-associated macrophages indicates a favorable prognosis in colorectal cancer patients. Hepatogastroenterology 2013;60:83-8.

77. Angell H, Galon J. From the immune contexture to the immunoscore: The role of prognostic and predictive immune markers in cancer. Current Opinion in Immunology 2013;25:261-7.

78. Galon J, Angell H, Bedognetti D, Marincola $F$. The continuum of cancer immunosurveillance: Prognostic, predictive, and mechanistic signatures. Immunity 2013;39:11-26.

79. Galon J, Fridman WH, Pages F. The adaptive immunologic microenvironment in colorectal cancer: A novel perspective. Cancer Res 2007;67:1883-6.

80. Klintrup K, Mäkinen JM, Kauppila S, Väre PO, Melkko J, Tuominen H, et al. Inflammation and prognosis in colorectal cancer. European Journal of Cancer 2005;41:2645-54.

81. Roxburgh CS, Salmond JM, Horgan PG, Oien KA, McMillan DC. Tumour inflammatory infiltrate predicts survival following curative resection for node-negative colorectal cancer. European Journal of Cancer 2009;45:2138-45. 
82. Powell AG, Ferguson J, Al-Mulla F, Orange C, McMillan DC, Horgan PG, et al. The relationship between genetic profiling, clinicopathological factors and survival in patients undergoing surgery for node-negative colorectal cancer: 10-year follow-up. J Cancer Res Clin Oncol 2013; 139:2013-20.

83. DeLong ER, DeLong DM, Clarke-Pearson DL. Comparing the areas under two or more correlated receiver operating characteristic curves: A nonparametric approach. Biometrics 1988;44:837-45.

84. Kantola T, Klintrup K, Väyrynen JP, Vornanen J, Bloigu R, Karhu T, et al. Stage-dependent alterations of the serum cytokine pattern in colorectal carcinoma. Br J Cancer 2012;107: 1729-36.

85. Duffy MJ, van Rossum LG, van Turenhout ST, Malminiemi O, Sturgeon C, Lamerz R, et al. Use of faecal markers in screening for colorectal neoplasia: A european group on tumor markers position paper. Int J Cancer 2011;128:3-11.

86. Cijfers over kanker, 2001-2012. IKNL.

87. Colorectaalcarcinoom richtlijn 2014 version 3.0. IKNL.

88. Ferlay J, Steliarova-Foucher E, Lortet-Tieulent J, Rosso S, Coebergh JW, Comber H, et al. Cancer incidence and mortality patterns in europe: Estimates for 40 countries in 2012. Eur J Cancer 2013;49:1374-403.

89. Colorectal cancer incidence worldwide 2012. WRCF.org.

90. Ahmed S, Johnson K, Ahmed O, lqbal N. Advances in the management of colorectal cancer: From biology to treatment. Int J Colorectal Dis 2014;29:1031-42.

91. Rohrmann S, Linseisen J, Jakobsen MU, Overvad K, Raaschou-Nielsen O, Tjonneland A, et al. Consumption of meat and dairy and lymphoma risk in the european prospective investigation into cancer and nutrition. Int J Cancer 2011;128:623-34.

92. Bergmann MM, Schütze $M$, Steffen A, Boeing $H$, Halkjaer J, Tjonneland $A$, et al. The association of lifetime alcohol use with measures of abdominal and general adiposity in a largescale european cohort. Eur J Clin Nutr 2011;65:1079-87.

93. Chan DS, Lau R, Aune D, Vieira R, Greenwood DC, Kampman E, Norat T. Red and processed meat and colorectal cancer incidence: Meta-analysis of prospective studies. PLoS One 2011; 6:e20456.

94. Murphy N, Norat T, Ferrari P, Jenab M, Bueno-de-Mesquita B, Skeie G, et al. Dietary fibre intake and risks of cancers of the colon and rectum in the european prospective investigation into cancer and nutrition (EPIC). PLoS One 2012;7:e39361.

95. Ferrari $\mathrm{P}$, Licaj I, Muller DC, Kragh Andersen $\mathrm{P}$, Johansson $\mathrm{M}$, Boeing $\mathrm{H}$, et al. Lifetime alcohol use and overall and cause-specific mortality in the european prospective investigation into cancer and nutrition (EPIC) study. BMJ Open 2014;4:e005245.

96. Aune D, Lau R, Chan DS, Vieira R, Greenwood DC, Kampman E, Norat T. Nonlinear reduction in risk for colorectal cancer by fruit and vegetable intake based on meta-analysis of prospective studies. Gastroenterology 2011;141:106-18.

97. Steins Bisschop CN, van Gils CH, Emaus MJ, Bueno-de-Mesquita HB, Monninkhof EM, Boeing $\mathrm{H}$, et al. Weight change later in life and colon and rectal cancer risk in participants in the EPICPANACEA study. Am J Clin Nutr 2014;99:139-47.

98. Centelles JJ. General aspects of colorectal cancer. ISRN Oncol 2012;2012:139268.

99. Sanduleanu S, Rutter MD. Interval colorectal cancers in inflammatory bowel disease: The grim statistics and true stories. Gastrointest Endosc Clin N Am 2014;24:337-48.

100. Markowitz SD, Bertagnolli MM. Molecular origins of cancer: Molecular basis of colorectal cancer. N Engl J Med 2009;361:2449-60.

101. Bogaert J, Prenen H. Molecular genetics of colorectal cancer. Ann Gastroenterol 2014;27:9-14.

102. Kim ER, Chang DK. Colorectal cancer in inflammatory bowel disease: The risk, pathogenesis, prevention and diagnosis. World J Gastroenterol 2014;20:9872-81.

103. Gold P, Freedman SO. Demonstration of tumor-specific antigens in human colonic carcinomata by immunological tolerance and absorption techniques. J Exp Med 1965;121:439-62. 
104. Gold P, Freedman SO. Specific carcinoembryonic antigens of the human digestive system. J Exp Med 1965;122:467-81.

105. Nap M, Mollgard K, Burtin P, Fleuren GJ. Immunohistochemistry of carcino-embryonic antigen in the embryo, fetus and adult. Tumour Biol 1988;9:145-53.

106. Nap M, Keuning $H$, Burtin $P$, Oosterhuis JW, Fleuren G. CEA and NCA in benign and malignant breast tumors. Am J Clin Pathol 1984;82:526-34.

107. Nap M, Hammarström ML, Börmer O, Hammarström S, Wagener C, Handt S, et al. Specificity and affinity of monoclonal antibodies against carcinoembryonic antigen. Cancer Res 1992;52: 2329-39.

108. Martin EW, Kibbey WE, DiVecchia L, Anderson G, Catalano P, Minton JP. Carcinoembryonic antigen: Clinical and historical aspects. Cancer 1976;37:62-81.

109. Hammarström $S$. The carcinoembryonic antigen (CEA) family: Structures, suggested functions and expression in normal and malignant tissues. Semin Cancer Biol 1999;9:67-81.

110. Goldstein MJ, Mitchell EP. Carcinoembryonic antigen in the staging and follow-up of patients with colorectal cancer. Cancer Investigation 2005;23:338.

111. Sturgeon C. Practice guidelines for tumor marker use in the clinic. Clin Chem 2002;48:1151.

112. Kievit J. Follow-up of patients with colorectal cancer: Numbers needed to test and treat. Eur J Cancer 2002;38:986-99.

113. Duffy MJ, van Dalen A, Haglund C, Hansson L, Holinski-Feder E, Klapdor R, et al. Tumour markers in colorectal cancer: European group on tumour markers (EGTM) guidelines for clinical use. Eur J Cancer 2007;43:1348-60.

114. Duffy MJ, Lamerz R, Haglund C, Nicolini A, Kalousová M, Holubec L, Sturgeon C. Tumor markers in colorectal cancer, gastric cancer and gastrointestinal stromal cancers: European group on tumor markers 2014 guidelines update. Int J Cancer 2014;134:2513-22..

115. Kanellos I, Zacharakis E, Demetriades H, Christoforidis E, Kanellos D, Pramateftakis MG, Betsis D. Value of carcinoembryonic antigen assay in predicting hepatic metastases, local recurrence, and survival after curative resection of colorectal cancer. Surg Today 2006;36: 879-84.

116. Yoon HI, Koom WS, Kim YB, Min BS, Lee KY, Kim NK, et al. Predicting the pathologic response of locally advanced rectal cancer to neoadjuvant concurrent chemoradiation using enzyme-linked immunosorbent assays (elisas) for biomarkers. J Cancer Res Clin Oncol 2014;140:399-409.

117. Johnson DA, Barclay RL, Mergener K, Weiss G, König T, Beck J, Potter NT. Plasma septin9 versus fecal immunochemical testing for colorectal cancer screening: A prospective multicenter study. PLoS One 2014;9:e98238.

118. Church TR, Wandell M, Lofton-Day C, Mongin SJ, Burger M, Payne SR, et al. Prospective evaluation of methylated SEPT9 in plasma for detection of asymptomatic colorectal cancer. Gut 2014;63:317-25.

119. Pratt VM. Are we ready for a blood-based test to detect colon cancer? Clin Chem 2014;60:1141-2.

120. Ebert MP, Tänzer M, Balluff B, Burgermeister E, Kretzschmar AK, Hughes DJ, et al. TFAP2EDKK4 and chemoresistance in colorectal cancer. N Engl J Med 2012;366:44-53.

121. Penning $C$. Landelijke monitoring van het bevolkingsonderzoek darmkanker: Resultaten eerste halfjaar 2014. 2014, Oct 6 .

122. Nishihara R, Wu K, Lochhead P, Morikawa T, Liao X, Qian ZR, et al. Long-term colorectalcancer incidence and mortality after lower endoscopy. N Engl J Med 2013, Sep 19;369(12):1095-105.

123. Heleno B, Thomsen MF, Rodrigues DS, Jørgensen KJ, Brodersen J. Quantification of harms in cancer screening trials: Literature review. BMJ 2013;347:f5334.

124. Ignatiadis M, Dawson SJ. Circulating tumor cells and circulating tumor DNA for precision medicine: Dream or reality? Ann Oncol 2014;25:2304-2313.. 
125. De Mattos-Arruda L, Weigelt B, Cortes J, Won HH, Ng CK, Nuciforo P, et al. Capturing intratumor genetic heterogeneity by de novo mutation profiling of circulating cell-free tumor DNA: A proof-of-principle. Ann Oncol 2014;25:1729-35.

126. Mohan S, Heitzer E, Ulz P, Lafer I, Lax S, Auer M, et al. Changes in colorectal carcinoma genomes under anti-egfr therapy identified by whole-genome plasma DNA sequencing. PLoS Genet 2014;10:e1004271.

127. Heitzer E, Auer M, Ulz P, Geigl JB, Speicher MR. Circulating tumor cells and DNA as liquid biopsies. Genome Med 2013;5:73.

128. Heitzer E, Ulz P, Belic J, Gutschi S, Quehenberger F, Fischereder K, et al. Tumor-associated copy number changes in the circulation of patients with prostate cancer identified through whole-genome sequencing. Genome Med 2013;5:30.

129. Barbazán J, Muinelo-Romay L, Vieito M, Candamio S, Díaz-López A, Cano A, et al. A multimarker panel for circulating tumor cells detection predicts patient outcome and therapy response in metastatic colorectal cancer. Int J Cancer 2014;135:2633-43.

130. Alix-Panabieres C, Pantel K. [The circulating tumor cells: Liquid biopsy of cancer]. Klin Lab Diagn 2014, Apr(4):60-4.

131. Herwig R, Mitteregger D, Djavan B, Kramer G, Margreiter M, Leers MP, et al. Detecting prostate cancer by intracellular macrophage prostate-specific antigen (PSA): A more specific and sensitive marker than conventional serum total PSA. European Journal of Clinical Investigation 2008;38:430-7.

132. Japink D, Leers MPG, Sosef MN, Nap M. CEA in activated macrophages. New diagnostic possibilities for tumor markers in early colorectal cancer. Anticancer Res 2009;29:3245.

133. Sturgeon. Quality requirements and control: EGTM recommendations. European group on tumour markers. Anticancer Res 1999;19:2791-4.

134. Diamandis EP. Tumor markers : Physiology, pathobiology, technology, and clinical applications. illustrated ed. Washington, DC: Amer. Assoc. for Clinical Chemistry; 2002.

135. Dot D, Miró J, Fuentes-Arderiu X. Biological variation of the leukocyte differential count quantities. Scand J Clin Lab Invest 1992;52:607-11.

136. Sturgeon CM, Duffy MJ, Stenman UH, Lilja H, Brünner N, Chan DW, et al. National academy of clinical biochemistry laboratory medicine practice guidelines for use of tumor markers in testicular, prostate, colorectal, breast, and ovarian cancers. Clin Chem 2008;54:e11-79.

137. Herwig R, Mitteregger D, Djavan B, Kramer G, Margreiter M, Leers MP, et al. Detecting prostate cancer by intracellular macrophage prostate-specific antigen (PSA): A more specific and sensitive marker than conventional serum total PSA. European Journal of Clinical Investigation 2008;38:430-7.

138. Japink D, von Meyenfeldt MF, Nap M, Sosef MN, Verheyen F, Beets GL, Leers MPG. Visualizing CEA in activated macrophages using electron microscopy. (Submitted). 2014, Jun 1.

139. Faber TJ, Japink D, Leers MP, Sosef MN, von Meyenfeldt MF, Nap M. Activated macrophages containing tumor marker in colon carcinoma: Immunohistochemical proof of a concept. Tumour Biol 2011;33:435-41.

140. Japink D, Nap M, Sosef MN, Nelemans PJ, Coy JF, Beets G, et al. Reproducibility studies for experimental epitope detection in macrophages (EDIM). J Immunol Methods 2014;407:40-7.

141. Fertl A, Menzel M, Hofer TP, Morresi-Hauf A, Ziegler-Heitbrock L, Frankenberger M. Monitoring of glucocorticoid therapy by assessment of CD14(+)CD16(+) monocytes: A case report. Immunobiology 2008;213:909-16.

142. Horelt A, Belge KU, Steppich B, Prinz J, Ziegler-Heitbrock L. The CD14+CD16+ monocytes in erysipelas are expanded and show reduced cytokine production. Eur J Immunol 2002;32: 1319-27.

143. Tudyka V, Blomqvist L, Beets-Tan RG, Boelens PG, Valentini V, van de Velde CJ, et al. EURECCA consensus conference highlights about colon \& rectal cancer multidisciplinary management: The radiology experts review. Eur J Surg Oncol 2014;40:469-75. 
144. Buijsen J, van Stiphout RG, Menheere PP, Lammering G, Lambin P. Blood biomarkers are helpful in the prediction of response to chemoradiation in rectal cancer: A prospective, hypothesis driven study on patients with locally advanced rectal cancer. Radiother Oncol 2014;111:237-42..

145. Herwig R, Djavan B, Kramer G, El-Taieb MA, Kuhhas F, Leers M, Marberger M. [Prostate cancer screening with a new marker based on circulating blood macrophages?]. Urologe $A$ 2007;46:1066-70.

146. Sturgeon CM. Tumor markers in the laboratory: Closing the guideline-practice gap. Clin Biochem 2001;34:353-9.

147. Maas M, Beets-Tan RG, Lambregts DM, Lammering G, Nelemans PJ, Engelen SM, et al. Waitand-see policy for clinical complete responders after chemoradiation for rectal cancer. J Clin Oncol 2011;29:4633-40.

148. Lambregts DM, Rao SX, Sassen S, Martens MH, Heijnen LA, Buijsen J, et al. MRI and diffusion-weighted MRI volumetry for identification of complete tumor responders after preoperative chemoradiotherapy in patients with rectal cancer: A bi-institutional validation study. Ann Surg 2014, Sep 10.

149. Janssen MH, Öllers MC, van Stiphout RG, Riedl RG, van den Bogaard J, Buijsen J, et al. PETbased treatment response evaluation in rectal cancer: Prediction and validation. Int $\mathrm{J}$ Radiat Oncol Biol Phys 2012;82:871-6.

150. Janssen MH, Ollers MC, Riedl RG, van den Bogaard J, Buijsen J, van Stiphout RG, et al. Accurate prediction of pathological rectal tumor response after two weeks of preoperative radiochemotherapy using (18)f-fluorodeoxyglucose-positron emission tomography-computed tomography imaging. Int J Radiat Oncol Biol Phys 2010;77:392-9.

151. van Stiphout RG, Lammering G, Buijsen J, Janssen MH, Gambacorta MA, Slagmolen P, et al. Development and external validation of a predictive model for pathological complete response of rectal cancer patients including sequential PET-CT imaging. Radiother Oncol 2011;98: 126-33. 



\title{
CHAPTER 2
}

Agtifated macrophages containing tumour marker in colon carcinoma: immunohistochemical proof of a concept

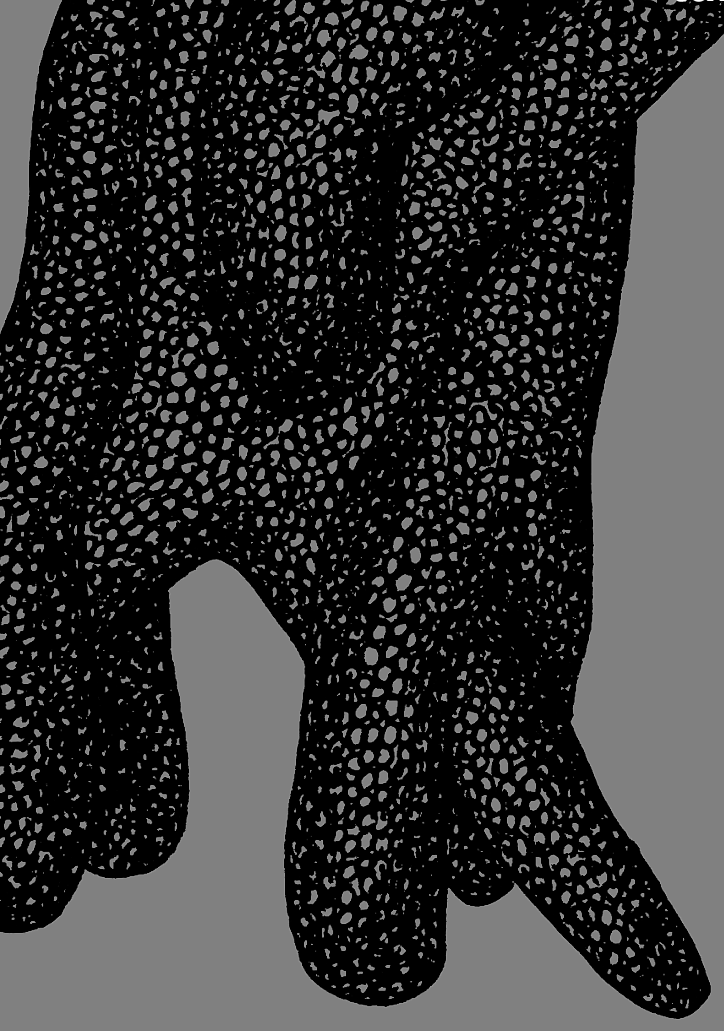

\author{
Tjitske JE Faber \\ Dennis Japink \\ Mathie PG Leers \\ Meindert N Sosef \\ Maarten F von Meyenfeldt \\ Marius Nap
} Tumour Biol 2012;33:435-441 


\section{Abstract}

\section{Introduction}

The presence of carcinoembryonic antigen (CEA)-containing activated macrophages has been demonstrated in peripheral blood from patients with colorectal carcinoma. Macrophages migrate from the circulation into tissue, phagocytose debris and return to the bloodstream. Hence it seems likely that activated macrophages containing tumour debris i.e. tumour marker are present in the stroma of colorectal carcinoma. After phagocytosis, they could follow a haematogenic or lymphogenic route to peripheral blood.

\section{Aims}

To assess the presence of tumour marker containing activated macrophages in the stroma of colon carcinoma and in regional lymph nodes.

\section{Methods}

From 10 cases of colon carcinoma, samples of tumour tissue and metastasis-free lymph nodes were cut in serial sections and stained for CD68 to identify macrophages and for CEA-, cytokeratin- or M30-presence. Slides were digitalised and visually inspected using two monitors, comparing the CD68 stain to the tumour marker stain to evaluate the presence of tumour marker positive macrophages.

\section{Results}

Macrophages containing tumour marker could be identified in tumour stroma and in metastasis-free regional lymph nodes. The distribution varied for the different markers, CEA-positive macrophages being most abundant.

\section{Conclusions}

The presence of macrophages containing tumour marker in tumour stroma and lymph nodes from patients with colon carcinoma could be confirmed in this series using serial immunohistochemistry. This finding supports the concept of activated macrophages, after phagocytosing cell debris, being transported or migrating through the lymphatic system. These results support the potential of tumour marker containing macrophages to serve as a marker for diagnosis and follow-up of colon cancer patients. 


\section{Introduction}

Activated (CD14+/CD16+) macrophages containing carcinoembryonic antigen (CEA) and prostate-specific antigen (PSA) have been identified in peripheral blood from respectively colorectal and prostate cancer patients, using flow cytometry $^{1,2}$. In melanoma-associated macrophages, melanin-containing melanosomes have been described, both at the tumour site as well as in regional lymph nodes ${ }^{3,4}$. The CD14+/CD16+ subpopulation of monocytes resembles tissue macrophages in many ways ${ }^{5}$. Activated blood-borne monocytes/ macrophages are able to migrate into tissues and replenish tissuespecific macrophages, phagocytosing substances (e.g. cell debris) at the site in both tumour environment as well as in non-neoplastic conditions ${ }^{6-9}$.

This pilot study intends to evaluate the concept of tumour marker containing macrophages in colon carcinoma tissue and regional metastasis-free lymph nodes, using double monitor comparison of immunohistochemistry on serial sections to visualise macrophages and tumour markers CEA, cytokeratin and M30.

\section{Materials and methods}

\subsection{Patient and sample selection}

For this pilot study, ten patients recently operated for colon carcinoma (confirmed on histopathological evaluation) were included. Age, tumour localisation, tumour size and tumour stage according to the American Joint Committee on Cancer (AJCC) were retrieved from medical records. For each case, original histopathological reports and $\mathrm{H} \& \mathrm{E}$ stained sections were reviewed and the carcinoma sample showing most invasive growth was selected. In one case, the initial diagnostic biopsy was selected for analysis because it showed more invasive growth than the tumour in the colon resection specimen. In one case two separate tumours were found in one resection; we chose to evaluate the more invasive tumour. For each tumour sample, from the same surgical specimen 2 regional lymph nodes free of metastasis were selected. All selected samples were evaluated for presence of tumour marker containing macrophages. 


\subsection{Immunohistochemistry}

All paraffin-embedded colon carcinoma samples and lymph node samples were serially sectioned in four consecutive $3 \mu \mathrm{m}$ slides. Sections were deparaffinised with xylene and rehydrated through graded ethanol. For each sample sections were stained with anti-CD-68, anti-CEA, anti-cytokeratin and anti-M30 antibodies respectively.

Antigen retrieval was performed in different ways for the different antigens. For CD68 and M30, specimens were placed in $0,01 \mathrm{M}$ citrate buffer at $\mathrm{pH} 6.0$, incubated for 20 minutes at $98^{\circ} \mathrm{C}$ and allowed to cool in buffer. For CEA, specimens were placed in EDTA buffer at $\mathrm{pH} 9.0$, incubated for 20 minutes at $98^{\circ} \mathrm{C}$ and allowed to cool in buffer. For cytokeratin, specimens were incubated with $2 \mathrm{ml}$ pepsin $1 \mathrm{mg} / \mathrm{ml}$ in $0,1 \mathrm{M} \mathrm{HCl}$ at $37^{\circ} \mathrm{C}$ for 15 minutes, then cooled and rinsed in tap water.

Next, slides were incubated with Dako Dual Endogenous Enzyme Block (Dako) for 5 minutes to block endogenous peroxidase.

These steps were followed by incubation with primary antibodies. For detection of macrophages, anti-CD-68 (clone PG-M1, Dako Cytomation, Denmark) commonly used as a marker of monocyte-macrophage lineage cells ${ }^{10-12}$, was used. To visualise the presence of CEA, clone II-7 was used. This clone is monospecific for CEA ${ }^{13}$. Mouse monoclonal anti-cytokeratin (clone MNF 116, Dako) was used to visualise cytokeratins, commonly found in the cytoskeleton of mammalian cells and many carcinomas. Anti-M30 Cytodeath (clone M30, Peviva, Sweden) was used to visualise $\mathrm{M} 30$, an antibody that recognises a neoepitope of cytokeratin 18 exposed after caspase-mediated cleavage during early apoptosis (14). Antibodies were diluted in Dako Chem Mate Antibody Diluent (Dako). Antibody dilutions used were 1:100 for anti-CD68 and anti-M30, and 1:200 for anti-CEA and anti-cytokeratin.

Antibody binding was visualized using the Dako Autostainer by means of the Dako EnVision Detection System, Peroxidase/diaminobenzidine (DAB, Dako) kit for CD68, CEA and cytokeratin and aminoethylcarbazole (AEC Single Solution, Zymed, USA) for M30. Slides were incubated with Envision HRP rabbit/mouse solution (Dako) for 30 minutes and were incubated with DAB (CD68-, CEA-, and cytokeratin-staining) or AEC (M30-staining) for 5 minutes twice, yielding a respectively brown or red substrate at the site of the target antigen. Finally, all slides were counterstained with haematoxylin and coverslipped. 


\subsection{Scoring of immunohistochemistry}

Slides created were digitalised using the 3DH/Zeiss Automatic Brightfield Scan microscopy scanner (Zeiss, Germany) and viewed in MIRAX Viewer (3DHISTECH, Hungary) version 1.12.22.1.

Digital slides were evaluated using a double-screen setup, allowing for simultaneous viewing of the CD68 stain on one screen and one of the tumour marker stains from the same case on the other screen. Identical areas were identified and here we searched for morphologically macrophage-like cells that stained positively on the CD68 stain and also on the tumour marker stain, illustrated in Figure 2.1. Tumour marker containing macrophages were digitally annotated. Annotations were made in different colors for the respective tumour markers. All slides were evaluated by one observer. In case of doubt a second independent observer assessed the unresolved cases. If interpretation still remained unclear, the original section was reviewed in conventional microscopy. Ambiguous structures were not marked as positive.

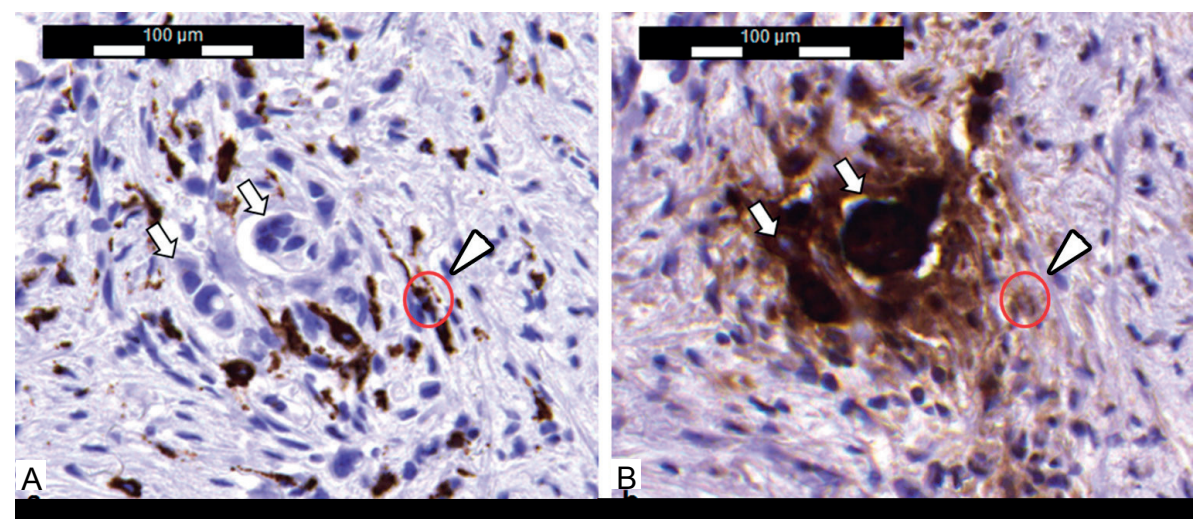

Figure 2.1 A. CD68 stain of tumour sample; B. corresponding CEA stain of the same sample. Tumour cell clusters are visible (arrows) with diffuse staining around tumour cells. An example of a CEA containing macrophage with granular staining can be seen to the right of the tumour cells (circle and arrowhead); the CD68 stain shows the presence of macrophages around the tumour and diffusely in the tumour stroma whereas the tumour cell cluster is negative 


\subsection{Analysis}

Primary tumour: the area with the highest density of tumour marker positive macrophages was identified. In this area, the number of tumour marker positive macrophages per 2 square $\mathrm{mm}$ was counted. For each tumour stage and the total group, medians with ranges were determined.

Lymph nodes: the surface area of every lymph node was measured and the number of tumour marker positive macrophages per 2 square $\mathrm{mm}$ was recorded for each lymph node. For each tumour stage and the whole group, medians with ranges were determined.

\section{Results}

\subsection{Baseline characteristics}

From 10 patients 10 tumours and 20 lymph nodes were analysed. The median age of patients included in this study was 74 years (range 57-90 years). The male:female ratio was 1:1. Three AJCC stage I tumours were included, one stage II tumour and six stage III tumours. No stage IV tumours were included in the study. Patient and tumour characteristics are summarised in Table 2.1.

$\begin{array}{ll}\text { Table 2.1 } & \text { Patient and tumour characteristics }(\mathrm{n}=10) \\ \text { Gender } & \mathrm{n}= \\ \text { male } & 5(50 \%) \\ \text { Age } & \text { Median in years (range) } \\ \text { total } & 73(57-90) \\ \text { male } & 68(57-82) \\ \text { female } & 78(61-90) \\ \text { Tumour stage (AJCC) } & \mathrm{n}= \\ \text { I (T1-2, N0) } & 3 \\ \text { II (T3-4, N0) } & 1 \\ \text { III (any N+) } & 6 \\ \text { IV (any M+) } & 0\end{array}$

\subsection{Digital images}

The setup with two monitors, displaying the CD68 and a tumour marker stain facilitated the examination of the different staining patterns in the same area. By annotating the identified macrophages with different colours for each marker on the CD68 image, differences or similarities in distribution of different markers could readily be identified. 


\subsection{Tumour stroma}

In 10/10 cases, activated macrophages positive for CEA, cytokeratin and M30 could be identified. Results are summarized in Table 2.2.

Table 2.2 Median number of tumour marker positive macrophages in tumour stroma per 2 square $\mathrm{mm}$ in the area of highest density grouped by AJCC stage, with ranges.

$\begin{array}{lccc}\text { Tumour stage (AJCC) } & \text { M30 } & \text { Cytokeratin } & \text { CEA } \\ \text { I }(n=3) & 3(1-9) & 11(4-21) & 362(93-498) \\ \text { II }(n=1) & 2 & 13 & 265 \\ \text { III }(n=6) & 3(2-10) & 10(4-14) & 100(4-344) \\ \text { All }(n=10) \text { and range } & 3(1-10) & 11(4-21) & 199(4-498)\end{array}$

CD68-positive macrophages were encountered in different places. The highest concentrations were found in desmoplastic stroma in the periphery of the tumour. Macrophages were also found directly adjacent to tumour glands, in the lumina of tumour glands, in blood vessels and in lymphatic vessels. To a lesser extent, macrophages were found in the lamina propria and submucosa of the adjacent normal colon.

A substantial number of activated macrophages showing positivity for CEA could be identified. CEA-positive macrophages were found in all abovementioned localisations of the macrophages. One slide showed a CEA-positive macrophage in a blood vessel wall, suggesting migration of macrophages after phagocytosis (Figure 2.2). Most positive activated macrophages showed intracellular deposits of CEA (Figure 2.1). In a number of tumours, we observed diffusion of the staining directly adjacent to the tumour epithelium, in an area with a high density of activated macrophages (Figure 2.1). These macrophages were considered positive. Two stage III tumours were found to be partially CEAnegative but still contained a number of CEA-positive macrophages.

Cytokeratin positive macrophages were present in smaller numbers than CEApositive macrophages (Table 2.2) and in the serial sections; they were not present at the same location.

M30 tumour cell positivity was found in all tumours, especially within the lumina of tumour glands and in areas of apoptosis. M30 positive activated macrophages were sporadically found in tumour stroma. Since this study assessed properties of tissue-associated macrophages, only these cells were evaluated. The number of macrophages positive for M30 was substantially lower than for CEA and cytokeratin (Table 2.2). 
Although this study does not allow any definitive conclusions, the results shown in Table 2.2 suggest that for higher tumour stages, the number of tumour-marker containing macrophages in tumour stroma decreases.
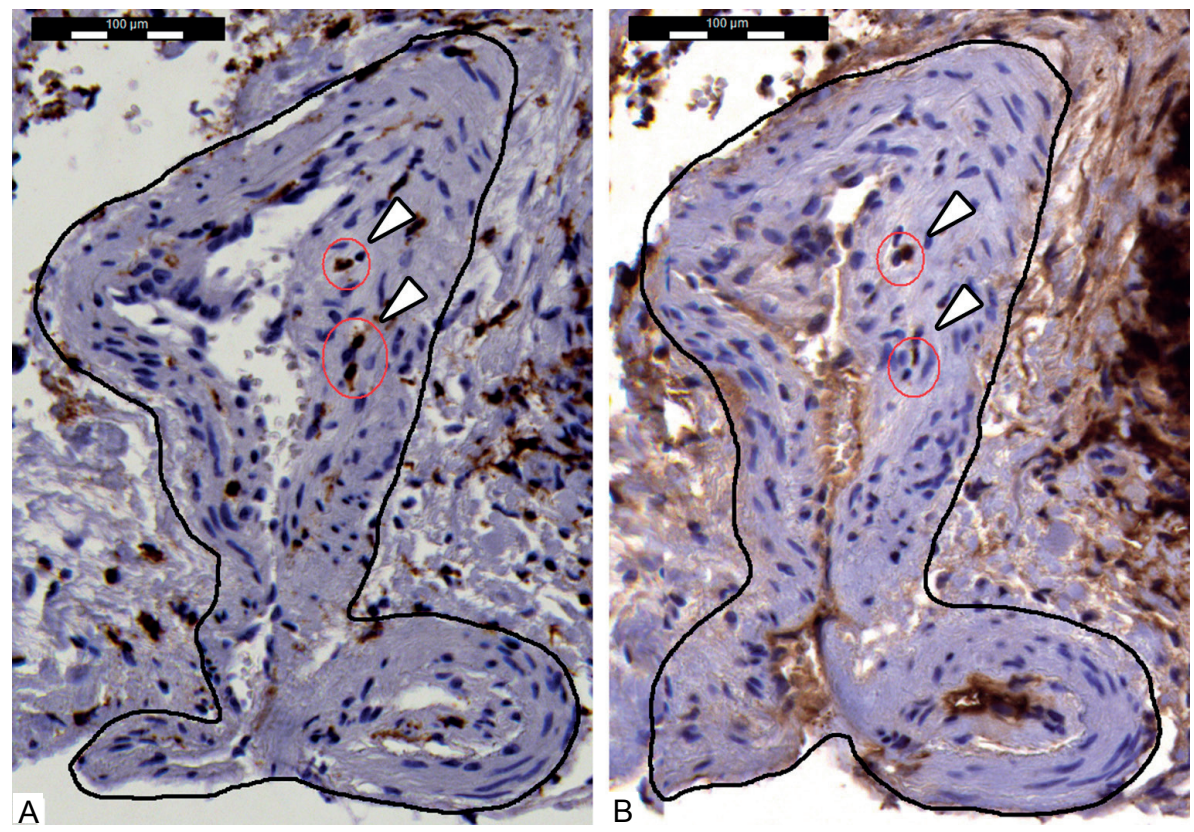

Figure 2.2 A. CD68 stain of a blood vessel (outlined) next to tumour tissue; B. CEA stain of the same blood vessel. Intramural macrophages are present; two macrophages are positive in the CEA stain (arrowheads, circles). CEA reactivity is also visible at the luminal borders of this vessel.

\subsection{Lymph nodes}

We evaluated 20 lymph nodes from the same 10 patients. In 19/20 lymph nodes, macrophages containing tumour marker could be identified. In one case, M30 containing macrophages could not be identified. Results are summarized in Table 2.3.

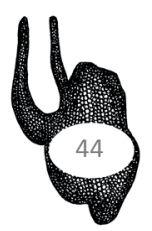


Table 2.3 Median number of tumour marker positive macrophages in lymph nodes per 2 square $\mathrm{mm}$ grouped by AJCC stage, with ranges.

$\begin{array}{lccc}\text { Tumour stage (AJCC) } & \text { M30 } & \text { Cytokeratin } & \text { CEA } \\ \text { I }(n=6) & 0,7(0,2-1,0) & 3,1(0,3-5,7) & 9,5(1,4-65,7) \\ \text { II }(n=2) & 0,6(0,5-0,7) & 5,7(2,6-5,7) & 1,4(1,4-1,4) \\ \text { III }(n=12) & 0,8(0,0-5,8) & 1,4(0,6-5,9) & 3,3(0,6-53,9) \\ \text { All }(n=20) \text { and range } & 0,8(0,0-5,8) & 2,3(0,3-8,7) & 4,3(0,6-65,7)\end{array}$

CD68-positive macrophages were found along the lining of the medullary sinuses and in lymphatic nodules. In smaller amounts, macrophages were found in cortical sinuses, medullary chords and within the cortical parenchyma.

Similar to our findings within the tumour stroma, CEA containing macrophages were found in relatively large numbers when compared to M30 and cytokeratin. Macrophages with CEA positivity showed a characteristic granular cytoplasmic staining and were almost exclusively found along the lining of the medullary sinuses (Figure 2.3).
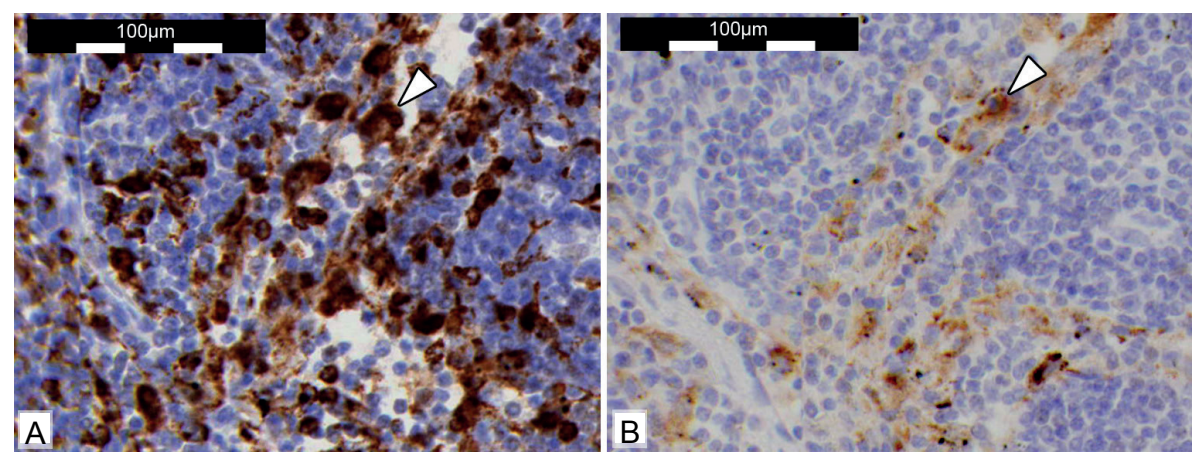

Figure 2.3 A. CD68 stain of a lymph node with widespread macrophage distribution; B. corresponding CEA stain. CEA-containing macrophages can be seen along the lining of and are limited to the medullary sinuses. Granular staining is visible in a number of macrophages (example at arrow)

Cytokeratin containing macrophages, though fewer in number, showed a less restrictive distribution. These macrophages were found within the sinuses, and in the subcapsulary sinuses, medullary chords and cortex parenchyma as well. Within lymphatic nodules, cytokeratin containing macrophages were only sporadically seen. Some perivascular elements showed cytokeratin positivity but 
could be differentiated from macrophages based on morphological characteristics and a lack of corresponding CD68 staining.

M30 containing macrophages were found in similar locations as cytokeratincontaining macrophages. In contrast to cytokeratin and CEA, these macrophages were also found in lymphatic nodules. M30 positive macrophages were found only sporadically compared to CEA- and cytokeratin-positive macrophages (Table 2.3).

\section{Discussion}

To the best of our knowledge this is the first report on the possible route of transportation followed by tumour associated fragments from the tumour site to the blood stream, supported by morphological and immunohistochemical observations. We propose that circulating tumour marker containing CD14+/CD16+ macrophages ${ }^{1,2}$ may have ingested this material at the tumour site.

The exact route macrophages follow after phagocytosing tumour material is unclear. In a mouse model of retinal injury, Joly et al demonstrated the migration of blood borne macrophages into an area of injury. Microglia, the retinal resident tissue macrophages, showed trafficking abilities towards the injured retinal side, and adopted signs of phagocytosis. In reaction to the injury, both at the site of injury and healthy tissue, the number of macrophages rose by influx via blood vessels, adding extra phagocytic capacity to the injured site. Macrophages loaded with debris attaching to and crossing into capillaries suggest a route back into the circulation ${ }^{15}$. Their findings also give support to the hypothesis that tissue associated macrophages can engulf tumour particles and transport them to vascular structures.

Blood-borne activated macrophages containing tumour material as found in earlier studies ${ }^{1,2}$ have likely obtained tumour material while residing in stromal tissue at the tumour site and afterwards migrated towards the circulation. In this series, macrophages were found mostly at the margin of the tumour and also in the tumour stroma, consistent with earlier findings ${ }^{16}$. We identified a tumour marker containing macrophage in a blood vessel wall in one section, supporting the hypothesis of haematogenic migration. According to anatomical venous outflow, cells entering blood vessels will travel through the portal vein towards the liver. Examining (peroperative) portal blood and liver tissue from patients 
with colon carcinoma could provide more insight into the mechanisms involved in this process.

However, from the assessment of regional lymph nodes we can conclude that TM-containing macrophages also travel through the lymphatic system towards regional lymph nodes. In case reports of malignant melanoma, melanincontaining macrophages have been demonstrated in sentinel lymph nodes, even without the presence of tumour in the lymph nodes ${ }^{4,17}$. TM-containing macrophages were more readily identified in lymph nodes than in blood vessels in this study. This may suggest a preference for these cells to travel via lymphogenic route. Although both a lymphogenic and haematogenic route are possible travel itineraries for TM-containing macrophages our results seem to concur with the observation of metastases being present in lymph nodes more frequent than in organs reached through a haematogenic route such as liver, lungs or the skeletal system.

Macrophages are versatile cells that can exhibit different functional phenotypes. Macrophages have been divided into the M1 and M2 type. M1 macrophage activity includes pro-inflammatory functions, including the capacity to kill microorganisms and the production of proinflammatory cytokines. M2 macrophages are involved in immunosuppression, tissue remodelling, angiogenesis and scavenging of debris, including apoptotic cells ${ }^{18,19}$. The division in $M 1$ and $M 2$ type macrophages may not be absolute ${ }^{20}$, enabling macrophages to switch from one function type to another or assuming characteristics of more than one type at a time. Tumour-associated macrophages (TAM) may play an important role in tumour progression, angiogenesis, metastatic ability and remodelling of the extracellular matrix ${ }^{21}$.

Wyckoff et al. ${ }^{16}$ found that macrophages only phagocytosed labelled sugars while in the stroma of a tumour, as opposed to while circulating in the blood. This may indicate a phenotype difference between blood borne and tissueassociated macrophages, possibly under influence of cytokines and (tumourderived) growth factors ${ }^{22-24}$. Nuclear condensation as seen in blood-borne tumour marker containing macrophages ${ }^{1}$ could indicate macrophages may have reached the end of their life cycle and initiate apoptosis after fulfilling their role in tumour stroma. A vitality stain could demonstrate whether blood-borne macrophages containing tumour material are indeed in the process of apoptosis. Tumour marker containing in macrophages showed a typical granular staining pattern. In 2010, Lazova et al. ${ }^{3}$ described melanoma-associated macrophages 
containing coarse melanin in a similar staining pattern. Electron microscopy and immunohistochemistry showed that these melanosomes were contained in autophagosomes and/or autolysosomes in the macrophage cytoplasm. This may also be the case with CEA in colon carcinoma-associated macrophages.

Pawelek et al. ${ }^{25}$ have postulated the fusion of tumour cells with bone marrow derived cells as a possible explanation for tumour progression and metastasis. Such hybrid cells have been identified in vitro and in sporadic cases in vivo. The presence of tumour marker in macrophages could be a characteristic of tumourmacrophage hybrid cells. Electron microscopy may be of use to further evaluate the interaction between macrophages and tumour material.

We found substantial differences between the various tumour markers in the densities of tumour marker containing macrophages. This gives rise to a number of hypotheses. Firstly, there may be a relation between the presence and distribution of the investigated substances in the tumour and the possibility to find loaded macrophages. Rupa et al described the amount of M30-positive cells in colorectal adenocarcinomas to be $5-17 \%$, with a mean of $11,1 \%{ }^{26}$, while almost all colorectal carcinoma epithelial cells exhibit cytokeratin and CEA positivity. Therefore, the argument of varying presence of tumour marker might be true for CEA compared to M30 but is not likely to be true for CEA compared to cytokeratins. Secondly, the stability of the substance may play a role. CEA is an extremely stable molecule that can even survive perchloric extraction, while cytokeratins are easily damaged by electrical or chemical agents. Thirdly it might be that the high level of glycosylation of CEA facilitates the interaction with tissue macrophages compared to cytokeratins. Fourthly, a macrophage does not only phagocytose substances. Phagosomes fuse with lysosomes containing various enzymes, which may degrade antigens, making them unrecognisable to our antibodies. A combination of all abovementioned mechanisms - availability, selective phagocytosis, molecule stability and degradation - is also possible.

Although we did not apply double staining procedures, the serial section approach consistently showed different areas of distribution for macrophages with the three antigens investigated in this pilot study. This was observed both in the tumour stroma but even more prominent in the lymph nodes. Cross-reaction with the non-specific cross-reacting antigen (NCA), a macrophage component, might make interpretation more difficult; however, clone II-7 was one of the 
highly CEA specific antibodies in a comparative study on multiple CEA antibodies $^{13}$. Also, the fact that CEA positive macrophages were not found in all lymph nodes and their distribution in the lymph node was limited to a subset of cells gave further support to a meaningful interpretation of this observation.

The use of double chromogenic stains does not seem to be an alternative, because we attempt to stain different structures both located in the cytoplasm of a single cell. Using direct fluorochrome labelled antibodies and confocal laser scanning microscopy may be a better option to answer the question of colocalisation of different substances in the same macrophages. Considering the typical granular staining of intra-macrophage tumour marker, the observed anatomical distribution of tumour markers and macrophages, and morphological characteristics of macrophages, serial section immunohistochemistry seems adequate for this pilot experiment evaluating whether or not tumour marker containing macrophages are present in colorectal tumour stroma and lymph nodes.

\section{Conclusion}

In this study we were able to identify tissue-associated macrophages both in tumours and in their lymphatic draining area. Serial sectioning and immunohistochemistry is a relatively simple method to identify tumour marker containing macrophages.

Macrophages containing tumour material are present in the stroma of colon carcinoma and in regional metastasis-free lymph nodes. The number of tumour marker positive macrophages varied between cases as well as between the different tumour markers. The distribution of tumour marker positive macrophages is different for the various markers used in this study. 


\section{References}

1. Japink D, Leers MPG, Sosef MN, Nap M. CEA in activated macrophages. New diagnostic possibilities for tumor markers in early colorectal cancer. Anticancer Res 2009;29: 3245.

2. Leers MPG, Nap M, Herwig R, Delaere K, Nauwelaers F. Circulating psa-containing macrophages as a possible target for the detection of prostate cancer: A three-color/fiveparameter flow cytometric study on peripheral blood samples. Am J Clin Pathol. 2008;129:649.

3. Lazova R, Klump V, Pawelek J. Autophagy in cutaneous malignant melanoma. J Cutan Pathol 2010;37:256-68.

4. Malafronte $\mathrm{P}$, Sorrells $\mathrm{T}$. Lymph node melanosis in a patient with metastatic melanoma of unknown primary. Arch Pathol Lab Med 2009;133:1332-4.

5. Ziegler-Heitbrock L. The CD14+ CD16+ blood monocytes: their role in infection and inflammation. J Leukoc Biol 2007;81:584-92.

6. Yuan A, Chen JJ, Yang PC. Pathophysiology of tumor-associated macrophages. Adv Clin Chem 2008;45:199.

7. Sunderkötter C, Nikolic T, Dillon MJ, Van Rooijen N, Stehling M, Drevets DA, Leenen PJ. Subpopulations of mouse blood monocytes differ in maturation stage and inflammatory response. J Immunol 2004;172:4410-7.

8. Geissmann F, Jung S, Littman DR. Blood monocytes consist of two principal subsets with distinct migratory properties. Immunity 2003;19:71-82.

9. Hume DA, Ross IL, Himes SR, Sasmono RT, Wells CA, Ravasi T. The mononuclear phagocyte system revisited. J Leukoc Biol 2002;72:621-7.

10. Nomura Y, Takeuchi M, Yoshida S, Sugita Y, Niino D, Kimura Y, Shimizu K, Aoki R, Suefuji N, Hirose S, Kikuchi M, Ohshima K. Phenotype for activated tissue macrophages in histiocytic necrotizing lymphadenitis. Pathol Int 2009;59:631-5.

11. Gottfried E, Kunz-Schughart LA, Weber A, Rehli M, Peuker A, Müller A, Kastenberger M, Brockhoff G, Andreesen R, Kreutz M. Expression of CD68 in non-myeloid cell types. Scand J Immunol 2008;67:453-63.

12. Kunisch E, Fuhrmann R, Roth A, Winter R, Lungershausen W, Kinne RW. Macrophage specificity of three anti-CD68 monoclonal antibodies (KP1, EBM11, and PGM1) widely used for immunohistochemistry and flow cytometry. Ann Rheum Dis 2004;63:774-84.

13. Nap M, Hammarström ML, Börmer O, Hammarström S, Wagener C, Handt S, Schreyer M, Mach JP, Buchegger F, von Kleist S, et al. Specificity and affinity of monoclonal antibodies against carcinoembryonic antigen. Cancer Res 1992;52:2329-39.

14. Leers MP, Kölgen W, Björklund V, Bergman T, Tribbick G, Persson B, Björklund P, Ramaekers FC, Björklund B, Nap M, Jörnvall H, Schutte B. Immunocytochemical detection and mapping of a cytokeratin 18 neo-epitope exposed during early apoptosis. The Journal of Pathology. 1999;187:567-72.

15. Joly $S$, Francke $M$, Ulbricht $E$, Beck $S$, Seeliger $M$, Hirrlinger $P$, Hirllinger J, Lang KS, Zinkernagel M, Odermatt B, Samardzija M, Reichenbach A, Grimm C, Remé CE. Cooperative Phagocytes: Resident Microglia and Bone Marrow Immigrants Remove Dead Photoreceptors in Retinal Lesions. Am J Pathol 2009;174:2310-23.

16. Wyckoff JB, Wang Y, Lin EY, Li JF, Goswami S, Stanley ER, Segall JE, Pollard JW, Condeelis $\mathrm{J}$. Direct visualization of macrophage-assisted tumor cell intravasation in mammary tumors. Cancer Res 2007;67:2649-56.

17. Satzger I, Völker B, Kapp A, Gutzmer R. Tumoral melanosis involving the sentinel lymph nodes: a case report. Journal of Cutaneous Pathology 2007;34:284-6.

18. Mantovani A, Sozzani S, Locati M, Allavena P, Sica A. Macrophage polarization: tumorassociated macrophages as a paradigm for polarized M2 mononuclear phagocytes. Trends Immunol 2002;23:549-55.

19. Mantovani A, Sica A, Sozzani S, Allavena P, Vecchi A, Locati M. The chemokine system in diverse forms of macrophage activation and polarization. Trends Immunol 2004;25:677-86. 
20. Mosser DM, Edwards JP. Exploring the full spectrum of macrophage activation. Nat Rev Immunol. 2008;8(12):958-69.

21. Mueller MM, Fusenig NE. Friends or foes - bipolar effects of the tumour stroma in cancer. Nat Rev Cancer. 2004;4(11):839-49.

22. Sica A, Allavena $P$, Mantovani A. Cancer related inflammation: The macrophage connection. Cancer Letters. 2008;267:204-15.

23. Bingle L, Brown NJ, Lewis CE. The role of tumour-associated macrophages in tumour progression: implications for new anticancer therapies. J Pathol 2002;196:254-65.

24. Pollard JW. Macrophages define the invasive microenvironment in breast cancer. J Leukoc Biol. 2008 September 1, 2008;84:623-30.

25. Pawelek JM, Chakraborty AK. Fusion of tumour cells with bone marrow-derived cells: a unifying explanation for metastasis. Nat Rev Cancer. 2008;8:377-86.

26. Rupa JD, de Bruïne AP, Gerbers AJ, Leers MPG, Nap M, Kessels AGH, et al. Simultaneous detection of apoptosis and proliferation in colorectal carcinoma by multiparameter flow cytometry allows separation of high and low-turnover tumors with distinct clinical outcome. Cancer. 2003;97:2404-11. 



\section{Abstract}

\section{Introduction}

Carcinoembryonic Antigen (CEA) can be detected in activated macrophages (AM) in colorectal cancer (CRC) using flow cytometric analysis and light microscopy. The aim of the present study was to investigate the exact localization of the CEA-antigen on or in macrophages using transmission electron microscopy (EM) and immuno-electron microscopy (IEM). Besides, further insight in the macrophage role and its trafficking abilities in colorectal cancer patients is pursued, aiding to clarify more of the previous study findings.

\section{Methods}

Samples from vital tumour tissue and surrounding stromal tissue were collected from patients with colorectal carcinoma $(n=5)$. Samples were separated in two series. Series 1 was processed for regular EM to review the morphology and identify macrophages. Series 2 was used to visualize CEA in macrophages with IEM using gold-conjugated protein $A$ indirect immunolabelling. Furthermore, a second series of five different patients near-tumour lymph nodes were analysed by EM and IEM in the same way as described above.

\section{Results}

Using EM and IEM phagocytes, resembling macrophages, could be identified with intracellular CEA-positive gold particles connected to cell-debris in the cytoplasm. Secondly, anti-CEA-protein-A-gold-particles connecting to cancer-specific villi on the outside of cancer cells were detected. The initial lymph node assessment showed no CEA-positivity despite using multiple staining protocols.

\section{Conclusion}

In five patients the EM-aided morphology analysis provided a detailed structure of macrophage-resembling phagocytes, not only aiding to the recognition of macrophages using EM, but also providing insight in the possible locations of internalized CEA. Some intracellular vesicles e.g. lysosomes indeed showed cell debris. Using IEM, large multilobulated cored phagocytic cells (possibly macrophages) with CEA-positive cell-parts were visualised in some of the patients. These findings support our hypothesis of phagocytosis of CEA-containing (tumour) material by macrophages in tumour tissue and their surroundings. The uptake is not generally present in all macrophages, consistently with the findings in peripheral blood. The investigated lymph-node-group showed no positivity, possibly due to technical error(s) in sampling, storage, preparation or due to the selection itself. These findings were not in line with previous light microscopy findings, needing further future investigations. 


\section{Introduction}

In earlier studies shedded tumour material was shown to be internalised by macrophages in prostate cancer ${ }^{1-3}$ and colorectal cancer patients ${ }^{4}$. This observation could be qualified and quantified as PSA-positive and CEA-positive respectively by using multi-colour flow cytometry after applying standardised staining procedures. These internalised cytoplasmic materials could also be visualised with light microscopy after $\mathrm{PSA}^{2}$ and CEA-staining cytospins (Figure 3.1A (reprinted with permission) ${ }^{4}$ ), made of these flow cytometry samples. Macrophages appeared to internalise CEA-positive material from stromal or tumour tissue, subsequently, to return to the peripheral blood stream ${ }^{4,5}$ using a route that is still unclear. In a second study using light microscopy it was shown that in the tumour-stromal surroundings of CRC-patients not only CEA-positive macrophages are present, but also CK-positive, M30-positive and TKTL1positive macrophages ${ }^{6}$. In further experiments (Figure 3.1B) the targeted CD14+CD16+ monocytes could be flow cytometrically sorted (FACSAria, BD) and visualised and photographed using light microscopy (LM). Thus, evidence of irregularly shaped cells with large lobular nuclei, resembling tissue monocytes or tissue macrophages ${ }^{7}$ was confirmed.

To further investigate our hypothesis it is important to know whether the internalised material is still expressing CEA or whether it is the cell-surface itself, expressing CEA-epitopes after an internalisation process. Using light microscopy it remains uncertain what cell area or cell part is exactly positive for this staining. Electron microscopy may be able to provide answers to this question if immunolabelling by antibodies can be transferred from flow cytometry to the EM modality. This paper describes the study protocol investigating the visualisation of internalised CEA-positive material in macrophages using transmission electron microscopy (TEM) and (cryo) immuno electron microscopy (IEM), and the first results thereof. 


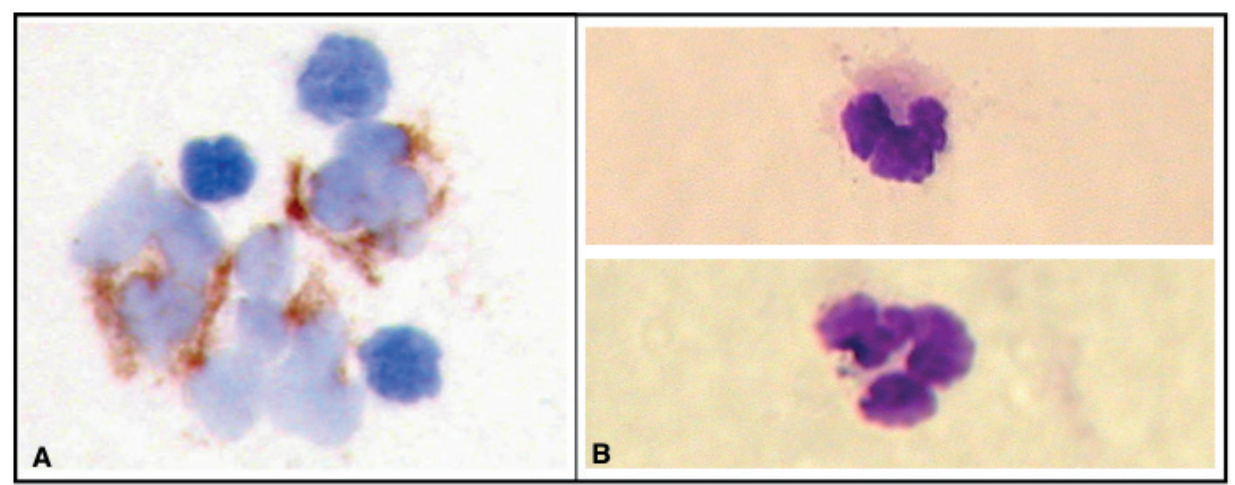

Figure 3.1 A) cytospin of CEA in macrophages (CEA-IM); an earlier published picture made of a cytospin of all cells from flow cytometry samples with brownish staining for CEA and H\&E staining for nuclear material. Large irregular cells with lobular nuclei clearly staining positive for CEA could be identified beside other cell-types. B) Flow cytometrically sorted (FACSAria, BD) CD14+CD16+ cell population showing only these large irregular cells with lobulated nuclei. The cells were sorted from samples of the standardised CEA-IM assay after regular workup for flow cytometry and Giemsa-stained after sorting and air-drying.

\section{Background}

\subsection{Macrophage and monocyte origin}

For a long time the kinetics of monocyte travel and differentiation to macrophages were thought to be based on a theory of Furth and Cohn, research from the 1960's. After spending some days in the bloodstream these monocytes would migrate into tissues replenishing the tissue-resident macrophage population ${ }^{8}$. Another theory of local macrophage renewal by proliferation was recently shown to be plausible. This was proven for particular tissuemacrophages such as the brain microglia or the skin Langerhans cells ${ }^{9,10}$. Macrophages are also proven not be depending on monocytes for replenishment in pancreatic, hepatic, pulmonary, splenic, peritoneal and bone marrow tissue ${ }^{11,12}$. On the other hand, also in the intestine influx of blood-borne monocytes and subsequent differentiation into tissue macrophages $\left(\mathrm{CX}_{3} \mathrm{CR} 1^{\mathrm{hi}}\right.$ type) appears to take place ${ }^{13}$. These cells (re)populate the intestine besides the already present self-replenishing resident macrophages (HLA+CD68+25F9+ type). Although earlier research stated that intestinal macrophages would have 
lost their CD14- and CD16-epitopes ${ }^{14,15}$, this statement refers to the resident intestinal tissue macrophages, not to the migrating blood-borne monocytes.

\subsection{Monocyte and macrophage internalisation process}

Macrophages are known for ingesting (invading) cells or cell-parts thereof and presenting parts of them on their cell-surface for signalling functions to other immune cells ${ }^{16}$ like such as T-cells ${ }^{17}$. They are also known for internalising cell debris after cell decay through endocytosis after which digestion takes place in lysosomes. If internalisation and subsequent digestion were not the mechanism involved but rather internalisation and cell-surface expression a signalling function would be more likely than a scavenger function. Re-localisation of nondifferentiated monocytes after finalizing an internalisation process to regional lymph nodes has recently been confirmed ${ }^{18}$.

\subsection{Applicability to this protocol}

Our theory of extravasation of blood-borne monocytes into stromal areas around tumours and performing internalisation and digestive tasks fit in the above literature description on origin and function. In our hypothesis and in recent literature macrophages ${ }^{5}$ and/or activated monocytes ${ }^{19}$ have the ability to return to the blood stream, also after internalising cell debris. Alternatively, they could be able to travel to adjacent lymph nodes ${ }^{18}$.

\section{Materials and methods}

\subsection{Tissue sampling}

After obtaining medical ethical approval and informed consent, two groups of five patients were included. This setup was chosen to first determine the ability to apply the immunochemistry with anti-CEA to the epitopes after (cryo)fixation before starting tissue sampling of lymph node material. From the first patient group tissue samples were taken from CRC tumour-specimens within minutes after resection. Each specimen was inspected by a pathologist for optimal tissue sample selection. Sampling was performed from vital tumour tissue as well as from surrounding stromal tissue. Subsequently the tissue samples were fixed using two fixation strategies: one for morphologic imaging purposes $\left(1 \mathrm{~mm}^{3}\right.$ tissue-pieces fixed in 2,5\% glutaraldehyde (GA) in 0,1M phosphate buffer (PB) until slide preparation, (see further details in section 2.2.1)), another for (cryo) 
immuno-EM purposes $\left(\left(1 \mathrm{~mm}^{3}\right.\right.$ tissue-samples fixed for a maximum of 60 minutes in GA $0,2 \%$ + paraformaldehyde (PFA) $2 \%$ in $0,1 \mathrm{M} \mathrm{PB} \mathrm{pH} \mathrm{7.4,}$ followed by transition to $1 \% \mathrm{PFA}$ in $0,1 \mathrm{M} \mathrm{PB} \mathrm{pH} 7.4$ until further processing (see for further details in section 3.2.3)).

After microscopic histopathological evaluation of CRC resection specimen, extra LM sections were processed to evaluate the best EM-sampling areas (see section 3.1.1). From the second group of five patients samples of lymph node were extracted within minutes after resection and stored as described above, again combined with routine pathological analysis providing extra LM sections to evaluate the best EM-sampling areas (see section 3.1.1).

\subsubsection{Light microscopy area selection}

Extra sections of regular pathological analyses were used to evaluate the best EM-sampling areas for both series. These sections were digitalised using a Mirax scanner (3D-Histech, Hungary). Areas of interest were marked by one of the researchers (Pannoramic Viewer, 3D-Histech, Hungary) and confirmed or adjusted by a pathologist. Areas of interest were defined as containing tumour tissue as well as surrounding stromal tissue for series one and specific lymphoid node areas for series two. The probability of finding macrophages containing internalised shedded tumour-cell remnants was considered the highest in these areas. The marked areas were used to aid search guidance for the electron microscopist.

\subsection{Electron microscopy}

\subsubsection{Slide preparation for morphology evaluation using TEM}

Tissue samples of both series of 5 patients were fixed in $2.5 \%$ glutaraldehyde in $0.1 \mathrm{M}$ phosphate buffer solution and embedded in Epon (LADD, US). The tissueblocks were cut into $80 \mathrm{~nm}$ sections for transmission electron microscopy (TEM) and photographed in the CM100 transmission electron microscope (Philips). The embedding process contained several steps. First, the samples were put in $0.1 \mathrm{M}$ phosphate buffer (PB) for 30 minutes, after which $1 \%$ osmium tetroxide was added for $60 \mathrm{~min}$. Second, after rinsing for $45 \mathrm{~min}$ in $0.1 \mathrm{M}$ PB for $45 \mathrm{~min}$ the tissue was dehydrated in a series of increasing ethanol concentration $(70 \%$, $90 \%, 100 \%, 100 \%)$. The tissue was then routinely processed for embedding in Epon resin. From these Epon blocks $1 \mu \mathrm{m}$ sections were cut and stained with toluidine-blue (Merck) to enable light microscopical navigation to the areas of interest prior to the EM search according to the earlier LM-defined areas of 
interest. Then the ultrathin sections of $80 \mathrm{~nm}$ were cut and stained with uranyl acetate and lead citrate before evaluation and picturing in the CM100 transmission electron microscope (Philips).

\subsubsection{Slide evaluation and cell selection using TEM}

An experienced electron microscopist evaluated the prepared sections using TEM and identified phagocytes (monocytes and macrophages) through predetermined cell aspects such as cell size, cell shape, nuclear lobulation and the presence of lysosomal organelles in the cytoplasm.

\subsubsection{Slide preparation for Immuno-EM (IEM) and staining protocols}

Tumour-, stromal- and lymphoid tissue samples were prepared according to the Tokuyasu-method $^{20,21}$. In short, the tissue samples were extracted from the storage solution (PFA in PB), rinsed with $0,15 \%$ Glycine in PBS 0,1 M, followed by immersion steps in gelatine $2 \%, 5 \%$ and $10 \%$ respectively for $15-30$ minutes, leaving it to harden in the refrigerator. Before cutting, 1\% PFA+0.1 M PB pH 7.4 was applied (for minimal $1 \mathrm{hr}$ ) and the tissue blocks were cut on ice in $1 \mathrm{~mm}^{3}$ blocks and transferred to $2.3 \mathrm{M}$ sucrose solution for overnight incubation (19.68 gram sucrose in $25 \mathrm{ml} 0,1 \mathrm{M} \mathrm{PB} \mathrm{pH} \mathrm{7.4)}$ for cryo-protection. Pieces were taken out of sucrose solution and pinned. The sucrose surplus was removed and subsequently stored in nitrogen ready for further staining after cutting.

In a first try-out, an anti-CEA/protein A-Gold labelling was applied. Using an ultramicrotome $80 \mathrm{~nm}$ sections were cut and placed on a 75 mesh copper grid. The sections were placed in a droplet of methyl-cellulose/2.3 M sucrose solution after which a cell staining protocol was started. The ultrathin sections were removed from the methyl-cellulose $/ 2.3 \mathrm{M}$ sucrose solution and placed in $2 \%$ gelatin in PBS in a petri dish for 10 minutes at $37^{\circ} \mathrm{C}$, followed by 2 times 2 minutes of $0,15 \%$ glycin in PBS, and subsequently by $0,1 \%$ Bovine Serum Albumin (BSA)/PBS for 2 minutes. Primary incubation was then performed in a solution containing an antibody directed against the CEA-antigen (IgG1, clone II-7, $270 \mathrm{mg} /$; Dako, Glostrup, Denmark) which was added for at least 20 minutes followed by 4 times 2 minutes rinsing with 0,1\% BSA/PBS. This was followed by secondary incubation with protein A-gold ${ }^{22}$ in 1\% BSA/PBS for 20 minutes. Rinsing with $0,1 \%$ BSA/PBS twice for two minutes was followed by rinsing twice for two minutes using only PBS. Rinsing using GA 1\% in PBS for 5 minutes was used for protein stabilisation after which six times rinsing for one minute with demineralized water (Milli-Q) was applied. The grid was moved to an iced bath containing 1.8\% methylcellulose/0.4\% Uranyl acetate (MC/UA) for 
5-10 minutes. After rinsing it in ice-cold 1.8\% MC/0.4\% UA, the grid was removed and the surplus of MC/UA was diminished to a film layer using filtration paper. The sections were finally air-dried.

In a second try-out the primary antibody directed against CEA was used in combination with a $25 \mathrm{~nm}$-gold conjugated goat-anti-mouse antibody against IgG1 as a secondary antibody (Aurion, Wageningen, The Netherlands). The same preparation protocol was used as in the first try-out.

In order to establish that the EM-determined phagocytes were indeed macrophages simultaneous labelling for CEA and a macrophage marker in the same cells was attempted. For that purpose the same markers were used as in previously published peripheral blood analyses ${ }^{4}$. Anti-CD14-APC (clone Mø P9, $25 \mu \mathrm{g} / \mathrm{ml}$; B\&D) and anti-CD16-FITC (clone NPK15, $125 \mu \mathrm{g} / \mathrm{ml}$; B\&D) were used. Staining performance of both anti-CD14- and anti-CD16-antibodies was tested on CRC-tissue and evaluated by LM first. Only the anti-CD16-FITC-antibody was proven to be possibly applicable in the $25 \mathrm{~nm}$-gold-conjugated goat-antimouse-antibody protocol. The anti-CD14-antibody was not used for EMpurposes due to LM-staining issues reported in de results-section 4.2.3.

\subsubsection{Slide evaluation and cell selection using IEM}

The same experienced electron microscopist evaluating the TEM-slides evaluated the IEM-slides searching for the same large irregular cells with lobular nuclei and lysosomal sub-cellular organelles. Single staining for CEA and CD16 was assessed by searching for $25 \mathrm{~nm}$ gold spheres suggesting presence of the CEA or CD16 epitope.

\section{Results}

\subsection{Light microscopy}

\subsubsection{Area selection}

Using regular light microscopy areas of possible macrophage infiltration were digitally tagged. This resulted in multiple slides per patient with several areas to investigate using TEM and IEM. In Figure 3.2 an example of an area of interest is shown within a light blue square. These selected area were all evaluated and confirmed by a pathologist. Parts of the selection were cut out from the tissue 
samples and prepared for electron microscopy evaluation (see section 3.2.1 and 3.2.3).

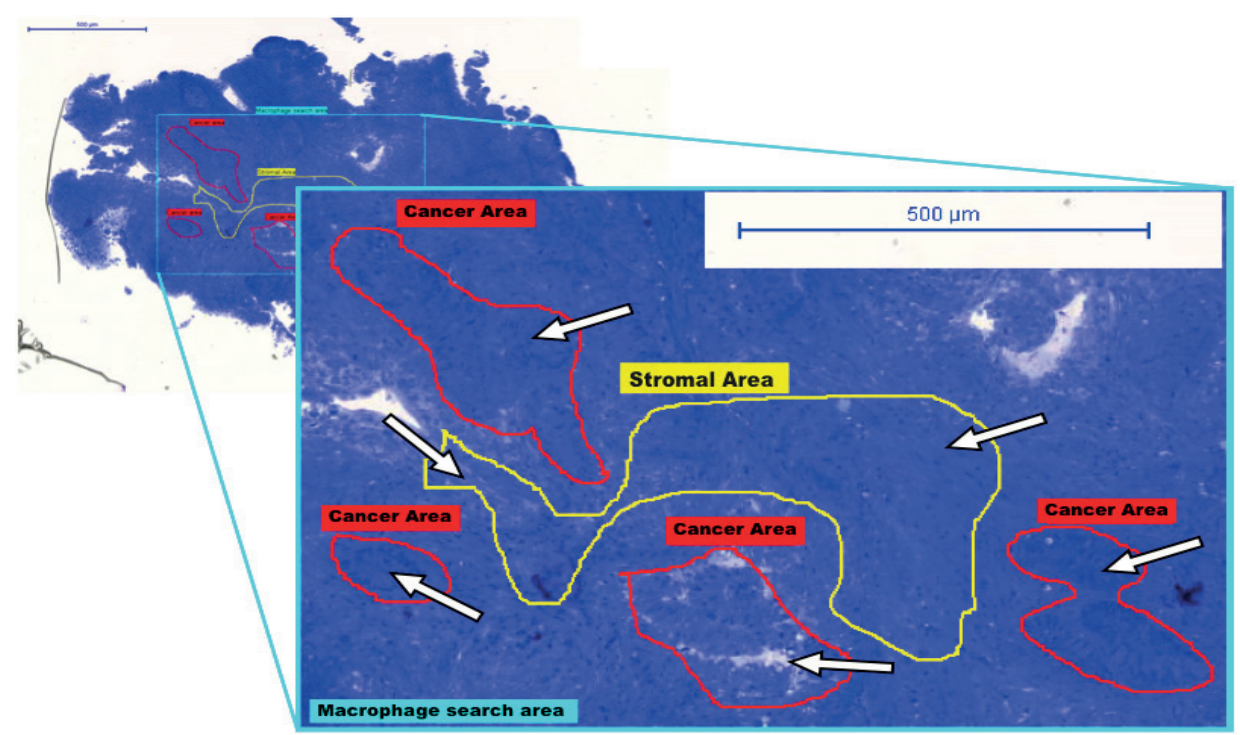

Figure 3.2 An example of a selected area where phagocytes most likely reside (light blue rectangle) and where electron microscopical evaluation could detect internalised tumour-related material (arrows). The red area marks vital CRC tissue, the yellow area marks a part of the stromal tissue area.

\subsection{Electron microscopy}

\subsubsection{Cell identification by TEM based on morphology}

Multiple phagocytes were identified in both tumoural and stromal areas (Figure 3.3) in the first group. The TEM-determined phagocytes were used for comparison in the evaluation of the (cryo)-immuno-EM sections. 
Chapter 3
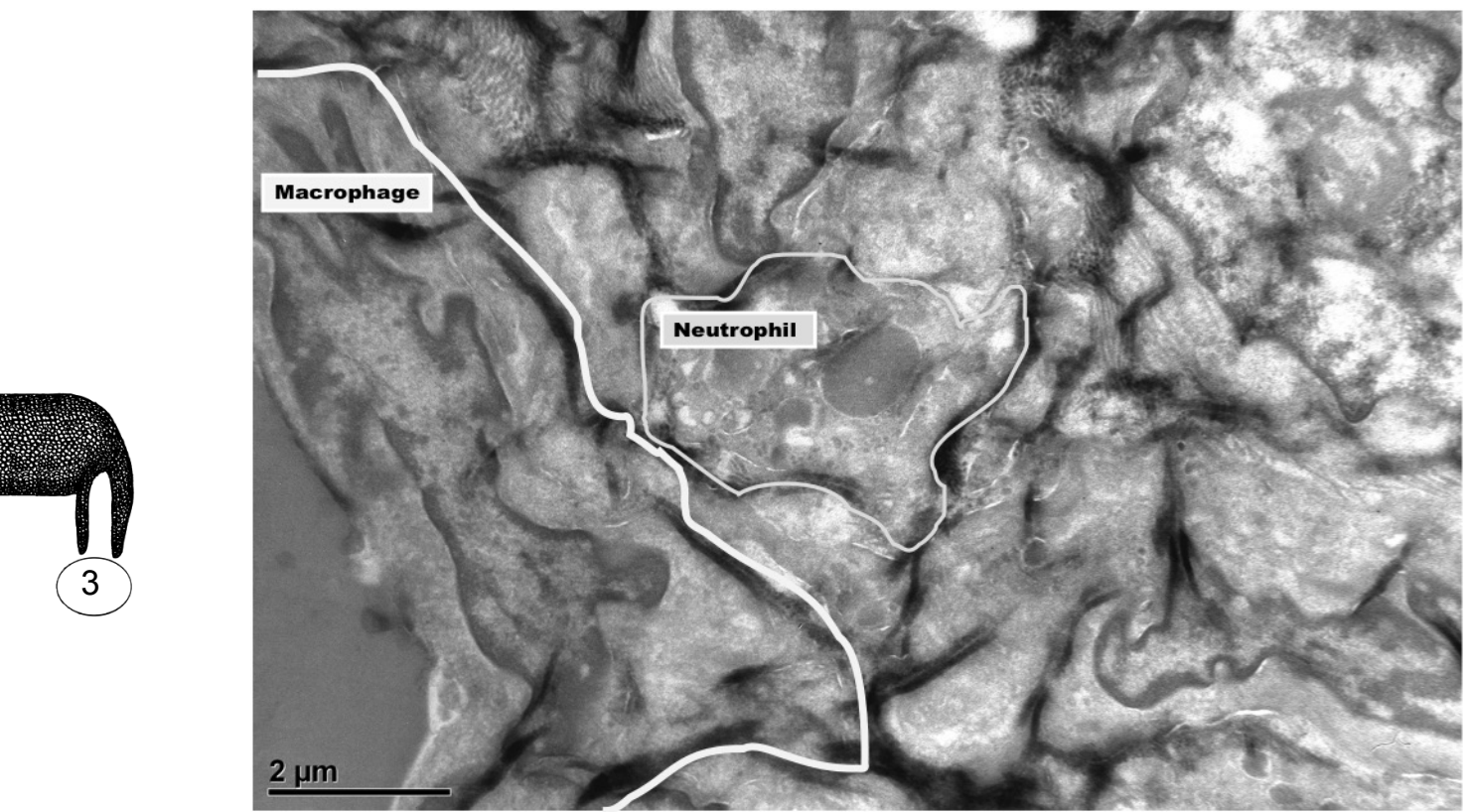

A

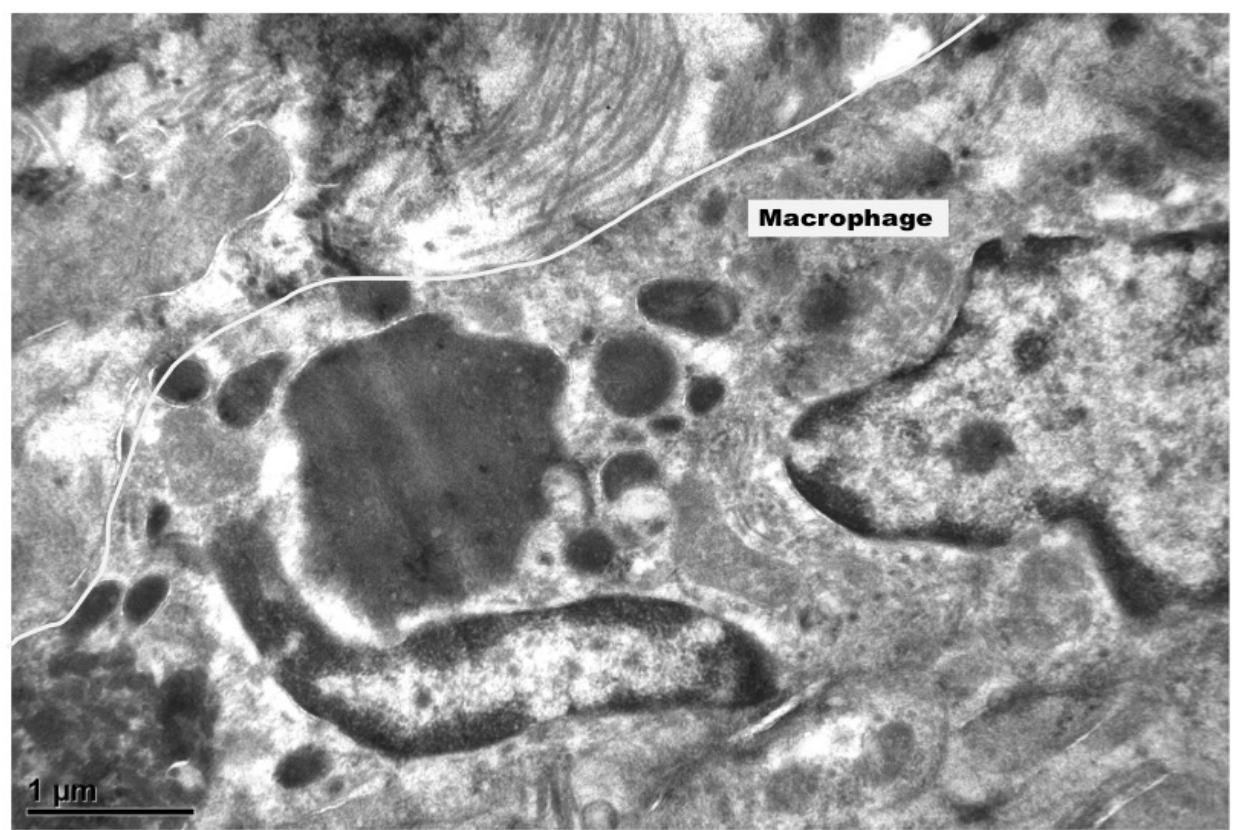

B

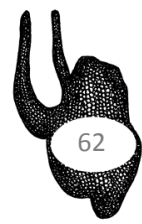


Figure 3.3 TEM-based selection areas of (A) tumour- and (B) stromal tissue respectively. In both areas phagocytes reside. They can be identified by size, shape, cytoplasmic vacuoles and a lobular nuclear configuration. In section $\mathbf{A}$ the total disruption caused by tumoural growth is obvious. In the lower left and center of the section two very irregularly shaped cells can be identified between tumour tissue. In the center the cell resembles a neutrophil while the one in the lower left corner seems to be a monocyte or a macrophage. In section B a stromal section is captured, showing connective tissue fibers in the upper part of the section and two parts of a nucleus derived from a macrophage in the right en lower center part. The macrophage almost extends over the complete section under the diagonal line, which can be drawn starting beside the connective tissue fibers top-center to the left edge (see marking).

In Figure 3.4 an example of a phagocyte is photographed.

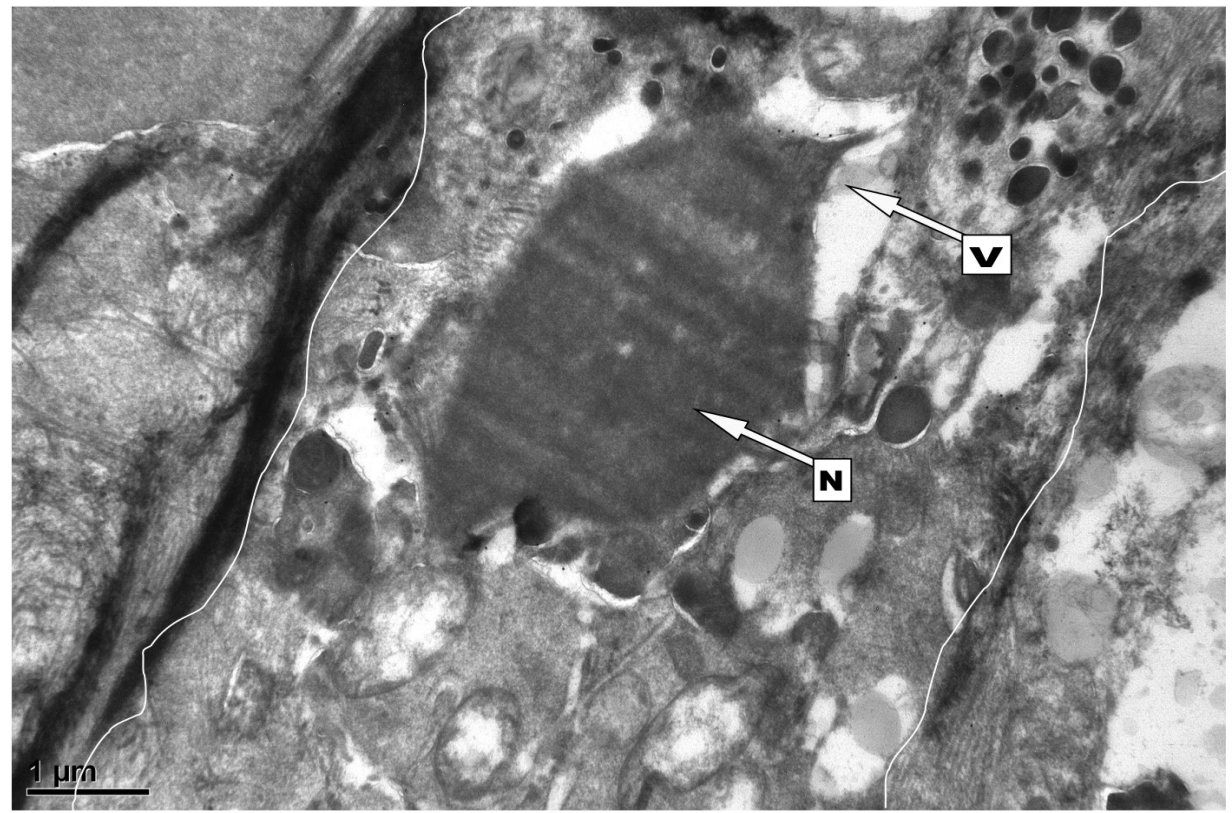

Figure 3.4 A phagocyte (white marking) containing an internalised nucleus $(\mathrm{N})$ inside a vesicle $(\mathrm{V})$ surrounded by cell organelles in the cytoplasm. The ingested nucleus seems to be degrading, characterized by indentations of nuclear surface.

\subsubsection{Phagocyte selection by IEM based on CEA-positivity}

Using the results of the TEM the concurring cell types were searched in the IEMslides stained with anti-CEA (according to the protein-A-protocol). Due to another fixation process the morphology of the cells in IEM was changed, resulting in more difficulty to find the proper cells than in the TEM procedures. 
Despite this, also in the IEM-slides phagocytes were found. Some but definitely not all showed a positive staining with anti-CEA. Next to the positive staining of phagocytes, many tumoural villous cell linings showed positivity as well (Figure 3.5).

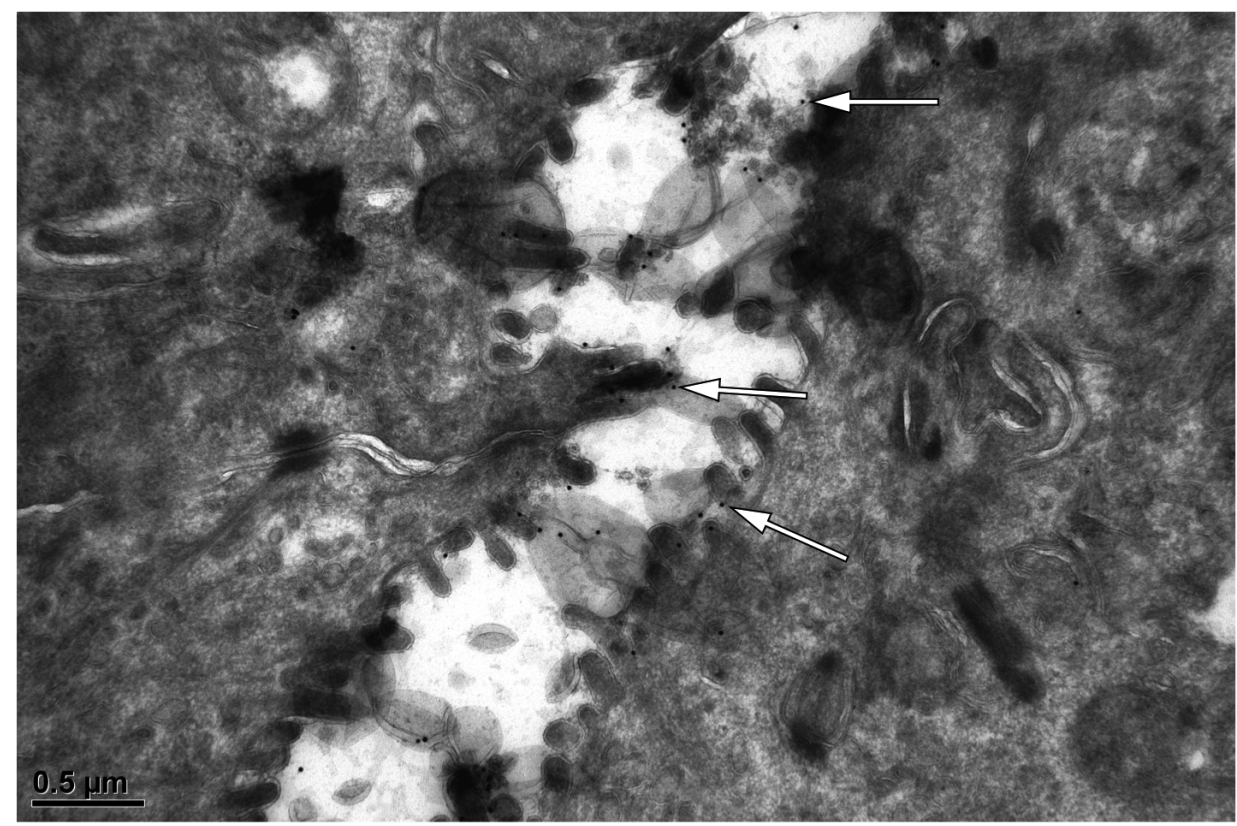

Figure 3.5 This section is selected from a colorectal cancer tumour site. Tumour tissue is seen on both sides of the villous cell lining. On top of the villous lining gold spheres confirm presence of CEA (white arrows). Also some concentrated gold spheres can be found deeper into the tumour tissue on the left side below the largest darker area. Further scattered over the total section some gold spheres can be identified. These gold spheres indicate the presence of CEA in the tumour tissue.

In several slides CEA-positive material was detected inside phagocyte-like cells. In Figure 3.6 an example is shown. 

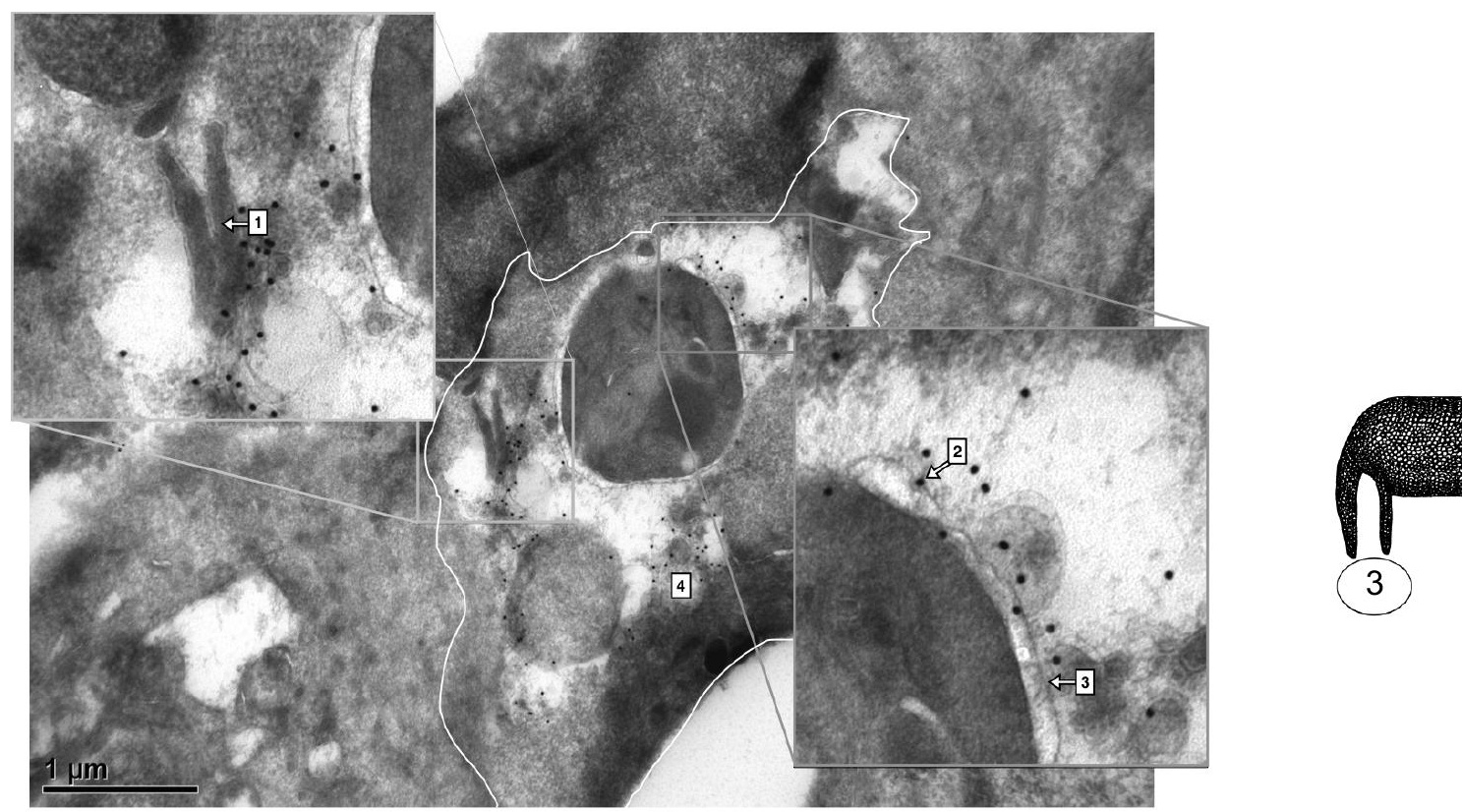

Figure 3.6 Example of a phagocyte (white irregular marking) characterised by appearance of an irregular shaped cell containing a multi-lobulated irregular nucleus (by two light grey irregular circles, possibly explained by dissection plane), showing multiple cell-partremnants like some villi-parts (1) and gold spheres collected on a part of a nucleus (2). The villi suggest being a part of gastro-intestinal lining, possibly tumour epithelium. The nuclear remnant appears to be surrounded by a vesicle wall (3), possibly a layer of phagocyte cell wall, which is internalised after egulfment of the remnant. Other localized collections of gold spheres can be objectified suggesting presence of CEA in internalized tumour material (4). Not being obviously encapsulated in a vacuole it could resemble part of Golgi apparatus transporting some tumour related material in a further digested state where the edges of the material are not as sharp anymore.

\subsubsection{Phagocyte selection by IEM based on double staining}

Immunochemical staining of CRC-tissue for CD14 or CD16 to identify (activated) monocytes respectively was attempted in sections evaluated by LM to predict feasibility of these immunochemical stainings in EM. The LM sections showed positive staining on macrophages with the antibody directed against CD16 (data not shown) However, staining with antibodies against CD14 did not deliver any positive results in LM. The antibody directed against CD14 appeared not to be suitable for fixed tissue samples and was therefore not attempted in EM. The staining with the anti-CD16-antibody was attempted solitary in EM at first. These sections did not show any positive results, not even after applying different 
concentrations of anti-CD16-antibody. Visualising CEA-positive tumour related material by double staining for CEA along with CD14 and/or CD16 (concurring with the flowcytometric blood-analysis of activated CD14+CD16+ monocytes) was not attempted due to these negative results.

\section{Discussion}

\subsection{Main findings}

This study shows the ability to detect phagocytes that have internalised tumour related cell remnants (e.g. tumour lining or nucleic material) in both stromal and tumour tissue using transmission and immuno-electron microscopic techniques. The cells presented can be recognised as tissue macrophages or tissue monocytes due to their specific morphology. The presence of CEA-positive internalised material proves the ability of the phagocytes to retain CEA-positive material at least for some time to be able to stain and visualise this material. The assumption that phagocytes (e.g. macrophages) digest all internalised material completely, prohibiting detection of internalised material, seems not to apply to this chosen setup within the set timeframe. The localisation of the tumour related material is inside the cytoplasm of the phagocytes, most likely in a lysosome, vacuole-like structure or Golgi apparatus, but definitely not on the cell surface of the phagocyte. An example of CEA-positivity on the cell surface is clearly seen on top of tumour-villi.

In addition to these key-findings this study identifies concurrent results with flow cytometrical analysis as the experiments show varying results between patients ${ }^{4}$. Only two out of five patients showed positive staining for CEA in several slides in the first series (using the protein-A-protocol), which is concurrent with findings in earlier immunohistochemical LM-studies ${ }^{6}$. In different areas different staining patterns occur, seemingly random, possibly based on occasional phagocyte presence.

Peripheral blood analyses show mostly reproducible results between and within patients and healthy controls. From these studies it is known that only a minor fraction of the extracted CD14+CD16+ blood monocytes are positive for internalised tumour material ${ }^{23}$. Considering these facts, tissue specimens from a larger cohort of CRC-patients could provide more representative outcomes preventing possible selection bias. In the next sections we describe the need for technical improvements. 


\subsection{CRC tissue extraction}

A challenge in sample preparation consisted of time-to-fixation after tissue specimen extraction. The time frame of transport of the tissue from the operating room (OR) to the pathology department took around 10 minutes where immediate tissue selection and extraction by a dedicated pathologist was available. This time frame is acceptable due to the toughness of the CEAepitope that will remain available after even a longer period of time.

\subsection{LM area selection}

Controlled area selection of probable macrophage-rich tissue using LM allowed a reduction of the search area, allowing quicker results and reducing possible erroneous cell identification. Transferring identification criteria of macrophages from LM to TEM and further to IEM facilitated identification of phagocytes in the changed morphology of the (cryo)-EM. The outcomes of each series were discussed and agreed on between the main researchers. However, transferring the preferred search area from one modality to the next remains a weak spot as one is not able to search exactly the same area because of possible tissue loss in cutting consecutive sections.

\subsection{Antibody challenges in IEM}

Epitopes in blood monocytes remain stable within 6-8 hours for staining using CD14, CD16, and CEA-antibodies ${ }^{23}$. In tissue, this will be similar when tougher fixation with GA and PFA would not be necessary for overall preservation. However, the evaluation of feasibility of using same CD14- and CD16-antibody as in flow cytometry was negative for the antibody against CD14 when staining LM sections of formalin fixed CRC-tissue. This is most likely due to protein cross-linking, which occurs during the fixation protocol. The fixation leads to disruption of the targeted epitope. The CD14-antibody used in the flow cytometric studies by our group is indeed designed for fresh or fresh frozen cell labelling. Because even tougher fixation protocols are applied in EM (with also aldehyde-derived fixatives), the application of this antibody becomes less feasible in EM. The use of the CD16-antibody instead for detection of phagocyte activation status remains an option, but can be debated. This antibody is directed against the Fcy-receptor IIIb, This transmembrane receptor is not only present on monocytes, but also on neutrophilic granulocytes, natural killer cells and mast cells. Whilst neutrophils are known for phagocytic capacity, the 
neutrophil cell morphology differs substantially from macrophages ${ }^{24}$ preventing recognition mistakes. The ability to identify activated phagocytes internalising CEA-positive cell material could have enabled to point out CEA-IM with more confidence. In LM the anti-CD16-antibody was properly visible (data not shown), but in TEM the anti-CD16-antibody did not provide any positive results, possibly due to the more aggressive tissue fixation protocol with glutaraldehyde. The proposed double staining using an anti-CD16-antibody conjugated to $15 \mathrm{~nm}$ gold spheres and an anti-CEA-antibody conjugated to $25 \mathrm{~nm}$ gold spheres was never attempted due to the EM-results of the CD16-staining in tissue in the second series. Activation of monocytes could therefore not be confirmed by using the antibody against CD16. Only morphology and CEA-positivity could be used for proper identification of the monocytes.

One could have opted for other markers like CD68 or CD11b to identify macrophages. Sadly these markers have not been applied in our flow cytometric assays before, therefore being second choice for now. Also the M1 or M2 macrophage-markers CD86 or CD163 respectively could provide answers in cell recognition in future experiments, but the experiences of these markers on the specificity to the targeted cells in flow cytometric assays should be gathered before application in EM.

Protein A-Gold labelling as detection method exhibits some known pitfalls ${ }^{25}$, e.g. nonspecific staining which in turn might lead to false-positive identification of CEA-IM. For that reason, the second strategy using a goat-anti-mouse antiIgG1-antibody conjugated with gold spheres should exclude this nonspecificity. A new series of sections of the proven CEA-positive tissue-samples from the first round (protein-A-series) combined with the second series (consisting of mere lymph-node-material) did not show any positivity in either section for CEA as well as CD16 using this new strategy. Firstly these outcomes prompted for critical evaluation of the new technique and secondly the outcomes of the first series using the protein-A protocol were questioned. Could every positive immunolabelling in the first series be due to coincidence or nonspecific staining? Was the second technique not suitable for the used tissue-samples or antibodies or were applied sampling logistics prohibiting proper results? Further improvement of the double immunostaining could provide these answers, after further optimising the sampling logistics and re-assessing staining protocols. 


\subsection{Future studies and conclusion}

Future studies applying other specific markers for the flow cytometrical detection of monocytes/macrophages (e.g. CD64, CD68, CD11b etc) as well as optimized EM fixation and staining strategies could further clarify the variable observations of CEA-positive material in macrophages and provide answers to questions remaining after the first part of this explorative study. 


\section{References}

1. Herwig R, Horninger W, Rehder P, Klocker H, Ramoner R, Thurnher M, Pinggera GM, Gozzi C, Konwalinka G, Bartsch G. Ability of PSA-positive circulating macrophages to detect prostate cancer. Prostate 2005;62:290-8.

2. Leers MPG, Nap M, Herwig R, Delaere K, Nauwelaers F. Circulating PSA-Containing Macrophages as a Possible Target for the Detection of Prostate Cancer. Am J Clin Pathol 2008;129:649-56.

3. Herwig R, Mitteregger D, Djavan B, Kramer G, Margreiter M, Leers MP, Glodny B, Haider DG, Hörl WH, Marberger M. Detecting prostate cancer by intracellular macrophage prostate-specific antigen (PSA): a more specific and sensitive marker than conventional serum total PSA. Eur $J$ Clin Invest 2008;38:430-7.

4. Japink D, Leers MPG, Sosef MN, Nap M. CEA in activated macrophages. New diagnostic possibilities for tumor markers in early colorectal cancer. Anticancer Res 2009;29:3245-51.

5. Joly $S$, Francke $M$, Ulbricht E, Beck $S$, Seeliger M, Hirrlinger P, Hirlinger J, Lang KS, Zinkernagel M, Odermatt B, Samardzija M, Reichenbach A, Grimm C, Remé CE. Cooperative phagocytes: resident microglia and bone marrow immigrants remove dead photoreceptors in retinal lesions. Am J Pathol 2009;174:2310-23.

6. Faber TJ, Japink D, Leers MP, Sosef MN, von Meyenfeldt MF, Nap M. Activated macrophages containing tumor marker in colon carcinoma: immunohistochemical proof of a concept. Tumour Biol 2011;33:435-41.

7. Ziegler-Heitbrock HW, Fingerle G, Ströbel M, Schraut W, Stelter F, Schutt C, Passlick B, Pforte A. The novel subset of CD14+/CD16+ blood monocytes exhibits features of tissue macrophages. Eur J Immunol 1993;23:2053-8.

8. van Furth R, Cohn ZA. 1968. The origin and kinetics of mononuclear phagocytes. J Exp Med 1968;128:415-35.

9. Ginhoux F, Merad M. Ontogeny and homeostasis of Langerhans cells. Immunol Cell Biol 2010; 88:387-92.

10. Ginhoux F, Greter M, Leboeuf M, Nandi S, See P, Gokhan S, Mehler MF, Conway SJ, Ng LG, Stanley ER, Samokhvalov IM, Merad M. Fate mapping analysis reveals that adult microglia derive from primitive macrophages. Science 2010;330:841-5.

11. Schulz C, Gomez Perdiguero E, Chorro L, Szabo-Rogers H, Cagnard N, Kierdorf K, Prinz M, Wu B, Jacobsen SE, Pollard JW, Frampton J, Liu KJ, Geissmann F. A lineage of myeloid cells independent of Myb and hematopoietic stem cells. Science 2012;336:86-90.

12. Hashimoto D, Chow A, Noizat $C$, Teo P, Beasley MB, Leboeuf M, Becker CD, See P, Price J, Lucas D, Greter M, Mortha A, Boyer SW, Forsberg EC, Tanaka M, van Rooijen N, GarcíaSastre A, Stanley ER, Ginhoux F, Frenette PS, Merad M. Tissue-resident macrophages selfmaintain locally throughout adult life with minimal contribution from circulating monocytes. Immunity 2013;38:792-804.

13. Zigmond E, Jung S. Intestinal macrophages: well educated exceptions from the rule. Trends in Immunology 2013;34:162-8.

14. Smythies LE, Shen R, Bimczok D, Novak L, Clements RH, Eckhoff DE, Bouchard P, George MD, Hu WK, Dandekar S, Smith PD. Inflammation anergy in human intestinal macrophages is due to Smad-induced IkappaBalpha expression and NF-kappaB inactivation. J Biol Chem 2010;285:19593-604.

15. Smythies LE, Sellers M, Clements RH, Mosteller-Barnum M, Meng G, Benjamin WH, Orenstein JM, Smith PD. Human intestinal macrophages display profound inflammatory anergy despite avid phagocytic and bacteriocidal activity. J Clin Invest 2005;115:66-75.

16. Jantsch J, Binger KJ, Müller DN, Titze J. Macrophages in homeostatic immune function. Front Physiol 2014;5:146. 
17. Leirião P, del Fresno $C$, Ardavín C. Monocytes as effector cells: Activated Ly-6Chigh mouse monocytes migrate to the lymph nodes through the lymph and cross-present antigens to CD8+T cells. Eur J Immunol 2012;42:2042-51.

18. Jakubzick C, Gautier EL, Gibbings SL, Sojka DK, Schlitzer A, Johnson TE, Ivanov S, Duan Q, Bala S, Condon T, van Rooijen N, Grainger JR, Belkaid Y, Ma'ayan A, Riches DW, Yokoyama WM, Ginhoux F, Henson PM, Randolph GJ. Minimal Differentiation of Classical Monocytes as They Survey Steady-State Tissues and Transport Antigen to Lymph Nodes. Immunity 2013;39:599-610.

19. Auffray C, Fogg D, Garfa M, Elain G, Join-Lambert O, Kayal S, Sarnacki S, Cumano A, Lauvau G, Geissmann F. Monitoring of blood vessels and tissues by a population of monocytes with patrolling behavior. Science 2007;317: 666-670.

20. Tokuyasu KT. A technique for ultracryotomy of cell suspensions and tissues. J Cell Biol 1973; 57:551-65.

21. Tokuyasu KT, Singer SJ. Improved procedures for immunoferritin labeling of ultrathin frozen sections. J Cell Biol 1976;71:894-906.

22. Slot JW, Geuze HJ. Sizing of protein A-colloidal gold probes for immunoelectron microscopy. $J$ Cell Biol 1981;90:533-6.

23. Japink D, Nap M, Sosef MN, Nelemans PJ, Coy JF, Beets G, von Meyenfeldt MF, Leers MP. Reproducibility studies for experimental epitope detection in macrophages (EDIM). $J$ Immunol Methods 2014;407:40-7.

24. Bain BJ. Blood cells a practical guide. Blackwell, Malden, Mass.; Oxford 2006.

25. Roth J, Taatjes DJ, Warhol MJ. Prevention of non-specific interactions of gold-labeled reagents on tissue sections. Histochemistry 1989;92:47-56. 



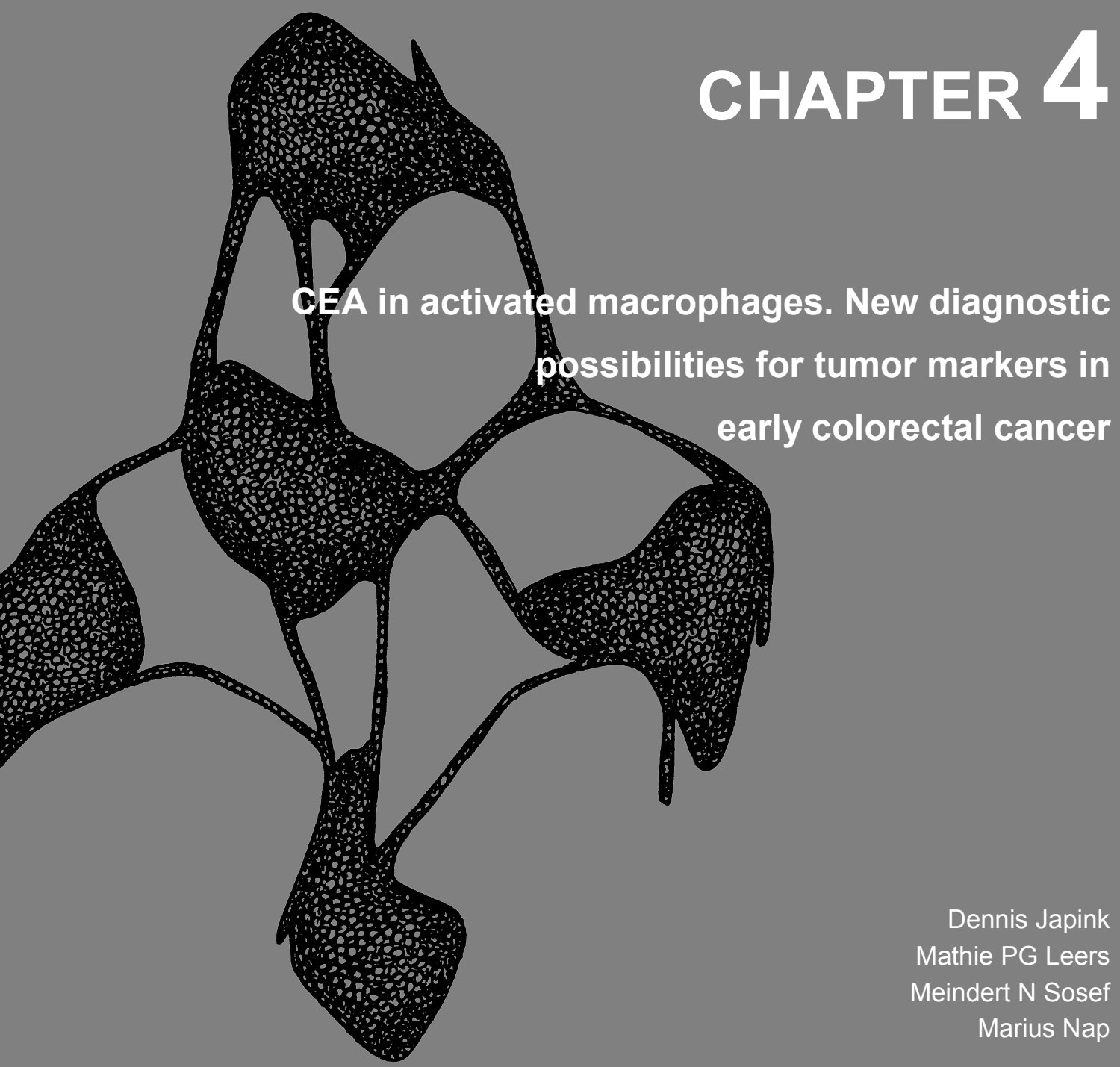

Anticancer Res 2009;29:3245-3251 


\section{Abstract}

\section{Background}

Serum tumor markers show low sensitivity, making them unsuitable for early detection of cancer. Activated macrophages (AM) from peripheral blood can accumulate tumor marker substances and facilitate early detection in prostate cancer. Here it was investigated whether carcinoembryonic antigen (CEA) containing macrophages (CEACM) can be used to detect colorectal cancer (CRC) at earlier stages than can serum CEA.

\section{Materials and methods}

Peripheral blood was collected from patients with CRC $(n=48)$, inflammatory colorectal disease $(n=5)$ and from healthy controls $(n=18)$. After separating and labeling AM with CD14-APC/CD16-FITC, AM were intracellularly labeled with anti-CEA antibody and flow cytometrically analyzed. Serum CEA and C-reactive protein (CRP) were measured.

\section{Results}

The fraction-size of CEACM discriminated between controls and CRC patients, irrespective of AJCC-stage (AJCC stage I-IV, $p=<0.0001$ ). Serum CEA values were significantly elevated in AJCC stage II, III and IV $(p=0.02,0.006$ and $<0.0001$, respectively). Combining CEACM with CRP-levels separated CRC from inflammatory colorectal disease.

\section{Conclusion}

CEACM combined with CRP appears to have diagnostic potential in early CRC. 


\section{Introduction}

Early detection of cancer in general is assumed to improve the possibilities for curative interventions and for this reason many efforts have been undertaken to achieve this goal. When Gold and Freedman presented carcinoembryonic antigen $(C E A)^{1}$, people had great expectations but in time learned that CEA serum assays cannot be used for the detection of colorectal cancer (CRC). Both sensitivity and specificity of this marker were insufficient to support this. This applies in general for all known tumor markers, with exception of human chorionic gonadotropin (HCG) and lactate dehydrogenase $\mathrm{LDH}^{2,3}$. Recently we improved the method published by Herwig et al. ${ }^{4}$ and demonstrated that activated macrophages, expressing both CD14 and CD16 on their membranes had ingested prostate-specific antigen (PSA)-containing material. The size of the fraction of PSA-containing macrophages correlated well with the presence of prostate cancer ${ }^{5}$. Activated macrophages can also be found as tissue macrophages in the interstitial space of many tissues and in particular in the stroma of neoplastic proliferations ${ }^{6}$. These cells have the capacity to migrate from peripheral blood and accumulate substances in tumor tissue and tumorsurrounding tissue $e^{7,8}$. If they can phagocytize PSA in prostate cancer, it could be hypothesized this mechanism also applies to other tumor markers in other types of tumors and may achieve increased diagnostic sensitivity as well. Although not specific, CEA is present in high amounts in CRC-tissue ${ }^{9}$. For this reason we have selected CEA as a candidate to test this hypothesis for colorectal cancer. We initiated a prospective study where we compared the presence of CEA in activated macrophages in different stages of cancer and inflammatory disease with serum CEA values and the level of CRP.

\section{Materials and methods}

Specimen collection: Medical ethical approval was obtained. Patients were included after histological confirmation of cancer on endoscopic biopsies was obtained and after informed consent. Consecutive inclusion was performed in the outpatient clinic or preoperatively on the ward. Classification in AJCC staging groups $^{9,10}$ was based on histopathological evaluation. Control persons were selected and included by matching the participating cancer patients for age and gender and if possible for social and environmental influences (e.g. spouses). Table 4.1 displays included patients and healthy controls. Two EDTA tubes, two serum tubes and one heparin tube of venous blood were collected. The heparin 
tube was used for determination of CRP. One serum tube provided a serum CEA level within three hours after the vena puncture, the other serum tube was centrifuged at $4^{\circ} \mathrm{C}$ and the supernatant was stored at minus $70^{\circ} \mathrm{C}$ for future analysis. One of the EDTA tubes was used for a complete blood count (CBC). The remaining EDTA-tube was kept at room temperature (RT) and processed for flow cytometry within 24 hours after vena puncture.

Table 4.1 Patient and control group demography.

$\begin{array}{lccr}\text { Group } & \text { Frequency } & \begin{array}{c}\text { Mean age } \\ \text { (years, (group range)) }\end{array} & \begin{array}{r}\text { No. Female } \\ \text { (\% in group) }\end{array} \\ \text { Controls } & 18 & 55.28(45-76) & 8(44.4 \%) \\ \text { Benign inflammatory disease } & 6 & 59.50(25-81) & 3(50.0 \%) \\ \text { Malignant } & 48 & 70.48(55-88) & 24(50.0 \%) \\ \text { Total } & 77 & 65.94(25-88) & 39(50.3 \%)\end{array}$

Mononuclear cell separation and immunocytochemical staining: One 3ml EDTA blood sample per patient was transferred into a CPT-tube (Becton Dickinson (B\&D), Franklin Lakes, NJ, USA). A 20-minute centrifugation of the blood sample at $1650 \mathrm{xg}$ isolated the mononuclear cells in a dense layer between the Ficoll-Hypaque density layer and the serum. This suspension of single cells was transferred into $100 \mu \mathrm{l}$ aliquots containing $\pm 10^{6} \mathrm{cells}$. To label the macrophages, directly conjugated monoclonal antibodies were added simultaneously. Allophycocyanin (APC)-conjugated monoclonal antibody against CD14 (clone M $\phi$ P9, $25 \mu \mathrm{g} / \mathrm{ml}$; B\&D) combined with a fluorescein isothiocyanate (FITC)conjugated monoclonal antibody against CD16 (clone NPK15, $125 \mu \mathrm{g} / \mathrm{ml}$; B\&D) were added and the cells were incubated in the dark at room temperature for 15 minutes. A Fix \& Perm strategy (GAS004, Caltag; InVitrogen, Carlsbad, CA, USA) was performed after a single $2 \mathrm{ml}$ phosphate-buffered saline (PBS) wash procedure. The fixation step consisted of the addition of $100 \mu \mathrm{l}$ fixation reagent $\mathrm{A}$ for 15 minutes at RT and two $2 \mathrm{ml}$ PBS wash steps afterwards. The permeabilization step was subsequently performed by adding $100 \mu \mathrm{l}$ permeabilization reagent $B$ in the presence of $20 \mu$ biotinylated anti-CEAantibody (clone II-7, $270 \mathrm{mg} /$; Dako Cytomation, Glostrup, Denmark), added for intracellular staining. Biotinylation and subsequent blocking was performed by using an Animal Research Kit (ARK, Dako Cytomation); calculating the needed biotin and blocking-reagent was based on the use of pure anti-CEA antibody Ig-concentration. A 15-minute incubation in the dark at RT preceded two 
washing steps with $2.0 \mathrm{ml}$ PBS. This was followed by incubation of the cell-pellet for 15 minutes at RT with phycoerythrin-conjugated streptavidin (streptavidinrPE, $0.5 \mathrm{mg} / \mathrm{ml}$; CAT No. 349023; B\&D).

\begin{tabular}{|lrrr|}
\hline Tube: CEA 20 & & & \\
Population & \#Events & \%Parent & \%Total \\
\hline All Events & 30,000 & & 100.0 \\
\hline Lymphocytes & 21,178 & 70.6 & 70.6 \\
Mononuclear cells & 7,216 & 24.1 & 24.1 \\
Monocytes & 6,532 & 90.5 & 21.8 \\
& 737 & 11.3 & 2.5 \\
Activated macrophages & 71 & 9.6 & 0.2 \\
\hline CEA containing macrophages & & &
\end{tabular}

Figure 4.1 Parental gating structure. Parental gating, starting at the level of mononuclear cells, resulted in a stepwise separation of sub populations of cells with accumulated complexity of phenotypical characteristics.
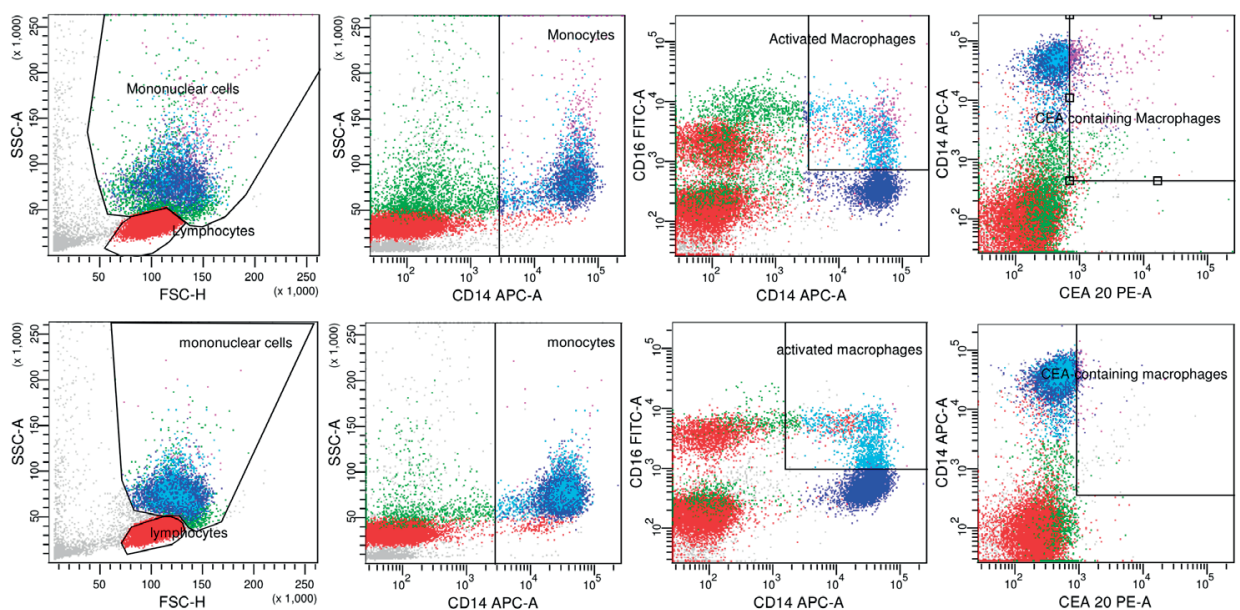

Figure 4.2 Gated sample of colorectal cancer patient (top row) and healthy control (bottom row). Parental gating is shown starting second plots from the left and in the two consecutive plots towards the right in both rows. More purple colored dots resembling CEACM are visible in the last, right most plot of the colorectal cancer patient compared to the healthy control.

After another two $2.0 \mathrm{ml}$ PBS wash-steps, the cell-pellet was resuspended in $0.5 \mathrm{ml}$ PBS and ready for measurement. A negative control (NC) was prepared by incubating cells with non-relevant mouse immunoglobulins which were 
directly labeled with -FITC (10.0 $\mu \mathrm{l} \operatorname{lgG}_{1}, \mathrm{~K}-\mathrm{FITC}$, clone G18-145; B\&D (Pharmingen)) and -APC (2.5 $\mu \mathrm{l} \operatorname{lgG}_{1}, \mathrm{~K}-\mathrm{APC}$, clone G18-145; B\&D (Pharmingen)) to reveal background staining by the directly conjugated primary antibodies. Streptavidin-rPE was included in the negative control for detection of possible endogenous biotin binding.

Flowcytometrical analysis: The prepared samples were analyzed with a FACScanto flow cytometer (B\&D). Excitation of the cells was performed by a $488 \mathrm{~nm}$, air-cooled solid-state argon laser followed by a $17 \mathrm{~mW} 633 \mathrm{~nm}$ helium/neon laser. A 530-nm band pass filter enabled FITC-fluorescence detection, a $575 \mathrm{~nm} \pm 13$ (mean \pm SD) band pass filter provided PEfluorescence detection and APC-fluorescence was assessed by using a $660 \mathrm{~nm}$ \pm 10 (mean \pm SD) band pass filter. Seven-color setup beads (BD FACS; B\&D) were used on weekly basis for optimal device performance. Automatic compensation settings were obtained by using the FACScanto software (BD Biosciences). At least 30000 events were counted for each sample. APC, FITC and $P E$ signal-data were recorded with logarithmical amplification. BD FACSDiVa software (B\&D) was used for analysis of the acquired data; the flow cytometrist was blinded for any clinical information.

Gating procedure: Mononuclear cells were selected by drawing a gate in the forward scatter (FSC) / side scatter (SSC) plot to separate them from lymphocytes. Parental gating, starting at the level of mononuclear cells, resulted in a stepwise separation of sub populations of cells with accumulated complexity of phenotypical characteristics (Figure 4.1). Within the population of mononuclear cells, CD14-positivity identified the monocytes. In the following step, CD14/CD16-positive activated macrophages were gated within the monocyte fraction. Finally, step 3 resulted in identification of the fraction of CEAcontaining cells within the activated macrophage fraction (see Figure 4.2).

Biochemical assays: Serum CEA levels were determined on an Advia Centaur Immunochemistry Analyzer (Bayer Healthcare, Diagnostics Division, Tarrytown, NY, USA). The CEA-assay on the Bayer Advia Centaur was a 2-sided dual monoclonal sandwich immunochemiluminescent assay (reference values of $<5.0 \mu \mathrm{g} / \mathrm{l}$ (non-smoking population) and $<10.0 \mu \mathrm{g} / \mathrm{l}$ (smoking population) were considered normal). Serum CRP levels were tested by particle-enhanced immunoturbidimetric assay (CRPLX, Tina-quant, Roche Diagnostics $\mathrm{GmbH}$, 
Mannheim, Germany) (reference value of $<10.0 \mathrm{mg} / \mathrm{l}$ was considered normal) on a E170 Modular Analytics System (Roche Diagnostics, Hoffmann-LaRoche Inc, Basel, Switzerland).

Cytospin preparations and immunocytochemistry: After preparation of the cytospins, immunocytochemistry was performed for the detection of the CEAantigen, using the same monoclonal antibody as described above. For this purpose, an indirect streptavidin-biotin method was performed.

Statistical analyses: SPSS Statistical software, version 16 for Macintosh (SPSS Inc., Chicago, Illinois, USA) was used for the statistical analysis. The Mann Whitney $U$ test was used for analysis of differences. A p-value of less than 0.05 was considered statistically significant. Graphpad Prism, version 4.0 for Macintosh (Graphpad Software Inc., San Diego, California, USA) was used for creating graphs.

\section{Results}

The size of the fraction of activated macrophages did not differ between the investigated patient groups with CRC (Figure 4.3). However, we observed that AJCC stage III (mean \pm SD: $32.68 \% \pm 11.46$ ) shows significantly higher fractions of activated macrophages compared to healthy controls $(20.57 \% \pm 8.12, p=0.008)$, stage I and stage II colorectal cancer $(21.20 \% \pm 8.46, \quad p=0.028$ and $21.10 \% \pm 11.66, p=0.005$ ). In all patient groups with CRC (AJCC-stage: mean \pm SD) CEACM was significantly higher (stage I: $15.93 \% \pm 10.61$, stage II: $17.63 \% \pm 12.76$, stage III: $15.91 \% \pm 7.73$, stage IV: $16,54 \% \pm 8,03$, all $p<0.0001$ ) in comparison to healthy controls, $(2,86 \% \pm 1,19)$ (see Figure 4.3). The differences calculated comparing malignant disease with benign inflammatory disease were not significant; the difference between healthy controls and benign inflammatory disease $(15.72 \% \pm 22.85)$ was significant $(p=0.005)$. The pre-operative CRP levels of patients with benign inflammatory disease $(165.67 \mathrm{mg} / \mathrm{l} \pm 48.38)$ did significantly differ from patients with colorectal cancer (stage I: $3.80 \mathrm{mg} / \mathrm{l} \pm 3.63$, stage II: $14.75 \mathrm{mg} / \mathrm{l} \pm 17.85$ and stage III: $11.75 \mathrm{mg} / \mathrm{l} \pm 15$. 90 ; p-values of 0.024 , 0.034 and 0.034 respectively). Combining the measurements of activated macrophages, CEA-containing activated macrophages and pre-operative CRPlevels provides a patient group-specific marker profile also separating benign inflammatory disease from cancer (Figure 4.3). 


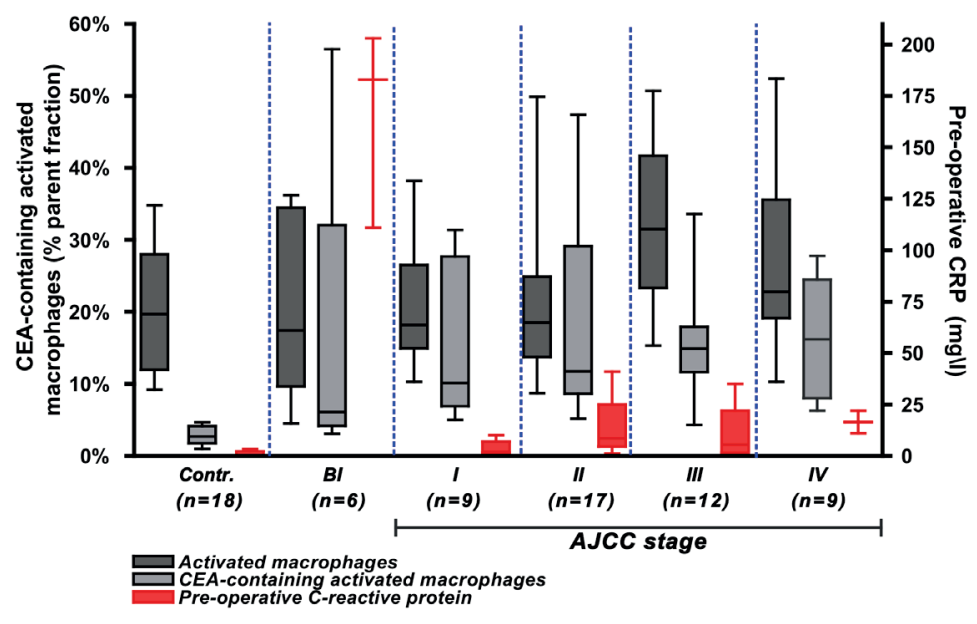

Figure 4.3 Patient group profiles showing fractions of activated macrophages, fractions of CEAcontaining activated macrophages and pre-operative CRP levels. Clearly the distinction between the healthy group and the benign group as well as all malignant groups can be made by measuring CEACM. CRP adds the ability to significantly differentiate between the benign inflammatory patient group and AJCC groups I-III.

Serum CEA analysis (Figure 4.4): The standard serum CEA assay showed significantly elevated values in AJCC stage II to IV CRC (stage II: $6.36 \mu \mathrm{g} / \mathrm{I}$ \pm 13.49 , stage III: $6.51 \mu \mathrm{g} / \mathrm{l} \pm 8.77$ and stage IV: $95.18 \mu \mathrm{g} / \mathrm{l} \pm 176.31$; $\mathrm{p}$-values of $0.02,0.006$ and $<0.0001$ respectively) in comparison to healthy controls $(1.45 \mu \mathrm{g} / \mathrm{l} \pm 0.70)$. In AJCC stage I $(2.10 \mu \mathrm{g} / \mathrm{l} \pm 2.05)$, the serum CEA value did not significantly differ from that of healthy controls. In most of the investigated patients (including CRC patients), the serum CEA assay showed outcomes lower than reference values, together with high variance within the CRC groups.

Photographic proof of CEA-containing macrophages: Immunocytochemical staining procedure of cytospins prepared from the monuclear cell fraction after CPT-tube centrifugation showed presence of vesicles staining positive for CEA in the peripheral zones of the cytoplasm of macrophages with large irregular nuclei (Figure 4.5). 


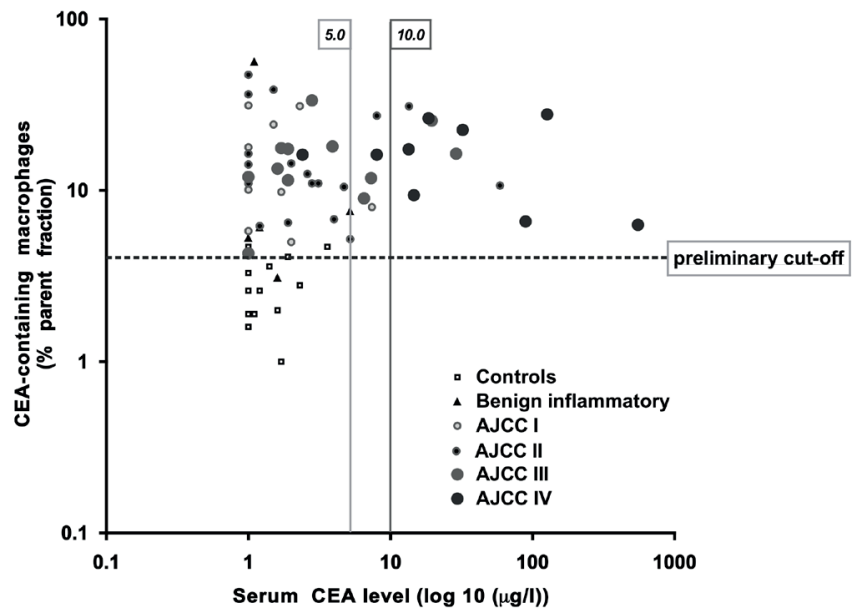

Figure 4.4 Comparison of the fraction of CEA-containing activated macrophages with serum CEA levels in AJCC staging groups. Cut-off values of 5.0 and $10.0 \mu \mathrm{g} / \mathrm{l}$ (healthy and smoking respectively) of the serum CEA assay have been displayed by vertical continuous lines. The graph shows almost only higher staging groups being detected by the regular serum CEA-assay, but not in most cases. The flow cytometrical assay shows much better distinction between early cancer groups and the healthy controls. The horizontal dashed line could be used as a cut-off value for distinction between healthy controls $(\leq 4.25-4.85 \%)$ and malignant disease $(>4.25-4.85 \%)$ depending on diagnostic demand. Sensitivity and specificity in this cut-off value range varied between $100.0-97.9 \%$ and $83.3-100.0 \%$ respectively.

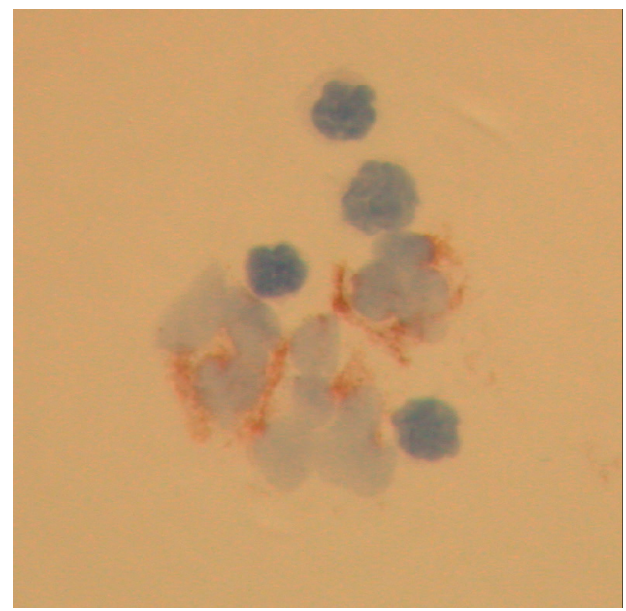

Figure 4.5 Cytocentrifuged sample stained for CEA prepared from an analysis sample used for flow cytometry. Visible are the bluish staining for the cell nucleus and the brownish antiCEA staining inside intracellular vesicles containing CEA. 


\section{Discussion}

Serum tumor markers were initially thought to be the ideal instrument for early detection of malignant tumors. However, low serum concentrations as a result of dilution effects in the total blood volume in combination with a majority of smaller primary tumors soon showed that only high-stage tumors or recurrent disease remained candidates for the use of these biomarkers ${ }^{11,12}$. Various committees tried to postulate guidelines for the use of tumor markers based on metaanalyses of international research in randomized controlled setting. The European Group on Tumor Markers (EGTM) ${ }^{11}$, the National Academy of Clinical Biochemistry (NACB) ${ }^{13}$, the European Society for Medical Oncology (ESMO) ${ }^{14}$ and comprehensive cancer centers provide guidelines and quality-assessments for the analysis process using serum tumor markers. In the current situation, these guidelines leave the clinician with a tool hardly applicable for use in major patient groups, leaving room for improvement. At the moment, the only application of serum CEA in CRC treatment guidelines is the use of this value in the follow-up of CRC. Not as a solitary marker, but as a small part of an intensive periodical follow-up strategy also containing modern imaging techniques, endoscopy and physical examination. It has been shown from metaanalysis that intensive follow-up strategy gains $7 \%$ in mortality reduction ${ }^{15-17}$.

The results of both our present study and earlier publications ${ }^{5}$ illustrate how intrinsic functions of a part of an organism such as tissue macrophages may be used to establish a test that provides insight in the state of health of the entire organism.

One of the hypotheses postulated (also by Gordon et al) is migration of macrophages to draining lymph nodes and no further. Apart from apoptosis, necrosis, autolysis and ingestion by other macrophages (also outside lymph nodes), they may release material for cross presentation by dendritic cells. In view of the similarity of activated macrophages in the peripheral blood to tissue macrophages $^{18}$, we postulate that the success of our method used here is based on a special condition. Activated macrophages are attracted to the tumor stroma and find themselves in close proximity to the damaged tissue. The accumulation of cell constituents inside these activated macrophages leads to concentration. Once saturated, the activated macrophages reach the peripheral blood and are sampled via venipuncture. A second concentration step is built into our assay, namely when the buffy coat is selected. The labeling of the activated 
macrophages with CD14/CD16 can be regarded as an additional selective step. The last step, where the relevant tissue component is labeled and detected, thus receives a huge boost in sensitivity, which might explain the surprisingly good separation of controls from even AJCC stage I tumors. When comparing serum CEA with the fraction-size of CEACM in all patient groups (Figure 4.4) again the potential diagnostic power of this new assay even in AJCC stage I is shown. In most cancer cases the new assay produced an elevated signal, whereas the serum CEA assay lacked this ability. At the time of writing reference values have not been established. However, our present data seem to provide a preliminary cut-off value of $4.25 \%$ (Figure 4.3 ) to distinguish healthy controls $(\leq 4.25 \%$ ) from malignant cases (>4.25\%). Using this cut-off, $100.0 \%$ sensitivity and $83.3 \%$ specificity was reached, already in AJCC stage I. Increasing the cut-off value to $4.85 \%$, the sensitivity decreased to $97.9 \%$, but the specificity increased to $100 \%$. The cut-off value should be chosen according to the diagnostic demand. These characteristics are better than the calculated sensitivity and specificity of currently used diagnostic possibilities ${ }^{16}$. These proposed preliminary cut-off values are of course dependent on the chosen gating strategy and may become different in future.

It appeared in our study that non-malignant disorders of the colorectal mucosa also resulted in increased fractions of CEACM. The inflammatory mechanism is not confined to tumorous development alone, but applies to all inflammatory processes in the body. Wherever tissue damage occurs, irrespective of the origin or the cause, the body reacts by starting this inflammatory reaction. The presence of CD16-positive monocytes, becoming CEA-positive, after celldamage in the colon-model indicates this inflammatory process has occurred.

During an inflammatory process (or exacerbation thereof) in the colon the normal lining of the colon will be shedded and the CEA, commonly present in healthy and inflamed colon, will shed as well. The macrophages in the colon-wall will be able to phagocytize the shedded gut-wall as well as the shedded CEA. These macrophages are hypothesized to return to the blood pool. For differentiation between benign inflammatory and malignant colon disease the combination with an inflammatory marker, CRP appeared to be a good option. High CRP levels are associated with active inflammation and/or tumor necrosis in higher disease stages, whereas stage AJCC I and II in general show no signs of inflammation (low CRP values). The percentage of activated macrophages in AJCC stage III is elevated in comparison to the lower disease stages and stage 
IV disease. A possible explanation for this may be that lymph node involvement in stage III disease stimulates the immune system, whereas in stage IV disease the immune system may be suppressed or exhausted.

Differences in macrophage involvement may also be due to the macrophageactivation state and macrophage reactions on entering this state. Upon an encounter triggering the macrophage, two possible reactions have been postulated in literature, the M1 and M2 reaction ${ }^{6,19}$. Phagocytosis for instance is performed by the M1-state macrophage, but angiogenesis is stimulated by M2state macrophages ${ }^{20}$. Phagocytosis of tumor remnants or even living tumor cells is of course desirable but angiogenesis to support tumor proliferation is obviously not. Furthermore, the substances a macrophage encounters differ depending on macrophage and location. The differences described above may explain why not all macrophages extracted from peripheral blood contain the same substances and also not the same amount thereof.

It can be concluded from data measuring $M 30$, an apoptosis marker ${ }^{21}$, in activated macrophages in CRC patients (manuscript in preparation) that this technique of detection of ingested substances in activated macrophages is applicable to whatever is technically detectable inside these tissue macrophages. This enhances the diagnostic applicability by a natural, preanalytical concentration step. A multi-marker strategy for solid tumors could be combined with this flow cytometrical approach, similar to the flow cytometrical analysis of patients with suspicion for bone marrow-associated malignancies.

Determining the patient profile (Figure 4.3) considering inflammatory serum parameter levels and perhaps multiple flow cytometrically obtained tumormarker-containing macrophage fractions leads to a more broad collection of cross-related information. This information may be suitable for predicting the origin of disease and enabling earlier detection of primary disease, at least providing incentive for additional invasive diagnostic procedures. In the context of activated macrophages, there may be a new diagnostic role for CEA in the early detection of colorectal malignancies. 


\section{References}

1. Gold P, Freedman SO. Demonstration of Tumor-Specific Antigens in Human Colonic Carcinomata by Immunological Tolerance and Absorption Techniques. J Exp Med 1965;121: 439-62.

2. Stieber $\mathrm{P}$, Heinemann V, Schalhorn A. Tumor markers--how they should be applied. MMW Fortschr Med 2005;147:35,37-39.

3. Reiter W, Stieber P, Reuter C, Nagel D, Lau-Werner U, Pahl H, Fateh-Moghadam A. Preoperative serum levels of CEA and CA 19-9 and their prognostic significance in colorectal carcinoma. Anticancer Res 1997;17:2935-8.

4. Herwig R PA, Horninger W, Rehder P, Klocker H, Ramoner R, Pinggera GM, Gozzi C, Konwalinka G, Bartsch G. Measurement of intracellular versus extracellular prostate-specific antigen levels in peripheral macrophages: a new approach to noninvasive diagnosis of prostate cancer. Clin Prostate Cancer 2004;3:184-8.

5. Leers MP, Nap M, Herwig R, Delaere K, Nauwelaers F. Circulating PSA-containing macrophages as a possible target for the detection of prostate cancer: a three-color/fiveparameter flow cytometric study on peripheral blood samples. Am J Clin Pathol 2008;129: 649-56.

6. Sica A, Allavena $P$, Mantovani A. Cancer related inflammation: the macrophage connection. Cancer Lett 2008;267:204-15.

7. Nakayama Y, Nagashima N, Minagawa N, Inoue Y, Katsuki T, Onitsuka K, Sako T, Hirata K, Nagata N, Itoh H. Relationships between tumor-associated macrophages and clinicopathological factors in patients with colorectal cancer. Anticancer Res 2002;22:4291-6.

8. Yuan A, Chen JJ, Yang PC. Pathophysiology of tumor-associated macrophages. Adv Clin Chem 2008;45:199-223.

9. Nap M, Wilhelm WW. Immunohistology of carcinoembryonic antigen (CEA). Specificity of reagents, choice of control tissues and practical application in surgical pathology. Int $J$ Biol Markers 1992; 7:148-53.

10. Compton C, Fenoglio-Preiser CM, Pettigrew N, Fielding LP. American Joint Committee on Cancer Prognostic Factors Consensus Conference: Colorectal Working Group. Cancer 2000;88:1739-57.

11. Duffy MJ, van Dalen A, Haglund C, Hansson L, Holinski-Feder E, Klapdor R, Lamerz R, Peltomaki P, Sturgeon C, Topolcan O. Tumour markers in colorectal cancer: European Group on Tumour Markers (EGTM) guidelines for clinical use. Eur J Cancer 2007;43:1348-60.

12. Duffy MJ, van Dalen A, Haglund C, Hansson L, Klapdor R, Lamerz R, Nilsson O, Sturgeon C, Topolcan O. Clinical utility of biochemical markers in colorectal cancer: European Group on Tumour Markers (EGTM) guidelines. Eur J Cancer 2003;39:718-27.

13. Sturgeon CM, Duffy MJ, Stenman UH, Lilja H, Brunner N, Chan DW, Babaian R, Bast RC, Jr., Dowell B, Esteva FJ, Haglund C, Harbeck N, Hayes DF, Holten-Andersen M, Klee GG, Lamerz R, Looijenga LH, Molina R, Nielsen HJ, Rittenhouse H, Semjonow A, Shih le M, Sibley P, Soletormos G, Stephan C, Sokoll L, Hoffman BR, Diamandis EP. National Academy of Clinical Biochemistry laboratory medicine practice guidelines for use of tumor markers in testicular, prostate, colorectal, breast, and ovarian cancers. Clin Chem 2008;54:e11-79.

14. Van Cutsem EJ, Oliveira J. Colon cancer: ESMO clinical recommendations for diagnosis, adjuvant treatment and follow-up. Ann Oncol 2008;19 Suppl 2: ii29-30.

15. Figueredo A, Coombes ME, Mukherjee S. Adjuvant therapy for completely resected stage II colon cancer. Cochrane Database Syst Rev 2008:CD005390.

16. Kievit J. Follow-up of patients with colorectal cancer: numbers needed to test and treat. Eur $J$ Cancer 2002;38:986-99. 
17. Rodriguez-Moranta F, Salo J, Arcusa A, Boadas J, Pinol V, Bessa X, Batiste-Alentorn E, Lacy AM, Delgado S, Maurel J, Pique JM, Castells A. Postoperative surveillance in patients with colorectal cancer who have undergone curative resection: a prospective, multicenter, randomized, controlled trial. J Clin Oncol 2006;24:386-93.

18. Ziegler-Heitbrock HW. Heterogeneity of human blood monocytes: the CD14+ CD16+ subpopulation. Immunol Today 1996;17:424-8.

19. Van Ginderachter JA, Movahedi K, Hassanzadeh Ghassabeh G, Meerschaut S, Beschin A, Raes G, De Baetselier P. Classical and alternative activation of mononuclear phagocytes: picking the best of both worlds for tumor promotion. Immunobiology 2006;211:487-501.

20. Geissmann F, Jung S, Littman DR. Blood monocytes consist of two principal subsets with distinct migratory properties. Immunity 2003;19:71-82.

21. Leers MP, Kolgen W, Bjorklund V, Bergman T, Tribbick G, Persson B, Bjorklund P, Ramaekers FC, Bjorklund B, Nap M, Jornvall H, Schutte B. Immunocytochemical detection and mapping of a cytokeratin 18 neo-epitope exposed during early apoptosis. J Pathol 1999;187:567-72. 


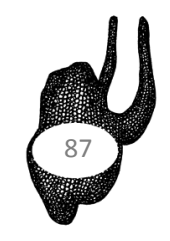





\section{Abstract}

\section{Introduction}

We have recently described epitope detection in macrophages (EDIM) by flow cytometry. This is a promising tool for the diagnosis and follow-up of malignancies. However, biological and technical validation is warranted before clinical applicability can be explored.

\section{Methods}

The pre-analytic and analytic phases were investigated. Five different aspects were assessed: blood sample stability, intra-individual variability in healthy persons, intra-assay variation, inter-assay variation and assay transferability. The post-analytic phase was already partly standardized and described in an earlier study.

\section{Results}

The outcomes in the pre-analytic phase showed that samples are stable for 24 hours after venipuncture. Biological variation over time was similar to that of serum tumour marker assays; each patient has a baseline value. Intra-assay variation showed good reproducibility, while inter-assay variation showed reproducibility similar to that of to established serum tumour marker assays. Furthermore, the assay showed excellent transferability between analyzers.

\section{Conclusion}

Under optimal analytic conditions the EDIM method is technically stable, reproducible and transferable. Biological variation over time needs further assessment in future work. 


\section{Introduction}

Current diagnostic serum assays for assessing tumour markers lack sensitivity. Their use is mostly confined to follow-up strategies, and they are always applied in combination with other diagnostic techniques ${ }^{1}$.

Recently, both Herwig et al. and our own group explored the possibility of using macrophages from peripheral blood samples to detect tumour markers in patients with proven cancer of the prostate or colon ${ }^{2,3}$. This flowcytometry-based technique enables assessment of internalized tumour-related material in activated tissue associated macrophages, using multi-color fluorochrome labelling. Both the sensitivity and specificity of this approach were better than those of regular serum assays ${ }^{3,4}$. Technical validation of this new epitope detection assay was required to assess its possible position in future clinical applications ${ }^{5,6}$. When technical validation and optimization is completed, a next step will be assessment of the test reference range in larger patient populations. To our knowledge, hardly any literature describes validation strategies for flow cytometrical detection of intracellular tumourmarkers (TM), in contrast to serum tumour marker assays ${ }^{7-9}$. To that end, a validation approach similar to clinical chemical assays seemed warranted. This paper describes validation experiments in the pre-analytic and analytic phases for the epitope detection in macrophages (EDIM)-assay. The post-analytic phase (part 1: resultpresentation, part 2: interpretation and comparison with other clinical or laboratory data and part 3: using the data in managing of disease) is standardized for part 1 and $2^{3,4}$. Part 3 is not subject of investigation in this validation-project.

\section{Materials and methods}

\subsection{Experiment overview}

After obtaining medical ethical approval and informed consent, a total group of 222 CRC in- and outpatients (35-84 years) and 23 healthy volunteers (49-58 years) were included. The total number of CRC-patients was included in subsets from 2008 to 2010; each subset was intended for a different validation part. Per subject, three EDTA blood samples were drawn in a single draw per subset. All blood samples were prepared and measured according to standard operating 
procedure (SOP) as described earlier ${ }^{3}$. The total SOP consists of three parts: the pre-analytic, the analytic and the post-analytic phase SOP.

The pre-analytic phase SOP describes the sample preparation performed using CD14APC and CD16FITC-labeling (Becton Dickinson (BD), San Jose, USA) for cell characterization based on cell surface bound epitopes. This was followed by a Fix\&Perm-strategy for labelling of epitopes intracellularly. For this purpose highly specific non-labeled primary antibodies directed against different $\mathrm{TM}^{10-13}$ were biotinylated and made detectable by a conjugation step using streptavidinrPE (BD) fluorochrome labelling. The TM assessed were Carcinoembryonic antigen (CEA, clone II-7, DAKO, Glostrup, Denmark), Transketolase-like 1 (TKTL1, R-Biopharm, Darmstadt, Germany), Cytokeratin-18 (CK 8/18, CAM5.2, BD) and M30 (M30-CytoDEATH-biotin, Peviva, Bromma, Sweden).

The analytic phase consists of the actual measurement on the flow-cytometer(s). The post-analytic phase SOP describes a series of parental gating steps. Briefly, mononuclear cells are selected based on their characteristic Forward (FSC) and Side Scatter (SSC) properties. From this population activated macrophages were selected in two steps based on CD14 and CD16 positivity. Using this CD14+CD16+ population, the fraction tumour marker positive activated macrophages were selected based on the intracellular PE-labeled tumour marker expression (Figure 5.1).

\subsection{Instrument details}

The flow cytometers used in this validation study were the FACScanto I and FACScanto II (BD). Both machines were stationed in the same hospital, but in separate departments. Flow cytometer setup of both FACScanto machines was as described in earlier studies ${ }^{3}$ : excitation of the cells was performed by a 488 $\mathrm{nm}$, air-cooled solid-state argon laser followed by a $17 \mathrm{~mW} 633 \mathrm{~nm}$ helium/neon laser. A 530-nm band pass filter enabled FITC-fluorescence detection, a $575 \mathrm{~nm}$ \pm 13 (mean \pm SD) band pass filter provided PE-fluorescence detection and APCfluorescence was assessed by using a $660 \mathrm{~nm} \pm 10$ (mean \pm SD) band pass filter. The second machine was set up and calibrated exactly like the first using standard seven-colour set-up beads (BD). Automatic compensation settings were obtained by using the FACSDiva software (BD). The SSC-settings were adjusted likewise on both machines, also assuring optimal capture range for high cell-complexity. On both machines, identical analysis templates were used, and the settings for FSC and SSC were chosen to enable optimal gating possibilities ${ }^{3}$. 

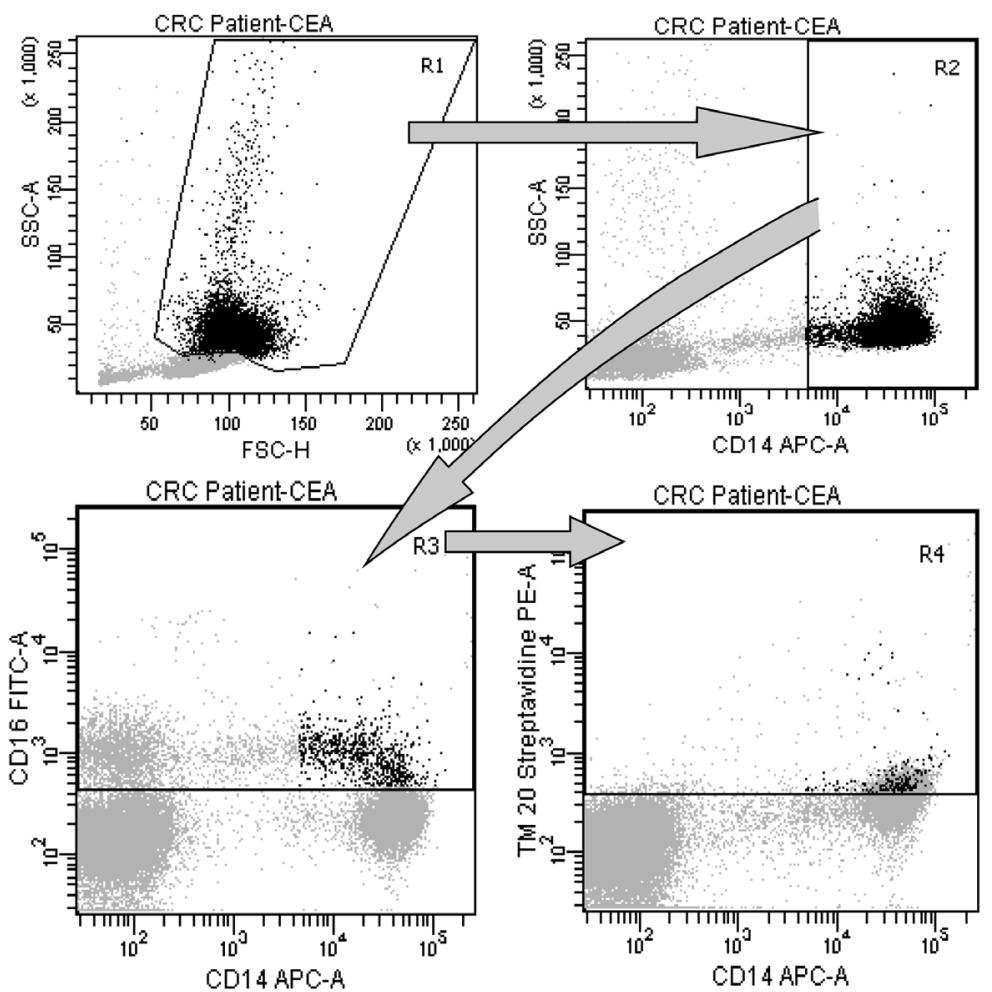

Tube: CEA

Population

\#Events \%Parent \%Total

$44,171 \# 100.0$

$\begin{array}{lrr}10,324 & 23.4 & 23.4\end{array}$

$\square R 2$
$\square R 3$

$8,664 \quad 83.9 \quad 19.6$

$800 \quad 9.2 \quad 1.8$

$211 \quad 26.4 \quad 0.5$

Figure 5.1 Parental gating strategy steps (R1 to R4-gates) for epitope detection in macrophages (EDIM): In the FSC/SSC-plot (top-left) the mononuclear cells are selected based on higher cell-size and complexity than lymphocytes and RBC. From this subpopulation the CD14+ monocytes are selected in the CD14/SSC-plot (top-right) using a fixed cut-off for CD14-positivity of above 5000. In the CD14/CD16 plot (lower-left), using the negative control (mFMO), the CD14+CD16+ activated macrophages are selected from de CD14+ population from the $2^{\text {nd }}$ plot. Again using the mFMO in the CD14/Epitope-rPE plot (lower-right) the CD14+CD16+epitopePE+ cells are selected from the CD14+CD16+ population identified in the $3^{\text {rd }}$ plot. In this experiment the epitope was a tumour-marker used for tumour-marker detection in a colorectal cancer patient, therefore the term " CRC-Patient-CEA" is used in the title above the plots. 


\subsection{Data analysis details}

FACSDiva software v6.1.2 (BD) was used for analysis of flow cytometric data files. MS Access 2003 SP2 (Microsoft Corporation, Redmond, USA) was used for data-storage, IBM SPSS-statistics 19 for mac (IBM, Armonk, USA) and Graph-pad Prism v5.0 (GraphPad Software, La Jolla, USA) were used for statistical analysis and creating graphs. In the sample stability assessment the Median Fluorescence Intensity (MdnFI) was chosen to assess epitope degradation because it is presumed to be the most robust (Shapiro and Roederer, 2008). Throughout the rest of the experiments the fraction CD14+/CD16+/EDIM+ monocytes e.g. CEA-IM, TKTL1-IM, CK-IM or M30-IM is the primary outcome measure of the EDIM-assay; further referred to as outcome EDIM-assay. Intra-class correlation coefficient (ICC), of which a value of 1.0 indicates perfect agreement, was used to assess inter-assay, intra-assay, transferability and intra-individual variations.

\subsection{Pre-analytic investigation phase details}

\subsubsection{Sample stability assessment}

Sample stability over time from venipuncture to start of preparation was assessed by storing multiple freshly drawn blood samples of 5 CRC-patients at room temperature (RT) and preparing them at time-points 0, 6 and 24 hours. Median fluorescence intensity is preferred for analysis using a logarithmic scaling $^{14}$.

\subsubsection{Biological variation}

Biological variation was assessed by repeatedly measuring samples from the same healthy person(s) over a number of weeks and months. Firstly, we assessed the variability in 6 persons during four weeks with two blood draws per week, providing 8 consecutive outcomes in a relatively short time. Secondly, we assessed the outcome-variability in (the same) six persons 10 times during 40 weeks. Combined with the measurements of the first 4 weeks, a 44-week measurement window is created. Besides biological variation inter-assay variability over a longer period is automatically assessed. All variables except time-point of venipuncture were kept static over this period. 


\subsection{Analytic investigation phase details}

\subsubsection{Intra-assay variability}

The intra-assay variability was tested by repeated measurements of the same sample on a FACS-canto I flow cytometer (BD). 90 CRC-patient samples were measured two consecutive times.

\subsubsection{Inter-assay variability}

Inter-assay variability was first assessed by preparing two different samples of the same patient, independently of each other, followed by measurement on one single flow cytometer. A single sample, prepared out of $3 \mathrm{ml}$ EDTA whole blood, is able to provide 2 to 3 consecutive measurements. Therefore we were restricted to 2 measurements per sample.

\subsubsection{Transferability}

The transferability of the EDIM-assay from one flow cytometer system to another comparable system was assessed by comparing EDIM-assay outcomes. A set of samples was measured on two flow cytometers (BD) (a FACS-canto I and a FACS-canto II) within two hours of each other. The machines were stationed in separate laboratories; different operators performed the flowcytometric analysis.

\section{Results}

\subsection{Pre-analytic investigation phase}

\subsubsection{Sample stability assessment}

Five tubes per single blood sample were analyzed using the EDIM-assay. The graphs show increase in cell size and diminishing of cell complexity over time. The expression of CD14, CD16 and EDIM changes, showing a general increase in the spread for CD16 and EDIM and an increase followed by a decrease of spread for CD14 (Figure 5.2). This variation did not interfere with the gating strategy. Statistical analysis is not feasible in a low number of measurements.

\subsubsection{Biological variation in time of CEA-IM (within healthy persons)}

Figure 5.3 shows the mean \pm SD of the fraction (\%parent) CD14+/CD16+/EDIM+ monocytes using the CEA-antibody (CEA-IM) outcomes during a period of 308 days in 6 healthy persons. 
All Mononuc fsc median

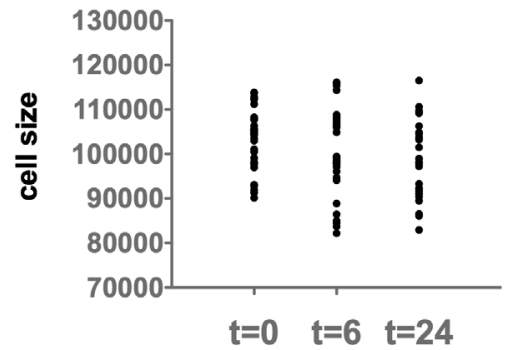

Hours from venipuncture

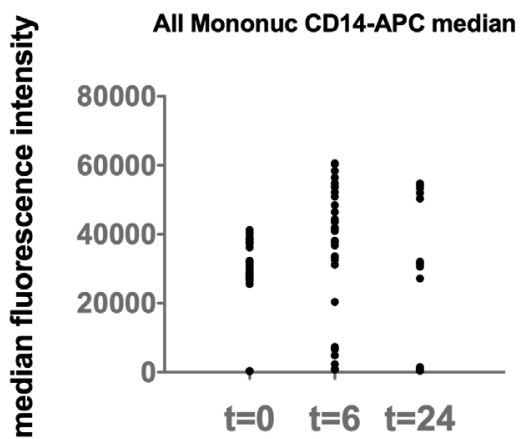

Hours from venipuncture

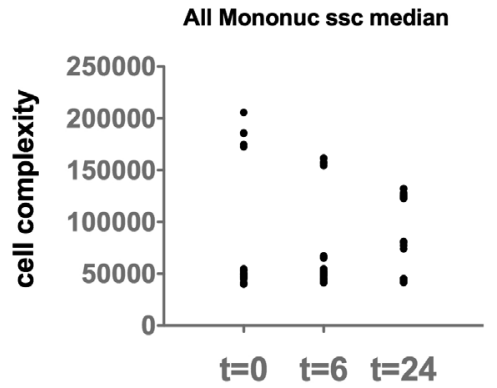

Hours from venipuncture

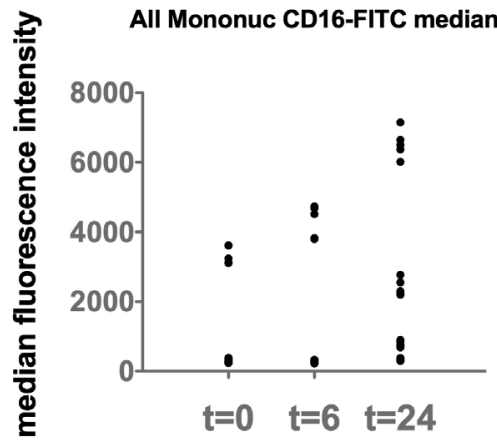

Hours from venipuncture

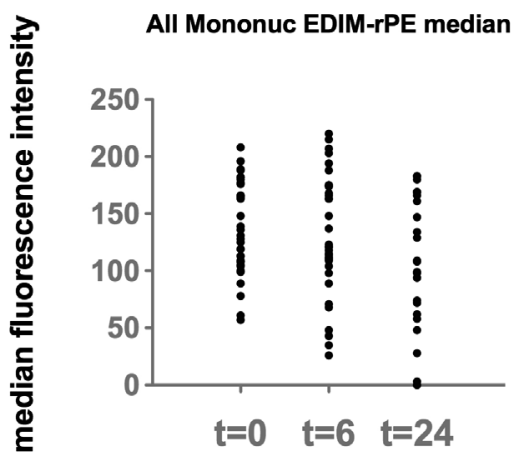

\section{Hours from venipuncture}

Figure 5.2 Sample stability assessment investigating stability of cell characteristics and mononuclear cell antibody binding sites in 5 CRC-patients. Samples were stored at room temperature on the bench and assessed at zero, six and twenty-four hours after venipuncture. 
- 6 persons mean $\pm \mathrm{SD}$

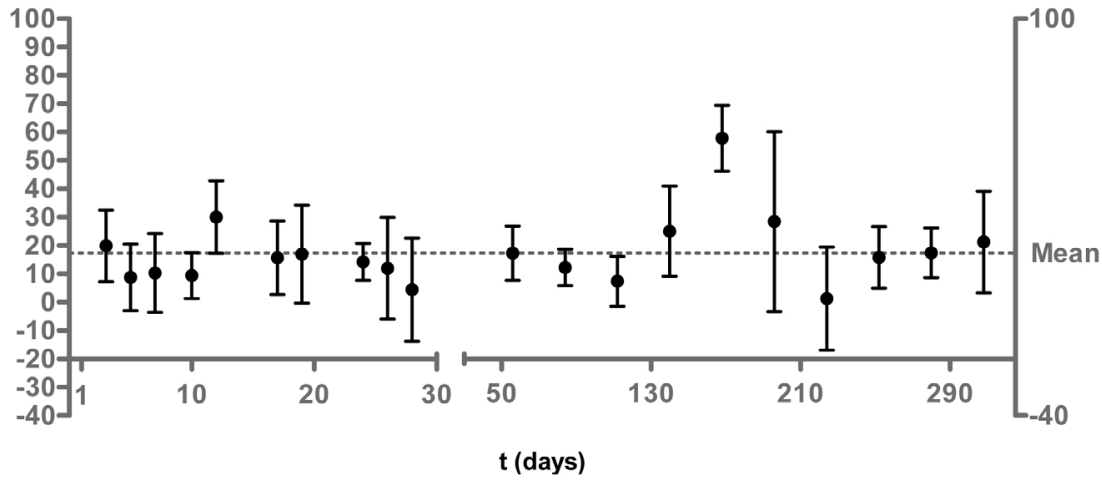

Figure 5.3 Combination of biological and inter-assay variation; showing 8 measurements over a period of four weeks, combined with 12 measurements over an additional period of 10 months of the fraction (\%parent) CEA-containing activated macrophages (CEA-IM) using the EDIM-method. The group mean \pm SD is $17.28 \pm 12.11$.

\subsection{Analytic investigation phase}

\subsubsection{Intra-assay variability}

90 single blood samples of CRC patients were drawn. 90 series of 5 analysis tubes (CEA, TKTL1, M30 and CK) and a negative control (FMO) were prepared (450 analysis tubes). All tubes were measured twice on the same machine (resulting in 450 data file-pairs). In 57 pairs, one or both tubes were disqualified due to technical failures (mostly inadequate event-numbers in either or both tubes; adequate event-numbers were defined as a minimum of 30.000 mononuclear cell events) and excluded, leaving 393 pairs for evaluation. The ICC of CEA-IM was: 0.902; of TKTL1-IM: 0.933; of CK-IM: 0.873 and of M30-IM: 0.652. The FMO itself showed an ICC of 0.836 (Figure 5.4).

\subsubsection{Inter assay variability}

Twenty-nine double blood samples from 29 CRC patients were used for interassay variability assessment. We prepared 145 pairs of analysis tubes $(29 \times 2$ series $x 5$ analysis tubes (CEA, TKTL1, M30 CK and FMO). In 7 pairs, one or both tubes were disqualified due to technical failures (mostly inadequate eventnumbers in either or both tubes; adequate event-numbers were defined as a minimum of 30.000 mononuclear cell events) and excluded, leaving 138 pairs for 
evaluation. The ICC of CEA-IM was: 0.653; of TKTL1-IM: 0.539; of CK-IM: 0.479 and of M30-IM: 0.391.The FMO had an ICC of 0.847 (Figure 5.5).

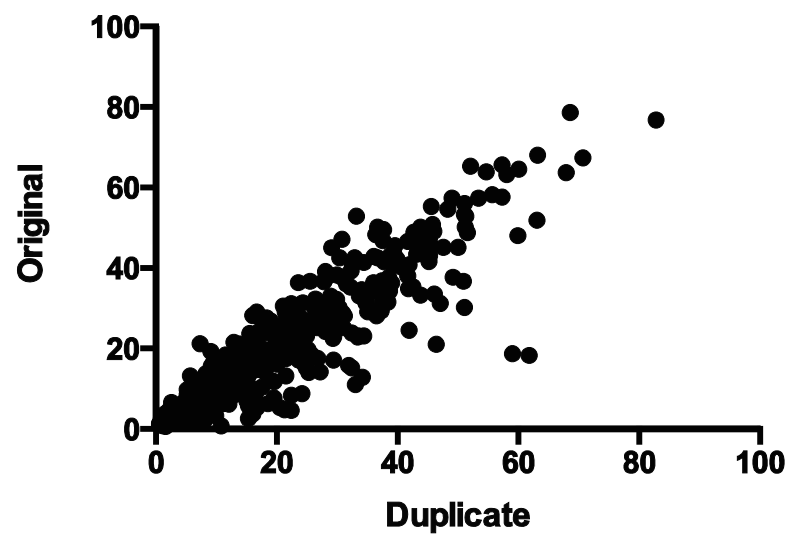

Figure 5.4 Agreement of duplicate EDIM Measurements: Using the EDIM method the reproducibility of duplicate tumourmarker measurements of all including CEA-IM is graphically shown $(n=393)$. The positioning on the 45-degree axis shows good agreement $(I C C=0,652-0,933)$ of both the original and duplicate measurement.

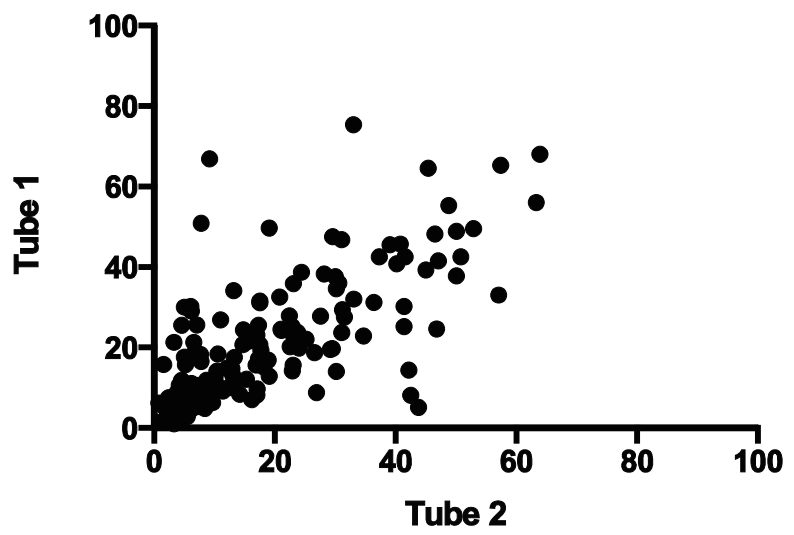

Figure 5.5 Agreement of double EDIM Measurements: Using the EDIM-method the reproducibility of all double tumourmarker-epitope measurements ( $n=138$ pairs) is graphically shown. The positioning on the 45-degree axis shows moderate to good agreement (ICC $=0,391-0,847)$ of both the first and second sample. These ICC's show less agreement than the intra-assay variability assessment. 


\subsubsection{Transferability}

Two series of duple samples of CRC patients were drawn, prepared, measured and analyzed on two different flow cytometers. In the first series 150 data files were acquired, the second series provided 120 data files. The outcomes showed excellent reproducibility between analyzers in both series. ICC of CEA-IM in series 1 was: 0.988 , and in series 2: 0.975 . ICC of TKTL1-IM in series 1 was: 0.987 and in series 2: 0.989 . ICC of M30-IM in series 1 was: 0.984 and in series 2: 0.986 (Figure 5.6 ).

\section{Agreement of flowcytometer data-pairs}

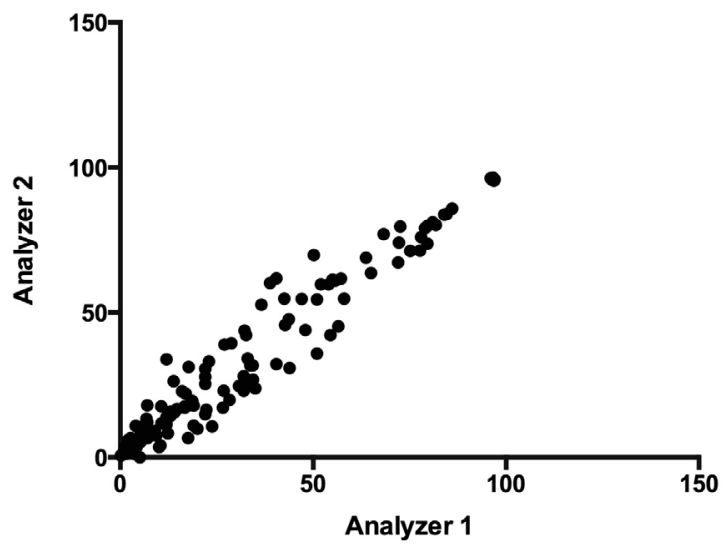

Figure 5.6 Agreement of flow cytometer data pairs: Reproducibility of 2 series of duplicate samples (series 1: $n=150$, series $2: n=120$ ) on two different flow cytometers measuring the epitopes CEA, TKTL1 and M30. All measurement-couples appear on or near the 45degree axis. Sub-zero outcomes originate from correction of assay-outcome for controloutcome, caused by assay-values not being high enough to overcome backgroundvalues (corrected for control negative value-range: -0.2 to -8.0$)$. These have all $(n=21)$ been re-corrected to a value of 0 . All duplicate measurements show excellent ICC's $(0,975-0,989)$, meaning the assay is transferable between analyzers.

\section{Discussion}

According to Sturgeon et al. of the European Group on Tumor Markers (EGTM), the pre-analytic and analytic phases of investigating an assay should be standard for serum tumour marker assays ${ }^{15,16}$. It seems logical to apply their approach to our assay. Reproducibility is considered good when intra-assay variability is $<5 \% \mathrm{CV}$ and inter-assay variability is $<10 \% \mathrm{CV}$ in automated assays. 
However, these values of intra-assay or inter-assay variability are often unachievable in experimental assays that are performed manually ${ }^{15}$.

Our group published pilot-results on epitope detection in macrophages in 2008 and $2009^{3,4}$ showing the ability to distinguish healthy controls from prostate and colorectal cancer patients using PSA-IM and CEA-IM respectively. Provided optimal technical analytical conditions are ensured, the EDIM-method is stable, reproducible and transferable, yet the pre-analytic (non-technical) phase requires attention. The assessments of sample stability and biological variation over time have only provided visual results, which have not yet been supported by statistical analysis in our group due to small numbers. The group of Feyen et al on the other hand have proven good reproducibility in co-operation with various laboratories (CV's 4.9-9.1\%) ${ }^{5}$.

The sample stability study showed changes in the binding capacity of cell surface directed antibody-fluorochrome-conjugates (CD14-APC and CD16FITC) on activated macrophages within 24 hours after venipuncture. Besides these observations, the cell size and complexity changes during time on the bench were also recorded. The observed variability within the five subjects may be due to several reasons: external antibody binding could be nonspecific and or there could be alterations in binding capacity of the binding sites. Both hypotheses may be consistent with commonly known changes observed in cell size and complexity during cell degeneration.

The parental gating strategy based on CD14 and CD16-labeling remained intact during analysis, retaining the ability to correctly characterize the cell population of interest e.g. activated macrophages. The observation of more or less stable internal cell staining within twenty-four hours suggests that intracellular staining is less sensitive to cell degradation. We suggest that this is due to capturing of the stained tumour-related material inside phagosomes protecting against external influences. This is in accordance with earlier observations of internalized PSA and CEA in macrophages using cytospins in combination with immunocytochemistry, which showed granular staining and loss of integrity of the cell membrane ${ }^{3,4}$. Considering the outcomes of the sample stability test above, starting preparation is recommended within 6-8 hours after venipuncture. In our validation studies, $91 \%$ of all samples were not only prepared, but also analyzed within eight hours.

The second pre-analytic aspect, biological variation, showed that the baseline mean value of the fraction of CEA-IM in six healthy persons over 44 weeks was 
$17.28 \%(S D=12.11)$. Due to a pre-analytic error, which was noted in the laboratory journal, an outlier occurred in all persons at 168 days that is clearly distinguishable in the graph. The variation that was found is not surprising when considering that leucocyte levels in peripheral blood samples of healthy persons vary between $8.9 \% \mathrm{CV}$ and $15.7 \% \mathrm{CV}$ within subjects, to $34.6 \% \mathrm{CV}$ between subjects ${ }^{17}$. Baseline levels per individual must be established before interpreting solitary assay-outcomes, which is in accordance with the current best practice of serum tumour marker assays. An increase in serum tumour marker levels compared with a baseline of more than $25-30 \%$ that occurs twice in a row is considered clinically relevant in $\mathrm{CRC}^{7,18}$.

During the 44-week assessment a problem occurred due to changes in LOTnumbers of Streptavidin-rPE, leading to shifts in fluorescence staining, and ultimately also in different outcomes in measurements, which were correctable by titration. This underlines the high impact of lot-to-lot variation in similarity with conventional serum tumour marker assays.

At the start of the validation process of this new assay, small adaptations to the original protocol were made ${ }^{3,4}$. The stopping gate was changed to a total of 10.000 events specific for mononuclear cells, instead of a total of 30.000 random events. In addition, another type of negative control was introduced; a fluorescence-minus-one (FMO) which had been modified (mFMO). Because our assay is based on a multicolour-, streptavidin-based immunocytochemistry method control of background fluorescence is of utmost importance. The results of our previous work were based on using a regular isotype contro ${ }^{3,4}$. However, we sometimes experienced inexplicable shifts in the EDIM-negative population for rPE-staining. A possible explanation could be the presence of endogenous biotin, which led to aspecific binding of streptavidin-rPE. It is known that it is very difficult to find an isotype control that truly matches the background staining ${ }^{19}$. Another limitation of an isotype control is that they do not account for fluorescence spillover from other channels. For that reason, we chose a modified FMO. This is a sample that includes all of the antibody conjugates except the biotin-labelled EDIM- directed antibody. This sample is finally incubated with rPE-conjugated streptavidin. Because we used high-quality fluorochrome-labelled antibodies at appropriate concentrations, the background staining due to nonspecific antibody binding is minimal. The major source of background staining is due to fluorescence spillover. Because of this, the use of FMO controls is recommended ${ }^{19}$. 
In the analytic phase, the intra-assay variability showed good results, demonstrating that it is possible to re-measure the prepared sample without significant deviations in outcome. The inter-assay variability shows mild to good agreement, while agreement on intra- and inter-assay may facilitate broader deployment options of the assay. The ICC-results in both intra-assay and interassay assessments were fairly similar. The ICC's of M30-IM were lowest in both intra-assay and inter-assay assessments. This seems to be in line with recent results of our group ${ }^{20}$ in which $\mathrm{M} 30-\mathrm{IM}$ were the least common in CRC-tissueanalysis as well. The CEA-IM, TKTL1-IM and CK-IM ICC's showed results in the same range within intra-assay and also within inter-assay assessment. This indicates that reproducibility within one blood sample is good, but between two sequential blood samples the variability is higher. This is in accordance with earlier intra- and inter-assay findings in sequential analysis of serum tumour markers ${ }^{8}$ in ovary cancer. Tuxen showed intra- and inter-assay variability of serum-CEA being very dependent on analytical and intra-individual variability, with intra-individual variability up to $58 \%$. Finally, in both intra- and inter-assay assessment practically identical mFMO ICC's were recorded, proving that variation in background-signal is very low. On 2 different flow cytometers agreement within 1 sample is good. Between sample agreement within one patient, measured on two flow-cytometers can differ from significant to mild. Besides the analytical and intra-individual variability described, the variability observed can also be due to the kind of cell separation tubes that are used. Further assessment of cell separation techniques is needed to rule out possible variability issues.

Transferability was investigated by measuring each selected sample within a certain time frame on two different flow cytometers within the same institute, but in different laboratories. This provided excellent results, proving the assay performs well when the setup procedure is performed according to the SOP in another laboratory.

In the post-analytic phase earlier established gating strategies were utilized using the $\mathrm{mFMO}$ as a blank. By comparing blank to sample outcomes all outcomes were obtained ${ }^{3,4}$.

The clinical applicability of this assay-type in cancer diagnostics has been described in different publications ${ }^{6,21}$. The EDIM-test has been shown able to detect different biomarkers for the characterization of malignancy, based on the 
detection of abnormal apoptosis, proliferation and anaerobic glucose metabolism (e.g. TKTL-1). TKTL-1 expression correlates with invasive growth and metastasis $^{6,21}$. The applicability of the assay means a step forwards in detection and surveillance options for clinicians, which in turn may lead to earlier and different treatment than was available up until now.

In summary, the EDIM-assay is a technically reproducible, stable and transferable assay. This article describes the validation of an experimental assay detecting tumour markers in macrophages. This assay exploits the natural concentration provided by the immune system. Our approach was shown to be stable during analytic procedures and partly during pre-analytic procedures. 


\section{References}

1. Coy JF, Dressler D, Wilde J, Schubert P. Mutations in the transketolase-like gene TKTL1: clinical implications for neurodegenerative diseases, diabetes and cancer. Clin Lab 2005;51: 257-73.

2. Diamandis EP. Tumor markers: physiology, pathobiology, technology, and clinical applications (Washington, DC: Amer. Assoc. for Clinical Chemistry) 2002.

3. Dot D, Miró J, Fuentes-Arderiu X. Biological variation of the leukocyte differential count quantities. Scand J Clin Lab Invest 1992;52:607-11.

4. Faber TJ, Japink D, Leers MP, Sosef MN, von Meyenfeldt MF, Nap M. Activated macrophages containing tumor marker in colon carcinoma: immunohistochemical proof of a concept. Tumour Biol 2011;33:435-41.

5. Feyen O, Coy JF, Prasad V, Schierl R, Saenger J, Baum RP. EDIM-TKTL1 blood test: a noninvasive method to detect upregulated glucose metabolism in patients with malignancies. Future Oncol 2012;8:1349-59.

6. Grimm M, Schmitt S, Teriete P, Biegner T, Stenzl A, Hennenlotter J, Muhs HJ, Munz A, Nadtotschi T, et al. A biomarker based detection and characterization of carcinomas exploiting two fundamental biophysical mechanisms in mammalian cells. BMC Cancer 2013;13: 569.

7. Herwig R, Horninger W, Rehder P, Klocker H, Ramoner R, Thurnher M, Pinggera GM, Gozzi C, Konwalinka G, Bartsch G. Ability of PSA-positive circulating macrophages to detect prostate cancer. Prostate 2005;62:290-8.

8. Jansen N, Coy JF. Diagnostic use of epitope detection in monocytes blood test for early detection of colon cancer metastasis. Future Oncol 2013;9:605-9.

9. Japink D, Leers MPG, Sosef MN, Nap M. CEA in activated macrophages. New diagnostic possibilities for tumor markers in early colorectal cancer. Anticancer Res 2009;29: 3245.

10. Kievit J. Follow-up of patients with colorectal cancer: numbers needed to test and treat. Eur $J$ Cancer 2002;38:986-99.

11. Leers MP, Kolgen W, Bjorklund V, Bergman T, Tribbick G, Persson B, Bjorklund P, Ramaekers FC, Bjorklund B, et al. Immunocytochemical detection and mapping of a cytokeratin 18 neoepitope exposed during early apoptosis. J Pathol 1999;187:567-72.

12. Leers MPG, Nap M, Herwig R, Delaere K, Nauwelaers F. Circulating PSA-Containing Macrophages as a Possible Target for the Detection of Prostate Cancer. Am J Clin Pathol 2008;129:649.

13. Maecker HT, Trotter J. Flow cytometry controls, instrument setup, and the determination of positivity. Cytometry A 2006;69:1037-42.

14. Makin CA, Bobrow LG, Bodmer WF. Monoclonal antibody to cytokeratin for use in routine histopathology. J Clin Pathol 1984;37:975-83.

15. Nap M, ten Hoor KA, Fleuren GJ. Cross-reactivity with normal antigens in commercial anti-CEA sera, used for immunohistology. The need for tissue controls and absorptions. Am J Clin Pathol 1983;79:25-31.

16. Shapiro H, Roederer M. MFI: Mean, or median fluorescence intensity. MFI: Mean, or median fluorescence intensity? Forumdiscussion: https://lists.purdue.edu/pipermail/cytometry/2008February/034498.html 2008.

17. Sölétormos G, Schiøler V, Nielsen D, Skovsgaard T, Dombernowsky P. Interpretation of results for tumor markers on the basis of analytical imprecision and biological variation. Clin Chem 1993;39:2077-83.

18. Sturgeon C. Quality requirements and control: EGTM recommendations. European Group on Tumour Markers. Anticancer Res 1999;19:2791-4.

19. Sturgeon C. Practice guidelines for tumor marker use in the clinic. Clin Chem 2001;48:1151.

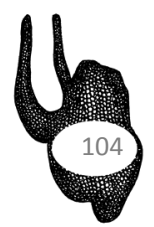


20. Sturgeon CM, Duffy MJ, Stenman UH, Lilja H, Brünner N, Chan DW, Babaian R, Bast RC, Dowell B, et al. National Academy of Clinical Biochemistry laboratory medicine practice guidelines for use of tumor markers in testicular, prostate, colorectal, breast, and ovarian cancers. Clin Chem 54, e11-e79.

21. Tuxen MK, Sölétormos G, Petersen PH, Schioler V, Dombernowsky P. Assessment of biological variation and analytical imprecision of CA 125, CEA, and TPA in relation to monitoring of ovarian cancer. Gynecol Oncol 1999;74:12-22. 



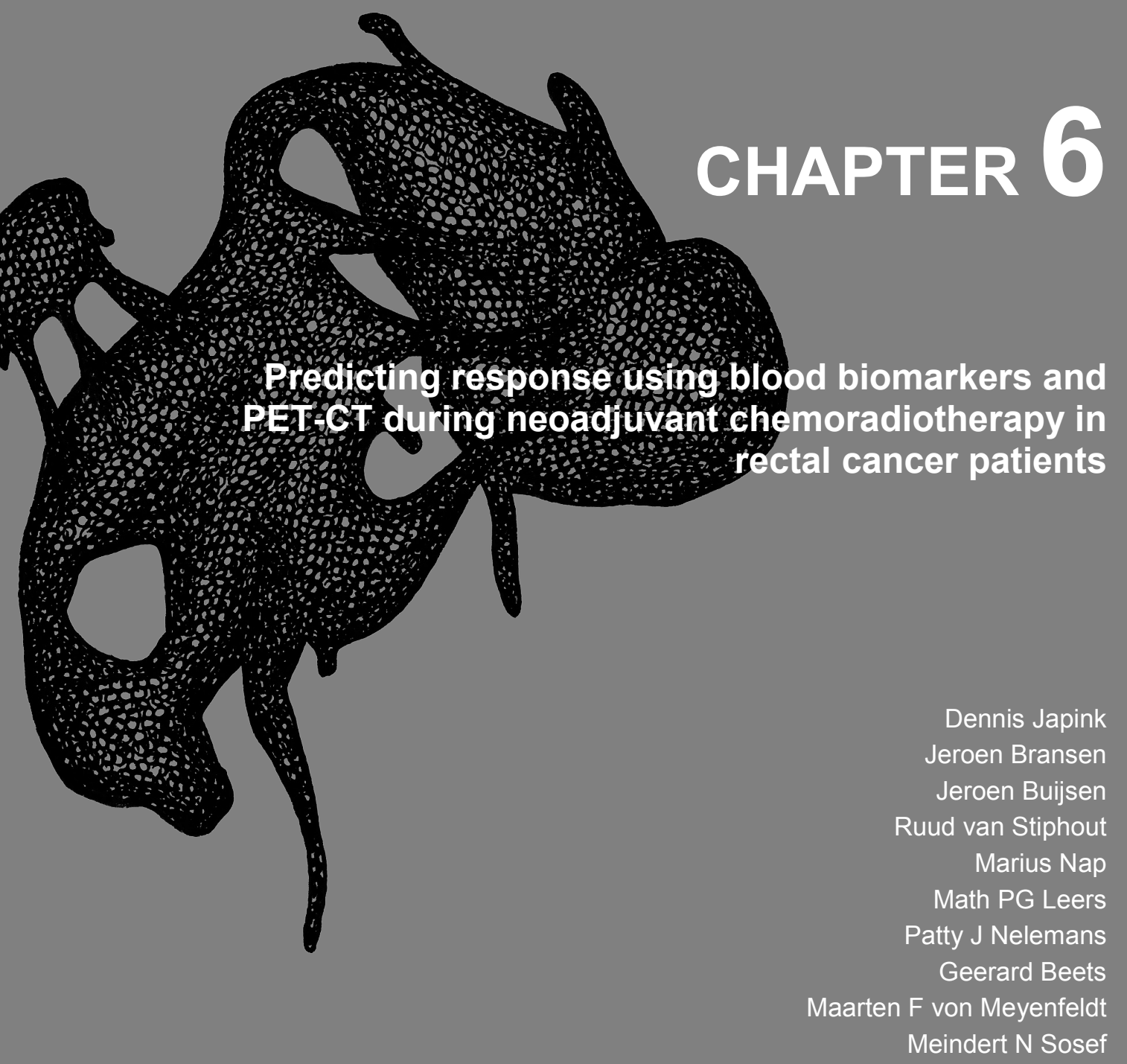

Excerpts have been submitted 


\section{Abstract}

\section{Introduction}

Response prediction and response evaluation are key objectives of the monitoring of neoadjuvant chemoradiotherapy ( $\mathrm{nCRT}$ ) in locally advanced rectal cancer (LARC) patients. These outcomes could enable early treatment alteration during the first two weeks of chemoradiotherapy. In non-responders a tool to aid the decision to perform surgery earlier is desirable. Intra and peritumoural inflammation prohibits proper response evaluation by PET-CT, and is therefore not robust enough. This study evaluates the additional value of a panel of serummarkers and experimental flow cytometrical assays to PET-CT values alone in the efficacy assessment of chemoradiotherapy. These values are assessed in relation to tumour regression (TRG) and tumour response (ypTN). The immune cell influx (INF) is investigated separately to assess reflection of biological changes during $\mathrm{nCRT}$ by blood biomarker levels and or PET-CT values.

\section{Methods}

One-hundredsixteen LARC patients were included. Peripheral blood samples were drawn and PET-CT analyses performed at baseline, 15 days and 90 days after initiation of a 50.4 Gy chemoradiotherapy protocol. Additional blood sampling was performed at day 7 in a subset of patients. In the peripheral blood samples carcinoembryonic antigen (CEA), leucocytes and c-reactive protein (CRP) were measured using routine serum assays. Flow cytometrical assays measured the fractions of monocytes, activated macrophages and CEA-containing macrophages (CEA-IM). All blood biomarkers provided time pointvalues and $\Delta$ values between time points. PET-CT measurements provided maximum standard uptake value (SUVmax), SUVmean and metabolic tumour volume (MTV) data. Results were analysed in patient subsets based on ypTN, TRG and immune cell influxscore (INF). AUCs were calculated using ROC-curve analyses to assess outcome discriminative capabilities for TRG and ypTN. Added value to existing response prediction models at $t=0, t=15$ and $t=90$ was assessed. INF was investigated to assess the biological effects of nCRT treatment during the treatment period.

\section{Results}

Of 116 included patients 94 qualified for analysis of which $23(24.5 \%)$ developed a ypTON0 and 55 (58.5\%) were good responders (ypT0-2N0). In univariate analysis serum CEA was predictive at $\mathrm{t}=0$ using TRG $1-2$ and ypTONO as outcome measure with AUC of $0.656(p=0.011)$ and AUC $0.600(p=0.09)$ respectively. MTV at $t=0$ was predictive for TRG 1-2 (AUC=0.666, $p=0.008), y p T 0 N 0 \quad(A \cup C=0.750, p=0.001)$ and ypT0-2N0 $(A \cup C=0.674, \quad p=0.005)$ respectively. Other blood biomarkers at various time 
points/periods showed trends towards predictive cut-off value 0.6. Combining PET-CT with baseline blood biomarker values increased the AUC from 0.74 to 0.75 in the first prediction model $(t=0)$ of $\mathrm{pCR}$. The second blood biomarker enhanced prediction model ( $\mathrm{t}=0-15$ days) showed an AUC of 0.72 for PET-CT alone and an AUC of 0.94 for PET + biomarkers predicting pCR. A third blood biomarker enhanced prediction model $(\mathrm{t}=0-90$ days) showed an AUC of 0.75 for PET-CT alone and 0.76 for PET-CT + biomarkers predicting $\mathrm{PCR}$. Biological changes in the tumour (and surroundings) were reflected best by the MTV. MTV was predictive for low immune-cell influx (INF 0-1) at $\mathrm{t}=0-15$ days and $t=15-90$ days $(A \cup C=0.78(p=0.002)$ and $A \cup C=0.72(p=0.078)$.

\section{Conclusion}

Serum CEA and MTV showed significant discriminating abilities on $\mathrm{t}=0$ investigating the outcome measure TRG1-2. Recently published results predicting pCR (ypTON0) outcome at $\mathrm{t}=0$ using serum CEA have now been confirmed in this different patient population investigating ypTON0 as well as TRG1-2. TRG1-2 therefore seems equal to ypTON0. Using a blood biomarker enhanced pCR-prediction model shows an AUC of 0.94 in a prediction model applied in the first 2 treatment weeks of chemoradiotherapy. Therefore, treatment alteration based on $\mathrm{pCR}$ outcome prediction in the first two treatment weeks seems feasible. Confirmation of these findings in a prospective trial using a larger cohort in another population area would be necessary to exclude study bias. 


\section{Introduction}

Neoadjuvant chemoradiotherapy ( $\mathrm{nCRT}$ ) is widely used in patients with locally advanced rectal cancer (LARC). Besides decreasing local recurrences, downsizing and downstaging can be accomplished. Complete pathological response $(\mathrm{pCR})$ is seen in $12-27 \%{ }^{1}$ and some recent studies suggest that these patients can be offered a wait-and-see-policy ${ }^{2-4}$. Good responders ( $\left.>50 \%\right)$ show reduction in both tumour size and lymph node positivity (down-staging), leading to possibilities for less invasive surgical intervention. Absence of such reactions (non-responders) could warrant earlier surgery, or exposure to higher or prolonged doses of radiation and/or chemotherapy ${ }^{5,6}$.

Evaluation of response status for treatment alteration purposes is only possible if the response status can be properly determined. One of the promising imaging modalities is (FDG) PET-CT. PET-CT pre- and post-treatment has shown $78 \%$ sensitivity (range $75-82 \%, 95 \% \mathrm{Cl}$ ) and $66 \%$ specificity (range $62-69 \%, 95 \% \mathrm{Cl}$ ) in response prediction ${ }^{1}$. Maximum standardised uptake value (SUVmax) and response index $(\mathrm{RI})$ at day 15 are stated as the most promising predicting factors ${ }^{1}$. MRI, regular CT and endorectal ultrasound (ERUS) proved to be inferior to PET-CT, but equally capable in predicting non-responders ${ }^{7,8}$. PET-CT is currently the imaging modality of choice in current clinical trials for response monitoring and prediction.

Recently it was shown that the accuracy level of an established response prediction model based on clinical parameters and FDG PET-CT-information could be enhanced using blood biomarkers ${ }^{9}$. Buijsen et al. focussed on the additional value of multiple pre-chemoradiotherapy blood biomarker values. They reported serum carcinoembryonic antigen (CEA) and osteopontin as significant predictors for $\mathrm{pCR}$ after univariate analysis. For good tumour response (ypT0-2N0), CEA, interleukin 8 (IL-8) and osteopontin were the strongest predictors. The prediction model accuracy was increased to 0.81 for pCR and to 0.78 for tumour response. However, interpretation of the results in relation to TRG and INF was not performed.

Blood biomarker variation during nCRT has had little attention up until recently. In previous studies we investigated a flow cytometrical assay which detects intracellular accumulation of tumour related material in macrophages in peripheral blood of CRC patients. Intracellular detection in macrophages of CEA (CEA-IM) attributed to early stage CRC diagnostics. Additional CRP- 
measurements effectively distinguished CRC from inflammatory bowel diseases $^{10,11}$.

Treatment result assessment using PET-CT and blood biomarkers investigating tumour response and (peritumoural) inflammation is not generally implemented and remains under investigation ${ }^{9}$. Tumour response can be assessed on the basis of the following outcome measures: tumour regression grade (TRG according to Mandard ${ }^{12}$, considered to be the gold standard) and tumour response (ypTN). The low-grade inflammation already present in colorectal cancer $(\mathrm{CRC})$ tumours and their microenvironment ${ }^{13}$ is modulated during neoadjuvant chemoradiotherapy. This results in local oxygen radical production and increased microvascular permeability, both causing tissue damage by extensive leukocyte infiltration and oedema ${ }^{14}$. Peritumoural inflammation causes interference in tumour volume-assessment using PET-CT, hindering tumour response interpretation. Peritumoural inflammation can be assessed in the pathological specimen objectifying local immune cell influx and fibrosis. The local biological effects of nCRT caused by the presence or absence of the immune cell influx have not yet been evaluated. Assessing tumour inflammation could provide more information about a possible difference between responders and non-responders based on the patient's immune system abilities to respond adequately to the nCRT. Tumour inflammation and immune cell influx can be assessed investigating a semi-quantitative 4 point score, the INF-score.

The aim of this current study is to assess values and value-variations of blood biomarkers and PET-CT parameters in relation to TRG, ypTN and INF in rectal cancer patients receiving nCRT. The ypTN will be evaluated for additional value to $\mathrm{pCR}$ prediction models ${ }^{9,15,16}$. The INF score will be assessed to investigate local biological effects caused by nCRT and immune cell influx. All parameters were assessed before nCRT started, during the $\mathrm{nCRT}$ period and during the waiting/recovery period.

\section{Materials and methods}

\subsection{Patient population}

During the period July 2008 to july 2011, 116 patients with LARC were included in the combined PET-CT and blood biomarker analysis of MAASTRO Clinic, Atrium Medical Center Parkstad and Maastricht University Medical Center.

The study was performed according to the Helsinki Declaration and was approved by the Medical Research Ethics Committee (MREC) of the Maastricht 
University Medical Center and for local application by the MREC of the Atrium hospital.

For this study, the following types of LARC were included: distal tumours with a T3- and/or N2 stage, mid- or upper rectal tumour with a circumferential resection margin prediction of $<2 \mathrm{~mm}$ and $\mathrm{T} 4$ tumours

Clinical TNM staging was performed by MRI. Patients underwent surgery 8-10 weeks after finishing nCRT. In total 22 patients were excluded for various reasons: physical complaints $(n=14)$, disease progression $(n=4)$ and refusal of venipuncture $(n=4)$.

The remaining $n C R T$ study population $(n=94)$ was treated according to a 50.4 Gy chemoradiotherapy protocol ${ }^{9}$. During nCRT peripheral blood samples (EDTA, heparin and serum) and PET-CT images were obtained on days 0, 7, 15 and 90. During the study, several patients dropped out at or between each study time point $(t=15, n=86 ; t=90, n=67)$. Reasons for withdrawal were physical complaints, disease progression, blood sampling errors or technical laboratory problems. TRG and INF-scores could not be recovered for all patients; therefore the study populations for TRG $(n=90)$ and INF $(n=59)$ consist of subgroups of the total study population of 94 patients. Finally, an additional blood sampling on $t=7$ days of a subpopulation of patients was obtained to investigate variations within the first two treatment weeks. In this subgroup the known number of ypTN-statuses was $n=34$. The TRG status was recovered in $n=33$ patients and INF-score in $n=21$ patients.

\subsection{Blood analysis}

All blood samples were analysed within 8 hours after blood withdrawal. CEA was determined in serum on a Centaur (Siemens, Germany). CRP was tested in heparin by a particle-enhanced immunoturbidimetric assay on a Roche Cobas system (Roche Diagnostics $\mathrm{GmbH}$, Mannheim, Germany). Leukocytes were determined on an automated hemocytometer system (Sysmex XE2100, Kobe Japan).

\subsubsection{Flowcytometrical analysis}

Peripheral EDTA-blood samples for flow cytometry were prepared and analysed as described in previous publications ${ }^{10,11}$. In short, peripheral blood monocytes were isolated from whole blood and relevant epitopes are labelled extra- and intracellularly. Using this technique, cell size and granularity, as well as intraand extracellular epitope presence (e.g. CEA (CEA-IM)) can be investigated. 
The fractions (\%parent) of blood monocytes, activated macrophages and CEAIM were assessed.

\subsection{PET-CT measurements}

PET-CT measurements were obtained applying the same technical PET-CT protocols as published earlier ${ }^{9,16,17}$. Patients received an intravenous FDGinjection with weight-dependent dose after 6 hours of fasting. Sixty minutes later PET-CT-scans were acquired. PET-CT-data was normalized for blood glucose levels obtained shortly before FDG-administration. Obtained PET-CT parameters were maximum standard-uptake value (SUVMax), mean standard uptake value (SUVMean) and Metabolic Tumour Volume (MTV) of the tumour. All values were obtained by an experienced PET-CT analyst.

\subsection{Histological specimen and tumour inflammation evaluation}

Tumour regression (TRG) and response (ypTN) were obtained by examining the pathology reports. If available, TRG according to Mandard was obtained besides ypTN. When TRG was absent, ypTN was used as primary outcome measure. In order to assess local tumour inflammatory response a non-validated semiquantitative 4 point scoring system was used. Immune cell infiltration (INF) was assessed by scoring immune cell infiltration and fibrosis in 4 categories according to histopathological evaluation: INF $0=$ no infiltration or fibrosis visible; INF 1=sparsely visible local reactive fibrosis; INF 2=evident fibrosis and some inflammatory cell(s)/deposits); and INF 3=finally widespread fibrosis and massive inflammatory cell influx. If descriptions were absent or incomplete, the INF-score was not determined. Standard pathology reports provided sufficient information in $62 \%$ of our study population. Finally, pooling of the results of the INF 0 and INF 1 groups into an INF Low group, along with pooling of the results of the INF 2 and INF 3 groups into an INF High group was performed. Regrouping enabled statistical analysis in equally large dichotomized groups.

\subsection{Patient group selections and endpoint selection}

Results are displayed by, and calculated in, patient groups based on the following endpoints: Pathological tumour response (ypTN; $n=94$ ), Tumor Regression Grade (TRG; $n=90$ ) and immune cell infiltration (INF; $n=59$ ). As a primary endpoint TRG was chosen because it is currently considered to be the gold standard; ypTN was a considered secondary endpoint. 


\subsection{Result presentation and statistical analysis reporting}

Paragraph 3.2 displays the results in tables and graphs with the aim of visually evaluating the changes during $\mathrm{nCRT}$ in the various patient groups. This approach enables identification of interesting variations over time in combination with statistical outcomes of all parameters that have been reported on in paragraph 3.3.

\subsection{Statistical analysis}

\subsubsection{Univariate analysis}

Statistical analysis was performed in GraphPad Prism v 6.0 for Macintosh (GraphPad, La Jolla, CA, USA) and SPSS v 22.0 for Macintosh (IBM, Armonk, NY, USA). Statistical tests used were: ROC-curve analysis to assess AUCvalues of the calculated predicted probabilities, $95 \%$ confidence intervals and accompanying $p$-values. Confidence intervals with lower-boundaries of 0.500 and above were considered significant ${ }^{18}$, reflecting in subsequent $p$-values equal to or below 0.05 . Calculations of the AUC-values were made at the baseline time-point $(\mathrm{t}=0)$, and value-changes (or delta) within each time period, defined as $\mathrm{t}=0-7$ days, $\mathrm{t}=7-15$ days, $\mathrm{t}=0-15$ days and $\mathrm{t}=15-90$ days.

\subsubsection{Completing biomarker values for prediction model evaluation.}

Missing blood biomarker-values were completed using expectation-maximation imputation with a kNN imputation algorithm. This imputation method was only applied for investigating additional value to the existing prediction model, not in above described univariate analyses. Adding all parameters by Mathlab, release 2010b (The Mathworks, Natick, MA) to prediction models was used to assess possible increase in predicting capacity for ypTONO (pCR). For baseline interpretation, a PET0 model was built of cT-stage, cN-stage, max diameter of tumour on PET-CT combined with the SUVmean at $\mathrm{t}=0^{16}$. The established parameters of the existing PET15 prediction model, cT-stage, cN-stage and maximum diameter of the tumour on PET-CT were combined with $\triangle$ SUVmean $\mathrm{t}=0-15$ days $^{17}$. For the PET90 model the combined parameters consisted of tumour length, SUVMax, $\triangle$ SUVMax 0-90 days ${ }^{16}$. A ROC-analysis with AUC calculation was performed after bootstrapping $(n=1000)$ to approximate the true AUC as in previous studies ${ }^{9,16,17}$. 


\section{Results}

\subsection{Patient characteristics}

In Table 6.1 the characteristics of the total patient population and subpopulation at $t=7$ are shown. The age in both groups is comparable; the subgroup consists of slightly fewer men. In the subgroup, the cT3 and the cN1 stage are relatively more prevalent than in the total patient group. The subpopulation therefore consists of patients with more invasive tumours with less extensive nodal involvement.

Table 6.1 Patient characteristics nCRT group.

\begin{tabular}{lcccc} 
Patient characteristics & \multicolumn{2}{c}{$\begin{array}{c}\text { Total population } \\
(\mathrm{n}=94) \\
\%\end{array}$} & $\begin{array}{c}\text { Subpopulation at } \mathrm{t}=7(\mathrm{n}=34) \\
\mathrm{n}\end{array}$ \\
$\begin{array}{l}\text { Age } \\
\quad \text { Median }\end{array}$ & 65.9 & & & \\
$\quad$ Range & $34.4-88.0$ & & $34.4-88.0$ & \\
Gender & & & & \\
$\quad$ Male & 71 & 75.5 & 24 & 70.6 \\
$\quad$ Female & 23 & 24.5 & 10 & 29.4 \\
Clinical tumour stage (CT) & & & & \\
2 & 10 & 10.6 & 2 & 5.9 \\
3 & 59 & 62.8 & 28 & 82.4 \\
4 & 25 & 26.6 & 4 & 11.8 \\
Clinical nodal stage (cN) & & & & \\
0 & 17 & 18.1 & 8 & 23.5 \\
1 & 36 & 38.3 & 15 & 44.1 \\
2 & 41 & 43.6 & 11 & 32.4
\end{tabular}

\subsection{Blood biomarkers and PET-CT before, during and after nCRT}

Tables 6.2, 6.3, 6.4 and 6.5 show the actual means \pm SD at each point in time per outcome measure in the TRG, ypTN and INF outcome group-comparisons respectively. On $\mathrm{t}=7$ only a subgroup was investigated for blood biomarker values, no PET-values were obtained. Accompanying Figures 6.1A-C, 6.2A-C, 6.3A-C and $6.4 \mathrm{~A}-\mathrm{C}$ visually demonstrate the variation in serum $\mathrm{CEA}$, serum $\mathrm{CRP}$ and serum leucocytes. Figures 6.1D-F, 6.2D-F, 6.3D-F and 6.4D-F show the variation in the flow cytometrical measured fractions of monocytes, activated macrophages and CEA-IM. Figures 6.1G-I, 6.2G-I, 6.3G-I and 6.4G-I show SUVmean, SUVmax and MTV value variation. The subgroup analyses between $\mathrm{t}=0$ and $\mathrm{t}=7$, at $\mathrm{t}=7$ and between $\mathrm{t}=7$ and $\mathrm{t}=15$ in all outcome measures show low patient numbers of below 10 patients and should be interpreted as such. The 
discriminative power of all parameters or changes thereof are explored below in section 3.3.

\subsubsection{Tumour regression grade (TRG)}

The baseline CEA value in good responders is markedly lower than in bad responders. Measurements on $t=7$ show an interesting separation between good and bad responders in CEA, CRP and \%parent Monocytes. This could be real, or a result of the selection effect of the subgroup selection.
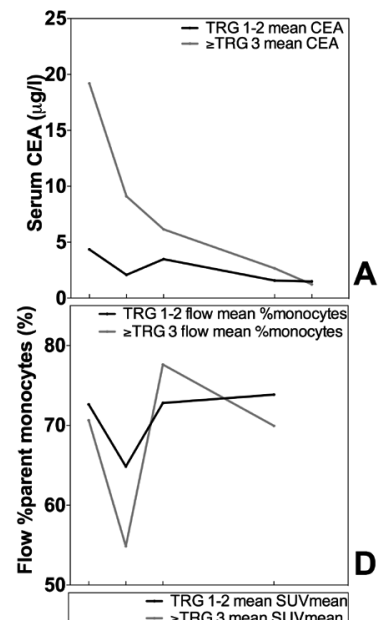

A
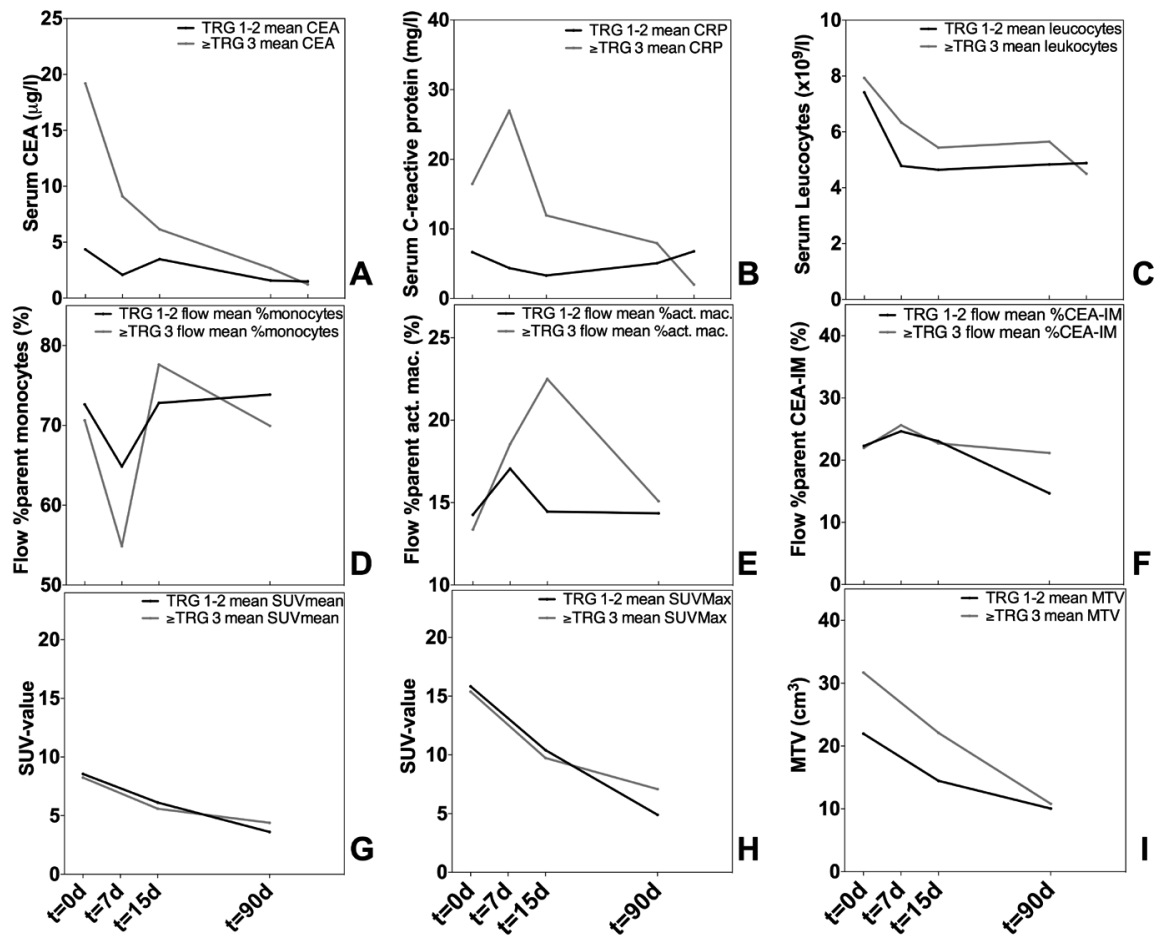

Figure 6.1 A-I: Figure 1 shows the influence of neoadjuvant treatment on serum, flow cytometrical and PET-CT parameters considering TRG as the final outcome measure during the whole $\mathrm{nCRT}$ treatment period.

\subsubsection{Pathological response (ypTN)}

Patients were divided firstly by complete and incomplete pathological response, pCR (ypTON0) and non-pCR (ypT1-4NallMall) respectively (Table 6.3 and Figure 6.2). Secondly they were divided by good and bad response, ypT0-2N0 and ypT1-4NallMall (Table 6.4 and Figure 6.3). 

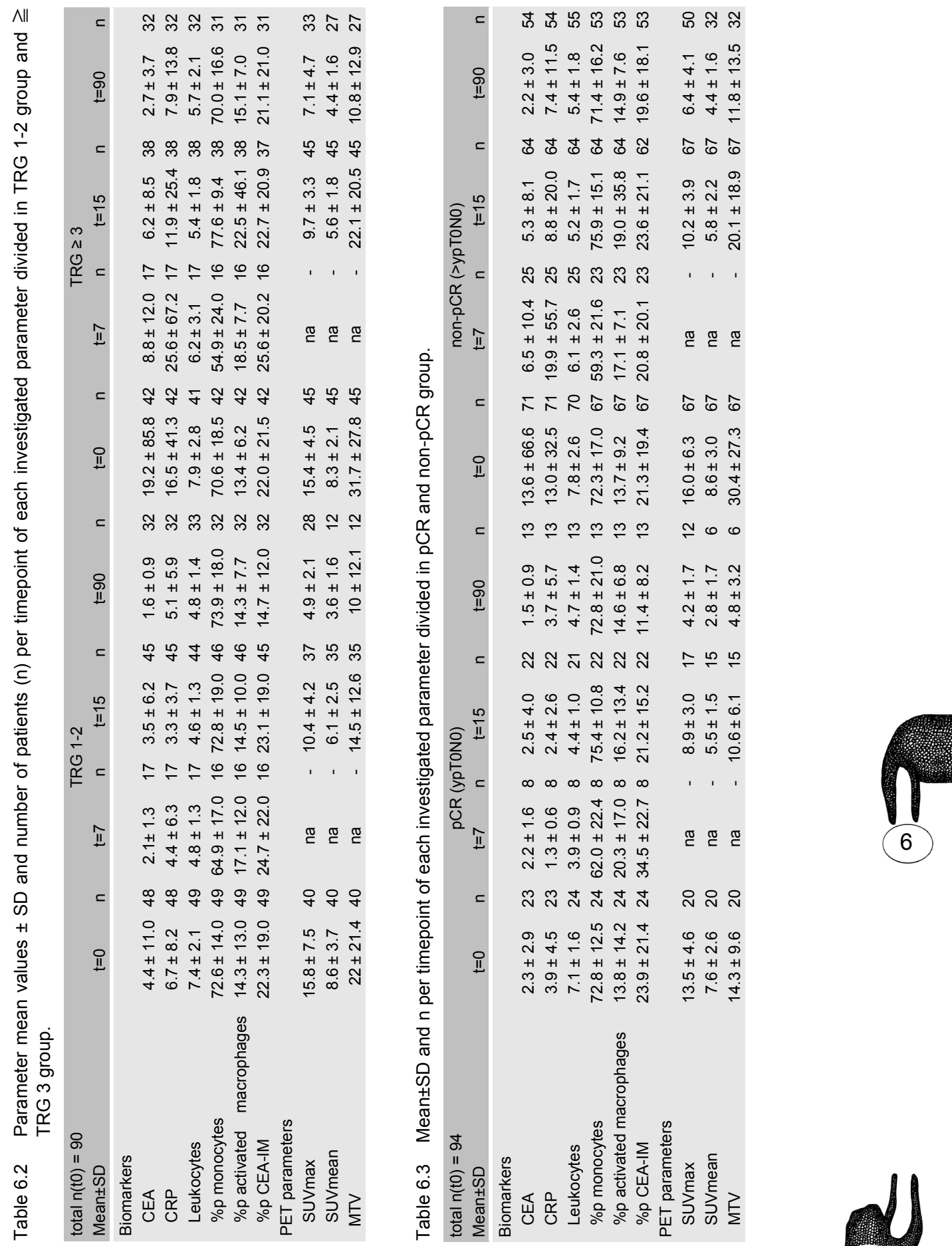


\subsubsection{Complete (ypTONO) vs. incomplete response >ypTONO}

Serum CEA shows a decrease during the 90 day period. CRP shows an initial decrease in the $\mathrm{pCR}$ group, while it increases in the non-pCR group. A decrease in leucocytes in non-pCR group between $t=0$ and $t=90$ is shown, the $p C R$ group shows a steep decrease and a subsequent small increase. The fractions of activated macrophages and \%CEA-IM in the $\mathrm{PCR}$ group show some different responses between $\mathrm{pCR}$ and non-pCR at day 7 , although sample size is small. There is a difference in MTV between $\mathrm{pCR}$ and non-pCR groups from the start throughout the neoadjuvant treatment period. SUV-values decrease during treatment in both groups (Table 6.3 and Figure 6.2).
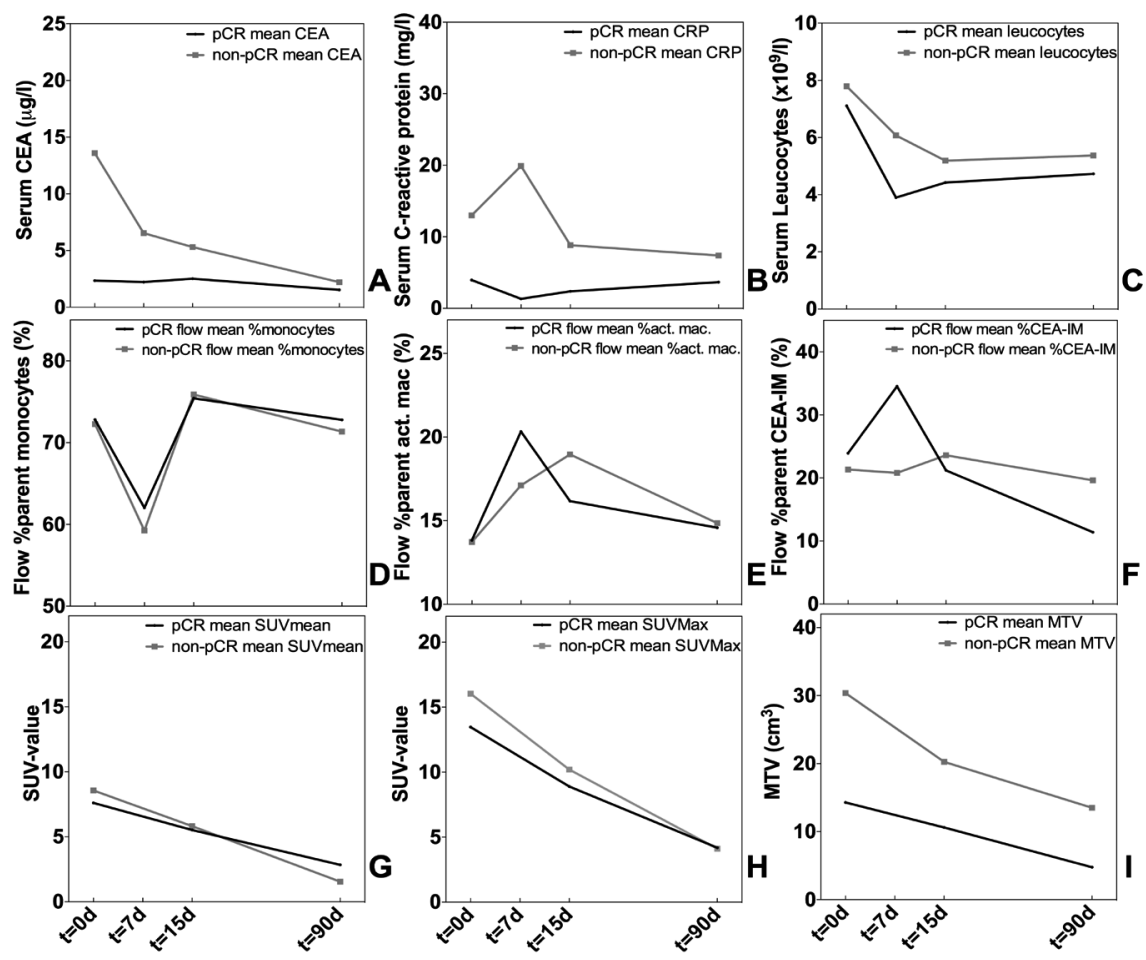

Figure 6.2 A-l: Figure 6.2 shows the influence of neoadjuvant treatment on serum, flow cytometrical and PET-CT parameters considering pathological response (ypTN) as the final outcome measure; this first ypTN figure compares the pathological complete response group ( $\mathrm{pCR} / \mathrm{ypTON0}$ ) with the group of pathological incomplete response (non-pCR / > ypTONO) during the whole nCRT treatment period. 


\subsubsection{Good (ypT0-2N0) vs. bad response (ypT3-4NallMall)}

CEA continues to show a decrease during 90 days and CRP again shows an increase and subsequent decrease during the first fifteen days in the bad response group. A decrease in leucocytes is shown in both groups between $t=0-15$ days, whereafter both groups seem to remain stable. The fraction of monocytes shows a more pronounced decrease in the bad response group than the good responders. The fraction of activated macrophages in the good response group shows a stronger increase during $t=0-7$ days than the bad response group, but the fraction of CEA-IM is almost equal in both groups. SUVvalues again decrease during treatment in both groups (Table 6.4 and Figure 6.3). In general, the results resemble the outcomes of the complete responders vs the non-responders in paragraph 3.2.2.1.
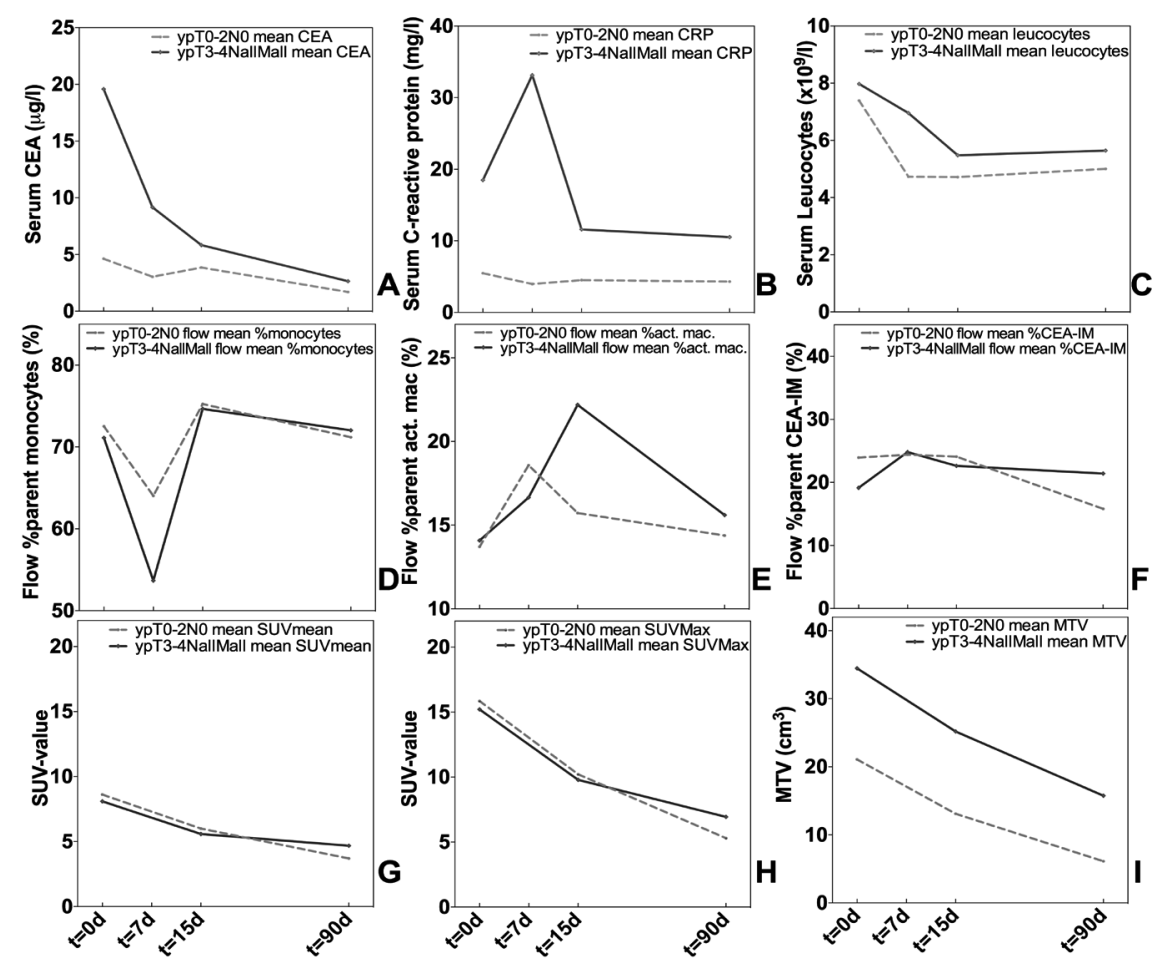

Figure $6.3 \mathrm{~A}$-I: Figure 6.3 shows the influence of neoadjuvant treatment on serum, flow cytometrical and PET-CT parameters considering pathological response (ypTN) as the outcome measure; this second ypTN figure compares the pathological good response group (ypT0-2N0) with the group of bad pathological response (ypT3-4NallMall) during the whole nCRT-treatment period. 


\subsubsection{Tumour inflammation and immune cell influx (INF)}

Patients were divided by high influx of immune cells and fibrosis (INF-High=INF $2+3$ ) vs low influx of immune cells and fibrosis (INF-Low=INF $0+1$ ) respectively, both based on pathological specimen evaluation(Table 6.5 and Figure 6.4).
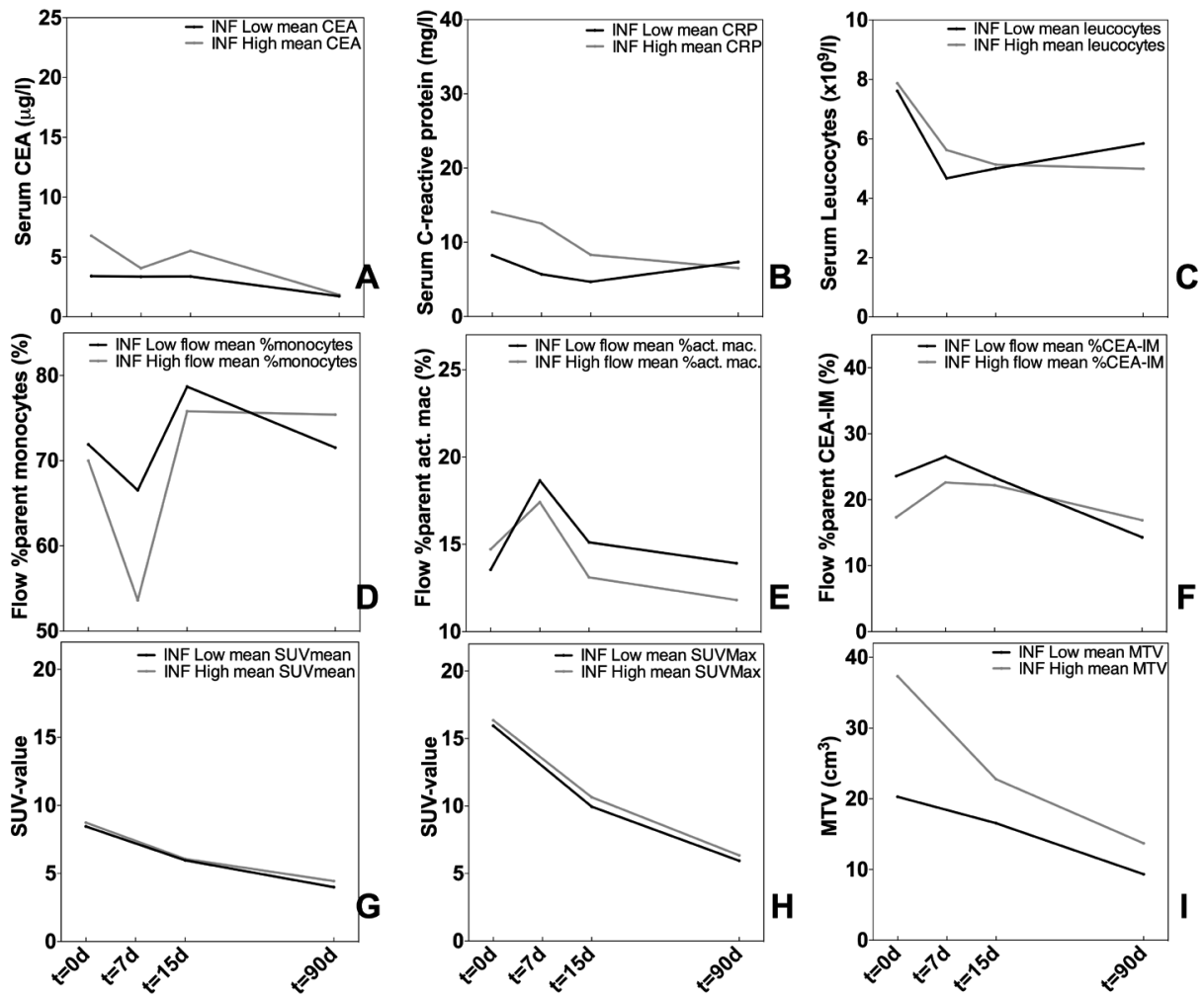

Figure $6.4 \mathrm{~A}$-I: Figure 6.4 shows the influence of neoadjuvant treatment on serum, flow cytometrical and PET-CT parameters considering influx of immune cells and fibrosis as outcome measure during the whole nCRT-treatment period.

At $t=0$ CEA and CRP show higher baseline values in the INF-High group. CEA shows only little difference between both groups during $t=0-90$ days. CRP and Leucocytes both decrease between $\mathrm{t}=0-15$ and $\mathrm{t}=0-7$ respectively. Leucocytes increase slightly after $\mathrm{t}=15$ for INF-Low but seem to remain stable for INF-High. The fraction of monocytes shows a bigger decrease in the INF-High group than the INF-Low group. The fraction of activated macrophages in both groups almost 
show equal values and patterns. The fraction of CEA-IM baseline value in the INF-High group is higher; during neoadjuvant treatment both increase until $t=7$ and start decreasing until $t=90$, where the INF-Low group decreases the most and the fastest. SUV-values decrease during treatment in both groups in similar patterns. The MTV-value of INF-High is at baseline higher than INF-Low and stays clear of the INF-Low line during the whole period. This final observation seems to be the most pronounced in this tumour inflammation assessment based on pathological immune cell influx and fibrosis.

\subsection{Predictive value (AUC) of blood biomarkers and PET-CT- values on TRG, ypTN and INF outcomes}

In tables 6-9 the predictive values of the measured blood biomarkers and PETCT values have been calculated and expresses as AUC for the outcome measures TRG, ypTN and the biological effects of nCRT using INF. Measurements on $\mathrm{t}=7$ have been additionally acquired in a subgroup, and show interesting variations during early neoadjuvant treatment. In CEA, CRP and the fraction of monocytes these variations are most pronounced (AUC-calculations for SUVmax, SUVmean and MTV were not available in this subgroup in the period $t=0-7$ days and $t=7-15$ days because no PET-CT was made on $t=7$ ).

\subsubsection{Tumour regression grade (TRG)}

Metabolic tumour volume (MTV) is a significant discriminator at $\mathrm{t}=0$ for tumour regression $(A \cup C=0.666, p=0.008$ ). The change in serum $C E A$ level between 0 and 15 days of treatment has an AUC of 0.602. In the neoadjuvant treatment period $t=0-7$ days, CRP is significantly discriminative $(A U C=0.816, p=0.002)$ for TRG 1-2; also leucocytes and the fraction of activated macrophages show AUClevels of 0.605 and 0.607 respectively. In the period $t=7-15$ days the fraction of monocytes is significantly discriminative $(A \cup C=0.737, p=0.028)$. The number of patients is lower than at other time points $(n=17)$. 
Table 6.6 Calculated AUC-values of all parameters: AUC-values of the predicted probability for TRG1-2 as positive predicted outcome combined with the $95 \% \mathrm{Cl}$ and $\mathrm{p}$-values are shown.

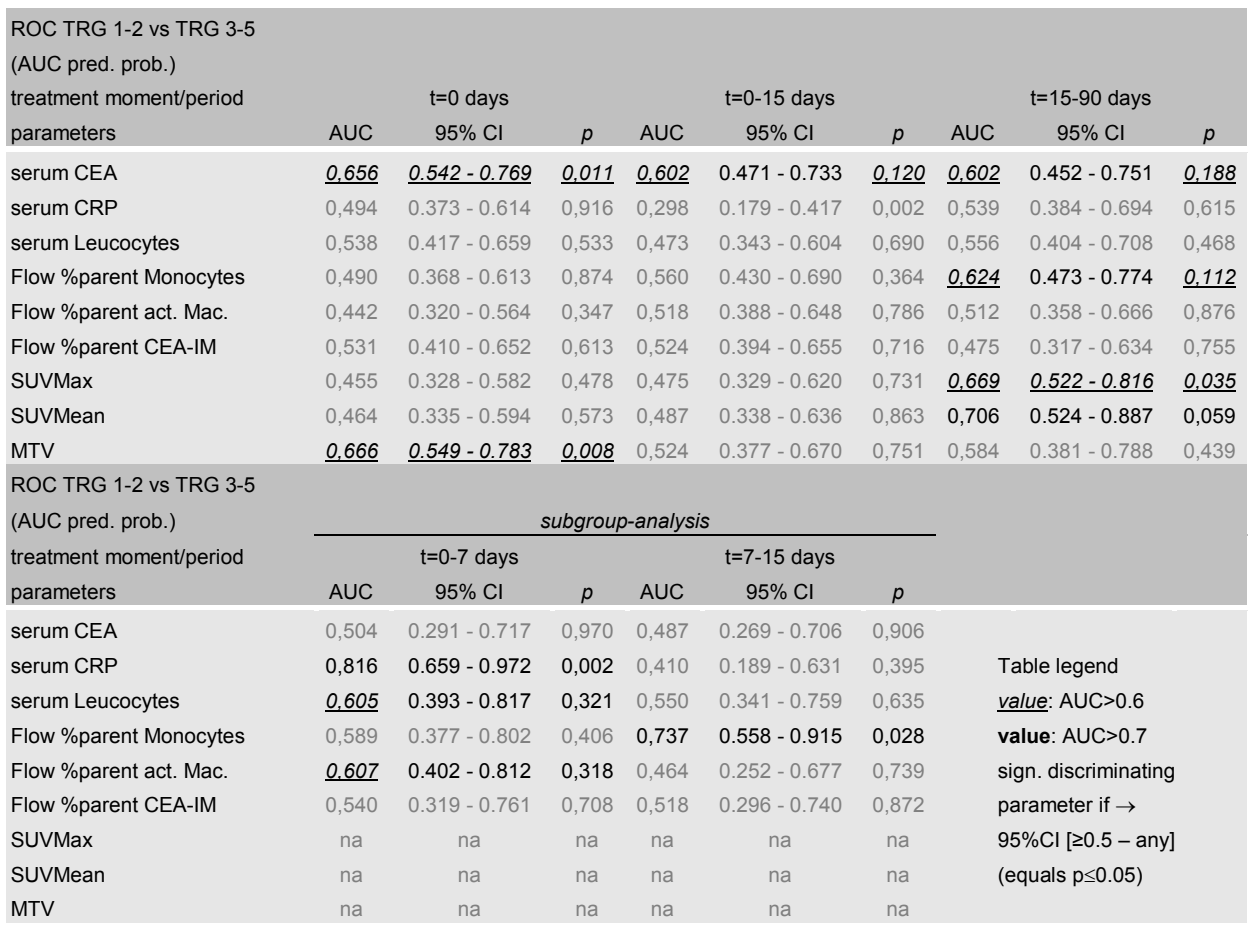

\subsubsection{Pathological response (ypTN)}

\subsubsection{1 ypTONO vs. >ypTONO}

MTV is the only significantly discriminative parameter at $t=0$ when using $p C R$ as an outcome measure. CEA, leucocytes, SUVMax and SUVMean pose no significant discriminative potential although their AUC's range from 0.602-0.625. AUC-values at $\mathrm{t}=0-15$ days and $\mathrm{t}=15-90$ days are 0.623 and 0.631 respectively, showing metabolic tumour volume is continuous in its discriminative potential, although it is not significant during the whole neoadjuvant treatment period. Subgroup-analyses show only the fraction of CEA-IM at $t=7-15$ days as significantly discriminative $(A \cup C=0.762, p=0.032$ ). In the overall treatment period $(\mathrm{t}=0-15$ days) and later time points this discriminative power is not reproduced. Significance may be erroneous due to $n=8$ but needs to be evaluated in a larger cohort to confirm. Other parameters in the subgroup-analysis show no significant discriminative power; only serum leucocytes $(n=21$ vs. $n=13)$ at $t=7-15$ shows an 
AUC of 0.659 and $p$-value of 0.184 , which is in line with AUC values at $t=0-7$ days and during $\mathrm{t}=0-15$ days as well.

Table 6.7 Calculated AUC-values of all parameters: AUC-values of the predicted probability for pCR as positive predicted outcome combined with the $95 \% \mathrm{Cl}$ and $\mathrm{p}$-values are shown in the table above.

\begin{tabular}{|c|c|c|c|c|c|c|c|c|c|}
\hline \multirow{2}{*}{\multicolumn{10}{|c|}{$\begin{array}{l}\text { ROC pCR (ypTONO) vs. non-pCR (>ypTONO) } \\
\text { (AUC pred prob) }\end{array}$}} \\
\hline & \multicolumn{9}{|c|}{ (AUC pred. prob.) } \\
\hline treatment moment/period & \multicolumn{3}{|c|}{$\mathrm{t}=0$ days } & \multicolumn{3}{|c|}{$\mathrm{t}=0-15$ days } & \multicolumn{3}{|c|}{$t=15-90$ days } \\
\hline parameters & AUC & $95 \% \mathrm{Cl}$ & $p$ & AUC & $95 \% \mathrm{Cl}$ & $p$ & AUC & $95 \% \mathrm{Cl}$ & $p$ \\
\hline serum CEA & $\underline{0,602}$ & $0.477-0.727$ & $\underline{0,141}$ & 0,393 & $0.261-0.526$ & 0,145 & 0,513 & $0.348-0.678$ & 0,887 \\
\hline serum CRP & $\underline{0,607}$ & $0.484-0.729$ & $\underline{0,123}$ & 0,438 & $0.309-0.567$ & 0,397 & 0,596 & $0.452-0.739$ & 0,306 \\
\hline serum Leucocytes & 0,557 & $0.428-0.686$ & 0,406 & $\underline{0,603}$ & $0.465-0.740$ & $\underline{0,169}$ & 0,587 & $0.421-0.752$ & 0,354 \\
\hline Flow \%parent Monocytes & 0,454 & $0.331-0.577$ & 0,502 & 0,522 & $0.389-0.655$ & 0,762 & 0,401 & $0.230-0.571$ & 0,289 \\
\hline Flow \%parent act. Mac. & 0,566 & $0.441-0.691$ & 0,338 & 0,447 & $0.307-0.586$ & 0,467 & 0,539 & $0.381-0.697$ & 0,676 \\
\hline Flow \%parent CEA-IM & 0,532 & $0.394-0.670$ & 0,646 & 0,556 & $0.406-0.707$ & 0,444 & 0,551 & $0.390-0.712$ & 0,585 \\
\hline SUVMax & $\underline{0,625}$ & $0.483-0.767$ & $\underline{0,089}$ & $\underline{0,628}$ & $0.473-0.783$ & $\underline{0,141}$ & 0,507 & $0.339-0.676$ & 0,938 \\
\hline SUVMean & $\underline{0,609}$ & $0.460-0.757$ & $\underline{0,140}$ & 0,564 & $0.400-0.728$ & 0,490 & 0,542 & $0.337-0.747$ & 0,752 \\
\hline MTV & 0,750 & $0.638-0.862$ & 0,001 & $\underline{0,623}$ & $0.477-0.768$ & $\underline{0,186}$ & $\underline{0,631}$ & $0.417-0.845$ & 0,320 \\
\hline \multicolumn{10}{|c|}{ ROC pCR (ypTONO) vs. non-pCR (>ypTONO) } \\
\hline (AUC pred. prob.) & \multicolumn{6}{|c|}{ subgroup-analysis } & & & \\
\hline treatment moment/period & \multicolumn{3}{|c|}{$t=0-7$ days } & \multicolumn{3}{|c|}{$t=7-15$ days } & & & \\
\hline parameters & AUC & $95 \% \mathrm{Cl}$ & $p$ & AUC & $95 \% \mathrm{Cl}$ & $p$ & & & \\
\hline serum CEA & $\underline{0,603}$ & $0.378-0.827$ & 0,389 & 0,414 & $0.211-0.617$ & 0,473 & & & \\
\hline serum CRP & 0,550 & $0.336-0.764$ & 0,674 & 0,432 & $0.243-0.622$ & 0,572 & \multirow{2}{*}{\multicolumn{3}{|c|}{$\begin{array}{l}\text { Table legend } \\
\text { value: AUC }>0.6\end{array}$}} \\
\hline serum Leucocytes & $\underline{0,633}$ & $0.428-0.837$ & 0,267 & $\underline{0,659}$ & $0.469-0.849$ & $\underline{0,184}$ & & & \\
\hline Flow \%parent Monocytes & 0,597 & $0.378-0.815$ & 0,425 & 0,582 & $0.352-0.811$ & 0,498 & \multicolumn{3}{|c|}{ value: $A U C>0.7$} \\
\hline Flow \%parent act. Mac. & $\underline{0,628}$ & $0.384-0.872$ & 0,291 & $\underline{0,620}$ & $0.346-0.893$ & 0,321 & \multicolumn{3}{|c|}{ sign. discriminating } \\
\hline Flow \%parent CEA-IM & $\underline{0,608}$ & $0.384-0.832$ & 0,373 & 0,762 & $0.588-0.936$ & 0,032 & \multicolumn{3}{|c|}{ Parameter if $\rightarrow$} \\
\hline SUVMax & na & na & na & na & na & na & \multicolumn{3}{|c|}{$95 \% \mathrm{Cl}[\geq 0.5-$ any $]$} \\
\hline SUVMean & na & na & na & na & na & na & \multicolumn{3}{|c|}{ (equals $p \leq 0.05$ ) } \\
\hline MTV & na & na & na & na & na & na & & & \\
\hline
\end{tabular}

\subsubsection{2 ypT0-2NO vs. ypT3-4NallMall}

At $t=0$ days serum CEA ( $p=0.096)$, serum CRP $(p=0.069)$ and MTV $(p=0.005)$ show AUC levels between 0.6 and 0.7. During neoadjuvant treatment period $t=0-15$ days no AUC levels above 0.6 are present. During $t=15-90$ days interval only serum leucocytes, the fraction of CEA-IM and MTV show an AUC above 0.6. Most discriminating parameters of the subgroup at $t=0-7$ days are the following: AUC values above 0.6 are shown for CEA, the fraction of activated macrophages and the fraction of CEA-IM. During $t=7-15$ days these are serum leucocytes $(A \cup C=0.758, p=0.018)$, and the fraction of monocytes $(A \cup C=0.600)$. 
Table 6.8 Calculated AUC-values of all parameters: AUC-values of the predicted probability for ypTO-2NO as the positive predicted outcome combined with the $95 \% \mathrm{Cl}$ and $\mathrm{p}$-values are shown in the table above.

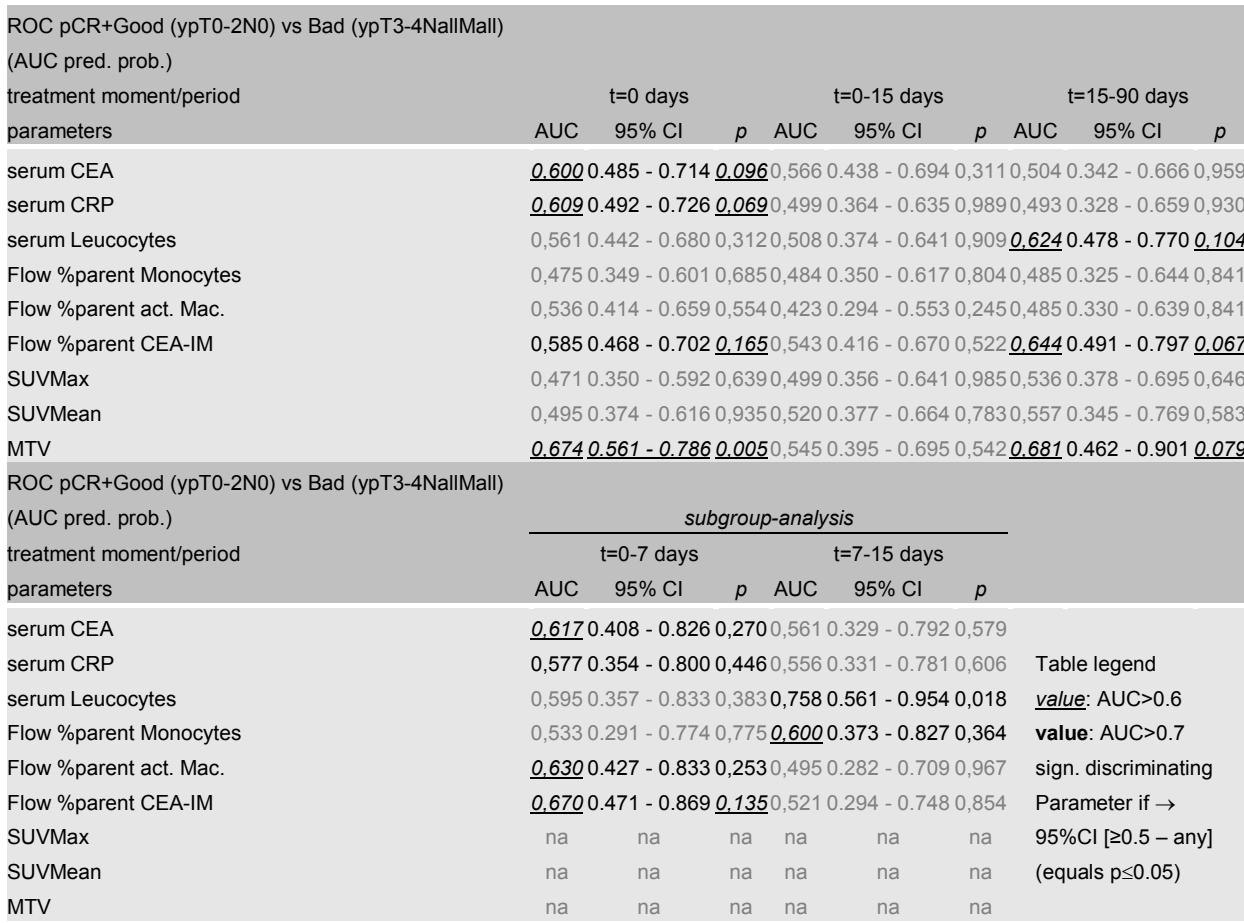

\subsubsection{Immune cell influx (INF) evaluation}

At $\mathrm{t}=0$ days only MTV shows an AUC level between 0.6 and 0.7 . At $t=0-15$ days MTV-values significantly discriminate $(A U C=0.777, p=0.002)$ between INF-High and INF-Low and during the waiting/recovery period $t=15-90$ days the MTV shows a trend to significance ( $A \cup C=0.722, p=0.078$ ). During the $t=0-15$ days interval CRP and SUVMax show AUC-levels above 0.6, only with CRP having a $p$-value of 0.183 . Between $t=15$ and $t=90 \mathrm{CRP}$, leucocytes, SUVMean show AUC-levels between 0.6 and 0.7 . During $t=0-7$ days $A U C$ values above 0.6 are shown for CEA, leucocytes, the fraction of monocytes $(p=0.168)$ and the fraction of CEA-IM. CRP seems significantly discriminative with an AUC of 0.821 $(p=0.019)$ in predicting INF-High outcome. Although patient numbers at $t=7$ $(n=7)$ could result in an erroneous calculation of AUC and $p$-value, AUC values on $t=7-15$ days, $t=0-15$ days and $t=15-90$ days are above 0.6 as well. During $t=7-15$ days the AUC values of CEA, CRP and leucocytes are above 0.6 but show no significance (Table 6.9). 
Table 6.9 Calculated AUC-values of all parameters: AUC-values of the predicted probability for INF-High as the positive predicted outcome combined with the $95 \% \mathrm{Cl}$ and $\mathrm{p}$-values are shown in the table above.

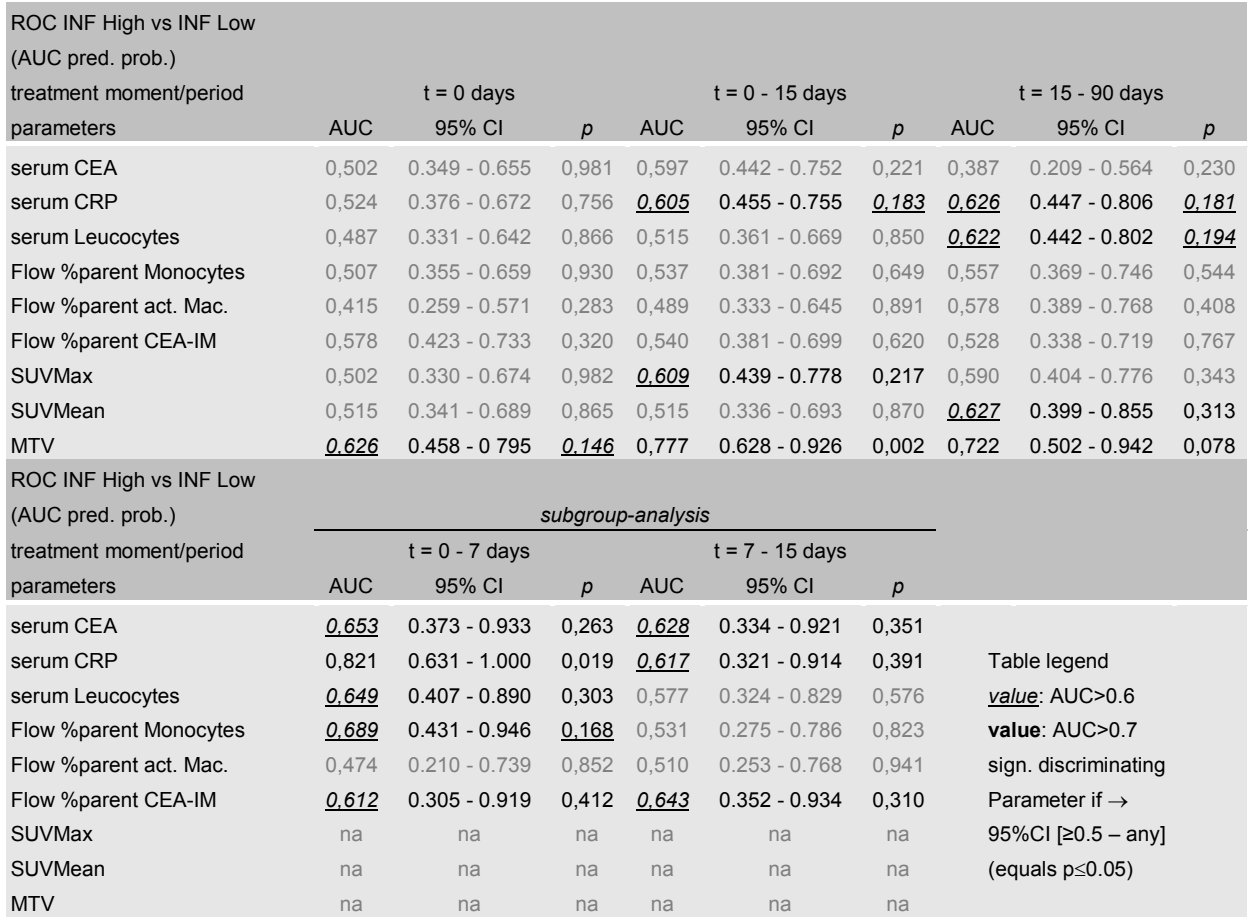

\subsection{Additional value to prediction models during nCRT}

Three prediction models, PET0, PET15 and PET90, were assessed for added value of the investigated biomarker parameters. All models predict $\mathrm{pCR}$ (ypTON0) vs non-pCR as outcome measure. The addition of all biomarkers of $\mathrm{t}=0$ to the PETO model increases the mean AUC-value from 0.73 to 0.75 . Adding $\triangle$ biomarkers $\mathrm{t}=0-15$ to the PET15 model showed a mean AUC-value of 0.94 $(95 \% \mathrm{Cl} 0.87-0.99)$. This is a significant increase in contrast to the AUC of 0.72 of the PET-CT-values alone at $t=15$ from previous studies ${ }^{16}$. The mean AUC of the PET90 model remained practically unchanged, showing an increase from 0.75 to 0.76 when adding all biomarkers to the PET-values. 


\section{Discussion}

This study evaluates parameters and parameter changes from different investigational modalities for their predictive capacity on outcome of nCRT in LARC patients. Several blood biomarkers and PET-CT parameters significantly discriminate in dichotomized outcome measures (such as TRG1-2 vs. higher, or ypTONO vs. higher) at multiple time points during nCRT. Adding the blood biomarkers to the existing prediction models strengthened their predictive value in predicting $\mathrm{PCR}$ during the first two weeks of $\mathrm{nCRT}$ prior to the waiting/recovery period. Thus, adding these markers to the PET15 model led to an increase of the AUC for the prediction of pCR to 0.94, well above the desired 0.90 . This could mean that changes between measured values during a treatment period could outperform the prediction capacity of stand-alone values measured at a fixed time point. The changes between time points seem to be better predictors than the values at a time-point themselves.

Such predictive information could play a role in deciding which definitive treatment strategy would follow nCRT: any form of surgical resection, or closewatching on the assumption of complete local disease control ${ }^{2,4}$. Thorough monitoring of such a patient every 3-6 months by clinical evaluation (such as digital rectal exam), blood analysis and imaging techniques (such as MRI, PET$\mathrm{CT}$ ) remain warranted, just as currently applied protocols describe. In the current study the added predictive value of blood borne biomarkers for two different outcome measures were analysed: TRG, ypTN. The local biological effect of $\mathrm{nCRT}$ was investigated by immune cell influx evaluation.

\subsection{TRG outcome measure}

Together with MTV, serum CEA is an accurate discriminator for TRG 1-2 at $t=0$. The $t=0$ finding of CEA is concurrent with earlier findings by Buijsen et al. ${ }^{9}$, when predicting ypTN (pCR and ypT0-2N0) as an outcome measure. The similar results for ypTN and TRG in two different studies support the view that CEA at $\mathrm{t}=0$ is a significant discriminator for tumour response and subsequent tumour regression.

During the waiting/recovery period starting at day 15 , continuing until day 90 after the start of $\mathrm{nCRT}$ changes of serum CEA, the fraction of monocytes, SUVMax and SUVmean display predictive capacity. Their addition to established outcome prediction models increases pre-existent AUC-values. This may be clinically relevant as inclusion of (a combination of) these parameters could 
change the choice for the type of surgery performed, e.g. an abdominal perineal resection (APR), low-anterior resection (LAR) or a TEM. Restaging by PET-CT prior to surgery revealing new SUVMax and SUVMean values correctly discriminates between TRG 1-2 stages and TRG3 and higher stages. However, performing a PET-CT evaluation at that point in time is beyond the decision moment on type and duration of treatment strategies. In contrast, monitoring at even smaller time intervals, and closer to the start of the nCRT yielded results potentially capable leading to adjustment of the nCRT strategy. Thus, data derived from the subgroup of patients that underwent additional sampling at day 7 post start of treatment suggest that changes of CRP and the fraction of monocytes over the period 0-7 days and the period 7-15 days are significantly predictive for TRG 1-2. As this observation was made in a small albeit randomly selected subset of patients it needs confirmation.

\section{2 pCR vs. non-pCR as outcome measure}

Determination of MTV with PET-CT at $\mathrm{t}=0$ predicts $\mathrm{pCR}$ accurately in this study population. Repeated MTVs in subsequent measurements up until 90 days retain this predictive power, with AUC's ranging from 0.61 to 0.76 . Monitoring MTV during the first two weeks could provide insight in the chance to reach $\mathrm{pCR}$, although MTV did not show significant discriminative power in subsequent measurements. Subgroup analyses showed delta fraction of CEA-IM was significantly discriminative during the second treatment week. The levels of leucocytes show AUC's above 0.6 during the first and the second neoadjuvant treatment week and over the total initial two week period ( $\mathrm{t}=0-15$ days). Thus, determination of leucocyte numbers seems to be predictive for $\mathrm{pCR}$ during the first two weeks of nCRT. Evaluation of additional value to a treatment period prediction model is warranted. Such a model needs to be developed.

The increase of the fraction of activated macrophages in the non-pCR group (graph 2E) almost appears to be delayed compared to its counterpart in the pCR group. The reaction-level in the non-pCR group is slightly lower and appears postponed to $t=15$. To interpret these results one could postulate that the pCR group is able to react to the combination of tumour, chemotherapy and radiotherapy earlier than the non-pCR group leading to a complete response within the timeframe of around 90 days. This difference in reaction time is present in ypT0-2N0 vs ypT3-4NallMall (graph 3E) as well, although bad response groups show a higher fraction of activated macrophage values at the later $\mathrm{t}=15$ time point. Evaluating the fraction of activated macrophages in TRG 
$1-2$ vs $\geqq$ TRG 3 (graph $1 \mathrm{E}$ ) shows practically the same pattern as ypT0-2N0 vs. ypT3-4NallMall. Interpreting these results as a postponed or inadequate immune reaction in the non-pCR ( $\geqq T R G \quad 3$ and ypT3-4) outcome groups seems plausible.

\subsubsection{Good responders vs. Bad responders outcome}

As MTV remained a significant predictor for good response at $t=0$ (as in the $p C R$ vs non-pCR bivariate analysis) it can inform clinicians early about a patient chances when nCRT is initiated. When a predictive value indicates a high chance for bad response, treatment could be intensified or performing surgery earlier could become an option to consider. CEA and CRP both show an AUC of above 0.6 , with a trend to significance for CRP, indicating that both should be included in multi-parameter prediction models. Increasing patient numbers may further push these trends towards a pCR or non-pCR outcome.

\subsection{Immune cell influx evaluation by INF-score}

In $62 \%$ of our investigated patients an INF-score could be interpreted from standard pathology reports. This information-yield could easily be improved to $85-90 \%$ or higher if further standardisation of reporting would be adopted. The simplicity of the score is one of its advantageous characteristics, leading to quick adoption.

Assessing the number of monocytes, activated macrophages and CEAcontaining macrophages (CEA-IM) during nCRT of rectal cancer patients was hypothesised to be an effect-measurement of the biological ability of proper cell influx for anti-tumoural immune action around or inside the tumour. Lower blood levels would indicate increased influx. However, the results do not support this hypothesis.

MTV shows the ability to discriminate between INF High and INF Low on all measured time points with an AUC above 0.6, even with significance during the period $\mathrm{t}=0-15$ days and $\mathrm{t}=15-90$ days. Monitoring MTV makes it possible to predict the influx of immune cells and fibrosis to a certain level already in the first two weeks of neoadjuvant treatment. This result may not only provide treatment alteration possibilities but more importantly, it may enable monitoring strategies. Baseline MTV-value is already predictive for the final INF-score, hence no neoadjuvant treatment was initiated at $t=0$. This could mean that a patients own immune system reaction to tumour presence or disease-load is reflected in the MTV-value. The highest (significant) AUC-value was calculated between $\mathrm{t}=0$ and $t=7$ for serum CRP in the subgroup-analysis of the INF-score evaluation, 
indicating CRP-level changes during early treatment may be predictive for immune cell influx. Further investigation, and subsequent validation of the INFscore in larger patient cohorts is needed because of these interesting observations.

\subsection{Clinical value of (flow cytometrical) blood biomarkers}

Combining PET-values with the 6 measured blood biomarkers increased the pCR-prediction power of the PET15-model significantly to an AUC of 0.94. This means that when using the updated prediction model, one can predict ypTON0outcome $(\mathrm{pCR})$ with $94 \%$ certainty based on available clinical data, blood biomarkers and PET-CT-values focusing on the neoadjuvant treatment period $t=0-15$ days. This is a important improvement of previously generated prediction models. Patient numbers decrease after imputation ( $n$ decreases from 82 to 58 for PET-15 model, for PET-90 model it decreases from 59 to 41). This means applying this model in the clinical setting would be premature and needs further investigation with larger cohorts, without the need for imputation techniques. Based on TRG results, validation of the following parameter-panel needs to be performed in a second study to predict.

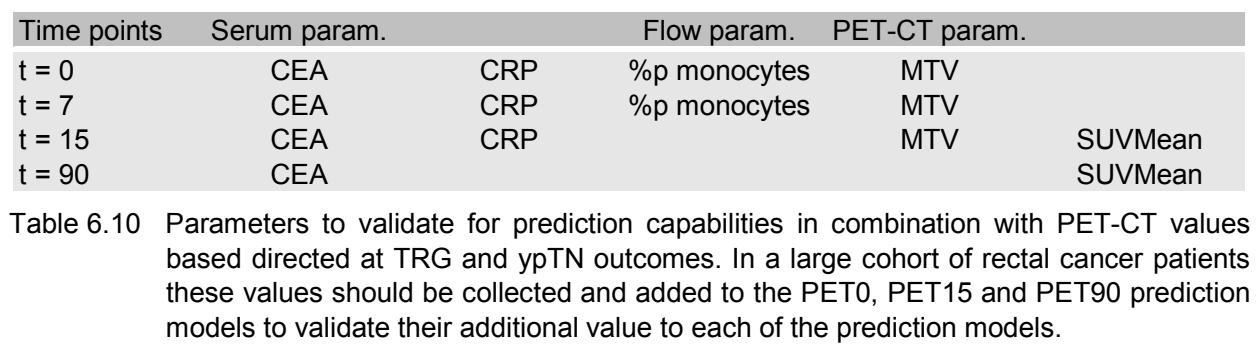

\subsubsection{Translation to clinical setting}

Translation to the clinical setting would be a secondary goal. Thus, changing $\mathrm{nCRT}$ or surgical treatment choices could become based on reliable prediction outcomes once they have been validated. If treatment strategy adjustment could be accomplished without the need for imaging techniques patients would be relieved of additional radiation burden. When applying shared decision making, physicians and patients could profit from this enhanced prediction model in this process. An online prediction calculator could simplify applying the new model during outpatient consults. Applying the monitoring ability during the first 2 weeks of treatment, a radiotherapist could at least predict with a $94 \%$ certainty if 
the patient is a potential candidate for a wait-and-see policy or will need a resection. Whether the prediction-models could eventually lead to enhancing the nCRT-treatment or, in case of no remaining nCRT options, stopping nCRT and performing early surgery needs to be investigated.

\subsection{Conclusion}

pCR-response prediction during the first two weeks of chemoradiotherapy based on a combination of PET-CT-values and blood biomarkers is feasible as illustrated in the TRG and ypTN outcome measures. CEA and MTV at $\mathrm{t}=0$ are significantly discriminative in TRG outcome prediction in patients during neoadjuvant treatment. In the ypTN outcome prediction only MTV reaches significance. Although TRG and ypTN show similar trends in pCR prediction, TRG is considered the gold standard and should therefore be used to validate response prediction models before clinical application. To gain insight in the biological effects of the nCRT during early neoadjuvant treatment, Further investigation on blood biomarker values is warranted. 


\section{References}

1. Zhang C, Tong J, Sun X, Liu J, Wang Y, Huang G. 18F-FDG-PET evaluation of treatment response to neo-adjuvant therapy in patients with locally advanced rectal cancer: A metaanalysis. Int J Cancer 2012;131:2604-11.

2. Beets GL. Critical appraisal of the 'wait and see' approach in rectal cancer for clinical complete responders after chemoradiation (br J surg 2012; 99: 897-909). Br J Surg 2012;99:910.

3. Glynne-Jones R, Hughes R. Critical appraisal of the 'wait and see' approach in rectal cancer for clinical complete responders after chemoradiation. Br J Surg 2012;99:897-909.

4. Maas M, Beets-Tan RG, Lambregts DM, Lammering G, Nelemans PJ, Engelen SM, et al. Waitand-see policy for clinical complete responders after chemoradiation for rectal cancer. J Clin Oncol 2011;29:4633-40.

5. Lee NK, Kim DY, Kim SY, Oh JH, Park W, Choi DH, et al. Clinical outcomes of local excision following preoperative chemoradiotherapy for locally advanced rectal cancer. Cancer Res Treat 2014;46:158-64.

6. Bökkerink GM, de Graaf EJ, Punt CJ, Nagtegaal ID, Rütten H, Nuyttens JJ, et al. The CARTS study: Chemoradiation therapy for rectal cancer in the distal rectum followed by organ-sparing transanal endoscopic microsurgery. BMC Surg 2011;11:34.

7. Rosenberg R, Herrmann K, Gertler R, Künzli B, Essler M, Lordick F, et al. The predictive value of metabolic response to preoperative radiochemotherapy in locally advanced rectal cancer measured by PET/CT. Int J Colorectal Dis 2009;24:191-200.

8. Denecke T, Rau B, Hoffmann KT, Hildebrandt B, Ruf J, Gutberlet M, et al. Comparison of CT, MRI and FDG-PET in response prediction of patients with locally advanced rectal cancer after multimodal preoperative therapy: Is there a benefit in using functional imaging? Eur Radiol 2005;15:1658-66.

9. Colotta F, Allavena P, Sica A, Garlanda C, Mantovani A. Cancer-related inflammation, the seventh hallmark of cancer: Links to genetic instability. Carcinogenesis 2009;30:1073-81.

10. Molla M, Panes J. Radiation-induced intestinal inflammation. World J Gastroenterol 2007;13: 3043-6.

11. Buijsen J, van Stiphout RG, Menheere PP, Lammering G, Lambin P. Blood biomarkers are helpful in the prediction of response to chemoradiation in rectal cancer: A prospective, hypothesis driven study on patients with locally advanced rectal cancer. Radiother Oncol 2014;111:237-42.

12. Mandard AM, Dalibard F, Mandard JC, Marnay J, Henry-Amar M, Petiot JF, et al. Pathologic assessment of tumor regression after preoperative chemoradiotherapy of esophageal carcinoma. Clinicopathologic correlations. Cancer 1994;73:2680-6.

13. Japink D, Leers MPG, Sosef MN, Nap M. CEA in activated macrophages. New diagnostic possibilities for tumor markers in early colorectal cancer. Anticancer Res 2009;29:3245.

14. Japink D, Nap M, Sosef MN, Nelemans PJ, Coy JF, Beets G, et al. Reproducibility studies for experimental epitope detection in macrophages (EDIM). J Immunol Methods 2014;407:40 - 7.

15. Janssen MH, Ollers MC, Riedl RG, van den Bogaard J, Buijsen J, van Stiphout RG, et al. Accurate prediction of pathological rectal tumor response after two weeks of preoperative radiochemotherapy using (18)f-fluorodeoxyglucose-positron emission tomography-computed tomography imaging. Int J Radiat Oncol Biol Phys 2010;77:392-9.

16. van Stiphout RG, Lammering G, Buijsen J, Janssen MH, Gambacorta MA, Slagmolen P, et al. Development and external validation of a predictive model for pathological complete response of rectal cancer patients including sequential PET-CT imaging. Radiother Oncol 2011;98: 126-33.

17. van Stiphout RG, Valentini V, Buijsen J, Lammering G, Meldolesi E, van Soest J, et al. Nomogram predicting response after chemoradiotherapy in rectal cancer using sequential PETCT imaging: A multicentric prospective study with external validation. Radiotherapy and Oncology 2014;113:215-22. 
Predicting response using blood biomarkers and PET-CT during neoadjuvant chemoradiation

18. DeLong ER, DeLong DM, Clarke-Pearson DL. Comparing the areas under two or more correlated receiver operating characteristic curves: A nonparametric approach. Biometrics 1988;44:837-45. 



\section{CHAPTER 7}

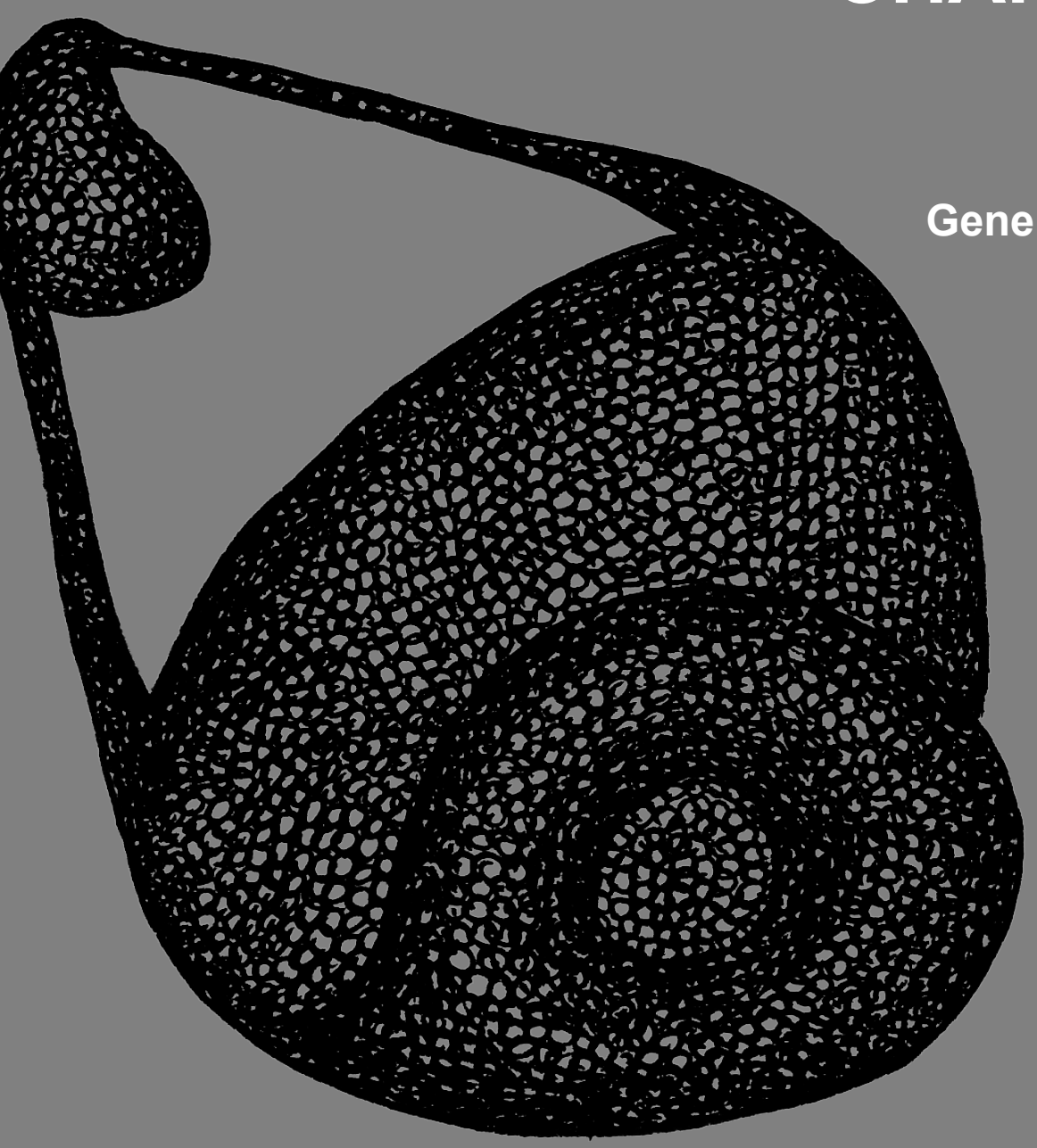


th 


\section{General discussion}

This thesis reports on the development, cellular background, feasibility and early application of an experimental diagnostic assay detecting epitopes on tumour related material in the cytoplasm of monocytes/macrophages circulating in peripheral blood (epitope detection in macrophages (EDIM)). Although biological variation was found as the key interference factor, early application was shown to have additional value in monitoring neoadjuvant treatment in rectal cancer patients. This enables clinicians to initiate treatment evaluation during the early stages of neoadjuvant treatment and to alter treatment if needed. Refinement of the assay may lead to further additional value. The investigations in this project focussed on CRC as a research model. The principles could be valid in other tumours as well, because of their general applicability to immune cell research.

\section{Cellular background}

Monocytes are known as the precursors of macrophages. Differentiation from one into the other can occur after intravascular activation by rolling and crawling over the intraluminal vascular wall, connecting to various receptors and subsequent extravasation ${ }^{1}$. Recruitment into tissue is initiated by chemotaxis, local cell-signalling, hypoxia and by gradients of all three ${ }^{2}$. Monocyte heterogeneity research shows three distinct subsets CD14++CD16- (classical), CD14+CD16++ (non-classical) and CD14++CD16+ (intermediate, including atypical macrophages and dendritic cells $)^{3-5}$. Intermediate monocytes have been described to be more prevalent in primary CRC than in metastatic disease ${ }^{6}$. They could therefore be the most important population to investigate within the monocyte-macrophage system for more localised disease detection in CRC. The monocyte-macrophage system is highly versatile, it reacts in both a tissue and/or organ specific manner, as well as a situation specific manner. Monocytes and macrophages in breast (cancer) tissue ${ }^{7-10}$ have been shown to act differently than those in the intestinal tract to the presence of malignancy ${ }^{11,12}$. The difference between monocytes and macrophages could be less strict than described before ${ }^{13}$. Recent literature describes monocytes and macrophages more and more as parallel existing cell-types besides the known roles of predecessor and successor. Monocytes are known to extravasate, ingest pathogens, migrate to lymphoid organs via lymphatic vessels and differentiate into antigen presenting cells (dendritic cells or macrophages). Secondly they can migrate in the lymph nodes as monocytes/macrophages. Finally, monocytes can 
be recruited directly from the blood into an inflamed lymph node via high endothelial venules ${ }^{14}$. Macrophage functions do not differ much from monocytes in the beginning. Macrophages are also known for their migratory and phagocytic capacities. The ability of macrophages to intravasate after substance uptake has been shown in murine models ${ }^{15}$. This phenomenon resembles the migratory ability of macrophage-tumour-cell hybrids. It could therefore be suggested that a continuum of myeloid derived (precursor) cells exists, almost a more general phagocyte, with a gradual change in surface markers during maturation ${ }^{6}$. These cells are able to act in an undifferentiated form ${ }^{16}$, differentiate into more specialised cell types with antigen-presenting or digestive functions after pathogen uptake ${ }^{14}$ and are even able to fuse with other (parts of) cells ${ }^{17-20}$. Based on both this continuum and the ability to return to the bloodstream, our studies hypothesised a blood-tissue circle in which monocytes and macrophages play a leading role in health, (chronic) inflammatory or malignant disease, such as CRC. The possible role monocytes and macrophages could play in (colorectal) cancer diagnostics have been described in chapter 1.

\subsection{The third monocyte subtype}

In our CRC-studies we focussed on intermediate monocytes (CD14++CD16+). At the start of our studies this third subtype, intermediate monocytes were still quit unknown and not generally used as a separate entity in monocyte heterogeneity studies. Therefore we always referred to the investigated population as CD14+CD16+ macrophages. In literature, the CD14++CD16+ subtype has recently been described as being positive for a range of markers, including CD64, CD115, CD163, CCR2 and CX3CR1. Also MHC class II was specifically high in intermediate monocytes ${ }^{21}$. Furthermore, we did not investigate other common monocyte and macrophage markers, such as CD11a, CDC11b, CD68 or HLA-DR, for their presence on the cells in our samples ${ }^{22}$ to stay as close as possible to the initial selected CD-marker palette. On the other hand we did perform flow cytometrical sorting which showed the CD14+CD16+ peripheral blood monocytes as irregular cells with large lobular cores, mimicking typical macrophage morphology. This finding has recently been confirmed by Mandl et al, characterising thawed human CD14++CD16+ bone marrow cells ${ }^{22}$. They found the CD14++CD16+ subset to be primarily enriched in human bone marrow and in lowest concentration in peripheral blood. Thus, other accessible sampling locations other than peripheral blood could provide a higher yield, considering these findings. In future studies these locations should be explored. 
Further differentiation research could have brought us closer to answering the question of the monocyte-macrophage difference in this CRC model. On the other hand, it would have detached the performed research further from earlier promising results from our group in prostate cancer. Our primary goal was to reproduce those results in $\mathrm{CRC}$, keeping most parameters equal to the prostate studies.

\subsection{Monocyte heterogeneity in murine and human research}

Many studies of monocyte heterogeneity are based on murine models. Mouse to human translation indeed has the advantage providing insight in functioning of the immune system without depending on human experiments. To confirm macrophage subtypes and functions found in mice, and which are present in human tumours, inter-species monocyte-subset and monocyte-marker mapping is needed. This mapping has been performed, but not all subsets have been shown to be equal ${ }^{3,23,24}$. For tumour-associated macrophages this mapping has been performed as well. Comparable macrophage populations exist, given that the individual TAM expression signatures derived from mice are represented in human tumour datasets and can even be predictive ${ }^{25}$. Newly developed fluorescence based mouse experiments show promising results, although the claimed results remain based on genetic murine models ${ }^{26}$. Animal experiments investigating tumour transplantation in immunocompetent animals should be interpreted cautiously. Reported outcomes are weakened by confounding factors, such as antigraft reactions (even in syngeneic contexts), as well as the skewed immune reactions in immunocompromised mice ${ }^{27}$. Therefore, research utilising human materials and complementary biomarkers should always be the preferred strategy, whenever feasible.

\subsection{Intracellular macrophage tumour marker presence}

In human lymphoid, CRC-tumour and CRC-tumour surrounding tissue the presence of CD14+CD16+ macrophages staining intracellularly positive for CEA,

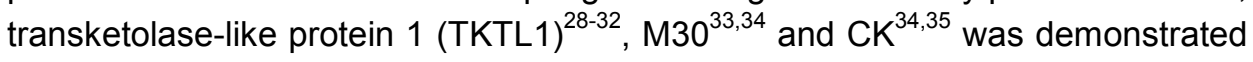
using light microscopy (LM) (chapter 2). The reproducibility of the epitope detection in macrophages method (EDIM) has been assessed by investigating the latter four markers in the validation study (chapter 5). This chapter shows results about reproducibility of the EDIM-assay using multiple markers. These results illustrate monocyte/macrophage uptake versatility. Whichever non-self or degraded entity is encountered, it is ingested and digested and possibly 
subsequently presented. Using electron microscopy (EM) we attempted to answer the question of where in the macrophage the tumour marker-specific epitope, connected to the tumour related material, would be situated. In some of the morphologically identified macrophages, tumour markers appeared to be on internalised villous cell-parts inside vesicles situated in the cytoplasm. These vesicles most likely represent lysosomes. Even though CEA was still detectable in some vesicles, not all vesicles showed tumour marker positivity, which is concurrent with the LM and flow cytometry results. This part of the project made clear that CEA was not actively presented on the cell-surface or excreted from the macrophages, but rather internalized and probably subsequently digested. This digestion process did not prohibit intracellular CEA detection. Furthermore, CEA was easily detected in the villous linings of CRC in almost all specimens (chapter 3). The study results show that morphologically identified macrophages were able to internalise tumour related material. It was not possible to immunochemically prove that the macrophages were positive for CD14 and/or CD16 in chemically fixed, resin embedded tissue samples prepared for microscopy (light- and electron microscope). Therefore there is the possibility that the identified cells were in fact another type of phagocyte, although morphology resembles that of macrophages. New attempts to successfully apply a double staining strategy for monocyte/macrophage identification and tumour marker presence should be pursued.

\section{Development and feasibility of the technique}

\subsection{Development of the peripheral blood macrophage assay}

Translation of the peripheral blood prostate cancer macrophage assay ${ }^{36-40}$ to CRC proved to be feasible using established tumour markers (chapter 4). CEA was investigated as the primary tumour marker, utilising earlier research by our group to select the most specific antibody directed against CEA ${ }^{41}$. Performing the assay with the buffy coat filtrate layer of EDTA whole blood using a CPTfiltration tube (Becton Dickinson, California) sometimes introduced a monocyte yield problem. This yield appeared to be inconsistent between CPT-tubes. Increasing the total number of events counted by flow cytometer by raising the stopping gate event-number solved this. This solution subsequently introduced a low cell count problem in thin buffy coat layers in a minor part of the included patients. For the duration of the investigation this was accepted, enabling continuity of the same SOP during all experiments. However, some patients had 
to be excluded due to low cell counts. This technical issue may have induced a selection bias, probably only affecting low-cell samples. Were patient numbers affected with low cell counts due to higher cell turnover for host-defence reaction regulation? Or did it affect patient numbers that have insufficient immune cell counts due to disease progression? Currently both questions have remained unanswered. In future research the low yield could be addressed by replacing the use of the filtration tube in the SOP by performing a red blood cell lysis in the whole blood sample ${ }^{42}$. Increasing the monocyte yield has been described in other studies investigating the CD14/CD16-monocyte population ${ }^{31,43}$. Yield improvement was attained successfully without the disturbance of red blood cell clotting, utilising the red blood cell lysis technique.

\subsection{Pilot study}

By combining the experimental assay with routine serum c-reactive protein (CRP) measurements healthy individuals could be distinguished from patients with chronic inflammatory or malignant bowel diseases (chapter 4). Based on AJCC staging for CRC it was possible to distinguish between AJCC I to AJCC IV stages. In addition to CEA, also M30 and CK were also detectable in varying numbers of macrophages in each patient. In contrast to CEA, M30 and CK were not able to significantly distinguish AJCC stage I to IV groups (unpublished data). The consecutively included CRC patients were diagnosed at our outpatient clinic without any form of prior selection. The co-morbidity level of patients in our region is known to be higher than on average in the Netherlands, and may prove to be higher in relation to other western countries as well. Therefore a control study in another region or country should be performed before new clinical studies (in either follow-up or screening setting) should be started. In diagnostic tool development this is considered part of standardised protocol. In the pilot study, healthy hospital personnel were included from a similar age range. The included number of benign disease cases was relatively low in this pilot study. This may have led to overestimation of the difference in the fraction of monocytes and CEA-IM between benign and malignant disease cases. In a new control study a larger cohort of benign disease cases should be investigated to rule out possible influences induced by the small number of benign disease patients. 


\subsection{Validation of diagnostic test}

In diagnostic test evaluation, not only patient controls need to be investigated, but biomarker control application as well. In the pilot study investigating the EDIM-method, isotype controls were applied to correct for background signals. Sometimes strange cell population shifts occurred during the pilot inclusion period, leading to exclusion of certain patients because of outcome interpretation issues. Investigating a possible solution for the isotype control problems led to the introduction of a modified fluorescence-minus-one (mFMO) control. Applying this type of control resolved the issue of the shifting cell populations, but it changed the pilot-SOP. During a transition period, double controls (isotype alongside $\mathrm{mFMO}$ ) were used in the studies. No explanation was found for the cell population shifts. For that reason the mFMO was permanently introduced as the primary control. According to literature an FMO is the preferred control in these types of multi parameter flow cytometrical tests ${ }^{44}$. However, the difference with the pilot study remains. The mFMO creates the possibility for undesired interpretation differences, which thus remain undetected after permanent mFMO application. During the validation period the gating strategy was slightly altered as well, which was recently proven to actually be more competent to investigate the earlier mentioned intermediate monocytes ${ }^{21,45}$. The focus on this subtype alone was not all that was changed. Considering larger and more complex CD14++CD16+ cells as the cell-type of interest, height and width of the forward scatter (FSC)-signal were investigated (Figure 7.1A-C) along the CD14 and CD16 positivity to separate debris from intact cells. The characteristic maturation/activation curving of the "CD14CD16-population-continuum" showed the larger and more irregular cells to be just on the outside of the corner of the curve (Figure 7.1B), mostly at the maximum of CD14 and CD16 positivity (unpublished results). These cells being doublet cells or aggregates would not have positioned them exactly on the edge of the maturation curve. In CRC patients the population is interesting, as it was described in literature that the intermediate monocyte subtype is considered a more sensitive diagnostic indicator of CRC compared to other subtypes ${ }^{6}$.

By performing different relevant reproducibility tests multiple times the macrophage assay has been proven technically reproducible. Biological variation has been found as the main interference factor (chapter 5) in healthy control subjects. The (activated) monocyte population is varying over time, which could be a reason to establish a normal range for each patient. If fluctuation 
remains within this range, no further action is needed. The problem with this approach is that a normal range would have to be established in healthy conditions for each individual. That would account for the population as a whole. In current tumour marker research and quality control studies another approach is applied. A change of more than $25 \%$ of a test result of an established serum tumour marker occurring in two subsequent measurements is considered clinically relevant $(25 \% \text { twice in a row approach })^{46-48}$. Further follow-up of the patients included in our research population need to confirm the applicability of this rule to the new assay. The biological variability issue could be solved utilising this approach. The follow-up period of the investigated population is currently over two years postoperatively, enabling the analysis of these results in relation to the mentioned "25\% twice in a row" approach.

\section{Early clinical application in rectal cancer patients}

During nCRT a substantial cell shedding effect is hypothesised, not only in the rectal tumour, but in the patient as a whole. All body cells receive the same chemotherapy dosage and locally the tumour area receives frequent radiotherapy. Ingestion by macrophages of shedded (parts of) tumour cells and tumour related material should result in detectability by the EDIM-test. Blood levels of shedded material are hypothesised to be high in this setting, and may be distinguished more easily from a continuous background signal and persistent biological variability. Therefore, the population of rectal cancer patients during nCRT was chosen for early clinical application of the EDIM-test.

\subsection{Choice of patient population}

Loco-regional control is the main goal of pre-surgical radiochemotherapy in rectal cancer patients. The benefit of decreasing tumour size and destruction of metastasis in regional lymph nodes enables limited resections ${ }^{49}$ and reduces (metastatic) recurrence respectively. The pre-treatment period of rectal cancer patients is intriguing because of malignant cell development combined with counter-events induced by radiochemotherapy. Inflammation, DNA-damage and cell-decay ${ }^{50}$ are only some of the effects of the combination of rectal cancer and neoadjuvant treatment ${ }^{51,52}$. Not every patient benefits from neoadjuvant treatment. Recent publications report that approximately $50 \%$ of the patients respond to neoadjuvant chemoradiotherapy. Of that group only $20-25 \%$ shows 
complete pathological response ${ }^{53}$. The last category warrants a wait-and-see policy $^{54-56}$. The non-responders should become candidates for (experimental) alternative neoadjuvant treatment strategies. If no other options remain, early surgery could be an option; otherwise palliative treatment should be offered. Distinguishing patients before and during radiochemotherapy, based on pretreatment values and response measurements during early treatment, was the goal of our research (chapter 6).
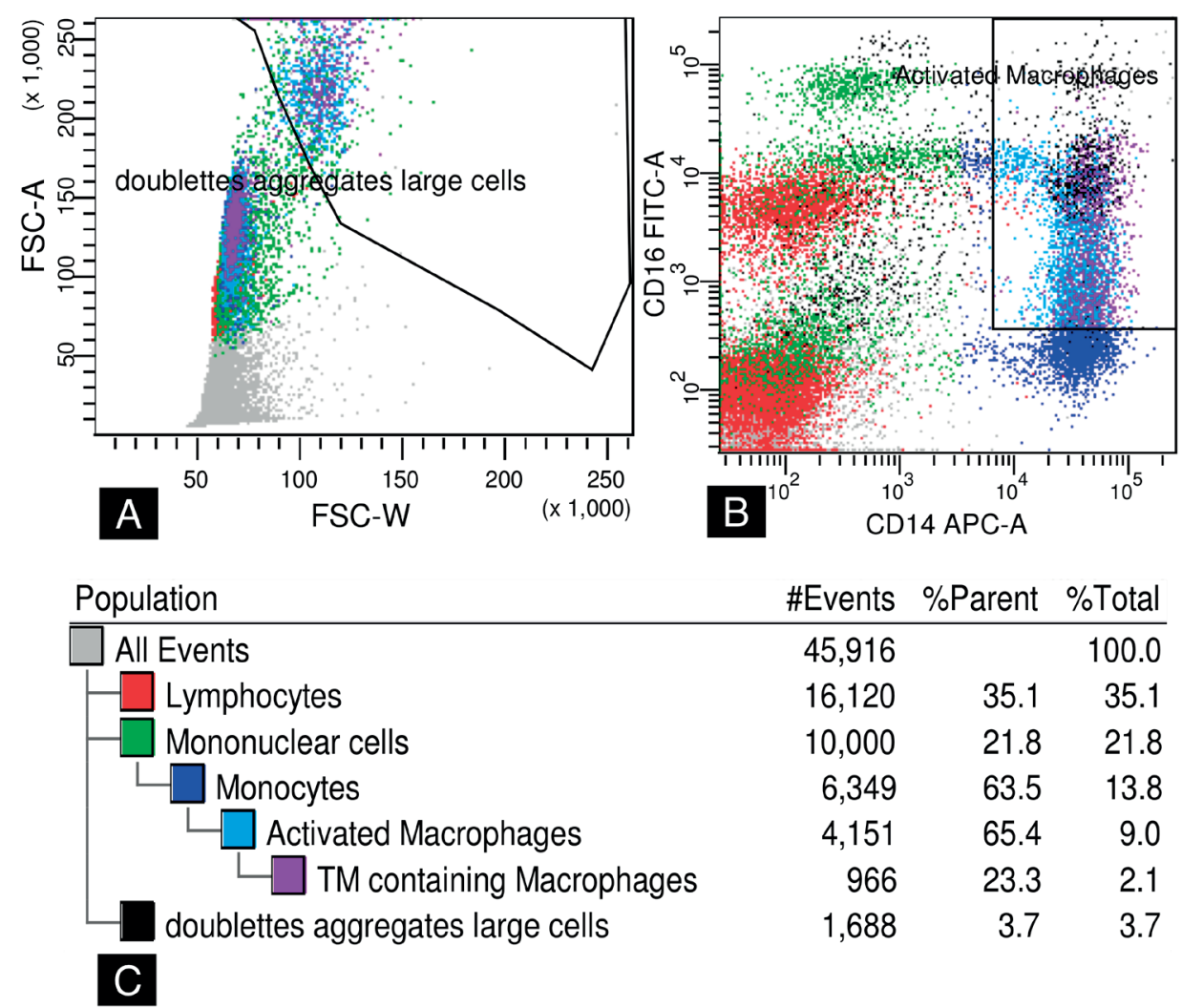

\begin{tabular}{rrr} 
\#Events & \%Parent & \%Total \\
\hline 45,916 & & 100.0 \\
16,120 & 35.1 & 35.1 \\
10,000 & 21.8 & 21.8 \\
6,349 & 63.5 & 13.8 \\
4,151 & 65.4 & 9.0 \\
966 & 23.3 & 2.1 \\
1,688 & 3.7 & 3.7
\end{tabular}

Figure 7.1 Identification of large cells applying a back-gating strategy using forward scatter height and width properties $(A)$. In the hierarchy $(C)$ is displayed that this intermediate monocyte population comes forth from all events and is mostly displayed on the corner of the CD14++CD16+ activation/maturation curve $(B)$ inside the activated macrophages selection area (unpublished results). In the performed CEA-IM experiments this localisation was not always as explicit as in this example. 
Predicting a patient's benefit to radiochemotherapy should be the main investigative outcome measure in this area of research. Investigating the possibility to monitor the response and maybe even evaluating the methods of the response assessment could be another goal of this type of research. Established surveillance protocols are considered safe and proposing changes based on blood biomarker outcomes may not be accepted directly. This should not stop innovation in the research-setting; the rectal cancer wait-and-see policy for complete responders after neoadjuvant chemoradiotherapy was also considered controversial at first ${ }^{54,56-60}$.

\subsection{Response prediction}

Response prediction to neoadjuvant chemoradiotherapy is not generally practiced $^{61,62}$. Only a select few groups are showing experimental results. These results suggest mainstream application is possible ${ }^{63-65}$, although they are not always concurrent ${ }^{66}$. Measurement of response without adding an extra risk factor (or patient burden), such as additional radiation, would be preferable. PET-CT-scans deliver large amounts of additional radiation; MRI on the other hand does not ${ }^{57}$. Both entities have shown monitoring capabilities, with the best results currently for FDG-PET-CT ${ }^{67,68}$. Major drawbacks of these techniques are that they are expensive, in addition to patient discomfort during these scans that take up to 45 minutes. The use of blood biomarker assay(s) could fill in a gap. The challenge is to find a (panel of) biomarker(s) that differentiates enough to predict response before or in early radiochemotherapy phases. Up until now, no biomarker or biomarker panel has proven to have enough predictive power by itself $^{69,70}$ Additional imaging modalities are able to boost the predictive capacity (AUC's) to acceptable levels ${ }^{71}$. The occurrence of response is defined pathologically as decrease in tumour size, no (vital) remaining tumour cells (or tumour cell budding ${ }^{72}$ ) and no lymph nodes with vital tumour tissue. Recently added were local fibrosis and influx of immune cells $\mathrm{s}^{70,73,74}$. Combining PET-CT with serum (tumour) markers (75) as well as inflammatory parameters was hypothesised to be able to reflect the local and systemic reactions of the patient on radiochemotherapy. Chapter 6 reports on the results of a nine-parameter analysis, combining serum biomarkers with the experimental monocyte/ macrophage assay and FDG PET-CT results. Focussing on TRG and ypTN response assessment, it was shown that serum CEA and metabolic tumour volume can be used as predictors at the start of radiochemotherapy. Adding a 
monocyte-fraction analysis boosted the predictive capacity of the prediction models during the first two weeks of radiochemotherapy.

\subsubsection{Immune system applicability in response prediction}

Whether the immune system could be addressed as a possible predictive factor is debatable. Bone marrow depression is a known side effect in patients receiving radiochemotherapy ${ }^{51}$. This effect was also reflected in lower monocytelevels in rectal cancer patients in our studies (unpublished data), although the activated monocyte-population within the whole monocyte population did increase during the radiochemotherapy. The mentioned biomarkers were not predictive enough by themselves, and still needed the combined analysis with FDG PET-CT. However, the predictive value of earlier prediction models was increased substantially though (chapter 6). Further biomarker research may enable a blood biomarker-panel composition sufficiently predictive to indicate whether a scan is needed to objectify the response or even to refrain from scanning. Once validation has been performed in multiple patient groups, the application of a biomarker panel could lead to a decrease of the additional radiation burden for neoadjuvant treated rectal cancer patients.

\section{Future developments, challenges and recommendations}

\subsection{The monocyte or macrophage continuum}

Investigating other common monocyte and macrophage markers, such as CD11a, CD11b, CD64, CD68, CX3CR1, TLR-2, TLR-4 or HLA-DR, should be used to elaborate on the roles of specific mononuclear cell and monocyte subtypes in future investigations ${ }^{76,77}$. Identifying these in relation to macrophage marker presence could inform about marker overlap in certain situations, enabling monocyte, macrophage or subtype identification. Monocyte and macrophage synthesised products and behavioural evaluation could provide insight in identification as well, such as different chemokines and phagocytic activity in specific circumstances respectively.

\subsection{Closing the loop}

During the prostate cancer pilot the question arose whether monocytes could finally return to the bloodstream after performing phagocytic tasks, presumably after differentiating into macrophages ${ }^{40}$. Apart from this feature, also the 
phagocyte's ability to concentrate internalised tumour related material was investigated in the CRC-pilot ${ }^{78}$. The concentration hypothesis stated that internalisation enhanced the possibility to detect even lower levels of specific internalised materials. The travelling route of monocytes into the tissue and towards lymph nodes has been described and visualised in literature. The ability to return to the blood stream has not been properly investigated yet. One of the hypothesised routes is via the lymph nodes, through the collecting cavity, the cisterna chyli (or receptaculum chyli). The cells would then continue into the thoracic duct and right lymphatic duct and finally enter the superior vena cava via both subclavian veins. Puncture of the cisterna chyli would allow for investigating whether macrophages containing tumour related material are present. In cadaver studies the cisterna chyli could be accessed. Our group is confident that these cells will be present and detectable. Further research investigating the concentration of macrophages in the thoracic duct and the superior vena cava would be logical steps.

Secondly, through puncture of the portal vein during colorectal surgery the presence of macrophages in portal vein blood may be determined as it is the outflow from the colorectal tumour-site. In combination with central venous and peripheral venous puncture this would provide information about which location contains the highest yield of tumor-marker containing macrophages. A possible first-pass effect of the liver is hypothesised, where macrophages containing tumour-related material could extravasate. The concentration of macrophages containing tumour-related material is hypothesised to decline gradually. It is thought to be the highest in portal vein blood, then in caval blood and finally the lowest in peripheral blood. Ethical approval for performing these studies has been applied for.

Finally, a direct route has been described from the circulation towards lymph nodes using high endothelial venules. Monocytes and macrophages use that route for quick access to lymph nodes in case of infection or other intrusion. This route could be used in the opposite direction as well, as it has been shown that monocytes are able to move upstream in blood vessels during regular patrolling actions ${ }^{1}$. Performing GFP-labelling of human monocytes in vivo has not been done yet. Experimental in vivo mouse models and in vitro experiments are currently used to investigate the feasibility of fluorescent protein labelling in humans ${ }^{79}$. 


\subsection{Tumour environment research}

We investigated the presence of tumour-related material in macrophages in breast cancer and in regular breast cysts. Surprisingly enough the nonmalignant cyst-wall contained the highest number of macrophages containing tumour-related material (unpublished results). This is concordant with our observations in lymphnodes of CRC-patients. Even though not all lymph nodes had metastatic tumour cells, all lymph nodes displayed a number of macrophages containing tumour-related material. One could hypothesise that macrophages, known to ingest everything on their path, could become "acquired" or "overtaken" at a certain point by a malignant induction. This could be caused by ingested tumour related material, for instance a strand of DNA. Parts of DNA can be incorporated into the macrophages DNA and explain alternate macrophage behaviour. Lymph node metastasis based on hybrid presence has not been described before. If macrophages in lymph nodes make contact with tumour cells travelling along a lymphatic route, a hybrid cell could evolve from that contact after fusion. The ability of (hybrid) macrophages to enable hematogenous metastasis by creating the optimal environment for entrance into the blood stream ${ }^{80-82}$ may apply to (hybrid or tumour) cell passage through the lymph node as well. This would enable further lymphatic spreading. The existence of dormant hybrid cells may be an explanation for late local recurrence in breast cancer, sometimes after years of disease free survival ${ }^{83}$. These hybrids would not be attacked by a patiënt's own immune system, because they are recognised as "self". If these hybrids exist in this context, the reason for awaking from dormancy would be the next question. It could be related to accumulation of certain genetic and epigenetic traits, or a need for a certain level of specific (combined) stimulus from the stromal surrounding before metastasis.

\subsection{Macrophage-tumour cell fusion: investigating hybrids}

Hybrid cells and or macrophages orchestrate the transformation of the stromal environment by means of cytokines, matrix metalloproteinases (MMP's) and chemokines. It is thought that the macrophages exhibit a faulty M2-activation status, leading to tissue-regenerative actions. The negative effects of this altered functionality and its by-products induce environmental effects. In the beginning the immune system may be able to correct (stromal) abnormalities. When the number of abnormal cells, their inherent functioning and products increase, recuperation is less likely to occur. This results in crossing a point of no return 
and disease will eventually progress. During our research it was discovered that comparing monocyte fractions between AJCC stages I-IV in CRC patients resulted in a bell-shaped (Gaussian) graph. This implies that along the TNMstage development the immune system is initially activated, but activity is decreasing and eventually malfunctioning in activity during the later phases (unpublished results). Research focusing on the early changes during tumour and hybrid development has shown a unified way for tumour and metastases development ${ }^{17,20}$.

Tumour budding might be equal to tumour cells migrating from the primary tumour site. On the other hand the tumour cells at the edge of the tumour could also be migrating around in the vicinity of the tumour. This resembles paving a road for other tumour cells or hybrids willing to migrate, or stimulating angiogenesis. All together these observations combine interestingly. In colorectal cancer the following question arises: If the tumour budding represents (a form of) metastasis, could these "buds" be hybrid tumour-macrophage cells themselves? This hypothesis has not been postulated yet, although the diagnostic differentiation problem between budding carcinoma cells and macrophages preceding the invasive front has been described ${ }^{84}$. This finding supports this hypothesis. Research aimed at retrieving the identity or origin of the budding tumour cells needs to be initiated. Identification by an array of hybrid-specific markers would be an possibility, if only the specific markers could be identified first. Genetic profiling of these cells could therefore be an option, focussing on possible genetic markers.

\subsection{Clinical recommendations}

In oncology it is common to evaluate a patients situation by TNM staging based on tumour growth, size and invasion, nodular involvement and metastasis. If a goal is to preclude the situation that tumour growth spins out of control, attention to the patients immune system would be appropriate, especially considering the information in this thesis and recent literature. It seems that tumourigenesis always starts with immune system errors or influenced immune cells ${ }^{85,86}$. A patiënt's defence degenerates, and even worse, it evolves into a part of the offense (at least locally). If it would be possible to measure a patiënt's immunocompetence, this could enable monitoring of the ability to conduct adequate immune responses. Such a test would should be organ-specific, because (parts of) the immune system functions are differently in different organs. A multi-array assay would therefore be most appropriate. 


\section{Concluding remarks}

Our research, described in this thesis, has been conducted in the area of cancer immunology. Combined efforts between the departments of General Surgery, Clinical Pathology and Clinical Chemistry and Haematology have led to successful projects delivering potential useful diagnostic tools. Further research will investigate the position of the diagnostic tools in relation to existing modalities, the width of the concept to other types of immunological disturbances, and will need to answer marked questions from previous paragraphs. 


\section{References}

1. Auffray C, Fogg D, Garfa M, Elain G, Join-Lambert O, Kayal S, et al. Monitoring of blood vessels and tissues by a population of monocytes with patrolling behavior. Science 2007;317:666-70.

2. Bosco MC, Puppo M, Blengio F, Fraone T, Cappello P, Giovarelli M, Varesio L. Monocytes and dendritic cells in a hypoxic environment: Spotlights on chemotaxis and migration. Immunobiology 2008;213:733-49.

3. Ziegler-Heitbrock L. Monocyte subsets in man and other species. Cell Immunol 2014;289: 135-9.

4. Ziegler-Heitbrock L, Ancuta P, Crowe S, Dalod M, Grau V, Hart DN, et al. Nomenclature of monocytes and dendritic cells in blood. Blood 2010;116:e74-80.

5. Wong KL, Yeap WH, Tai JJ, Ong SM, Dang TM, Wong SC. The three human monocyte subsets: Implications for health and disease. Immunol Res 2012;53:41-57.

6. Schauer D, Starlinger P, Reiter C, Jahn N, Zajc P, Buchberger E, et al. Intermediate monocytes but not tie2-expressing monocytes are a sensitive diagnostic indicator for colorectal cancer. PLoS One 2012;7:e44450.

7. Bronsert P, Enderle-Ammour K, Bader M, Timme S, Kuehs M, Csanadi A, et al. Cancer cell invasion and EMT marker expression: A three-dimensional study of the human cancer-host interface. J Pathol 2014;234:410-22.

8. Lin EY, Li JF, Gnatovskiy L, Deng Y, Zhu L, Grzesik DA, et al. Macrophages regulate the angiogenic switch in a mouse model of breast cancer. Cancer Res 2006;66:11238-46.

9. Pollard JW. Macrophages define the invasive microenvironment in breast cancer. J Leukoc Biol 2008;84:623-30.

10. Mantovani A, Marchesi F, Porta C, Sica A, Allavena P. Inflammation and cancer: Breast cancer as a prototype. Breast 2007;16 Suppl 2:S27-33.

11. Zigmond E, Varol C, Farache J, Elmaliah E, Satpathy AT, Friedlander G, et al. Ly6C hi monocytes in the inflamed colon give rise to proinflammatory effector cells and migratory antigen-presenting cells. Immunity 2012;37:1076-90.

12. Zigmond E, Jung S. Intestinal macrophages: Well educated exceptions from the rule. Trends Immunol 2013;34:162-8.

13. van Furth R, Cohn ZA. The origin and kinetics of mononuclear phagocytes. $J$ Exp Med 1968;128:415-35.

14. Geissmann F, Auffray C, Palframan R, Wirrig C, Ciocca A, Campisi L, et al. Blood monocytes: Distinct subsets, how they relate to dendritic cells, and their possible roles in the regulation of tcell responses. Immunol Cell Biol 2008;86:398-408.

15. Joly S, Francke M, Ulbricht E, Beck S, Seeliger M, Hirrlinger $P$, et al. Cooperative phagocytes: Resident microglia and bone marrow immigrants remove dead photoreceptors in retinal lesions. Am J Pathol 2009;174:2310-23.

16. Jakubzick C, Gautier E, Gibbings S, Sojka D, Schlitzer A, Johnson T, et al. Minimal differentiation of classical monocytes as they survey steady-state tissues and transport antigen to lymph nodes. Immunity 2013;39:599-610.

17. Pawelek JM. Tumour-cell fusion as a source of myeloid traits in cancer. Lancet Oncol 2005;6:988-93.

18. Pawelek JM, Chakraborty AK. The cancer cell--leukocyte fusion theory of metastasis. Adv Cancer Res 2008;101:397-444.

19. Pawelek JM, Chakraborty AK. Fusion of tumour cells with bone marrow-derived cells: A unifying explanation for metastasis. Nat Rev Cancer 2008;8:377-86.

20. Pawelek JM. Fusion of bone marrow-derived cells with cancer cells: Metastasis as a secondary disease in cancer. Chin J Cancer 2014;33:133-9.

21. Ziegler-Heitbrock L, Hofer TP. Toward a refined definition of monocyte subsets. Front Immunol 2013;4:23. 
22. Mandl M, Schmitz S, Weber C, Hristov M. Characterization of the CD14++CD16+ monocyte population in human bone marrow. PLoS One 2014;9:e112140.

23. Burke B, Ahmad R, Staples KJ, Snowden R, Kadioglu A, Frankenberger M, et al. Increased TNF expression in CD43++ murine blood monocytes. Immunol Lett 2008;118:142-7.

24. Palframan RT, Jung $S$, Cheng $G$, Weninger W, Luo $Y$, Dorf $M$, et al. Inflammatory chemokine transport and presentation in HEV: A remote control mechanism for monocyte recruitment to lymph nodes in inflamed tissues. J Exp Med 2001;194:1361-73.

25. Zabuawala T, Taffany DA, Sharma SM, Merchant A, Adair B, Srinivasan R, et al. An ets2driven transcriptional program in tumor-associated macrophages promotes tumor metastasis. Cancer Res 2010;70:1323-33.

26. Sauter KA, Pridans C, Sehgal A, Bain CC, Scott C, Moffat L, et al. The macblue binary transgene (csf1r-gal4vp16/UAS-ECFP) provides a novel marker for visualisation of subsets of monocytes, macrophages and dendritic cells and responsiveness to CSF1 administration. PLoS One 2014;9:e105429.

27. Ojalvo LS, King W, Cox D, Pollard JW. High-density gene expression analysis of tumorassociated macrophages from mouse mammary tumors. Am J Pathol 2009;174:1048-64.

28. Coy JF, Dressler D, Wilde J, Schubert P. Mutations in the transketolase-like gene TKTL1: Clinical implications for neurodegenerative diseases, diabetes and cancer. Clin Lab 2005;51: 257-73.

29. Wittig R, Coy JF. The role of glucose metabolism and glucose-associated signalling in cancer. Perspect Medicin Chem 2008;1:64-82.

30. Xu X, Zur Hausen A, Coy JF, Löchelt M. Transketolase-like protein 1 (TKTL1) is required for rapid cell growth and full viability of human tumor cells. Int J Cancer 2009;124:1330-7.

31. Feyen O, Coy JF, Prasad V, Schierl R, Saenger J, Baum RP. EDIM-TKTL1 blood test: A noninvasive method to detect upregulated glucose metabolism in patients with malignancies. Future Oncol 2012;8:1349-59.

32. Jansen N, Coy JF. Diagnostic use of epitope detection in monocytes blood test for early detection of colon cancer metastasis. Future Oncol 2013;9:605-9.

33. Leers MP, Kolgen W, Bjorklund V, Bergman T, Tribbick G, Persson B, et al. Immunocytochemical detection and mapping of a cytokeratin 18 neo-epitope exposed during early apoptosis. $J$ Pathol 1999;187:567-72.

34. Leers MP, Bjorklund V, Bjorklund B, Jornvall H, Nap M. An immunohistochemical study of the clearance of apoptotic cellular fragments. Cell Mol Life Sci 2002;59:1358-65.

35. Makin CA, Bobrow LG, Bodmer WF. Monoclonal antibody to cytokeratin for use in routine histopathology. J Clin Pathol 1984;37:975-83.

36. Herwig R, Djavan B, Kramer G, El-Taieb MA, Kuhhas F, Leers M, Marberger M. Prostate cancer screening with a new marker based on circulating blood macrophages?. Urologe $A$ 2007;46:1066-70.

37. Herwig R. Measurement of intracellular versus extracellular prostate-specific antigen levels in peripheral macrophages: A new approach to noninvasive diagnosis of prostate cancer. Clin Prostate Cancer 2004;3:184-8.

38. Herwig R, Mitteregger D, Djavan B, Kramer G, Margreiter M, Leers MP, et al. Detecting prostate cancer by intracellular macrophage prostate-specific antigen (PSA): A more specific and sensitive marker than conventional serum total PSA. Eur J Clin Invest 2008;38:430-7.

39. Herwig R, Horninger W, Rehder P, Klocker H, Ramoner R, Thurnher M, et al. Ability of psapositive circulating macrophages to detect prostate cancer. Prostate 2005;62:290-8.

40. Leers MPG, Nap M, Herwig R, Delaere K, Nauwelaers F. Circulating psa-containing macrophages as a possible target for the detection of prostate cancer. Am J Clin Pathol 2008;129:649.

41. Nap M, ten Hoor KA, Fleuren GJ. Cross-reactivity with normal antigens in commercial anti-cea sera, used for immunohistology. The need for tissue controls and absorptions. Am J Clin Pathol 1983;79:25-31. 
42. Hübl W, Ziegler-Heitbrock LH, Bayer PM. A simple method for measurement of cd14weak cd16strong monocytes in peripheral blood. Immunobiology 2000;202:2-10.

43. Fertl A, Menzel M, Hofer TP, Morresi-Hauf A, Ziegler-Heitbrock L, Frankenberger M. Monitoring of glucocorticoid therapy by assessment of CD14(+)CD16(+) monocytes: A case report. Immunobiology 2008;213:909-16.

44. Maecker HT, Trotter J. Flow cytometry controls, instrument setup, and the determination of positivity. Cytometry A 2006;69:1037-42.

45. Wong KL, Tai JJ, Wong WC, Han H, Sem X, Yeap WH, et al. Gene expression profiling reveals the defining features of the classical, intermediate, and nonclassical human monocyte subsets. Blood 2011;118:e16-31.

46. Duffy MJ, Lamerz R, Haglund C, Nicolini A, Kalousová M, Holubec L, Sturgeon C. Tumor markers in colorectal cancer, gastric cancer and gastrointestinal stromal cancers: European group on tumor markers 2014 guidelines update. Int J Cancer 2014;134:2513-22..

47. Sturgeon CM, Duffy MJ, Stenman UH, Lilja H, Brünner N, Chan DW, et al. National academy of clinical biochemistry laboratory medicine practice guidelines for use of tumor markers in testicular, prostate, colorectal, breast, and ovarian cancers. Clin Chem 2008;54:e11-79.

48. Duffy MJ, van Dalen A, Haglund C, Hansson L, Holinski-Feder E, Klapdor R, et al. Tumour markers in colorectal cancer: European group on tumour markers (EGTM) guidelines for clinical use. Eur J Cancer 2007;43:1348-60.

49. Bökkerink GM, de Graaf EJ, Punt CJ, Nagtegaal ID, Rütten H, Nuyttens JJ, et al. The CARTS study: Chemoradiation therapy for rectal cancer in the distal rectum followed by organ-sparing transanal endoscopic microsurgery. BMC Surg 2011;11:34.

50. Colotta F, Allavena P, Sica A, Garlanda C, Mantovani A. Cancer-related inflammation, the seventh hallmark of cancer: Links to genetic instability. Carcinogenesis 2009;30:1073-81.

51. Wichmann MW, Meyer G, Adam M, Höchtlen-Vollmar W, Angele MK, Schalhorn A, et al. Detrimental immunologic effects of preoperative chemoradiotherapy in advanced rectal cancer. Dis Colon Rectum 2003;46:875-87.

52. Smits KM, Melotte V, Niessen HE, Dubois L, Oberije C, Troost EG, et al. Epigenetics in radiotherapy: Where are we heading? Radiother Oncol 2014;111:168-77.

53. Fokas E, Liersch T, Fietkau R, Hohenberger W, Beissbarth T, Hess C, et al. Tumor regression grading after preoperative chemoradiotherapy for locally advanced rectal carcinoma revisited: Updated results of the CAO/ARO/AIO-94 trial. J Clin Oncol 2014;32:1554-62.

54. Beets GL. Critical appraisal of the 'wait and see' approach in rectal cancer for clinical complete responders after chemoradiation (br J surg 2012; 99: 897-909). Br J Surg 2012;99:910.

55. Glynne-Jones R, Hughes R. Critical appraisal of the 'wait and see' approach in rectal cancer for clinical complete responders after chemoradiation. Br J Surg 2012;99:897-909.

56. Maas M, Beets-Tan RG, Lambregts DM, Lammering G, Nelemans PJ, Engelen SM, et al. Waitand-see policy for clinical complete responders after chemoradiation for rectal cancer. $J$ Clin Oncol 2011;29:4633-40.

57. Lambregts DM, Rao SX, Sassen S, Martens MH, Heijnen LA, Buijsen J, et al. MRI and diffusion-weighted MRI volumetry for identification of complete tumor responders after preoperative chemoradiotherapy in patients with rectal cancer: A bi-institutional validation study. Ann Surg 2014, Sep 10.

58. Martens MH, Lambregts DM, Kluza E, Beets-Tan RG. Tumor response to treatment: Prediction and assessment. Current Radiology Reports 2014;2:1-12.

59. van de Velde CJ, Boelens PG, Borras JM, Coebergh JW, Cervantes A, Blomqvist L, et al. EURECCA colorectal: Multidisciplinary management: European consensus conference colon \& rectum. Eur J Cancer 2014;50:1.e1-1.e34.

60. Tudyka V, Blomqvist L, Beets-Tan RG, Boelens PG, Valentini V, van de Velde CJ, et al. EURECCA consensus conference highlights about colon \& rectal cancer multidisciplinary management: The radiology experts review. Eur J Surg Oncol 2014;40:469-75.

61. Glimelius B. Neo-adjuvant radiotherapy in rectal cancer. World J Gastroenterol 2013;19: 8489-501. 
62. Vignali A, De Nardi P. Multidisciplinary treatment of rectal cancer in 2014: Where are we going? World J Gastroenterol 2014;20:11249-61.

63. Kalff V, Duong C, Drummond EG, Matthews JP, Hicks RJ. Findings on 18F-FDG PET scans after neoadjuvant chemoradiation provides prognostic stratification in patients with locally advanced rectal carcinoma subsequently treated by radical surgery. J Nucl Med 2006;47: 14-22.

64. de Geus-Oei LF, Vriens D, van Laarhoven HW, van der Graaf WT, Oyen WJ. Monitoring and predicting response to therapy with 18F-FDG PET in colorectal cancer: A systematic review. $J$ Nucl Med 2009;50 Suppl 1:43S-54S.

65. Janssen MH, Öllers MC, van Stiphout RG, Riedl RG, van den Bogaard J, Buijsen J, et al. PETbased treatment response evaluation in rectal cancer: Prediction and validation. Int $J$ Radiat Oncol Biol Phys 2012;82:871-6.

66. Guillem JG, Ruby JA, Leibold T, Akhurst TJ, Yeung HW, Gollub MJ, et al. Neither FDG-PET nor CT can distinguish between a pathological complete response and an incomplete response after neoadjuvant chemoradiation in locally advanced rectal cancer: A prospective study. Ann Surg 2013;258:289-95.

67. Janssen MH, Ollers MC, Riedl RG, van den Bogaard J, Buijsen J, van Stiphout RG, et al. Accurate prediction of pathological rectal tumor response after two weeks of preoperative radiochemotherapy using (18)f-fluorodeoxyglucose-positron emission tomography-computed tomography imaging. Int J Radiat Oncol Biol Phys 2010;77:392-9.

68. Denecke T, Rau B, Hoffmann KT, Hildebrandt B, Ruf J, Gutberlet M, et al. Comparison of CT, MRI and FDG-PET in response prediction of patients with locally advanced rectal cancer after multimodal preoperative therapy: Is there a benefit in using functional imaging? Eur Radiol 2005;15:1658-66.

69. Krauthamer M, Rouvinov K, Ariad S, Man S, Walfish S, Pinsk I, et al. A study of inflammationbased predictors of tumor response to neoadjuvant chemoradiotherapy for locally advanced rectal cancer. Oncology 2013;85:27-32.

70. Klintrup K, Mäkinen JM, Kauppila S, Väre PO, Melkko J, Tuominen H, et al. Inflammation and prognosis in colorectal cancer. Eur J Cancer 2005;41:2645-54.

71. Buijsen J, van Stiphout RG, Menheere PP, Lammering G, Lambin P. Blood biomarkers are helpful in the prediction of response to chemoradiation in rectal cancer: A prospective, hypothesis driven study on patients with locally advanced rectal cancer. Radiother Oncol 2014; 111:237-42.

72. Koelzer VH, Langer R, Zlobec I, Lugli A. Tumor budding in upper gastrointestinal carcinomas. Front Oncol 2014;4:216.

73. Baeten $\mathrm{Cl}$, Castermans K, Lammering G, Hillen F, Wouters BG, Hillen HF, et al. Effects of radiotherapy and chemotherapy on angiogenesis and leukocyte infiltration in rectal cancer. Int J Radiat Oncol Biol Phys 2006;66:1219-27.

74. Dou X, Wang RB, Yan HJ, Jiang SM, Meng XJ, Zhu KL, et al. Circulating lymphocytes as predictors of sensitivity to preoperative chemoradiotherapy in rectal cancer cases. Asian Pac $J$ Cancer Prev 2013;14:3881-5.

75. Toiyama $Y$, Inoue $Y$, Saigusa $S$, Kawamura $M$, Kawamoto $A$, Okugawa $Y$, et al. C-reactive protein as predictor of recurrence in patients with rectal cancer undergoing chemoradiotherapy followed by surgery. Anticancer Res 2013;33:5065-74.

76. Tacke F, Randolph GJ. Migratory fate and differentiation of blood monocyte subsets. Immunobiology 2006;211:609-18.

77. Paulsson JM, Held C, Jacobson SH, Lundahl J. In vivo extravasated human monocytes have an altered expression of CD16, HLA-DR, CD86, CD36 and CX(3)CR1. Scand J Immunol 2009;70:368-76.

78. Japink D, Leers MPG, Sosef MN, Nap M. CEA in activated macrophages. New diagnostic possibilities for tumor markers in early colorectal cancer. Anticancer Res 2009;29:3245.

79. Hoffman RM. Imaging cancer dynamics in vivo at the tumor and cellular level with fluorescent proteins. Clin Exp Metastasis 2009;26:345-55. 
80. Mantovani A, Schioppa T, Porta C, Allavena P, Sica A. Role of tumor-associated macrophages in tumor progression and invasion. Cancer Metastasis Rev 2006;25:315-22.

81. Lewis CE, Pollard JW. Distinct role of macrophages in different tumor microenvironments. Cancer Res 2006;66:605-12.

82. Condeelis J, Pollard JW. Macrophages: Obligate partners for tumor cell migration, invasion, and metastasis. Cell 2006;124:263-6.

83. Ignatiadis $M$, Dawson SJ. Circulating tumor cells and circulating tumor DNA for precision medicine: Dream or reality? Ann Oncol 2014;25:2304-13.

84. Satoh K, Nimura S, Hamasaki M, Aoki M, Koga K, Iwasaki H, et al. Tumor budding in colorectal carcinoma assessed by cytokeratin immunostaning and budding areas: Possible involvement of c-met. Cancer Science 2014;105:1487-95..

85. Gomez Perdiguero E, Geissmann F. Cancer immunology. Identifying the infiltrators. Science 2014;344:801-2.

86. Franklin RA, Liao W, Sarkar A, Kim MV, Bivona MR, Liu K, et al. The cellular and molecular origin of tumor-associated macrophages. Science 2014;344:921-5. 



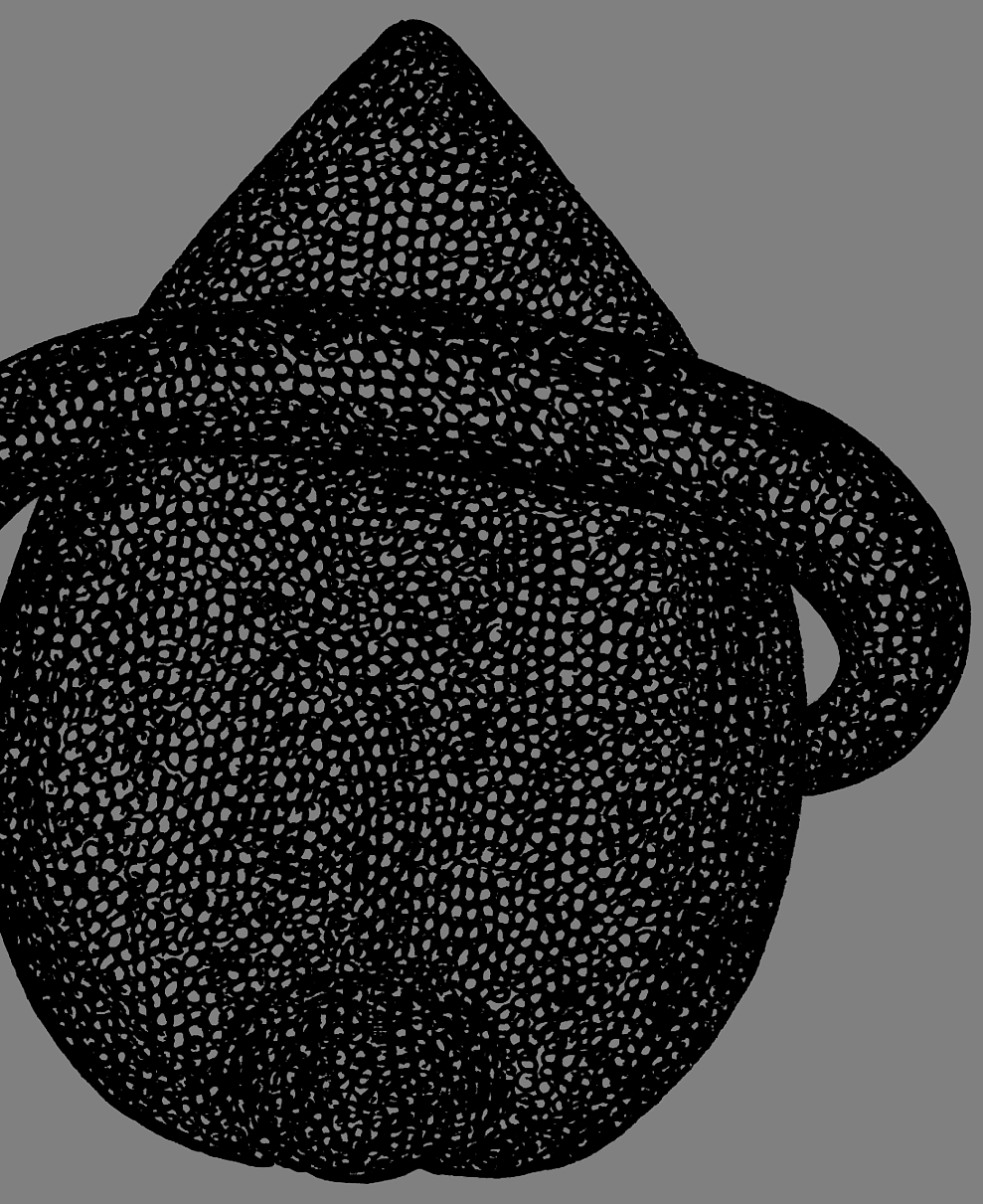

Summary 


\section{Diagnosing cancer (of the large bowel)}

\subsection{Colorectal cancer}

Tumours are composed of cellular growth that is not properly controlled by the body. In general, if a tumour grows it can develop malignant components which are potentially hazardous to the human body, eventually resulting in progression and finally in death. This thesis is focussing on cancer of the large bowel. The large bowel (the colon) consists of six parts, the cecum, the ascending colon, the transverse colon, the descending colon, the sigmoid colon and the rectal part, often called the rectum. Cancer of the large bowel or colorectal cancer (CRC) is a disease frequently diagnosed. In the total population of adults CRC is third most appearing form of cancer worldwide with over 12.000 new cases of CRC diagnosed in the Netherlands in 2012. CRC can develop as a hereditary or nonhereditary form, the latter is called sporadic CRC. Age is the number one risk factor for developing sporadic CRC, over $90 \%$ of all patients are over 50 years old. In medicine we are used to perform colonoscopies to inspect the inside of the colon and rectum, and perform biopsies of visually aberrant tissue during the procedure (Figure 8.1). These tissue samples can be investigated by a pathologist, resulting in an assessment of the presence of malignant cells. If $\mathrm{CRC}$ is diagnosed a patient will follow a predefined trajectory of investigations based on the location and the extent of the tumour. These results are discussed in a multidisciplinary team and a treatment plan, mostly including surgery, is started in accordance with the patient. After surgery a patient is put under surveillance according to CRC treatment guidelines. One of the parts of surveillance is based on a blood investigation, determining the levels of the tumour marker carcinoembryonic antigen (CEA). Since 1965 CEA is known to be present in large amounts in CRC-tissue. It has been investigated and clinically applied in CRC diagnostics and clinical management of CRC ever since. Currently it is only applied in the follow-up of patients after CRC surgery. CEA is also present in the healthy lining of the large bowel and other specific tissues in the body, but in much lower concentrations. This would allow for higher levels as being considered as disease-related. 


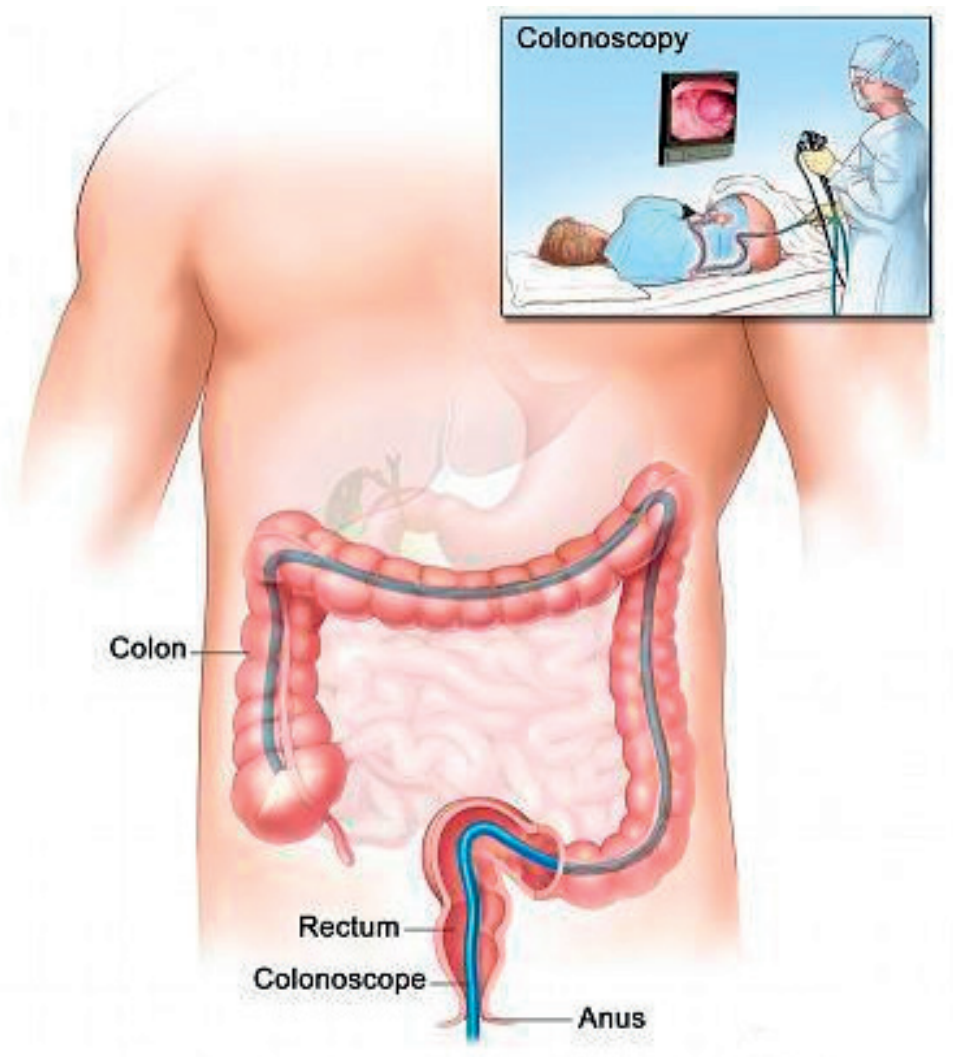

Figure 8.1 Colonoscopy: a flexible tube with a camera is introduced through the anus into the rectum, sigmoid, descending colon, transverse colon and finally through the ascending colon into the cecum. It is possible to take tissue samples of suspect lesions and to ink those lesions for future surgical resection procedures.

\subsection{CRC development}

Sporadic CRC starts developing from basal cellular changes in the colon wall, which occur during cell division. Some are genetically and some are environmentally initiated. The stages of development of CRC can be seen in Figure 8.2. 


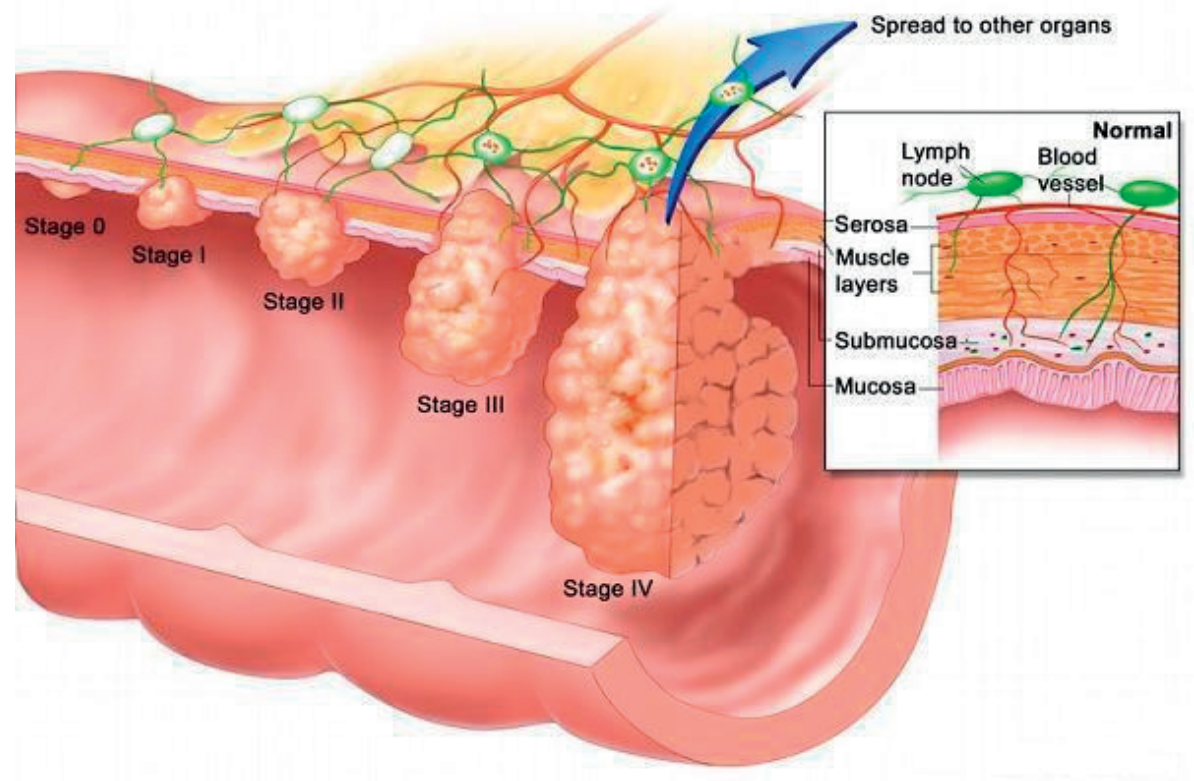

Figure 8.2 The developmental stages of colorectal cancer in the colon wall.

$\mathrm{CRC}$ can have a polyp like phenotype and grow larger and larger, or CRC starts as a flat lesion and progress towards further invading the colon wall. A mixture of both paths is also possible, when a cancerous component in the polyp head invades the colon wall through the foot of the polyp. Two examples are shown in Figure 8.3.
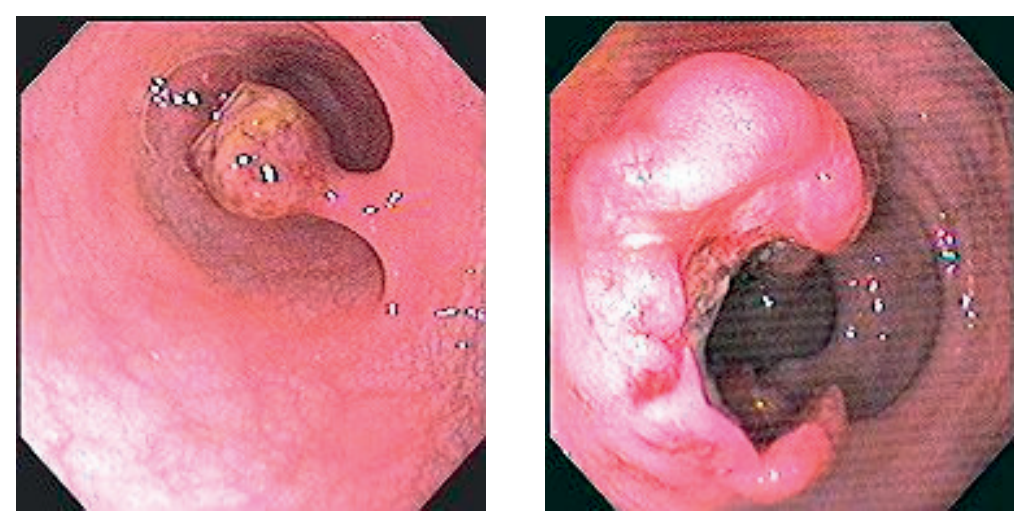

Figure 8.3 On the left side a colonic polyp and on the right side semi-circular colorectal cancer is shown. 
Curative treatment for CRC becomes more easily achievable when the diagnosis has been made in an early stage. Then it is statistically probable that no local progression or spreading throughout the body has occurred (metastasis). To diagnose CRC, a patient should present him- or herself with a recognisable pattern of complaints. Very often complaints are vague and do not immediately lead to the needed diagnosis. Proper diagnostic tools, such as imaging tools are often only applicable in advanced tumour stages. Investigating the presence of occult blood in the stool (iFOBT) can give an indication of the presence of possible tumoural development. After a positive result, a colonoscopy is always needed to confirm the diagnosis. A CRC-blood-test is not (yet) available for diagnosis, although it would potentially fill in a gap in CRCdiagnostics.

The changes in the bowel wall during CRC development can be initiated by inflammation and subsequent mutation. Faulty cell repairs to the inflamed area leading to DNA damage can cause this. This DNA damage can result in mutated cells with a possible malignant nature. Investigating this inflammatory process and the cells that cause it, can give information about how the body is resolving problems in this inflamed area.

\section{Macrophages}

To determine whether the earlier mentioned inflammation in the vicinity of tumour development, when a tumour progresses and finally during treatment of cancer could be helpful in diagnostic testing, we investigated a specific type of immune cells. These cells are called macrophages, and they are a subtype of all clean-up cells or (as these are also called) phagocytes. In "phagocyte" "phagos" means "to eat" and "cyte" means "cell". In "macrophage" "macros" means "large", combining both names ends up in "large eating cell", which indeed is one of its primary functions and describes the macrophage looks (their morphology).

Macrophages develop from other immune cells, called monocytes. These cells are produced in the bone marrow inside all long bones in the body. Monocytes and macrophages can be found in all persons, not only if you are ill. In your blood the monocytes swarm around and wherever needed they are able to go into tissue after receiving specific signals to do so. After they move into the tissue, these monocytes are able to develop macrophage looks and functions. Macrophages normally protect us from the outside world by eliminating invaders 
and instructing other immune cells how to attack these invaders, but they also attack tumour cells. Further, they have a function in wound healing, creation of blood vessels, influencing other (immune) cells and they produce various kinds of helpful substances to be used in these processes.

\subsection{Macrophages in (colorectal) cancer}

Macrophages are known to not only reside in the vicinity of a tumour, but also inside a tumour. The direct vicinity of the tumour is called the stromal environment. In both localisations, the cancer cells can influence the functions of the macrophage. Cancer cells are able to change the macrophages actions toward tumour promoting actions rather than actions resulting in tumour elimination. This means tumour cells are assisted in performing tumour progression tasks, for example alterations to the stromal environment so tumour cells are able to more easily move around. Also other tasks are known such as blood vessel formation to aid tumour growth and facilitating the entrance to the blood stream of cancer cells enabling subsequent metastasis throughout the body. Therefore the acquisition of macrophages by tumour environments can result in adverse actions of these cells, leading to disease progression rather than disease inhibition or even elimination. Macrophages do not act the same in (the vicinity) of all solid tumours. In breast cancer they are associated with poor prognosis, and in CRC their roles are not clear yet. Some researchers state that macrophages pose a risk in disease progression; others have found the macrophages able to deliver an anti-tumoural response. The distinction in the role of the macrophage in CRC has not yet been clarified. Maybe both roles exist depending on the influences they encounter. To investigate the presence of CEA in macrophages in CRC (related) tissue two studies were conducted. The first study evaluated the presence of CEA inside the macrophages in CRC tissue and lymph nodes. This was done by the use of a light microscope and applying substances that can highlight the presence of CEA in (the macrophages in) these tissues. It was found that CRC tissue contained CD68 positive cells (macrophages) being positive for CEA and that these macrophages were able to migrate into blood vessel walls (Figure 8.4 and chapter 2). Also lymph nodes were found to contain CEA-containing macrophages (Figure 8.5 and chapter 2). 

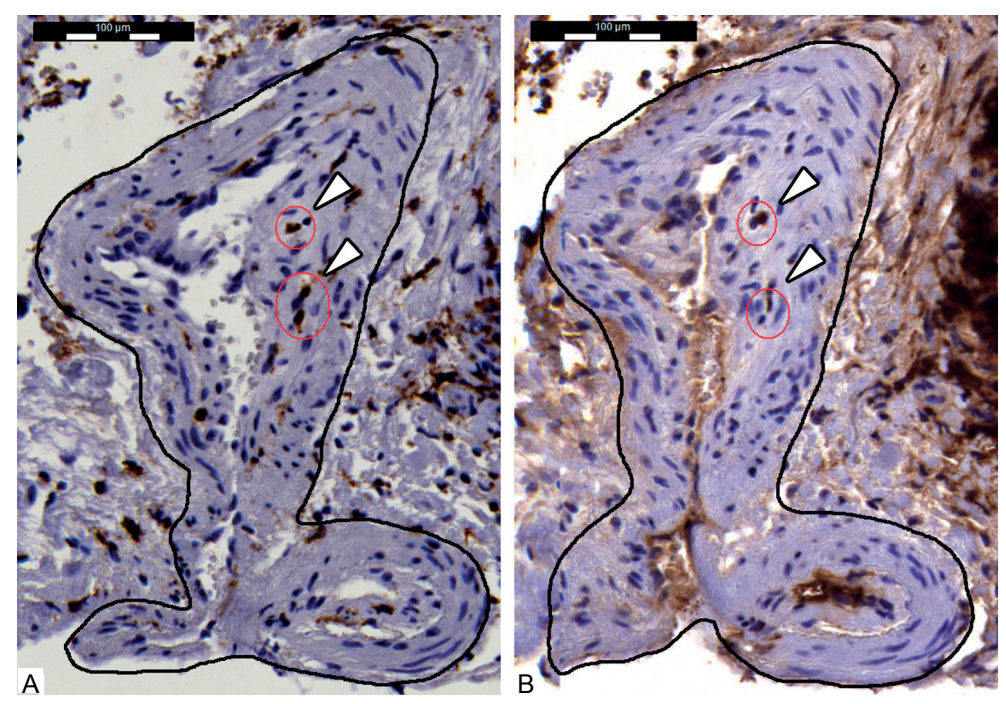

Figure 8.4 a. CD68 stain of a blood vessel (outlined) next to tumor tissue; $\mathbf{b}$. CEA stain of the same blood vessel. Intramural macrophages are present; two macrophages are positive in the CEA stain (arrowheads, circles). CEA reactivity is also visible at the luminal borders of this vessel.

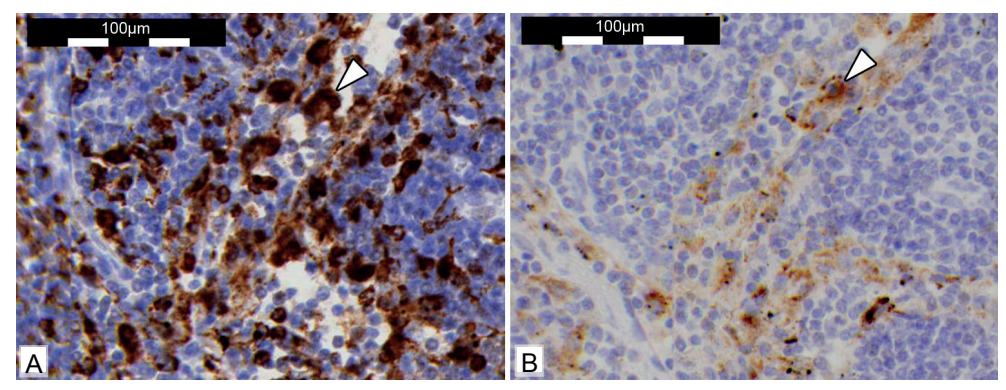

Figure 8.5 a. CD68 stain of a lymph node with widespread macrophage distribution; $\mathbf{b}$. corresponding CEA stain. CEA-containing macrophages can be seen along the lining of and are limited to the medullary sinuses. Granular staining is visible in a number of macrophages (example at arrow).

The second study was used to find out the following: Are these macrophages ingesting and digesting tumour tissue with CEA? Or are they ingesting tumour tissue and subsequently produce and present a tumour-specific marker (in this case CEA) on the macrophage-surface to signal other immune cells? As was found out, the macrophages did ingest the tumour-tissue including the CEA and that it was possible to put a staining on the ingested CEA. Using electron 
microscopy pictures were taken of macrophages containing tumour tissue with CEA present in digesting chambers called lysosomes (Figure 8.6 and chapter 3) by applying a CEA-antibody combined with a gold particle staining. This means that indeed macrophages ingest tumour-related material containing CEA. Further it means that maybe these macrophages could be detectable when investigating the blood of CRC-patients.

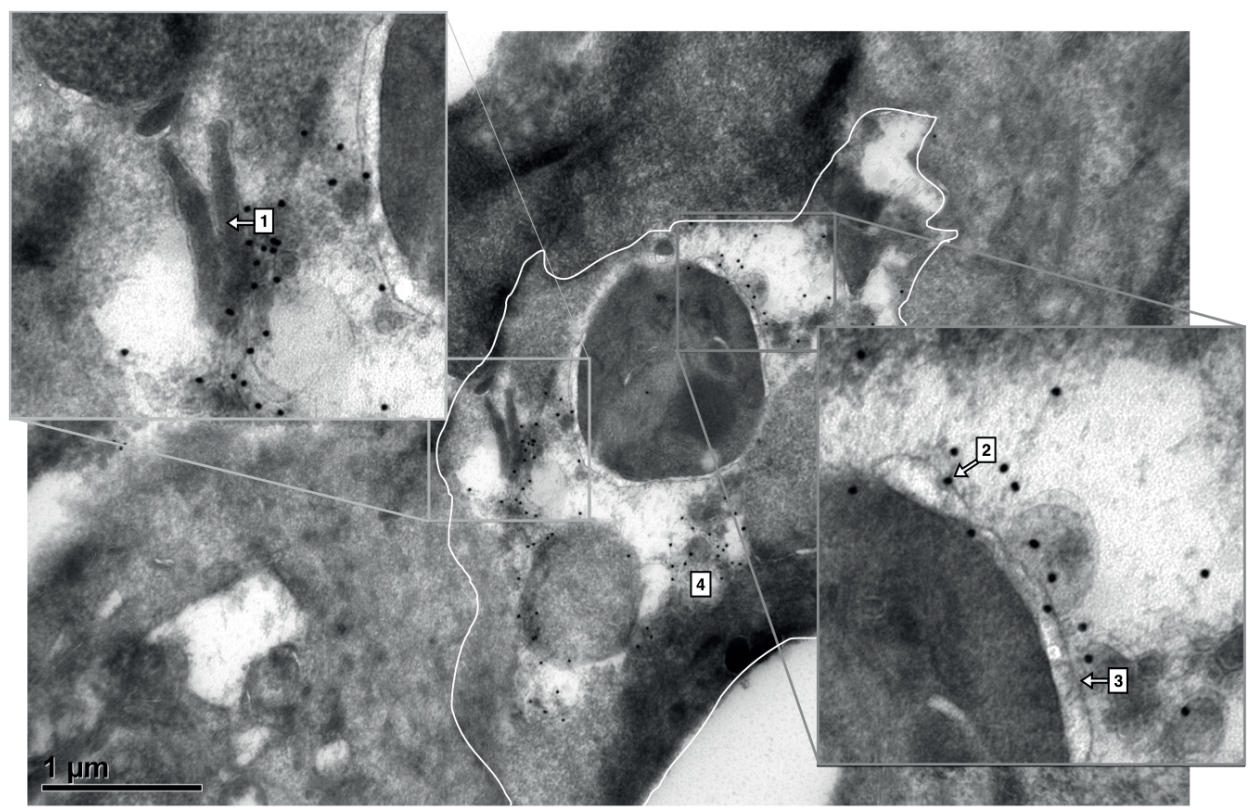

Figure 8.6 Electron-microscopy photograph showing a phagocyte (possibly a macrophage) having ingested a cell-core (lower right insert in detail) and parts of the villous membrane of a cancer cell (upper left insert). The numbers 1,2 and 3 resemble a villous part, a gold particle and a vacuole membrane respectively. Number 4 resembles part of the endoplasmic reticulum used for transport throughout the cell.

\subsection{Utilising macrophages in diagnosis of CRC}

Earlier research on prostate cancer diagnostics utilising prostate specific antigen (PSA, a tumour marker) detection in macrophages was successful in a pilot setting. Pursuing the same result with the colorectal cancer tumour marker CEA led to the development of a new diagnostic test for CRC. The new test uses a technique called flow cytometry. This technique is able to count cells from a test tube and detect specific cell characteristics (Figure 8.7).

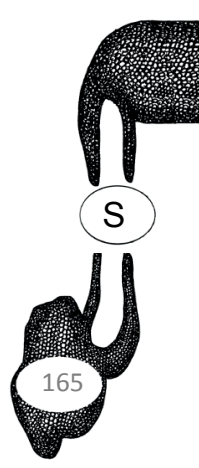




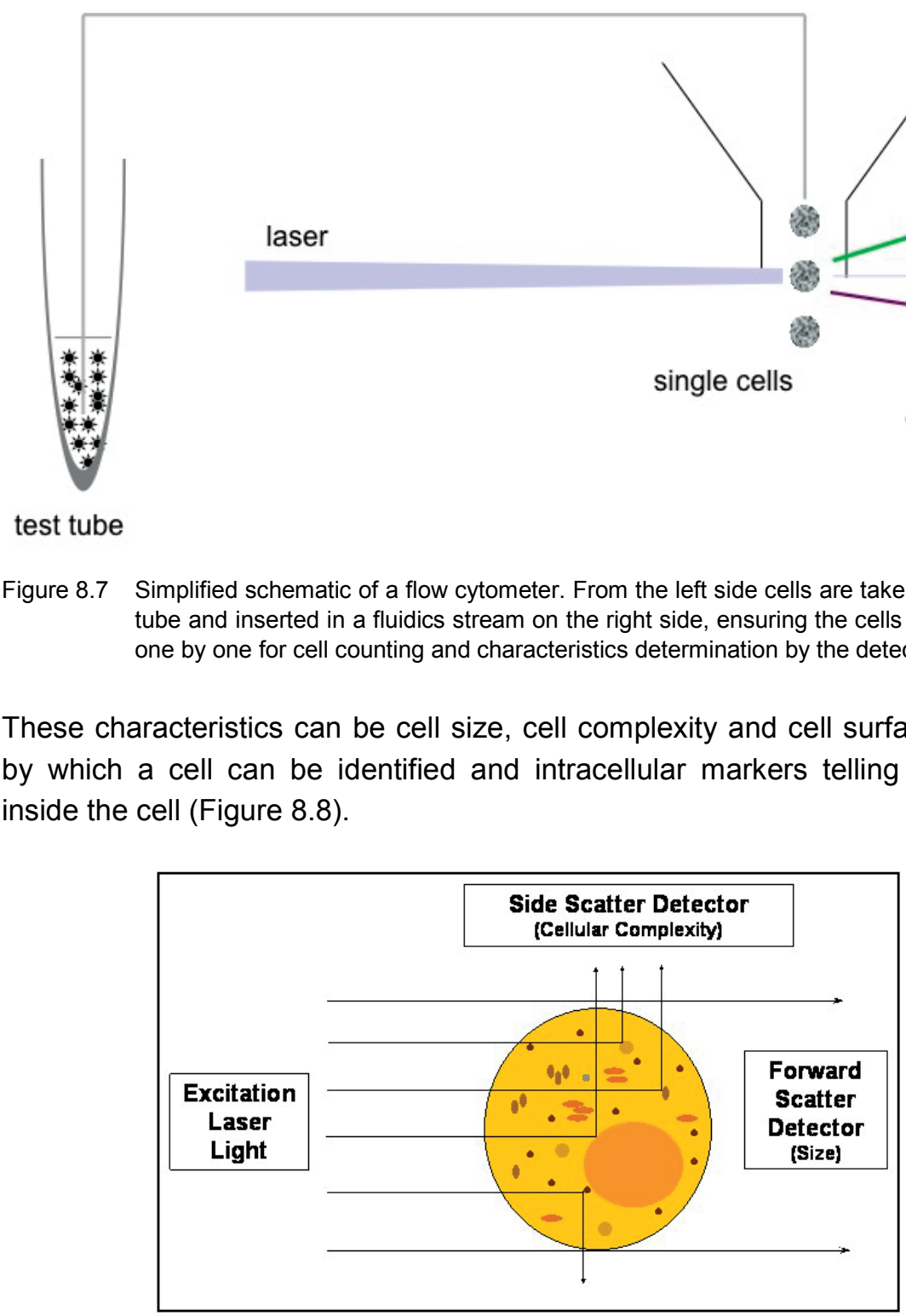

Figure 8.8 Flow cytometry is able to distinguish cell size and cell complexity by laser excitation and measuring the light that is reflected or passed by the cell. These are called side scatter and forward scatter respectively, and both can be measured by detectors in the flow cytometer. 
The cells we wanted to count were marked with specific antibodies with fluorochromes on them. Antibodies are substances that connect to specific connectors on the cell surface, called epitopes (antibodies directed against CD14 and CD16 in case of activated macrophages). Fluorochromes are substances that send out a specific colour (thus a specific frequency) of light once a laser has hit them. Allophycocyanin (APC) and Fluorescein isothiocyanate (FITC) were used for this purpose. By adding these fluorochromes to the antibodies we were able to identify the cells (activated macrophages) that could contain tumour marker epitopes we wanted to detect, by means of cell size, cell complexity and emitted light based on the presence of cell surface markers (Figure 8.9).
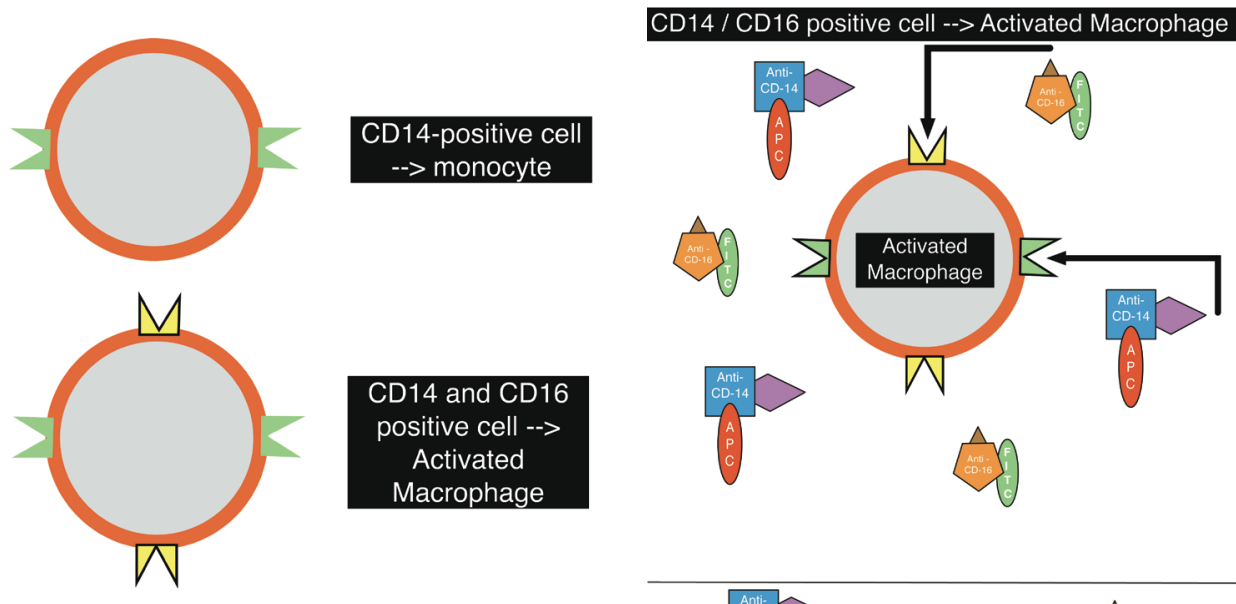

CD14 and CD16 positive cell --> Activated

Macrophage
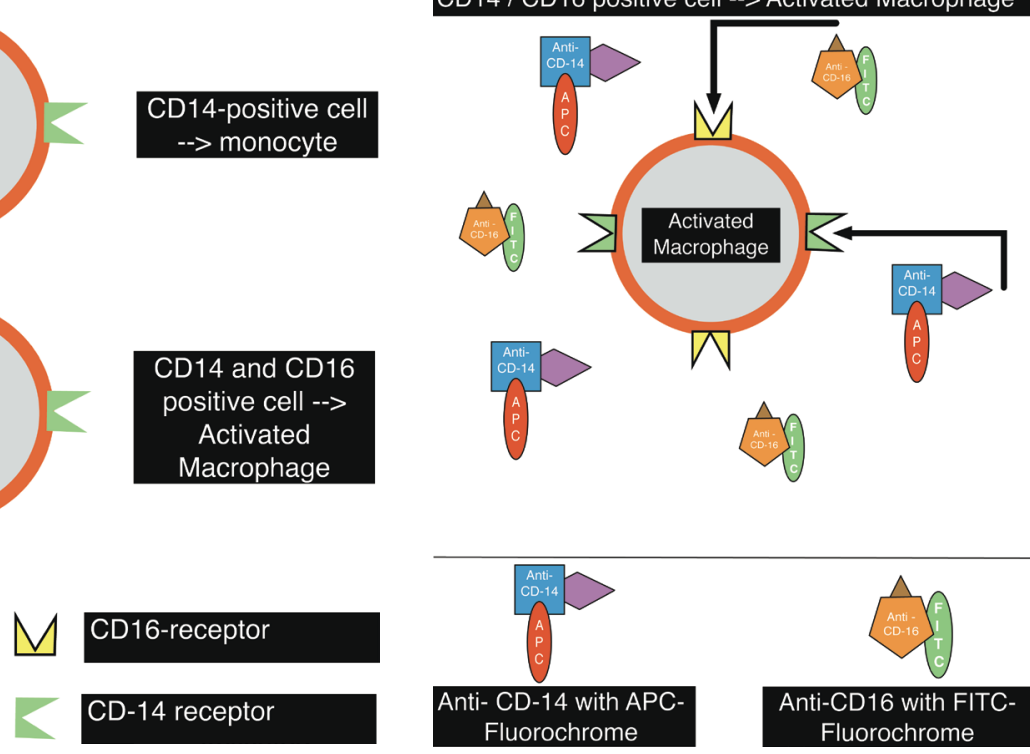

Figure 8.9 On the left side monocytes and macrophages are shown being positive for CD14 and CD14CD16 respectively. On the right side the identification of monocytes and macrophages is done by antibodies directed against CD14 and CD16 in combination with fluorochromes (APC and FITC).

After identification, the activated macrophages were treated with a substance to enable intracellular addition of antibodies directed against CEA and a fluorochrome. We used antibodies directed against CEA in combination with a third colour, phycoerythrin (PE). This enabled us to count the activated macrophages that contained CEA-positive tumour-related material (Figure 8.10). 


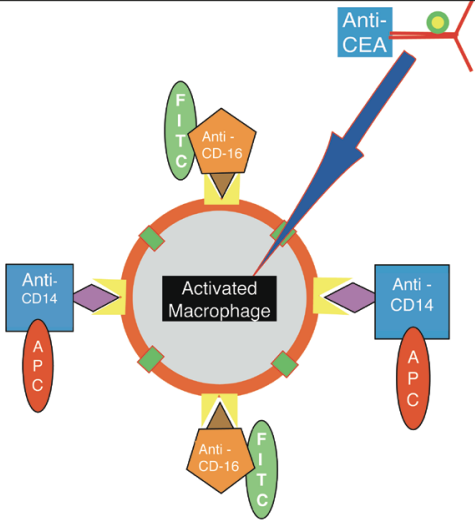
(PE)
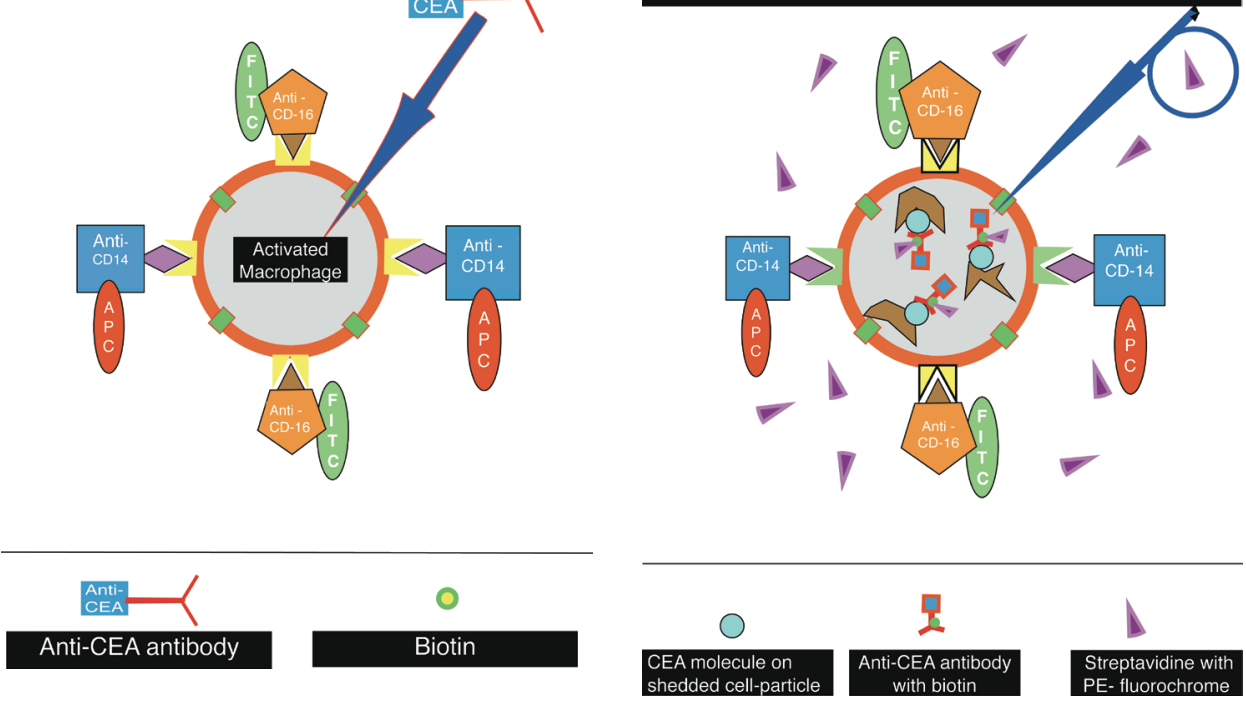

Figure 8.10 In the left figure the intracellular staining using a biotinylated antibody directed against CEA is shown after permeabilising the cell membrane. The right figure shows the addition of the PE-fluorochrome to the intracellular attached antibody directed against CEA.

This new macrophage-test was investigated in a small group of CRC patients, in benign (= non-malignant) inflammatory colon disease patients and in healthy individuals. The results showed the ability to distinguish the healthy individuals from the patients in general by the new test. When combining the new test with existing inflammation tests it was possible to distinguish the cancer patients from the benign inflammatory patients as well (Figure 8.11 and chapter 4).

Large cells with multi-lobulated nuclei being positive for CEA were photographed by investigating the used flow cytometric analysis samples prepared from peripheral blood utilising light microscopy (cytospin analysis) (Figure 8.12). These findings confirm the earlier photographed tissue macrophages findings and thereby confirm the hypothesis of macrophages with internalized CEA in colorectal cancer patients. 


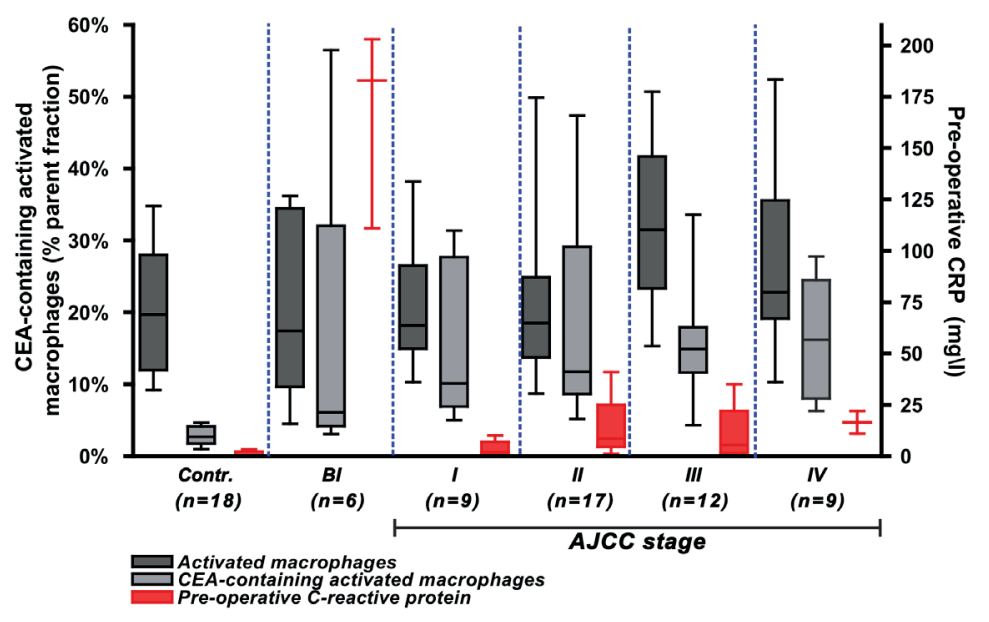

Figure 8.11 Results of the pilot study, revealing the possibility to distinguish healthy controls from benign inflammatory disease patients $(\mathrm{BI})$ and CRC patients in AJCC-stage I-IV. Combining the macrophage assay with CRP measurements enabled to distinguish even early stages of cancer from the BI-group.

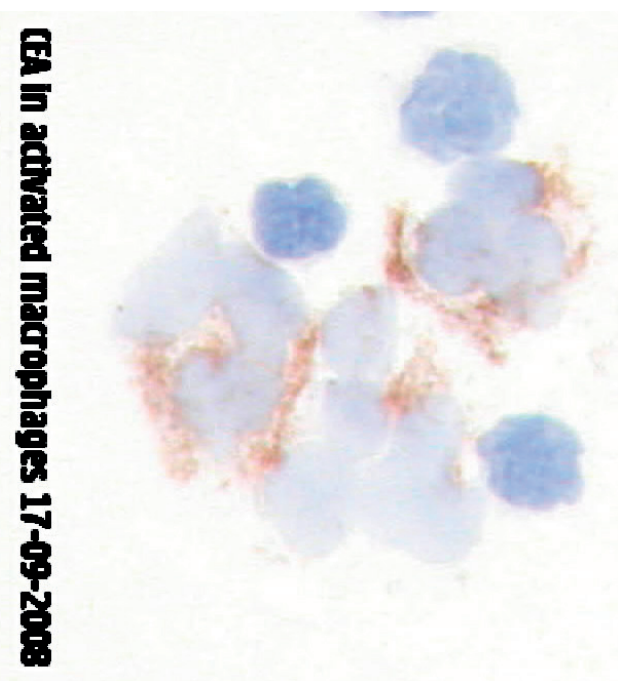

Figure 8.12 CEA in activated macrophages: The bluish colour is a core staining and the brownish colour is the staining for CEA. The CEA-staining shows a granular texture, as if the CEA remains in small pockets inside the cell. 


\subsection{Validation of the new macrophage assay}

Such a result is exciting in the beginning, but should also lead to the question if the test results are outcomes you can trust. To investigate this question, a series of tests was conducted to confirm the so-called reproducibility of this new CRC assay. Reproducibility for instance means that measuring a test tube twice, it should give the same result within a small range. Or if a test tube is measured on two different analysers, the result is the same. We also investigated the variations of monocytes and activated macrophages in healthy persons, during one month and one year (Figure 8.13). We found that the numbers of these cells do vary over time, also in healthy persons.

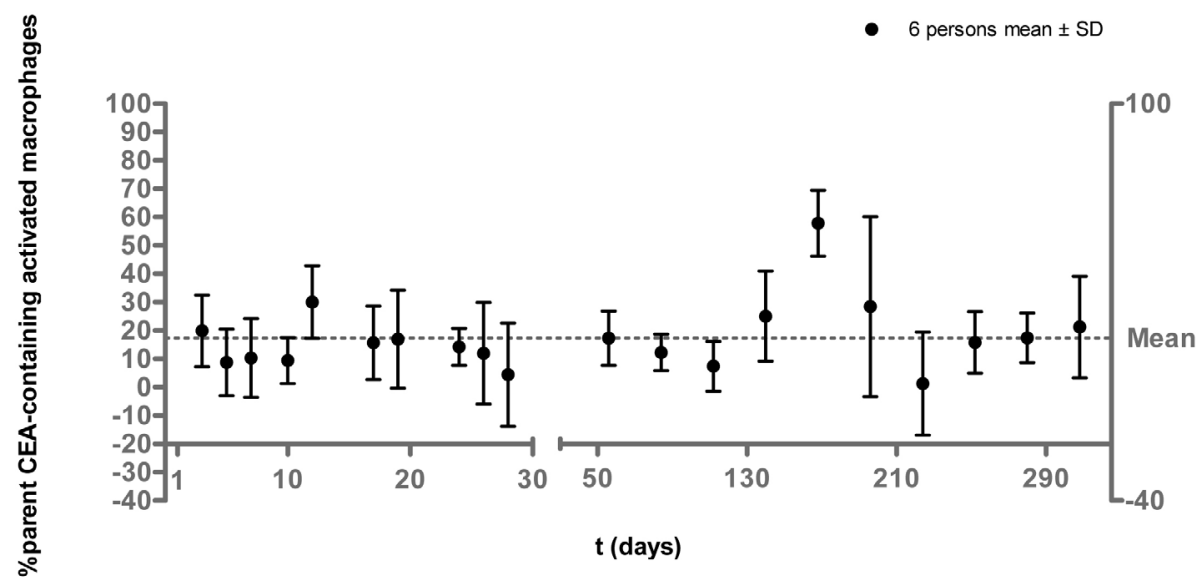

Figure 8.13 Variation of CEA-containing macrophages in 6 healthy person during one month and one year. The dotted red line shows a mean value of the six persons over this whole period.

As a result of the validation trajectory, it was found that the test was technically reproducible, but that a biological variability occurred, meaning that the patient himself causes this type of variability. This biological variability was the main source of interference with the diagnostic capabilities of the new macrophageassay (chapter 5). Therefore it was decided that the macrophage test should only be applied if another way of interpreting would be found reproducible. Another way of interpreting is currently under investigation. A change of the measured value of $25 \%$ or higher twice in a row is a new way to evaluate the 
outcomes. This interpretation option is an established way of interpreting other (serum) tumour markers. We have also investigated the macrophage test performance in alternative clinical situations.

Such alternative situations include monitoring radiochemotherapy treatment or disease progression during the follow-up period after surgery. We have investigated the treatment monitoring ability (paragraph 2.4 and chapter 6). Currently we are still performing disease monitoring during follow-up.

\subsection{Monitoring the effect of radiochemotherapy in rectal cancer}

One of the other possible applications was thought to be monitoring of neoadjuvant treatment (pre-treatment or nCRT) effects (response prediction). In rectal cancer it is common practice to perform nCRT before surgery. $\mathrm{nCRT}$ is directed at the reduction of tumour size and destroying lymph node metastasis. $\mathrm{nCRT}$ can possibly lead to downsizing the intended resection or even result in a wait and see policy when the tumour vanishes. This means a patient can benefit from predicting the response because it potentially has effect on the remaining treatment. If the prediction is that a patient would not respond at all to the regular $\mathrm{nCRT}$, an alternative treatment could be chosen including even earlier surgery. $\mathrm{nCRT}$ consists of radiotherapy in combination with chemotherapy. During the period of $\mathrm{nCRT}$ it was thought that the rectal tumours could be shedding tumour material, and that this material could be cleaned up by macrophages. To determine an additional value in predicting the effect of $\mathrm{nCRT}$ in rectal cancer, a group of rectal cancer patients before, during and after nCRT was investigated. Blood-analysis of blood drawn from rectal cancer patients during the nCRT period was performed, and PET-CT-scans of the patients were made at the same time. It was possible to see changes during the nCRT in many parameters, and some of them had promising predictive values. Adding the results of all measurements to existing response prediction models led to an increase of predictive capacity from $72 \%$ to $94 \%$ accuracy in the first two weeks of $\mathrm{nCRT}$. This means that early during the $\mathrm{nCRT}$, the treatment-team can predict with $94 \%$ certainty if the proposed treatment will have the desired effect by including the new markers. 


\subsection{Conclusion}

In conclusion, this $\mathrm{PhD}$ trajectory has focused on investigating the principle of using macrophages in a diagnostic setting in human tissue and blood, the development of a macrophage assay and the early clinical application of the macrophage assay in monitoring neoadjuvant treatment of rectal cancer patients. The assay has potential to add to the predictive capacity of response prediction models and is currently under investigation for additional value in the follow-up setting after colorectal surgery. 


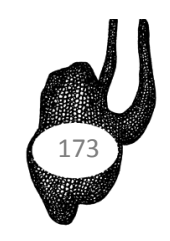





\section{Het stellen van de diagnose (darm)kanker}

\subsection{Darmkanker}

Tumoren zijn opgebouwd uit cellen die een ongecontroleerde groei hebben doorgemaakt, waarbij het lichaam niet adequaat heeft ingegrepen. In het algemeen is het zo dat, wanneer een tumor groeit, er kwaadaardige componenten in kunnen ontstaan welke in potentie bedreigend zijn voor het menselijk lichaam, resulterend in ziekteprogressie en uiteindelijk de dood. Dit proefschrift focust zich op dikke darmkanker. De dikke darm (het colon) bestaat uit zes delen: het coecum, het ascenderende (opstijgende) colon, het transverse (overstekend) colon, het descenderende (afdalende) colon, het sigmoidale colon en het rectale colon, meestal het rectum genoemd. Dikke darmkanker oftewel colorectaal carcinoom (CRC) is een frequent gediagnosticeerde ziekte. In de totale populatie van volwassenen wereldwijd is CRC de derde meest voorkomende vorm van kanker waarvan in Nederland in 2012 meer dan 12000 nieuwe gevallen werden gediagnosticeerd. Er bestaan twee vormen van CRC: een erfelijke en een niet-erfelijke vorm. De laatste vorm word ook wel het sporadisch CRC genoemd. Leeftijd is de grootste risicofactor voor het ontwikkelen van sporadisch CRC; meer dan $90 \%$ van de patiënten is boven de 50 jaar oud. In de medische wereld zijn we gewend om colonoscopieën uit te voeren om de binnenzijde van de dikke darm en het rectum te inspecteren, zodat de mogelijkheid ontstaat om een weefselhapje, een zogenaamde biopsie, van afwijkend ogend weefsel te kunnen nemen (figuur 1). Deze weefselhapjes kunnen vervolgens door een patholoog onderzocht worden, wat een analyse naar de aanwezigheid van kwaadaardige (maligne) cellen betekent. Als de diagnose CRC bij een patiënt gesteld wordt, dan zal de patiënt een voorgedefinieerd zorgpad bewandelen waarin specifieke onderzoeken worden uitgevoerd om de locatie en de uitgebreidheid van de tumor te bepalen. Deze resultaten worden multidisciplinair besproken en er wordt, na overleg met de patiënt, een behandelplan gestart, waarin een chirurgische behandeling meestal een plaats krijgt. $\mathrm{Na}$ afloop van de chirurgische interventie wordt de patiënt vervolgd in de tijd, de zogenaamde follow-up, conform de CRC behandelrichtlijnen. Een van de onderdelen van de follow-up is het bepalen van de bloed serum analyse van de tumormarker carcinoembryonic antigen, oftewel het CEA. Sinds 1965 is het bekend dat het CEA in hoge concentratie aanwezig is in CRCweefsel. Het CEA is sindsdien uitgebreid onderzocht en klinisch toegepast in het kader van CRC diagnostiek en behandeling van patiënten. Tegenwoordig wordt 
het CEA alleen nog maar toegepast in de follow-up van patiënten na CRC chirurgie. CEA is ook aanwezig in de gezonde dikke darmwand en andere specifieke weefsels in het lichaam, echter de concentratie ervan is lager. Dit geeft de optie om hogere concentraties te relateren aan de aanwezigheid van ziekte.

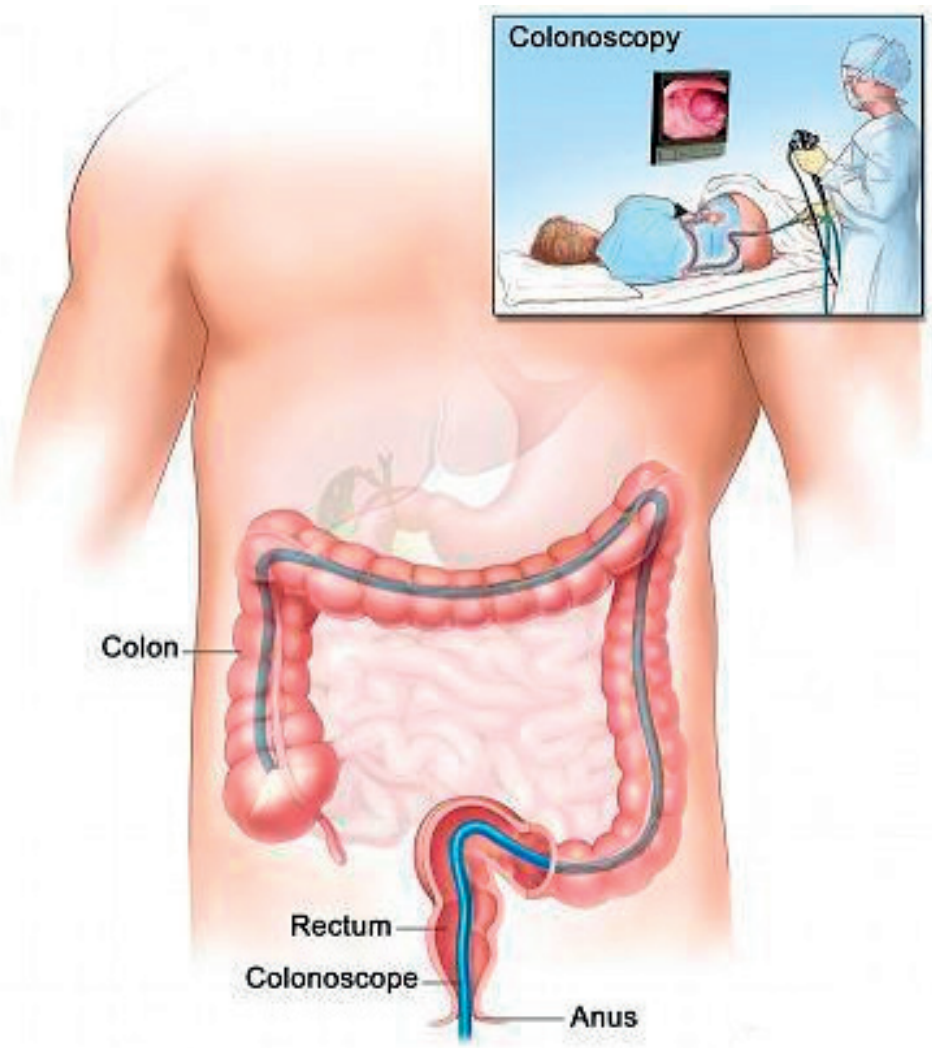

Figure 9.1 Colonoscopie: een flexibele buis met camera wordt via de anus geïntroduceerd in het rectum, door het sigmoïd, het descenderende, transverse en uiteindelijk via het ascenderende colon tot in het coecum. Het is mogelijk om weefselhapjes te nemen van verdachte laesies en om deze locaties met Oost-Indische inkt te markeren voor latere chirurgische uitname procedures.

\subsection{CRC ontwikkeling}

Sporadisch CRC begint haar ontwikkeling vanuit basale celveranderingen tijdens de celdeling in de darmwand. Sommige celveranderingen zijn genetisch bepaald en anderen zijn ontstaan onder invloed van omgevingsfactoren. De verschillende stadia van CRC zijn in figuur 2 in beeld gebracht. 


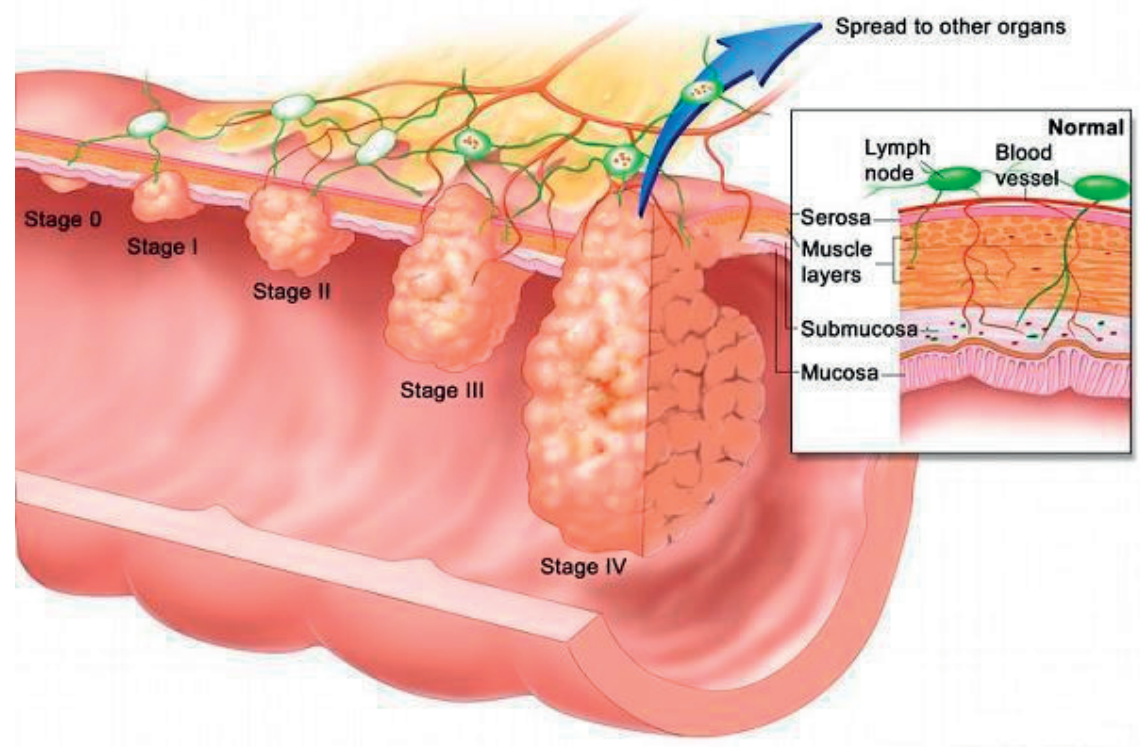

Figure 9.2 De verschillende ontwikkelstadia van CRC in de wand van de dikke darm.

CRC kan een poliep-achtig uiterlijk hebben, welke steeds groter blijft groeien. Ook kan CRC als een zogenaamde vlakke afwijking beginnen welke zich steeds verder ontwikkelt door ingroei in de darmwand. Een gemengd beeld is ook mogelijk, waarbij een kwaadaardige component in een poliepknop in de darmwand invadeert door de poliepvoet. De eerste twee voorbeelden zijn getoond in Figuur 9.3.
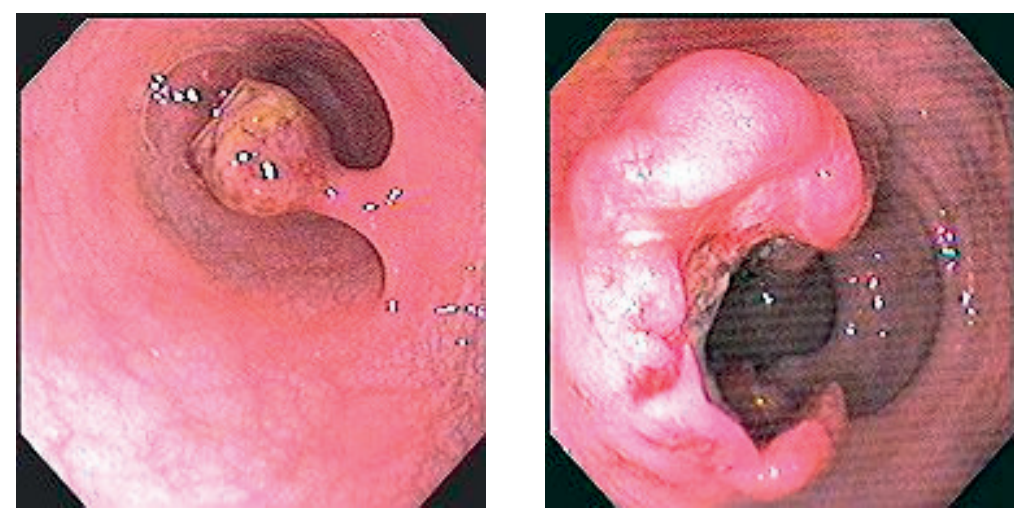

Figure 9.3 Aan de linkerzijde is een dikke darm poliep en rechts is een semi-circulaire verschijning van $\mathrm{CRC}$ gefotografeerd. 
Totale genezing van CRC wordt meer haalbaar indien de diagnose vroeg gesteld kan worden. Dan is het statistisch haalbaar om lokale doorgroei of uitzaaiingen voor te zijn. Een herkenbaar patroon van klachten draagt bij aan het stellen van de diagnose $\mathrm{CRC}$ bij een patiënt. Vaak zijn de klachten aspecifiek en leiden deze niet tot de benodigde diagnose. Adequate diagnostische methoden, zoals bijvoorbeeld beeldvorming, zijn pas toepasbaar in de meer gevorderde tumor stadia. Het onderzoek naar de aanwezigheid van occult (=onzichtbaar) bloed in de ontlasting (iFOBT) kan een indicatie geven van de aanwezigheid van een mogelijke tumorale ontaarding in de dikke darm. Bij een positief resultaat dient er altijd een colonoscopie te volgen om de diagnose te verifiëren. Een CRC-bloed test is momenteel (nog) niet beschikbaar maar zou potentieel een leemte kunnen vullen in de CRC-diagnostiek.

De veranderingen in de dikke darmwand gedurende het ontstaan van CRC kunnen het gevolg zijn van lokale ontsteking, ook wel inflammatie genoemd. Inadequate reparatie van de aangedane darmwand na de lokale inflammatie kan leiden tot DNA-schade. Deze DNA-schade kan resulteren in kwaadaardig gemuteerde cellen. Het onderzoeken van het inflammatoire proces en de bijbehorende inflammatoire cellen kunnen informatie geven over hoe het lichaam problemen oplost in ontstoken gebieden.

\section{Macrofagen}

Om te bepalen of de genoemde inflammatoire cellen van toegevoegde waarde kunnen zijn in de in ontwikkeling zijnde tumoromgeving, gedurende tumorprogressie en ook in tumordiagnostiek van toegevoegde waarde kunnen zijn, onderzochten we een specifiek type immuuncellen. Deze cellen worden macrofagen genoemd, en ze zijn een subtype van alle opruimcellen, ook wel fagocyten genoemd. In "fagocyt" betekent "fagos" "eten" en "cyt" betekent "cel". In "macrofaag" betekent "macro" "groot", en gecombineerd leidt dat tot "grote eetcel", wat inderdaad een van de primaire functies is en ook het uiterlijk (de morfologie) van de macrofaag beschrijft.

Macrofagen ontwikkelen zich vanuit andere immuuncellen, de zogenaamde monocyten. Deze cellen ontstaan in het beenmerg wat aanwezig is in alle lange pijpbeenderen in het lichaam. Monocyten en macrofagen zijn in alle mensen aanwezig, ook in gezonde toestand. Monocyten zwermen rond in de bloedbaan en waar nodig zijn ze in staat het weefsel binnen te dringen indien specifieke signalen daarvoor aanwezig zijn. 
$\mathrm{Na}$ het binnendringen in het weefsel zijn de monocyten in staat het uiterlijk en de functionaliteit van macrofagen aan te nemen. Macrofagen beschermen ons normaliter voor de buitenwereld door directe eliminatie van binnendringende ziektekiemen en het dirigeren van ander immuuncellen in het aanvallen van de binnendringers, maar ze vallen ook tumorcellen aan. Verder hebben ze een rol in wondheling, de aanleg van bloedvaten, het beïnvloeden van andere (immuun)cellen en produceren ze vele relevante stoffen die benodigd zijn voor de uitvoer van voornoemde taken.

\subsection{Macrofagen in (colorectaal) carcinoom}

Van macrofagen is bekend dat ze zich niet alleen in de nabijheid van een tumor ophouden, maar ook aanwezig zijn binnen in een tumor. De directe omgeving van een tumor wordt ook wel het stromale weefsel genoemd. In beide lokalisaties kunnen de functies van macrofagen beïnvloed worden door de kankercellen. Kankercellen zijn in staat de macrofagenfuncties richting tumorcel progressie te sturen in plaats van tumorcel eliminatie. Dit betekent dat tumorcellen geholpen worden in de taak van tumor progressie, bijvoorbeeld door het stromale weefsel dusdanig te veranderen van samenstelling dat tumorcellen er gemakkelijk doorheen kunnen bewegen. Ook andere taken worden beïnvloed, zoals bloedvatformatie ten behoeve van tumorgroei en het faciliteren van toegang tot de bloedbaan voor de kankercellen; deze aanpassingen maken de vorming van uitzaaiingen (metastasering) mogelijk.

Het aantrekken van macrofagen richting tumor omgevingen kan daarom leiden tot tegengestelde acties van deze cellen, wat vervolgens leidt tot ziekteprogressie in plaats van het inhiberen of elimineren van de ziekte. Macrofagen gedragen zich niet hetzelfde in (de omgeving van) alle vaste (solide) tumoren. In borstkanker worden ze geassocieerd met een slechtere prognose, maar in CRC zijn hun rollen nog niet geheel duidelijk. Sommige onderzoekers stellen dat macrofagen een risico vormen op ziekte progressie, anderen hebben gesteld dat macrofagen in staat zijn om een antitumorale reactie te leveren.

Het onderscheid in de verschillende rollen van de macrofaag in CRC is nog niet gemaakt. Misschien bestaan beide rollen simultaan afhankelijk van de stimuli waaraan ze bloot gesteld worden. Om de aanwezigheid van CEA in macrofagen in CRC (geassocieerd) weefsel te onderzoeken werden twee studies uitgevoerd. De eerste studie evalueerde de aanwezigheid van CEA in macrofagen en lymfeknopen. Dit werd gedaan middels het toevoegen van substanties die CEA in (macrofagen in) het weefsel konden aantonen, gebruikmakende van 
lichtmicroscopie. Er werden CD68 positieve cellen (macrofagen) in het CRC weefsel gevonden, die ook positief waren voor CEA. Tevens bleek dat deze macrofagen in staat waren om in de wand van bloedvaten te migreren (Figuur 9.4 en hoofdstuk 2). Er werden ook lymfeknopen gevonden die positief waren voor CEA-bevattende macrofagen (Figuur 9.5 en hoofdstuk 2).
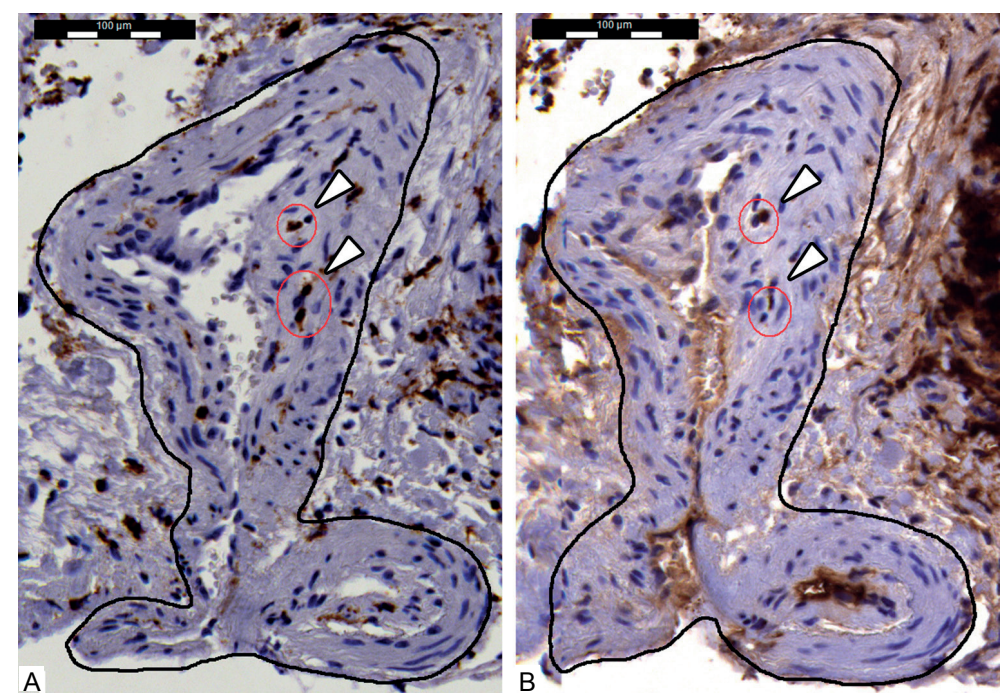

Figure 9.4 a. CD68 kleuring van een bloedvat (omlijnd) liggend in tumorweefsel. b. CEA kleuring van het zelfde bloedvat, in de wand van het bloedvat (intramuraal) zijn 2 macrofagen aanwezig die CEA-positief zijn (omcirkeld met pijlhoofdjes erbij). CEA reactiviteit is ook zichtbaar aan de binnenzijde (luminale zijde) van het bloedvat.
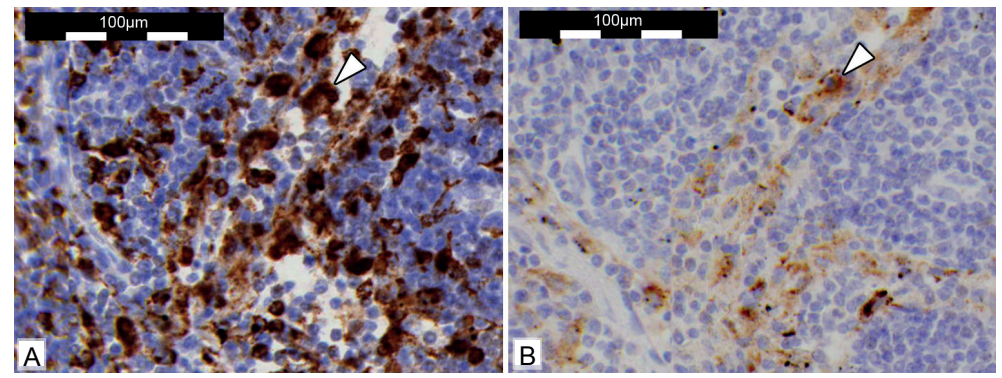

Figure 9.5 a. CD68 kleuring van een lymfeklier met uitgebreide macrofagen distributie; b.. bijbehorende CEA-kleuring. CEA-bevattende macrofagen kunnen herkend worden langs de belijning van en zijn beperkt tot de medullaire sinussen van de lymfeklier. Granulaire kleuring is herkenbaar in meerdere macrofagen (zie pijlhoofd). 
De tweede studie werd uitgevoerd om de volgende vraag te beantwoorden: nemen macrofagen tumorweefsel met CEA op en verteren ze dat? Of nemen ze tumorweefsel op en produceren en presenteren ze vervolgens een tumorspecifieke marker (in dit geval CEA) op het macrofagen oppervlak met als doel om andere immuuncellen te informeren? De studie wees uit dat macrofagen inderdaad tumorweefsel inclusief CEA opnamen en dit verteerden, en dat het mogelijk was om het opgenomen CEA aan te kleuren. Door middel van elektronen microscopie werden foto's gemaakt van macrofagen die tumorweefsel inclusief CEA hadden opgenomen in speciale verteringskamers, zogenaamde lysosomen (Figuur 9.6 en hoofdstuk 3), door het toepassen van CEA-antilichamen in combinatie met een goud-bolletjes kleuring. Dit betekent dat macrofagen inderdaad CEA-bevattend tumor-gerelateerd materiaal opnemen. Verder betekend dit dat deze macrofagen misschien aantoonbaar zijn in het bloed van patiënten met colorectaal carcinoom.

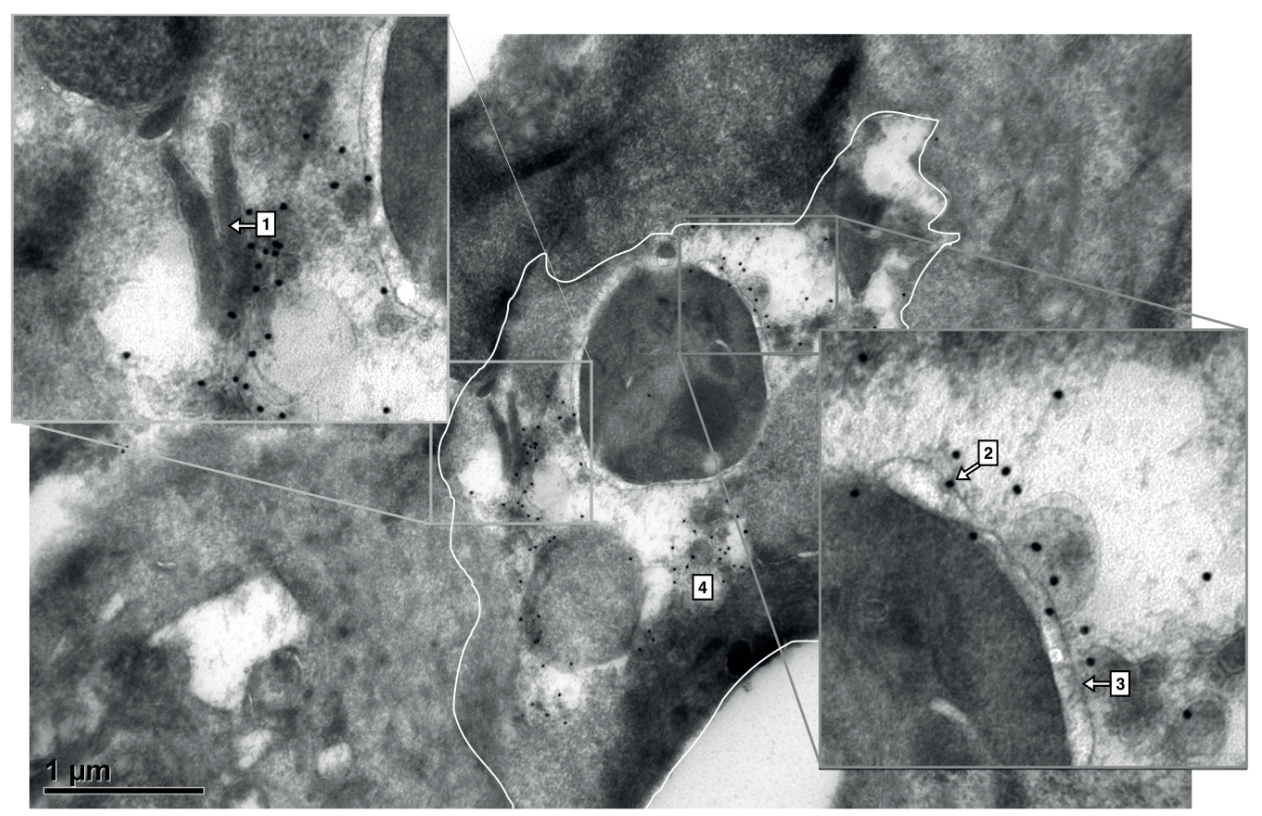

Figure 9.6 Elektronenmicroscopie foto waarin een fagocyt zichtbaar is, (mogelijk een macrofaag) na het opnemen van een celkern (inzet rechtsonder in detail) en delen van het villeuze membraan van een kankercel (inzet linksboven). De nummers 1, 2 en 3 staan voor een villeus membraandeel, een goudbolletje en een membraan van een vacuole). Nummer 4 geeft een deel van het endoplasmatisch reticulum weer, bedoeld voor transport door de cel.

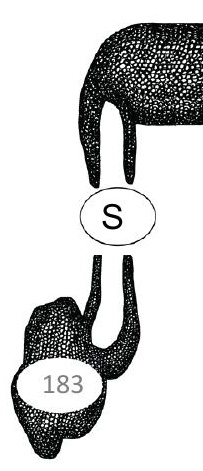




\subsection{De toepassing van macrofagen in de diagnostisering van CRC}

Eerder experimenteel onderzoek gericht op prostaat kanker diagnostiek waarbij gekeken werd naar het detecteren van prostaat specifiek antigen (PSA, een tumormarker) in macrofagen bleek succesvol. Het nastreven van hetzelfde resultaat bij CRC met de tumormarker CEA leidde tot de ontwikkeling van een nieuwe diagnostische test voor CRC. De nieuwe test maakt gebruik van een techniek welke flowcytometrie genoemd wordt. Deze techniek is in staat cellen te tellen in een testbuisje en detecteert specifieke cel karakteristieken.

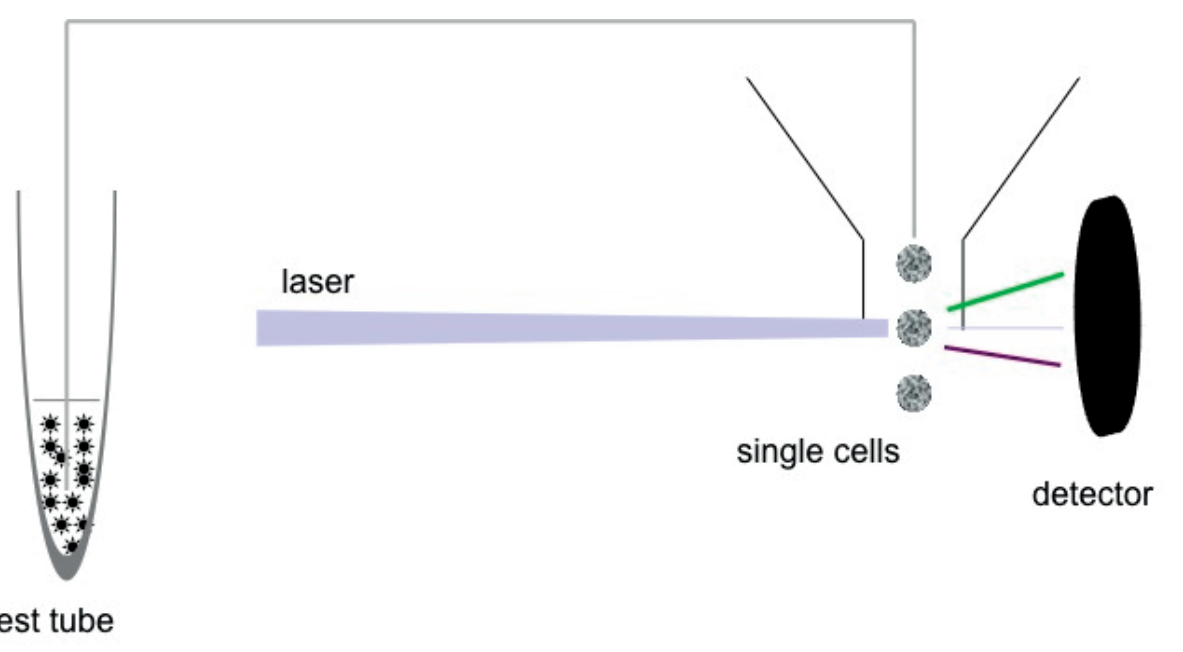

Figure 9.7 Een versimpeld schema van een flowcytometer. Vanaf de testbuis links in het schema worden cellen opgezogen en in een vloeistofstroom geïnjecteerd aan de rechterzijde van het schema, wat ervoor zorgt dat de cellen één voor één een laser passeren om de cellen te tellen en de cel-karakteristieken te bepalen doormiddel van detectoren die het laserlicht opvangen.

Deze karakteristieken kunnen celgrootte, cel-complexiteit, cel-oppervlakte markers ter identificatie van de cellen, en intracellulaire markers welke de inhoud van de cel beschrijven inhouden. 


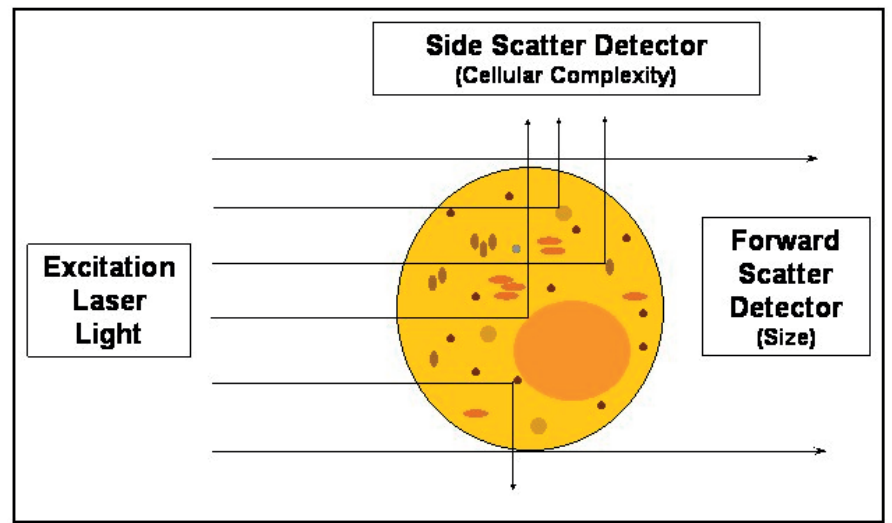

Figure 8.8 Flowcytometrie is in staat celgrootte en cel-complexiteit te onderscheiden doormiddel van het meten van het laserlicht wat gereflecteerd en doorgelaten wordt . Deze parameters worden respectievelijk Side scatter oftewel "zijwaartse strooiing" en Forward scatter oftewel "voorwaartse strooiing' genoemd; beide kunnen gemeten worden door de detectoren in de flowcytometer.

De cellen welke geteld dienden te worden werden gemarkeerd met specifieke antilichamen met fluorochromen. Antistoffen zijn stoffen welke kunnen aangrijpen op specifieke aangrijpingspunten op het celoppervlak, ook wel epitopen genoemd (anti-CD14 en anti-CD16 in het geval van geactiveerde macrofagen). Fluorochromen zijn stoffen die in staat zijn een specifieke kleur (een specifieke frequentie) licht uit te zenden na aanstralen middels de laser. Allophycocyanin (APC) en Fluorescein isothiocyanate (FITC) werden gebruikt voor dit doeleinde. Het toevoegen van deze fluorochromen aan de antilichamen stelde ons in staat de cellen (in dit geval de geactiveerde macrofagen) welke tumormerkstoffen zouden kunnen bevatten, te identificeren, doormiddel van de celgrootte, de cel-complexiteit en het uitgezonden licht op basis van de oppervlaktemarkers (Figuur 9.9).

$\mathrm{Na}$ identificatie werden de geactiveerde macrofagen behandeld met een substantie die intracellulaire kleuring met een antilichaam tegen CEA mogelijk maakte, waarna tevens de toevoeging van een fluorochroom mogelijk was. $\mathrm{Er}$ is gebruik gemaakt van een antilichaam tegen CEA in combinatie met een derde kleur, phycoerythrin (PE). Deze stap maakte het mogelijk om de geactiveerde macrofagen te tellen die CEA-positief tumorgerelateerd materiaal bevatten (Figuur 9.10).

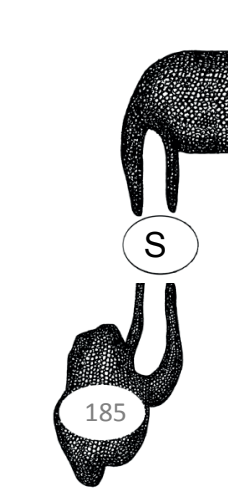




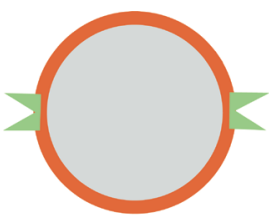

\section{CD14-positive cell $\rightarrow$ monocyte}

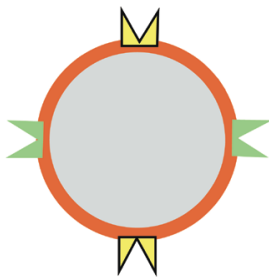

\section{CD14 and CD16 \\ positive cell --> \\ Activated \\ Macrophage}

\section{$M$ CD16-receptor}

CD-14 receptor
CD14 / CD16 positive cell --> Activated Macrophage
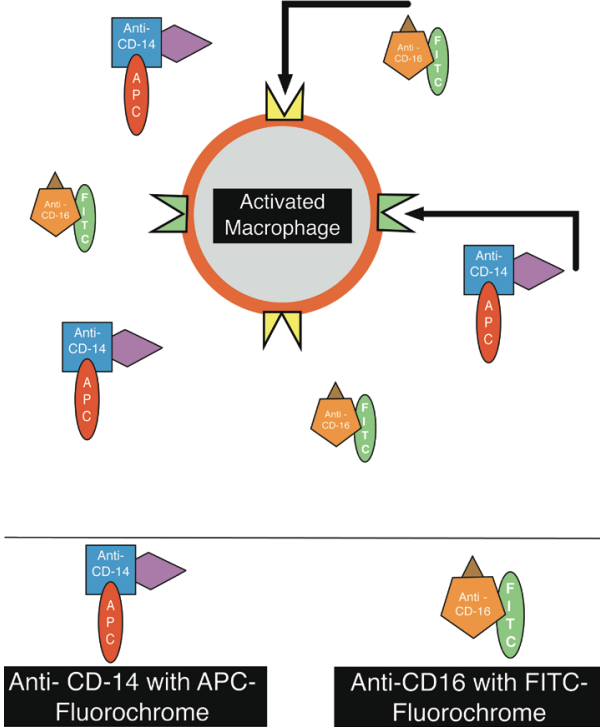

Figure 9.9 Aan de linkerzijde worden monocyten en macrofagen getoond welke respectievelijk positief zijn voor CD14 en CD14CD16. Aan de rechterzijde wordt de identificatie getoond van monocyten en macrofagen op basis van antilichamen gericht tegen CD14 en CD16 in combinatie met fluorochromen (APC en FITC).
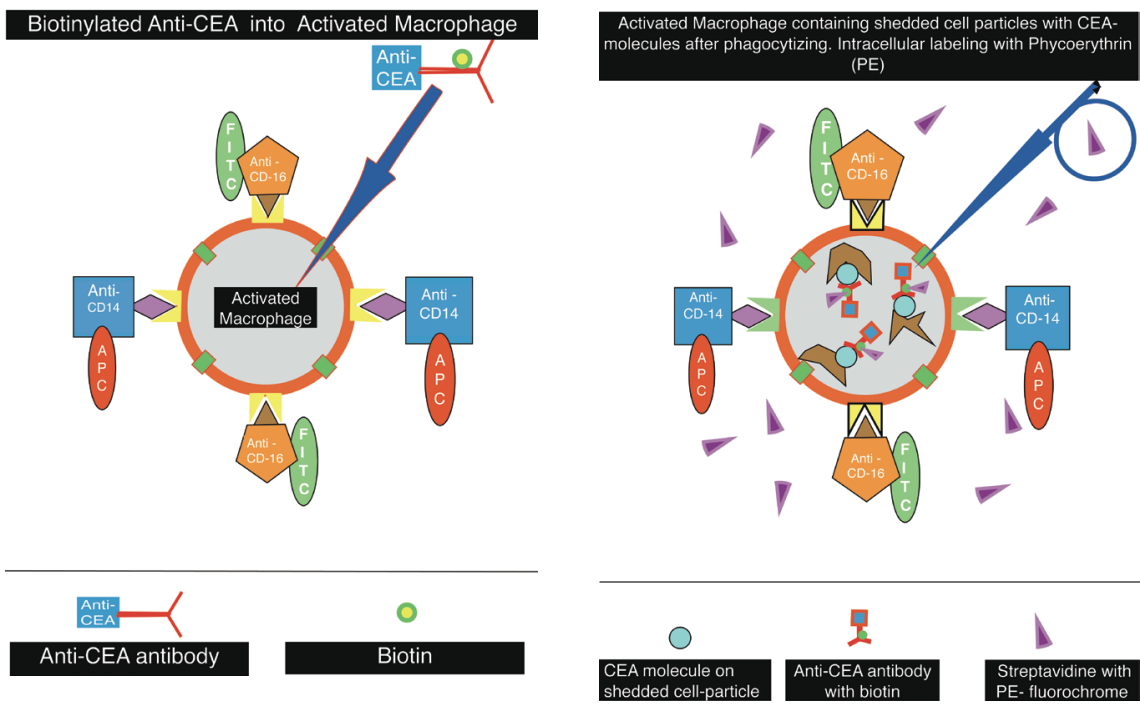

Figure 9.10 In het linker figuur wordt de intracellulaire kleuring getoond middels gebiotynileerd antilichaam gericht tegen CEA na het doorgankelijk maken van het celmembraan. Het rechter figuur toont de toevoeging van het PE-fluorochroom aan het intracellulair gekoppelde antilichaam tegen CEA. 
Deze nieuwe macrofagen-test werd onderzocht in een kleine groep CRCpatiënten, bij patiënten met goedaardige (benigne) darmaandoeningen en bij gezonde individuen. De resultaten lieten de mogelijkheid zien om de gezonde individuen te onderscheiden van de totale patiëntengroep. Na combinatie van de nieuwe macrofagen test met een bestaande inflammatietest bleek het mogelijk om de kankerpatiënten te onderscheiden van de patiënten met benigne inflammatoire darmziekte (Figuur 9.11 en hoofdstuk 4).

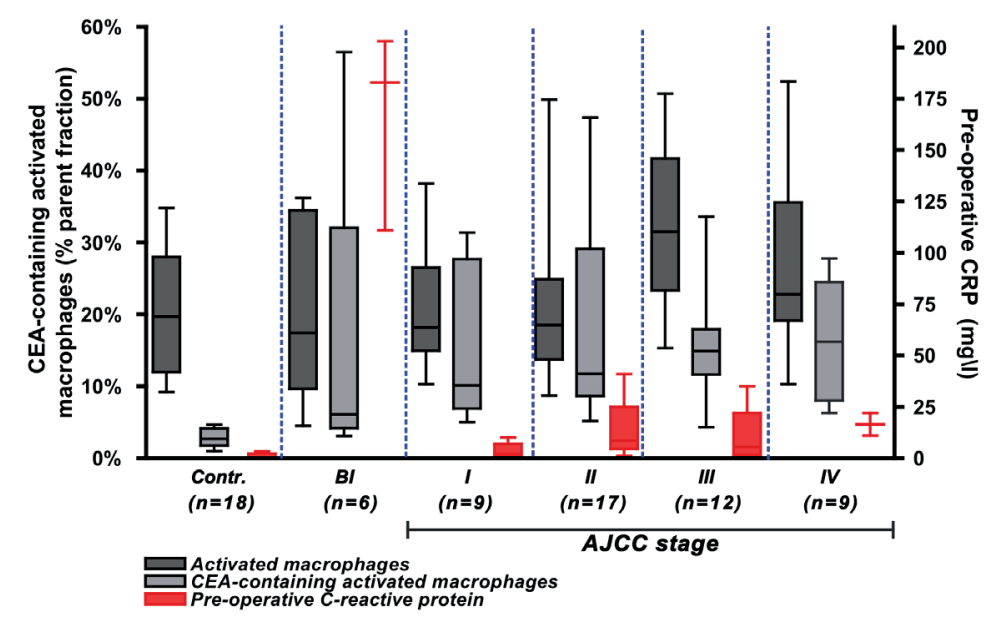

Figure 9.11 De resultaten van de proefstudie, welke laat zien dat er onderscheid gemaakt kan worden tussen de gezonde individuen, benigne darmziekte patienten (BI) en de CRC patienten in de stadia I-IV. Na het combineren van de macrofagen-test met CRP metingen blijkt het mogelijk om ook de vroege CRC-stadia te onderscheiden van de $\mathrm{BI}$ groep.

Uit flowcytometrische analyse buisjes, die vanuit perifeer afgenomen bloed waren opgewerkt, werden grote cellen met meerlobbige kernen geëxtraheerd, welke positief bleken voor CEA. Middels lichtmicroscopie (cytospin-analyse) konden deze cellen gefotografeerd worden (Figuur 9.12). Deze bevindingen bevestigen het beeld van de eerder gefotografeerde weefsel macrofagen en daardoor wordt de hypothese van macrofagen met geïnternaliseerd CEA bevestigd. 


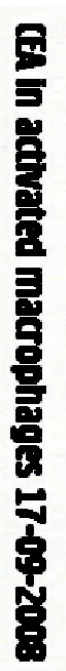

Figure 9.12 CEA in geactiveerde macrofagen: De blauwachtige kleur is een kernkleuring en de bruinachtige kleur is de CEA kleuring. De CEA-kleuring laat een korrelige (granulaire) textuur zien, alsof het CEA zich in kleine holtes in de cel bevindt.

\subsection{Validatie van de nieuwe macrofagen test}

Een resultaat zoals hierboven beschreven leidt tot enthousiasme, maar dient ook te leiden tot de vraag of de uitkomsten betrouwbaar zijn. Om deze vraag te beantwoorden werd een serie testen uitgevoerd om de zogenaamde reproduceerbaarheid te onderzoeken. Reproduceerbaarheid betekent bijvoorbeeld dat het twee keer meten van één enkele analysebuis een gelijk resultaat geeft binnen bepaalde minimale marges. Tevens dient het zo te zijn dat wanneer één analysebuisje op twee verschillende analyseapparaten gemeten wordt de uitkomsten overeenkomen. De variatie van monocyten en geactiveerde macrofagen bij gezonde proefpersonen werd gemeten gedurende één maand en één jaar (Figuur 9.9). We concludeerden dat de aantallen van de voornoemde cellen varieerden in de tijd, ook in gezonde proefpersonen. 


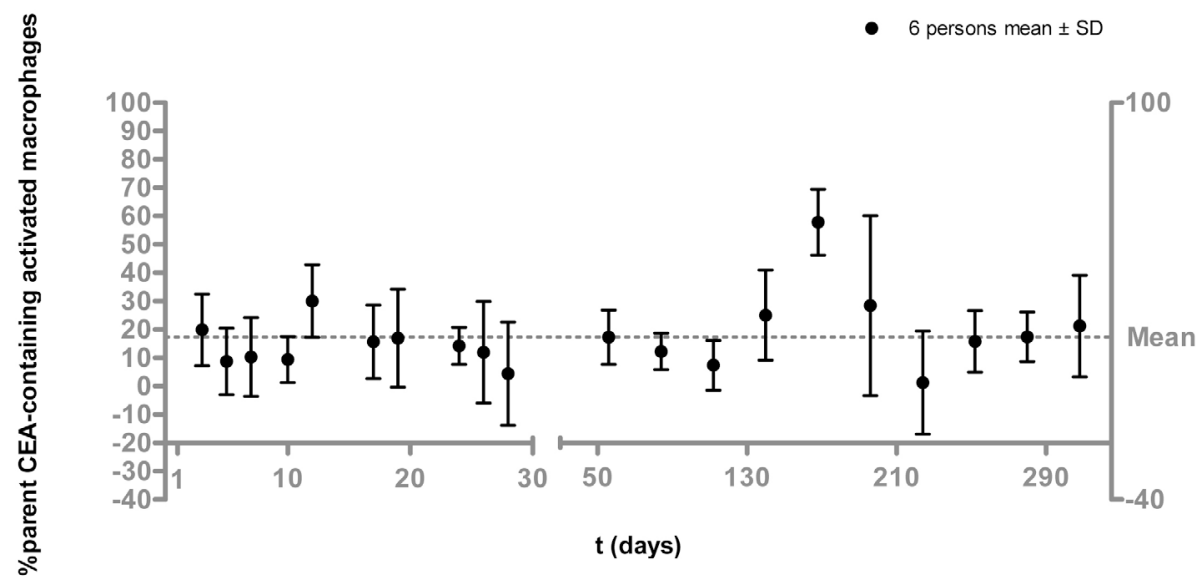

Figure 9.13 Variatie van de CEA-bevattende macrofagen in zes gezonde proefpersonen gedurende één maand en gedurende één jaar. De rode stippellijn laat de gemiddelde waarde van de zes gezonde proefpersonen over de hele periode van één jaar zien.

Het validatietraject resulteerde in de conclusie dat de macrofagentest technisch reproduceerbaar was, maar dat biologische variatie optrad, wat betekent dat de patiënt zelf de variatie veroorzaakt. Deze biologische variatie bleek de grootste bron van verstoring te zijn ten opzichte van de diagnostische capaciteiten van de nieuwe macrofagen test (hoofdstuk 5). Zodoende werd besloten dat de macrofagen test alleen toegepast kon worden indien een andere wijze van interpretatie als reproduceerbaar aangeduid kon worden. Een andere interpretatiewijze wordt momenteel onderzocht; dit betreft de verandering van de parameters van $25 \%$ of meer op twee opeenvolgende momenten. Deze wijze van interpretatie is een geaccepteerde wijze van andere (serum) tumormarkers. Daarnaast hebben we de toepasbaarheid van de macrofagentest in andere klinische situaties onderzocht. Het monitoren van radiochemotherapie of ziekteprogressie gedurende de follow-up periode na chirurgie zijn hier voorbeelden van. De mogelijkheid tot monitoring van radiochemotherapie bij rectumcarcinoom patiënten is onderzocht (paragraaf 2.4 en hoofdstuk 6). Momenteel wordt de monitoring van ziekteprogressie na afloop van chirurgie nog uitgevoerd. 


\subsection{Monitoring van het effect van radiochemotherapie bij rectumcarcinoom}

Een van de andere mogelijke applicaties werd gezien in de monitoring van de effecten (response predictie) van voorbehandeling middels radiochemotherapie (neoadjuvante therapie of $\mathrm{nCRT}$ ). Bij patiënten met rectum carcinoom is het geven van $\mathrm{nCRT}$ een gebruikelijke wijze van voorbehandelen. $\mathrm{nCRT}$ is gericht op het reduceren van de grootte van de tumor en het destrueren van lymfeklier metastasen. nCRT kan in potentie leiden tot verkleinen van de voorgenomen chirurgische resectie-gebied, en kan zelfs leiden tot een afwacht-en-monitor beleid indien de tumor als gevolg van de nCRT verdwijnt. Dit betekent dat een patiënt voordeel kan hebben van het voorspellen van de reactie op de nCRT omdat het mogelijk effect kan hebben op de daaropvolgende behandeling. Indien de voorspelling laat zien dat de kans groot is dat de patiënt in het geheel niet zal reageren op de reguliere $\mathrm{nCRT}$, er dan gekozen kan worden voor een alternatieve voorbehandeling, of zelfs voor direct uitvoeren van een chirurgische behandeling. $\mathrm{nCRT}$ bestaat uit een combinatie van bestraling (radiotherapie) en chemotherapie. Een gedachtegang was dat er door de tumor in het rectum tumorgerelateerd materiaal losgelaten wordt gedurende de periode van nCRT, en dat dit materiaal opgeruimd kan worden door macrofagen. Om de eventuele additionele waarde van macrofagen in het voorspellen van het effect van nCRT te bepalen, werd een groep rectumcarcinoom patiënten voorafgaand, gedurende en na afloop van nCRT onderzocht. Bloedanalyse bij rectum carcinoom patiënten gedurende de nCRT-periode werd uitgevoerd en PET-CT-scans werden gelijktijdig gemaakt.

Het bleek mogelijk om veranderingen te meten in meerdere parameters gedurende $\mathrm{nCRT}$, en sommigen van hen hadden voorspellende waarde. Het toevoegen van alle metingen aan reeds bestaande respons predictie modellen bleek te leiden tot een toename van de predictieve capaciteit van $72 \%$ naar $94 \%$ accuraatheid in de eerste twee weken van de nCRT. Dit betekent dat vroeg in de nCRT-periode het behandelteam met $94 \%$ zekerheid kan voorspellen of de gestarte voorbehandeling het beoogde effect zal hebben door het toevoegen van de nieuwe markers.

\subsection{Conclusie}

Concluderend, in dit promotietraject is gefocust op het onderzoeken van het principe van het gebruik van macrofagen in diagnostische setting in humaan 
weefsel en bloed, de ontwikkeling van een macrofagentest en de vroege toepassing ervan gedurende het monitoren van nCRT bij rectumcarcinoom patiënten. De test heeft de potentie om de predictieve waarde van predictiemodellen te verhogen en wordt momenteel onderzocht op additionele waarde in de follow-up periode na colorectale chirurgie. 



\section{Relevance}

Cancer is death cause number one in developed countries. Worldwide 12.4 million new cases of cancer occurred in 2008 , of which more than $50 \%$ is diagnosed in the developing countries ${ }^{1}$. In the US alone an estimate of 1.7 million new cancer patients is predicted for $2014^{2}$. The incidence in the Netherlands was 100.000 in 2011, and the incidence increased with 1.1 percent for men and 1.7 percent for women in the period from 2001 to 2011 . The total costs generated by cancer care in the Netherlands in 2011 was 4.8 billion Euro, of which almost $73 \%$ accounts for hospital care ${ }^{3}$. Besides the patient and family burden, the costs of cancer diagnostics and cancer treatment, also the economic impact of cancer is substantial. The worldwide economic impact of premature death and disability caused by cancer was 895 billion dollars in 2008. This analysis was made without taking the actual medical costs into account. Effectively this is 1.5 percent of the world's gross domestic product (GDP). The three cancer types causing the most economic impact globally per year were lung cancer, colorectal cancer and breast cancer, with the amounts of 188, 99 and 88 billion dollars respectively. Nations with smaller economies are less capable to allocate the needed financial funds to cancer diagnostic and treatment programs ${ }^{1}$.

\subsection{Diagnostic relevance}

All above mentioned facts underline the need for reliable cancer diagnostics, preferably low cost types. Reliable cancer diagnostics, in all settings, can aid in early cancer detection, in primary as well as secondary setting. Earlier detection could enable less invasive treatment options in both primary and secondary disease. Hereby improvement of quality of life for patients during and after the initial cancer detection phase is possible. This results in lower impact on patient, family and the global economy. Cancer treatment can only become more patient friendly and cost-efficient if cancer diagnostics are optimised first. Therefore population based screening has become an option for certain forms of cancer. Population based screening is known to reduce mortality for breast cancer, colorectal cancer, cervix cancer and lung cancer ${ }^{2}$. In the Netherlands the first results of a population-based screening-trial for colorectal cancer (CRC) have been reported with promising results. In 2014 almost 130.000 persons over 50 years of age were asked to perform an iFOBT test at home and over 15.000 persons tested positive. A colonoscopy was performed in over 10.000 persons

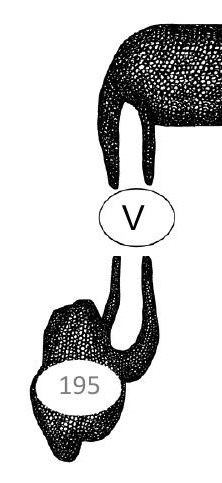


up until now. In 40 percent of this population CRC or advanced colorectal adenoma (AAD) was diagnosed. These results illustrate possible benefits of a population based screening for CRC. Risks of false positive results and complications in iFOBT and colonoscopy screening respectively are under investigation in this trial as well. The false positive rate (no CRC or AAD) of iFOBT in these first results was 58.9 percent as was confirmed by colonoscopy. In 31.9 percent of the persons with a positive iFOBT test not even low grade adenomas were found during colonoscopy. Colonoscopy has risks as well, such as bleeding and perforation ( 1.34 percent and $0.07 \%$ respectively). These problems are serious adverse events in an asymptomatic screening population ${ }^{4}$. If parts of the diagnostic trajectory could be optimised further, for example by exchanging one of the screening tools, a more reliable or less invasive screening protocol could be applied. This thesis elaborates on the investigation of epitope detection in macrophages (EDIM) as a candidate for screening purposes. The pilot study (chapter 4) in a randomly selected CRC-patient population showed a sensitivity and specificity of 100.0 percent and 83.3 percent respectively, using a cut-off point of 4.25 percent of CEA- in macrophages (CEAIM). In the validation study (chapter 5) it was shown the biological variability within a person leads to a range of normalcy values, which precludes singlesample analyses per person. Multiple analyses in a healthy person are needed to establish a range of normal values for this person. Aberrant values from that range would indicate presence of disease.

\subsection{Follow-up relevance}

In postoperative surveillance after colorectal surgery the carcinoembryonic antigen (CEA) serum test is combined with periodic ultrasound or CT-scan and colonoscopy. This surveillance protocol (including possible variations) have been captured in worldwide CRC follow-up guidelines ${ }^{5,6}$. The addition of the EDIM-method to the follow-up setting is currently under investigation. If an additional value could be found and it would outperform the diagnostic capacity of currently implemented follow-up strategies, then a possible change in the established protocols could become warranted. Additional costs would not outweigh the ability to detect recurrence earlier, to treat patients earlier with less invasive techniques. 


\subsection{Monitoring relevance}

Monitoring response to neoadjuvant treatment in rectal cancer patients could enable identification of responders and non-responders before or early during neoadjuvant therapy ${ }^{7}$. In combination with blood biomarkers this additional value was reported in a prediction model utilised before neoadjuvant treatment ${ }^{8}$. In our studies it was shown that the added value could be increased even further (AUC 0.94 ), by adding monocyte and macrophage analyses in combination with other serum markers and PET-CT values to a prediction model during the first two weeks of neoadjuvant treatment. When evaluating these outcomes upfront in all rectal cancer patients, the patients benefitting from neoadjuvant treatment could be identified. The patients who would not benefit could be treated according to other protocols or specific patients could refrain from therapy at all. These emerging options would lead to personalised medicine, that could lead to better quality of life for rectal cancer patients, and more appropriate spending of resources.

\section{Target groups, activities and products}

The medical specialities who could utilise the outcomes of this thesis arethe gastroenterologists and surgeons treating CRC patients. Immunologists could utilise the outcomes to further investigate the role of macrophages and monocytes in immunocompetence in cancer in general and specifically in CRC. When proven useful, development of an immuno-competence assay for cancer patients could be the next step. Oncologists could incorporate immunological assays (such as the EDIM-assay) in cancer follow-up settings for disease monitoring purposes. Finally, Radiotherapists could first investigate and at a later time point include monocyte and macrophage measurements, utilising the EDIM method, in treatment monitoring settings. National and global funding organisations should recognise the potential of immunological cancer research and expand their funding capacity towards this part of medical research. Participation of other (UM-) parties in further development of this assay could accelerate the development and broaden the applicability in medicine.

\section{Innovation}

The EDIM-method is a completely new way of utilising cells of the immune system, such as monocytes and macrophages, for diagnostic, follow-up and 
monitoring purposes. In prostate cancer this method was first described for diagnostic purposes by Herwig et al. ${ }^{9-12}$ and confirmed by Leers et al. in $2008^{13}$. The basic question of the actual localisation of tumour markers in macrophages was investigated by our group using the colorectal cancer model. Utilising light and electron microscopy the localisation of the antibody against CEA inside the monocytes and macrophages was visualised and confirmed ${ }^{14,15}$. In a pilot setting this innovative method was applied in a CRC diagnostic setting showing distinguishing capabilities between healthy and CRC patients. Addition of CRP allowed distinguishing $\mathrm{CRC}$ patients from benign inflammatory diseases of the colon $^{16}$. Validation of such an assay is innovative. All parts of the validation process must be conceived, tested and applied. This process has been performed and reported by our group ${ }^{17}$. The potential of applying the assay in CRC follow-up (research in progress) and in monitoring rectal cancer (paper submitted) has been and is currently being explored by our research group. The value of the EDIM-method in comparison to established diagnostic tools and other experimental methods in cancer diagnostics needs further evaluation.

\section{Planning and realisation}

Studies as described above are currently performed or have been performed with the results being published soon. 


\section{References}

1. American Cancer Society. The global economic cost of cancer. acspc-026203.pdf. 2010;

2. Society AC. Cancer Facts \& Figures 2014. American Cancer Society Atlanta; 2014;

3. Hoeveel zorg gebruiken mensen met kanker en wat zijn de kosten? [ [Internet]]. Nationaal Kompas Volksgezondheid. [cited 2014]. Retrieved from: http://www.nationaalkompas.nl/gezondheid-en-ziekte/ziekten-enaandoeningen/kanker/hoeveel-zorg-gebruiken-mensen-met-kanker-en-kosten/

4. Penning C. Landelijke monitoring van het bevolkingsonderzoek darmkanker: resultaten eerste halfjaar 2014. 2014;.

5. Duffy MJ, Lamerz R, Haglund C, Nicolini A, Kalousová M, Holubec L, and Sturgeon C. Tumor markers in colorectal cancer, gastric cancer and gastrointestinal stromal cancers: European group on tumor markers 2014 guidelines update. Int J Cancer. 2013;.

6. Kievit J. Follow-up of patients with colorectal cancer: numbers needed to test and treat. Eur J Cancer. England; 2002;38(7):986-99.

7. van Stiphout RG, Lammering G, Buijsen J, Janssen MH, Gambacorta MA, Slagmolen P, Lambrecht M, Rubello D, Gava M, Giordano A, Postma EO, Haustermans K, Capirci C, Valentini $V$, and Lambin P. Development and external validation of a predictive model for pathological complete response of rectal cancer patients including sequential PET-CT imaging. Radiother Oncol. Ireland; 2011;98(1):126-33.

8. Buijsen J, van Stiphout RG, Menheere PP, Lammering G, and Lambin P. Blood biomarkers are helpful in the prediction of response to chemoradiation in rectal cancer: A prospective, hypothesis driven study on patients with locally advanced rectal cancer. Radiother Oncol. 2014;.

9. Herwig R, Djavan B, Kramer G, El-Taieb MA, Kuhhas F, Leers M, and Marberger M. [Prostate cancer screening with a new marker based on circulating blood macrophages?]. Urologe A. 2007;46(9):1066-70.

10. Herwig R. Measurement of intracellular versus extracellular prostate-specific antigen levels in peripheral macrophages: a new approach to noninvasive diagnosis of prostate cancer. Clin Prostate Cancer. 2004;Dec;3(3):184-8.

11. Herwig R, Mitteregger D, Djavan B, Kramer G, Margreiter M, Leers MP, Glodny B, Haider DG, Hörl WH, and Marberger M. Detecting prostate cancer by intracellular macrophage prostatespecific antigen (PSA): a more specific and sensitive marker than conventional serum total PSA. European journal of clinical investigation. Wiley Online Library; 2008;38(6):430-437.

12. Herwig R, Horninger W, Rehder $P$, Klocker H, Ramoner R, Thurnher M, Pinggera GM, Gozzi C, Konwalinka G, and Bartsch G. Ability of PSA-positive circulating macrophages to detect prostate cancer. The Prostate. Wiley Online Library; 2005;62(3):290-298.

13. Leers MPG, Nap M, Herwig R, Delaere K, and Nauwelaers F. Circulating PSA-Containing Macrophages as a Possible Target for the Detection of Prostate Cancer. American journal of clinical pathology. American Society for Clinical Pathology; 2008;129(4):649.

14. Japink D, von Meyenfeldt MF, Nap M, Sosef MN, Verheyen F, Beets GL, and Leers MPG. Visualizing CEA in activated macrophages using electron microscopy. (submitted). 2014;.

15. Faber TJ, Japink D, Leers MP, Sosef MN, von Meyenfeldt MF, and Nap M. Activated macrophages containing tumor marker in colon carcinoma: immunohistochemical proof of a concept. Tumour Biol. Netherlands; 2011;33(2)(2):435-41.

16. Japink D, Leers MPG, Sosef MN, and Nap M. CEA in activated macrophages. New diagnostic possibilities for tumor markers in early colorectal cancer. Anticancer research. International Institute of Anticancer Research; 2009;29(8):3245.

17. Japink D, Nap M, Sosef MN, Nelemans PJ, Coy JF, Beets G, von Meyenfeldt MF, and Leers MP. Reproducibility studies for experimental epitope detection in macrophages (EDIM). J Immunol Methods. Netherlands; 2014;407:40-7. 



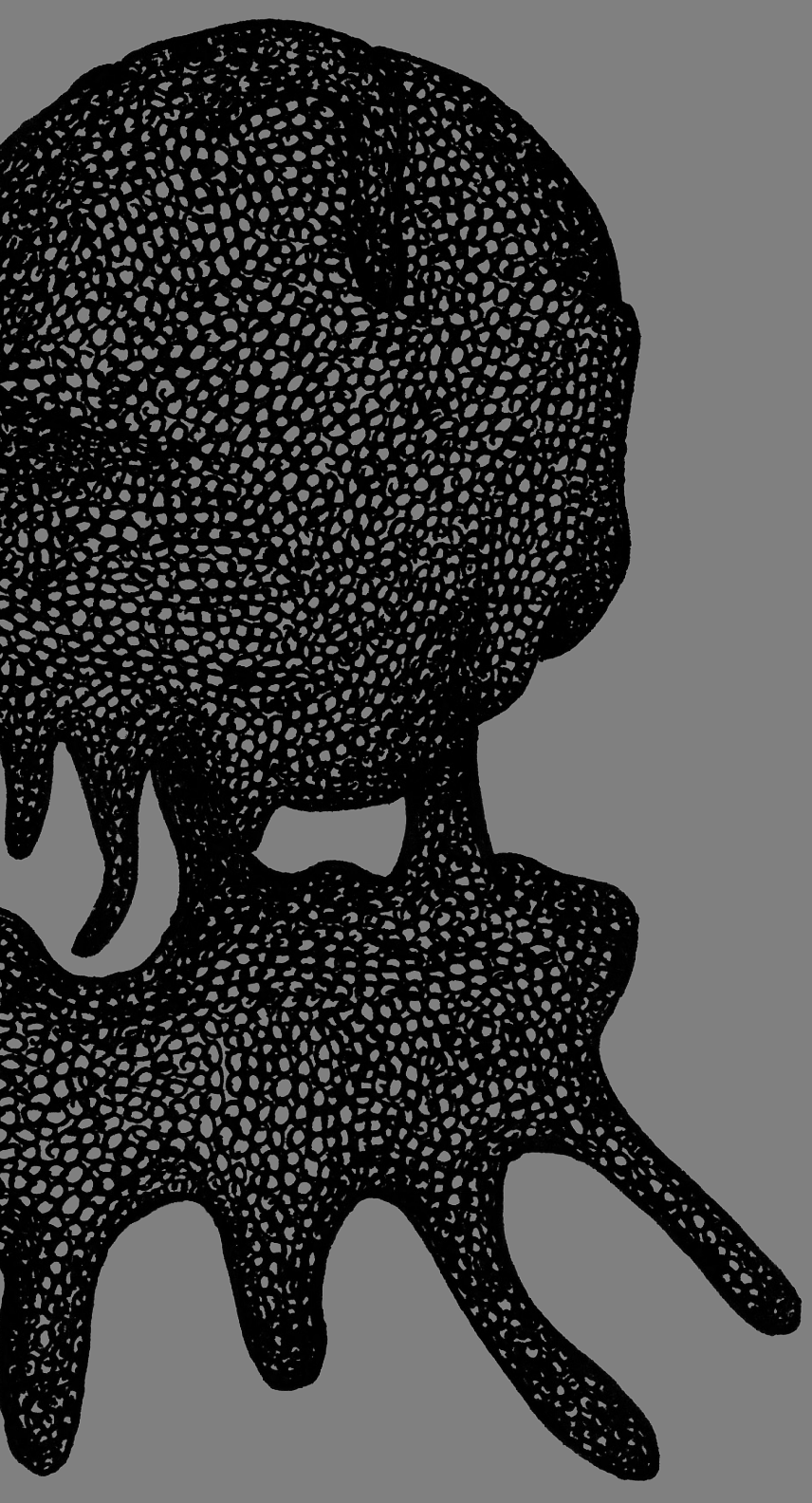

Dankwoord 


\section{Dankwoord}

Allereerst Maarten, mijn promotor. We waren al wat jaren onderweg toen we vanuit Heerlen bij je aanklopten om als promotor te fungeren. Het op de rijdende trein springen heb je als de beste gedaan, dank daarvoor. Wat hebben we in de afgelopen jaren veel aan tafel gezeten samen, eerst echt als promotor en promovendus, maar in de afgelopen jaren is het overleg voor mij echt gegroeid tot een vriendschappelijk overleg, wat ik zeer waardeer en waar we ook de wereld, het ziekenhuis en ons leven bespreken. Je wijsheid en "alles al gezien" hebbende heeft me meermaals behoedt voor enthousiaste acties die op niets zouden uitlopen, zowel in positieve als negatieve zin. Je hebt me naast veel input ook geleerd beter te schrijven, al hadden we allebei een andere methode, we kwamen samen uiteindelijk tot een pracht manuscript. Maarten bedankt en ik hoop dat we nog vaak samen mogen bomen.

Dan de copromotoren, ik heb er drie en daar ben ik trots op.

Laat ik beginnen met Marius, onze vriendelijke bebaarde patholoog in het team. Inspirerend als je bent op onderzoeks- en leidinggevend gebied, zo heb ik ook met je gediscussieerd, ben ik het met je eens geweest, maar ook even zo vaak oneens. Je onorthodoxe wijze van onderzoek doen, bespreken en schrijven hebben me wel eens op de kast gejaagd, maar daar hielp je me dan ook vlot weer vanaf. Je liet me door je enthousiasmering dingen uitzoeken, berekenen en in grafieken zetten waarvan ik eerst dacht dat ik dat niet voor elkaar zou krijgen, maar iedere keer lukte het toch weer. Ik ben er trots op dat je mijn copromotor bent, bowlen kun je toch echt niet en graag zie ik je nog eens zingen op congres (ja, dat filmpje heb ik nog)!

Dan hebben we Mathie, de flowcytometer-koning, stoere politieagent en natuurlijk dé klinisch chemicus en hematoloog in ons team. Je bent onmisbaar geweest en onze gesprekken bij kopjes koffie, soms wekelijks, soms wat intermitterender zijn me veel waard geweest. Je bent een binnenvetter en dat je daarin soms onleesbaar bent heeft me nog wel eens hoofdbrekens opgeleverd, en zo nu en dan een onder-vier-ogen gesprekje, maar ook daar zijn we goed uitgekomen. Je hebt me kritisch leren kijken naar flowcytometrie-werkwijzen en interpretaties, wat me geholpen heeft om alle metingen goed te verwerken. Je stimulerende woorden en het feit dat ik altijd bij je kon komen met een vraag (of veel vragen) hebben me door deze jaren heen gesleept. Als copromotor kon ik me geen betere wensen, dankjewel! 
Meindert tot slot, mijn chirurgie-maatje, wat onwennig begonnen toen je zelf net in Heerlen was, maar al vlot een bruisend vat van energie die het woord stimulus zelf uitgevonden kon hebben. Ik heb me in het onderzoek in het begin altijd afgevraagd of je überhaupt wel snapte wat ik aan het doen was, maar gedurende overleggen met de anderen kon je dat eigenlijk vrij eenvoudig uitleggen, je snapte het technische deel echt niet. Het klinische deel daarentegen was jouw pakkie an en nu nog ga je er helemaal in op. Ik ben weet ik niet hoe vaak bij je langs geweest om te overleggen, advies te vragen en (ja ook hier) een bakkie te doen. Stimulerend ten top, altijd snel met reageren, ja ook soms irrelevante antwoorden, maar ach. Het is inderdaad zo dat wellicht de beste stimulans je woorden van vorig jaar waren, "dat het me in 2014 niet ging lukken om alles af te krijgen". Daarin heb je in ieder geval ongelijk gehad, een prettige conclusie om te trekken. In je taak in dit traject was en ben je een chirurgisch copromotor van formaat, dank!!

Dear thesis judging committee; thank you for taking the time to read all my work, judge it properly and provide me with proper feedback in time. I really appreciate the time and effort you all put in. Hope to see you all during the defence and in future research or other circumstances.

Dan nog de overige begeleiders van het promotietraject:

Lieve Patty, onze klinisch epidemiologe bij het team. Als ik een ding geleerd heb van je dan is het de onderzoeksvraag helder te hebben. Een tweede ding wat ik van je geleerd heb is dat je dat dan ook moet doen voorafgaand aan je dataverzameling. In beide zijn we regelmatig niet geslaagd en dan kreeg ik weer een zuchtende en steunende Patty voor mijn neus, werd ik weer even stevig in de hoek gezet en konden we daarna constructief aan de slag met de gegevens die dan toch wel weer op een of andere manier grondig geanalyseerd konden worden. Je kennis is indrukwekkend en ik heb, ondanks je af en toe broodnodig pittige reacties, altijd met veel plezier je kamer bezocht. Dank daarvoor.

Beste Geerard, Prof Beets onderhand, analytisch, kritisch op methode en inhoud en toch ook wel een beetje van je stuk te krijgen als ik "out of the box" roep dat de huidige follow-up van het rectumcarcinoom toch zo maar eens op de schop zou kunnen gaan. Immunologie kan een rol spelen, jij ziet en zag dat toch wat genuanceerder. Maar dat komt nog wel... Nu we gezamenlijk in de kliniek staan is het ineens iets anders allemaal, maar ook nu komen we er samen altijd uit. Ik 
heb bewondering voor je voornoemde eigenschappen en hoopte de komende jaren nog veel chirurgisch van je op te steken, echter mag dat helaas niet zo zijn. Heel veel succes in je nieuwe positie noordelijker in het land.

Dan een groep collegae zonder wie dit proefschrift er al helemaal niet zou zijn, mijn lieftallige WESP-studenten in volgorde van meedraaien in het project: Roy, Philip (ook keuzestage), Noortje, Jeroen, Kim, Nina, Tjitske, Jogien, Martine en David (ook keuzestage). Gedurende meerdere jaren maakten jullie in wisselende samenstelling deel uit van het team en dit vonden we allen geweldig. $\mathrm{lk}$ vind het prachtig om te zien dat een deel van jullie ook in ons prachtige chirurgische vak terecht is gekomen naar aanleiding van jullie stage. Maar ook de rest is goed geland heb ik gezien. Wat een enorme lading buisjes hebben we met z'n allen weggewerkt, zeker meer dan 10.000 stuks... kun je het je nog herinneren, of heb je het stiekem ver weg gestopt... Jeroen en Tjits, jullie coauteurschappen staan als een huis, trots op jullie!

Dan nog een aantal afdelingen in het Atrium:

Allereerst mijn lab-collegae in het Atrium, iedereen was altijd zo vriendelijk en behulpzaam, we hadden het niet kunnen regelen zonder jullie. Met name de prikdienst in Heerlen, Kerkrade en Brunssum, maar ook de thuisprikdienst en alle laboranten in Heerlen. Met nadruk wil ik de trialanalisten noemen, maar ook de heren en de dames van de ICT op het lab en last but not least de lieve collegae op het beenmerglab, onder de bezielende leiding van Annie.

Ook het pathologie lab in Heerlen is onmisbaar geweest voor het slagen van dit traject. Alle medewerkers daar, waaronder Math, Jack, Ludy en de andere analisten. En natuurlijk Nick, onze flowcytometrie en Mathlab kenner, hopelijk ben je al vergevorderd met jouw proefschrift, we hebben lol gehad met het geven van de flow-cursus voor dummies.

Dan de afdeling chirurgie in Heerlen, Brunssum en Kerkrade.

Allereerst de dames van het secretariaat, de polidames en heer, de verpleging van afdeling 11 en 12 en de andere etages; in het bijzonder Leintje, de afdelingssecretaresses van 11 en 12 en tot slot de OK-medewerkers die altijd klaar stonden om bloed te samplen voor mijn studie. 
Niet te vergeten mijn steun en toeverlaat in de inclusies, de MDLverpleegkundigen Desiree, Ilse en Grietje, superbedankt! Verder, de dames en heren van de scopie kamer in Kerkrade, zonder jullie hadden we niet zo'n mooie controle groep gehad.

Dan de afdelingen in het MUMC

Allereerst de dames van het secretariaat Heelkunde, in het bijzonder Joyce, Sabeth en Evelien voor het regelen van alle zaken die we nodig hadden. Op de afdeling ook Marian en Mariëlle als kapiteins op het schip. Cees Dejong voor het geloven in mijn traject en het ondersteunen waar nodig.

Het Heelkunde lab alwaar ik Wim, Mo, Sander en Kaatje het meest meegemaakt heb, maar ook alle andere lab collegae hartelijk dank voor jullie kritische noten gedurende mijn presentaties en literatuurbesprekingen. Maar ook mijn wisselende roomies in het afgelopen schrijfjaar, dank voor jullie gezelligheid en steun!

Het klinisch chemisch lab, het was kort maar krachtig, dank voor jullie ondersteuning.

Afdeling Pathologie in het MUMC; Adriaan, toen je er nog werkte, dank voor je scherpe blik, Math Rousch dank voor je flow-sort cursus en je vertrouwen in mij dat ik met je flow-sort-kindje mocht spelen.

De afdeling Electronenmicroscopie, met name Fons en Hans, dank voor jullie input, tijd en inzet. Zonder jullie was dit boekje een hoofdstuk armer.

Dan MAASTRO-clinic, Guido Lammering, Jeroen Buijsen en Ruud van Stiphout, soms kwamen we er niet helemaal uit waar artikelen over moesten gaan, maar dat is uiteindelijk helemaal goed gekomen. Dank voor het mede faciliteren van het onderzoek. Dat geldt ook voor de dames van het bloedafname-lab van de MAASTRO, die alle patiënten hebben ingelicht en geprikt. Zeker net zo belangrijk zijn de dames van het datamanagement (Ann Claessens, Sylvia Loffeld en anderen), zonder hen hadden we niet alle data op een rijtje gehad. Allen superbedankt! 
Beckton \& Dickinson, met name Frans Nauwelaers wil ik bedanken voor het ondersteunen van de onderzoeken die we verricht hebben. Ook je kritische opmerkingen, je behulpzaamheid en je vriendelijke belse tongval hebben ons altijd voorzien van de juiste stimulans om weer verder te kijken en door te pakken. Het boekje was er niet geweest zonder jullie.

Johannes Coy en onze collegae onderzoekers van Tarvarlin en Tavargenix in Darmstadt. Ook de collegae laboranten Martina en Olaf van InVitaLab te Neuss. Allen dank voor de ondersteuning en het meedenken in de validatiestudies, wat een klus was dat. Veel succes met het vermarkten van het idee van de EDIMtest.

Dan honderdduizend maal dank voor wellicht de belangrijkste groep personen van het gehele traject: Onze patiënten, gezonde proefpersonen en patiënten met goedaardige darmaandoeningen zonder wie we het onderzoek nooit hadden kunnen doen. Hopelijk heeft dit proefschrift een aanzet geleverd voor toepassing van de gevonden resultaten in de kliniek en verder onderzoek, zodat de patiënten van de toekomst ervan kunnen profiteren.

Peter, we hebben samengewerkt op een totaal ander gebied, de ICT, en we waren een topteam. In die anderhalf jaar die tezamen liep met mijn promotie hebben we elkaar en elkaars familie goed leren kennen. Ik heb je wijze lessen goed in mijn oren geknoopt en zal de "s" koste wat het kost wegpoetsen. Heel veel succes en sterkte in je werk- en privéleven, je bent een kanjer.

Mijn schoonmoeder Marjo en schoonzussen An en len voor het tonen van begrip dat ik wel eens niet meekwam omdat ik geen tijd had. Dat is natuurlijk niet omdat ik jullie niet lief vind, maar juist niet wilde vermoeien met verhalen over dit traject en de totstandkoming van dit boekje. De momenten dat ik er wel was kon ik dit traject gelukkig makkelijk even parkeren door de gezellige positieve drukte die er altijd heerst als we samen zijn. Dank dat ik erbij hoor!

Mijn zus, zwager en kinderen die ik de afgelopen jaren spaarzaam heb bezocht en heb kunnen bezoeken. Laten we hier in de komende jaren samen aan werken! 
Mijn lieve ouders, die al zo vaak zeggen dat ze het fantastisch vinden wat ik gedaan heb en nu doe, ik voel me vereerd dat ik jullie zoon ben, heb veel van jullie geleerd. Over hoe in het leven te staan en wat je moet doen om iets te bereiken in het leven, nooit opgeven is het devies. In de afgelopen jaren hebben jullie me door dik en dun gesteund, waardoor het volbrengen van dit lijdenswegje me toch lichter is gevallen. Als ik er niet altijd over praatte was het meer dat ik dan blij was dat ik het even kon parkeren, en over andere zaken kon babbelen. Afschakelen is iets wat ik bij jullie prima kan, dank daarvoor. Hopelijk zien we elkaar nog lang wekelijks als jullie op ons lieve dochtertje passen. Ben heel blij met jullie!

Sienna, mijn dochter, voor het lieve wondertje dat je bent ben ik jou maar vooral ook je mama dankbaar, maar in de korte periode dat je er bent, ben ik helemaal verknocht aan je geraakt. Papa's kindje zeggen ze, en dat is ook zo, je bent het zonnetje wat al schijnt voor zonsopgang en je straalt totdat de echte zon allang is onder gegaan. Jouw papa zijn is een feest. Ben gek op je...

Joëlle... sprakeloos ben ik door je, keer op keer, de mooiste vrouw van allemaal! Altijd lief en vergevingsgezind, ook als ik toch weer achter de computer moest kruipen. Als (saaie, NOT!) internipst met passie voor niertjes ben je niet alleen de mooiste internipst, maar ook nog eens een hele slimme. Zeker meer dan een wandelend kruidenalmanakje, nu ben je zeker al de luxe editie van de grote Winkler Prins, op weg naar je eigen ultieme versie. Dat gaat je lukken. Je creëerde mijn ideale werkomgeving, maar als je vond dat het te lang duurde kwam je me gewoon halen voor een stukje ontspanning. Nu kunnen we zonder dit soort obstakels verder op weg naar onze toekomst samen (dat ja-woord is onderweg, dat weet je). Eerst gaf je me al je hart en afgelopen februari gaf je me onze dochter, wat een wereldervaring zo met z'n tweeën. Lieverd, ik hou van je.... 


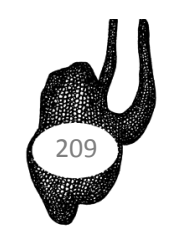





\section{Curriculum Vitae}




\section{Curriculum Vitae}

Dennis Japink werd geboren op 20 september 1978 in Ermelo. Na het HAVOdiploma in 1995 haalde hij het Atheneum-diploma in 1997. Hij werd uitgeloot voor de studie geneeskunde en besloot daarom farmacie te gaan studeren in Groningen. Na nog 3 maal uitgeloot te zijn werd hij alsnog nageplaatst voor de studie Geneeskunde te Maastricht. Gedurende zijn studie verbleef hij tweemaal in het buitenland; allereerst een keuzestage Klinisch Immunologie in Linköping in Zweden alwaar zijn interesse voor het menselijk immuunsysteem werd gewekt en vervolgens nog een co-schap KNO in Pretoria in Zuid-Afrika. In het laatste jaar van zijn geneeskundeopleiding (2006-2007) heeft hij gedurende zijn gecombineerde klinische en wetenschapsstage de basis gelegd voor dit promotieonderzoek door een prospectieve studie op te zetten in het Atrium Medisch Centrum Parkstad, te Heerlen. Hij behaalde vervolgens in 2007 het artsexamen aan de Universiteit Maastricht. Voor zijn onderzoekswerk in het laatste jaar van de geneeskundestudie ontving hij de NVGE Student Award in 2007. Na afloop van zijn studie werd hij ANIOS Heelkunde in het Atrium Medisch Centrum Parkstad, te Heerlen. Begin 2008 werd hij full-time onderzoeker, eerst alleen in Heerlen, later ook in het Maastricht University Medical Center. Het onderzoek vond plaats onder leiding van de co-promotores dr. Sosef (gastrointestinaal en oncologisch chirurg), dr. Nap (klinisch patholoog) en dr. Leers (klinisch chemicus en hematoloog), met als promotor Prof. dr. von Meyenfeldt (oncologisch chirurg). In 2009 volgde zijn eerste publicatie over het onderwerp van dit proefschrift en ontving hij de MAASTRO-Atrium grant. In 2010 verwierf hij een AGIKO-subsidie van ZonMw. Begin 2011 startte hij met de gecombineerde opleiding tot chirurg en klinisch onderzoeker, met één jaar volledig onderzoek. In de jaren daaropvolgend publiceerde hij verschillende papers over het in dit proefschrift beschreven werk. In de periode 2012-2013 rondde hij de eerste twee jaar van zijn chirurgische opleiding af, gevolgd door wederom een volledig jaar wetenschapsbeoefening in 2014. Per 1-1-2015 is hij begonnen aan zijn 2 e periode van 2 jaar chirurgische opleiding, ditmaal in het Maastricht University Medical Center te Maastricht. Hij zal zijn opleiding tot chirurg afronden in het Atrium Medisch Centrum Parkstad te Heerlen aansluitend op de huidige periode in Maastricht. 



\section{List of publications}

Japink D, Leers MP, Sosef MN, Nap M. CEA in activated macrophages. New diagnostic possibilities for tumor markers in early colorectal cancer. Anticancer Res. 2009;29:3245-51.

Telgenkamp B, Japink D, van Haaren E. Cardiac Arrest after Patent Blue V Injection for Sentinel Lymph Node Biopsy in Breast Cancer. Breast Care (Basel). 2010;5:411-413

Schouten R, Japink D, Meesters B, Nelemans PJ, Greve JW. Systematic literature review of reoperations after gastric banding: is a stepwise approach justified? Surg Obes Relat Dis. 2011;7:99-109.

Faber TJ, Japink D, Leers MP, Sosef MN, von Meyenfeldt MF, Nap M. Activated macrophages containing tumor marker in colon carcinoma: immunohistochemical proof of a concept. Tumour Biol. 2012;33:435-41.

Japink D, Nap M, Sosef MN, Nelemans PJ, Coy JF, Beets G, von Meyenfeldt MF, Leers MP. Reproducibility studies for experimental epitope detection in macrophages (EDIM). J Immunol Methods. 2014;407:40-7.

Japink D, von Meyenfeldt MF, Nap M, Sosef MN, Beets GL, Leers MPG. Macrophages in colorectal cancer: current diagnostic opportunities. European Journal of Cancer 2015. Ingediend.

Japink D, Bransen J, Buijsen J, van Stiphout RGPM, Nap M, Leers MPG, Nelemans PJ, Beets GL, von Meyenfeldt MF, Sosef MN. Predicting response using blood biomarkers and PET-CT during neoadjuvant chemoradiation in rectal cancer patients, Annals of Surgery 2015. Ingediend.

Japink D, von Meyenfeldt MF, Nap M, Sosef MN, Verheyen F, Beets GL, Leers MPG. Visualising CEA in activated macrophages using electron microscopy, PlosOne 2015. Ingediend. 



\section{Abbreviations}

\begin{tabular}{|c|c|}
\hline AAD & Advanced adenomas \\
\hline AEC & 3-amino-9-ethylcarbazole \\
\hline AJCC & American joint committee on cancer \\
\hline AM & Activated macrophages \\
\hline APC & Allophycocyanin \\
\hline APC & Adenomatous polyposis coli \\
\hline AUC & Area under the curve \\
\hline $\mathrm{BC}$ & Blood capillairies \\
\hline bFGF & Basic fibroblast growth factor \\
\hline BGP & Biliary glycoproteins \\
\hline BRAF & Proto-oncogene B-Raf, serine/threonine kinase \\
\hline BSA & Bovine serum albumin \\
\hline CBC & Complete blood count \\
\hline CCR2 & Chemokine receptor 2 \\
\hline CD & Crohn's disease \\
\hline CD in CD14 or CD16 & Cluster of differentiation \\
\hline CEA & Carcinoembryonic antigen \\
\hline CEA-IM & CEA in macrophages (CEACM) \\
\hline CEA-S & Isomeric carcinoembryonic antigen \\
\hline CEACM & CEA-containing macrophages (CEA-IM) \\
\hline CGM & CEA gene family members \\
\hline CK & Cytokeratin \\
\hline CK-IM & CK in macrophages \\
\hline CMP & Common myeloid progenitor \\
\hline CRC & Colorectal Cancer \\
\hline CRP & C-reactive protein \\
\hline CSF-1 & Colony stimulating factor 1 \\
\hline CTC & Circulating tumour cells \\
\hline ctDNA & Circulating tumour DNA \\
\hline $\mathrm{DAB}$ & 3,3'-Diaminobenzidine \\
\hline DC & Dendritic cells \\
\hline DFS & Disease free survival \\
\hline dMMR & Defective mismatch repair \\
\hline EDIM & Epitope Detection In Macrophages \\
\hline EDTA & Ethylenediaminetetraacetic acid \\
\hline EGF & Endothelial growth factor \\
\hline EGFR & Endothelial growth factor receptor \\
\hline EM & Electron microscopy \\
\hline
\end{tabular}




\begin{tabular}{|c|c|}
\hline ERUS & Endorectal ultrasound \\
\hline FAP & Familial adenomateus polyposis \\
\hline Fcgamma & (FC: Fragment, crystallizable) region directed at $\lg G$ \\
\hline $\mathrm{FI}$ & Fluorescence intensity \\
\hline FIT & Faecal immunochemical test \\
\hline FITC & Fluorescein isothiocyanate \\
\hline FMO & Fluorescence-minus-one \\
\hline FSC & Forward Scatter \\
\hline GA & Glutaraldehyde \\
\hline GDP & Gross domestic product \\
\hline gFOBT & Guaca faecal occult blood test \\
\hline GFP & Green fluorescin protein \\
\hline$H \& E$ & Hematoxylin \& eosin staining (HE) \\
\hline HNPCC & Hereditary non-polyposis colorectal cancer \\
\hline HSC & Haematopoietic stem cells \\
\hline IBD & Inflammatory bowel disease \\
\hline ICC & Intra-class correlation coefficient \\
\hline IEM & Immuno electron microscopy \\
\hline iFOBT & Immunochemical feacal occult blood test \\
\hline IL10 & Interleukin 10 \\
\hline IL12 & Interleukin 12 \\
\hline IL8 & Interleukin 8 \\
\hline INF & Immune cell influx \\
\hline KC & Kupffer cells \\
\hline KRAS & Kirsten rat sarcoma viral oncogene homolog (K-ras) \\
\hline LARC & Locally advanced rectal cancer \\
\hline LM & Light microscopy \\
\hline LPS & Lipopolysaccharide \\
\hline M30 & M30-neo-epitope \\
\hline M30-IM & M30 in macrophages \\
\hline MAP & MYH-associated polyposis \\
\hline MC & Methylcellulose \\
\hline MdnFI & Median fluorescence intensity \\
\hline $\mathrm{mFMO}$ & Modified Fluorescence-minus-one \\
\hline MLH1 & $\begin{array}{l}\text { MutL homolog } 1 \text {, colon cancer, nonpolyposis type } 2 \\
\text { (Lynch syndrome mutation) }\end{array}$ \\
\hline MMP & Matrix metallo proteinase \\
\hline MREC & Medical research ethics committee \\
\hline MSH2 & MutS protein homolog 2 (Lynch syndrome mutation) \\
\hline MTA & Membrane-associated tissular antigen \\
\hline
\end{tabular}




$\begin{array}{ll}\text { MTV } & \text { Metabolic tumour volume } \\ \text { NCA } & \text { Non-specific crossreacting antigens } \\ \text { nCRT } & \text { Neoadjuvant chemoradiation therapy } \\ \text { nm } & \text { Nanometer } \\ \text { OR } & \text { Operating room } \\ \text { PB } & \text { Phosphate buffer } \\ \text { pCR } & \text { Pathological complete response } \\ \text { PE } & \text { Phycoerythrin } \\ \text { PFA } & \text { Paraformaldehyde } \\ \text { PIK3CA } & \text { Phosphatidylinositol-4,5-bisphosphate 3-kinase, } \\ & \text { catalytic subunit alpha } \\ \text { PSA } & \text { Prostate specifice antigen } \\ \text { PSG } & \text { Pregnancy-specific } \beta \text {-1 glycoprotein } \\ \text { RI } & \text { Response index } \\ \text { ROC-curve } & \text { Receiver operating characteristic curve } \\ \text { RT } & \text { Room temperature } \\ \text { SD } & \text { Standard Deviation } \\ \text { SOP } & \text { Standard Operating Procedure } \\ \text { SSC } & \text { Side Scatter } \\ \text { SUV } & \text { Standard uptake value } \\ \text { TAM } & \text { Tumour associated macrophages } \\ \text { TEM } & \text { Transmission electron microscopy } \\ \text { TFAP2E } & \text { Transcription factor AP-2 epsilon } \\ \text { TGL } & \text { Tumour gland lumen } \\ \text { TIMP1 } & \text { Metallopeptidase inhibitor 1 } \\ \text { TKTL1 } & \text { Transketolase-like 1 } \\ \text { TKTL1-IM } & \text { TKTL1 in macrophages } \\ \text { TM } & \text { Tumormarkers } \\ \text { TRG } & \text { Tumour regression grade } \\ \text { UA } & \text { Uranylacetate } \\ \text { UC } & \text { Ulcerative colitis } \\ \text { ypTN } & \text { Tumour response } \\ & \end{array}$


